\title{
Measurement of the $B$ meson Lifetimes with the Collider Detector at Fermilab
}

\author{
Satoru UOZUMI \\ (Doctoral Program in Physics)
}

Submitted to the Graduate School of

Pure and Applied Sciences

in Partial Fulfillment of the Requirements

for the Degree of Doctor of Philosophy in Science

at the

University of Tsukuba 



\section{Abstract}

The lifetimes of the $B^{-}, \bar{B}^{0}$ and $\bar{B}_{s}^{0}$ mesons are measured using partially reconstructed semileptonic decays. Following semileptonic decay processes and their charge conjugates are used for this analysis:

$$
\begin{aligned}
B^{-} / \bar{B}^{0} & \rightarrow \ell^{-} \bar{\nu} D^{0} X \\
B^{-} / \bar{B}^{0} & \rightarrow \ell^{-} \bar{\nu} D^{*+} X \\
\bar{B}_{s}^{0} & \rightarrow \ell^{-} \bar{\nu} D_{s}^{+} X
\end{aligned}
$$

where $\ell^{-}$denotes either a muon or electron.

The data are collected during 2002 - 2004 by the $8 \mathrm{GeV}$ single lepton triggers in CDF Run II at the Fermilab Tevatron Collider. Corresponding integrated luminosity is about 260 and $360 \mathrm{pb}^{-1}$ used for the $B^{-} / \bar{B}^{0}$ and $\bar{B}_{s}^{0}$ lifetime analyses, respectively. With the single lepton triggers, events which contain a muon or electron with a transverse momentum greater than $8 \mathrm{GeV} / c$ are selected. For these lepton candidates, further lepton identification cuts are applied to improve purity of the $B$ semileptonic decay signal.

After the lepton selection, three types of charm mesons associated with the lepton candidates are reconstructed. Following exclusive decay modes are used for the charm meson reconstruction:

$$
\begin{aligned}
& D^{0} \rightarrow K^{-} \pi^{+} \\
& D^{*+} \rightarrow D^{0} \pi_{s}^{+}, \text {followed by } D^{0} \rightarrow K^{-} \pi^{+} \\
& D_{s}^{+} \rightarrow \phi \pi^{+}, \text {followed by } \phi \rightarrow K^{+} K^{-} .
\end{aligned}
$$

Here $\pi_{s}^{+}$denotes a slow pion from $D^{*+}$ decay. Species of the reconstructed charm meson identify the parent $B$ meson species. However in the $B^{-} / \bar{B}^{0}$ semileptonic decays, both mesons decay into the identical lepton $+D^{0}$ final state. To solve this mixture of the $B$ components in the $D^{0}$ sample, we adopt the following method: First among the inclusive $D^{0}$ sample, we look for the $D^{*+} \rightarrow D^{0} \pi_{s}^{+}$signal. The inclusive $D^{0}$ sample is then split into the two samples of $D^{0}$ mesons which are from the $D^{*+}$ meson and not from $D^{*+}$. We use the fact that $D^{*+}$ sample is dominated by the $\bar{B}^{0}$ component, and the $D^{0}$ sample after excluding the $D^{*+}$ events is dominated by the $B^{-}$component. Fraction of remaining mixture of $B^{-} / \bar{B}^{0}$ components in each sample is estimated using a Monte Carlo simulation.

From the lepton + charm meson pairs, we measure the $B$ meson decay lengths to extract the lifetimes. Since the $B$ meson momentum, necessary to calculate the $B$ meson decay time, is not fully reconstructed in semileptonic 
decays, the missing momentum is corrected using a Monte Carlo simulation during lifetime fits. Also, contributions of various kinds of backgrounds are considered and subtracted.

As a result of the fit, the $B$ meson lifetimes are measured to be

$$
\begin{aligned}
c \tau\left(B^{-}\right) & =495.6 \pm 8.6_{-12.8}^{+13.3} \mu \mathrm{m} \\
c \tau\left(\bar{B}^{0}\right) & =441.5 \pm 10.9 \pm 17.0 \mu \mathrm{m} \\
c \tau\left(\bar{B}_{s}^{0}\right) & =414.0 \pm 16.6_{-13.8}^{+15.6} \mu \mathrm{m}
\end{aligned}
$$

or

$$
\begin{aligned}
\tau\left(B^{-}\right) & =1.653 \pm 0.029{ }_{-0.031}^{+0.033} \mathrm{ps} \\
\tau\left(\bar{B}^{0}\right) & =1.473 \pm 0.036 \pm 0.054 \mathrm{ps} \\
\tau\left(\bar{B}_{s}^{0}\right) & =1.381 \pm 0.055_{-0.046}^{+0.052} \mathrm{ps}
\end{aligned}
$$

and the lifetime ratios to be

$$
\begin{aligned}
\tau\left(B^{-}\right) / \tau\left(\bar{B}^{0}\right) & =1.123 \pm 0.040_{-0.039}^{+0.041} \\
\tau\left(\bar{B}_{s}^{0}\right) / \tau\left(\bar{B}^{0}\right) & =0.938 \pm 0.044_{-0.046}^{+0.049}
\end{aligned}
$$

where the first uncertainty is statistical and the second is systematic. 


\section{Acknowledgments}

First of all, I would like to thank Prof. Shinhong Kim and Prof. Fumihiko Ukegawa for giving me really lots of great guidances and advices totally during 4 years of periods which I was working on the CDF experiment. They gave me an great chance to work at the world's highest collider experiment. And when I was sometimes sticked on some problems, they always gave me impressive advices and encouragements.

I thank the $B$ group people, Franco Bedeschi, Alessandro Cerri, David Clark, Sinead Farrington, Stefano Giagu, Guillelmo Gomez-Ceballos, Matt Herndon, Joe Kroll, Manfred Paulini, Christoph Paus, Kevin Pitts, Marjorie Shapiro, Masa Tanaka, Ilya Kravchenko, Tatjana Unverhau, Barry Wicklund who frequently gave me useful advices and opinions about the analysis.

I want to appreciate staffs of my home institution, Prof. Koji Takikawa, Dr. Kazuhiko Hara, Dr. Takasumi Maruyama, Dr. Yuji Takeuchi, Dr. Hiroyuki Matsunaga, Dr. Tomonobu Tomura, Prof. Yoshihiro Seiya, Mrs. Kazuko Kumashiro who are (were) belonging to high energy physics laboratory at University of Tsukuba.

I also thank sharing 2 years at Fermilab with me, Hirokazu Kobayashi, Takashi Ogawa, Tomohiro Yamashita, Takasumi Maruyama, Hideaki Takano, Naho Tanimoto, Soushi Tsuno, Koji Sato, Tatjana Unverhau, Isamu Nakamura, Nobu Oshima, Kyoko Kunori, Yoshiyuki Miyazaki, Koji Ikado, who we had a lot of good times and experiences. Especially, Hirokazu shares really long time at Fermilab with me, not only at the office but also at the house, and almost all of lunches and dinners. Tatjana sometimes gave me amusing e-mails when I am tired with the analysis. Nobu treated us delicious dinner and good time so often, about once a week.

I want to thank my friends no relate to the physics, but sharing great times on my hobby, mountaineering.

I really appreciate to my family and relatives, Yukio, Fumiko, Yukiko, Yuko, Shigeru and Chiyo Matsui, Hajime, Mariko, uncles and aunts, cousins, for giving me a plenty of spiritual helps and economic backups. I could not finish this work without their helps and understanding. My father Yukio supports me in all aspects for a long, long time of my student life. I believe that my mother Fumiko really would have loved to see this thesis. Matsui and Yamamoto families give lots of good times when I sometimes back to Kosai. They also gave me great spiritual supports and helps on every side, every time. My grandparents Shigeru and Chiyo are giving unfailing love since I was born. I have no words to express how grateful everything they gave me. 
I thank all the people who are (were) working on the CDF experiment, Fermilab staff and the technical staffs of the participating institutions for their vital contributions.

This work was supported by the U.S. Department of Energy and National Science Foundation; the Italian Istituto Nazionale di Fisica Nucleare; the Ministry of Education, Culture, Sports, Science and Technology of Japan; the Natural Sciences and Engineering Research Council of Canada; the National Science Council of the republic of China; the Swiss National Science Foundation; the A. P. Sloan Foundation; the Bundesministerium fuer Bilding und Forschung, Germany; the Korean Science and Engineering Foundation and the Korean Research Foundation; the Particle Physics and Astronomy Research Council and the Royal Society, UK; the Russian Foundation for Basic Research; the Comision Interministerial de Ciencia y Technologia, Spain; and in part by the European Community's Human Potential Programme under contract HPRN-CT-20002, Probe for New Physics. 


\section{Contents}

1 Introduction $\quad \mathbf{1}$

1.1 Weak interaction and CKM matrix .......... 2

1.2 Theoretical overview of the $B$ meson lifetimes . . . . . . . . 3

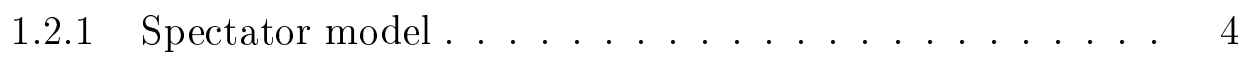

1.2 .2 Non-spectator effects . . . . . . . . . . . . . . . 5

1.2.3 Heavy Quark Expansion . . . . . . . . . . . 6

1.3 Motivation of the $B$ meson lifetime measurement $\ldots . . . .8$

1.4 Analysis outline . . . . . . . . . . . . . . 9

2 Experimental Apparatus 11

2.1 Accelerator complex . . . . . . . . . . . . . . 12

2.1.1 Proton source . . . . . . . . . . . . . 12

2.1.2 Anti-proton source . . . . . . . . . . . 13

2.1 .3 Tevatron ...................... 13

2.2 The Collider Detector at Fermilab . . . . . . . . . . . . 15

2.2.1 Coordinate system . . . . . . . . . . . 17

2.2 .2 Tracking detectors . . . . . . . . . . . 17

2.2.3 Solenoid magnet . . . . . . . . . . . . . 24

2.2 .4 Calorimeter ................... 24

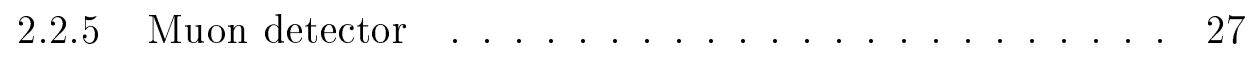

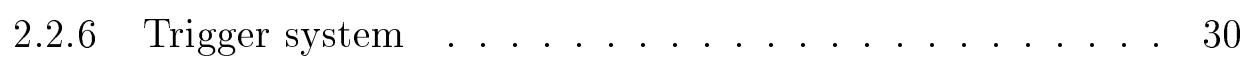

2.2.7 $8 \mathrm{GeV}$ single lepton triggers . . . . . . . . . . 32

3 Event reconstruction $\quad 35$

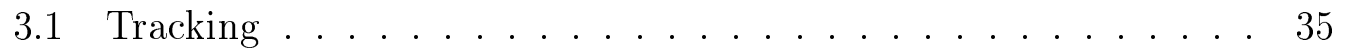

3.2 Muon identification . . . . . . . . . . . . . 36

3.3 Electron identification ................ 37

3.4 Reconstruction of the $D^{0}, D^{*+}$ mesons $\ldots \ldots . \ldots . . . . .39$

$3.4 .1 \quad D^{0}$ reconstruction $\ldots \ldots \ldots \ldots \ldots \ldots$

$3.4 .2 D^{*+}$ reconstruction . . . . . . . . . . . . 40

3.5 Reconstruction of the $D_{s}^{+}$mesons . . . . . . . . . . 45 
3.5.1 $\phi(1020) \rightarrow K^{+} K^{-}$reconstruction . . . . . . . . 45

3.5.2 $D_{s}^{+} \rightarrow \phi \pi^{+}$reconstruction ........... 45

3.6 Reconstruction of the $B$ meson decays $\ldots \ldots \ldots$. . . . . 51

4 Measurement of the $B^{-} / \bar{B}^{0}$ meson lifetimes $\quad 55$

$4.1 \quad B^{-} / \bar{B}^{0}$ Monte Carlo samples . . . . . . . . . . . . . . 55

4.1.1 Tuning of $p_{T}(B)$ spectrum . . . . . . . . . 56

4.2 Missing momentum correction . . . . . . . . . . . 58

4.3 Combinatorial Background . . . . . . . . . . . . 61

4.4 Decay length resolution . . . . . . . . . . . . . 63

4.5 Physics backgrounds . . . . . . . . . . . . 65

4.5.1 $c \bar{c}, b \bar{b}$ Monte Carlo samples . . . . . . . . . . . . 67

4.5.2 Prompt charm background . . . . . . . . . . 67

4.5.3 Bottom background . . . . . . . . . . . . . . 74

4.6 Sample Composition . . . . . . . . . . . . . . 77

4.6.1 $\pi_{s}^{+}$reconstruction efficiency .................. 77

4.6.2 Cross talk by $D^{* *} \ldots \ldots \ldots . \ldots . \ldots 79$

4.7 Lifetime fit . . . . . . . . . . . . . . . . 93

4.8 Systematic uncertainties . . . . . . . . . . . . . . 97

4.8.1 Physics backgrounds . . . . . . . . . . . . 97

4.8.2 Sample composition . . . . . . . . . . . . . 97

4.8.3 Missing momentum correction . . . . . . . . . . . . 98

4.8.4 Other sources of systematics . . . . . . . . . . 99

5 Measurement of the $\bar{B}_{s}^{0}$ meson lifetime 103

$5.1 \bar{B}_{s}^{0}$ Monte Carlo samples and missing momentum correction . . 103

5.2 Combinatorial background . . . . . . . . . . 106

5.3 Decay length resolution . . . . . . . . . . . . . . . . 109

5.4 Physics background . . . . . . . . . . . . . . 111

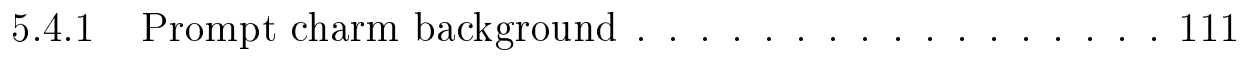

5.4.2 Bottom background . . . . . . . . . . . . 119

5.5 Lifetime extraction . . . . . . . . . . . . . . . . 122

5.6 Systematic uncertainties . . . . . . . . . . . . 125

5.6 .1 Physics background . . . . . . . . . . . 125

5.6.2 Missing momentum correction . . . . . . . . . . 127

5.6.3 Other sources of systematic uncertainties . . . . . . . 128

5.7 Ratio of the $\bar{B}_{s}^{0}$ and $\bar{B}^{0}$ meson lifetimes . . . . . . . . 134

5.8 A non-zero $\Delta \Gamma_{s} / \Gamma_{s}$ value . . . . . . . . . . 136

$6 \quad$ Results and conclusions 139 
$\begin{array}{lr}\text { A Probability density function formulae } & 147\end{array}$

A.1 Signal probability density function . . . . . . . . . . . 147

A.2 Combinatorial background modeling function . . . . . . . . 148

A.3 Function for prompt enhanced sample . . . . . . . . . . . 148

B Check of lifetime fitter using toy Monte Carlo samples 149

C Semi-analytic form of $c t_{2}$ distribution for secondary charm mesons 



\section{List of Figures}

1.1 Examples of the interaction with $W$ boson for lepton and quark case. ....................... 4

1.2 The spectator decay diagram of a $B$ meson. . . . . . . . . . 5

1.3 Weak annihilation diagrams for charm mesons. . . . . . . . 6

1.4 External (top) and internal (bottom) spectator diagrams for $B^{-}$ (left) and $\bar{B}^{0}$ (right). In the diagrams of $B^{-}$decay the final state contains the same particles, that occurs the Pauli Interference. . 7

2.1 Aerial view of the accelerator complex at the Fermi National Accelerator Laboratory. The upper ring is the Tevatron, and lower is the main injector. . . . . . . . . . . . 11

2.2 Schematic view of the accelerator complex at the Fermilab. . . . 12

2.3 Layout of the anti-proton source. . . . . . . . . . . . 14

2.4 Solid cutaway view of the CDF detector. . . . . . . . . . 15

2.5 Cut views of the CDF detector. Upper is an elevation view of the one half of the whole detector, lower is for detailed view for the quarter of the detector. . . . . . . . . . . . . 16

2.6 Schematic picture of the CDF coordinate system. . . . . . . 17

2.7 Figures of the SVX II detector. Upper figure shows a view of three barrels, and lower figure is $r$ - $\phi$ view for one barrel. Each barrel has 5 layers, and each layer has 12 wedges. . . . . . . . 19

2.8 Schematic layout of the silicon tracking system. . . . . . . . . 20

2.9 Figure of the ISL detector. . . . . . . . . . . . 20

$2.10 r$ - $\phi$ view of the COT. Upper figure is a view of east endplate. Lower figure shows three drift cells of the COT in superlayer 2. . 23

2.11 Development of an electromagnetic shower (left) and a hadronic shower (right). . . . . . . . . . . . . 25

2.12 One wedge of the CEM with its light collecting system. . . . . 26

2.13 Location of the muon detectors for Run II in azimuth $\phi$ and pseudo-rapidity $\eta \ldots \ldots \ldots$. . . . . . . . . . . . . 28 
2.14 Drawings of the CMU. In right drawing locations in $r-\phi$ and $r-z$ are shown for three modules. Left drawing is a cross section of a CMU module showing its 16 drift tube cells. . . . . . . . . 29

2.15 Configuration of the CMP. The dark gray square structure surrounding the central detector shows the CMP modules and scintillators. . . . . . . . . . . . . . 30

2.16 Architecture of the three-level pipelined and buffered trigger system used in the CDF experiment. (The numbers are for $132 \mathrm{~ns}$ operation, while actual operation is with $396 \mathrm{~ns}$ bunch spacing.)

2.17 Block chart of the trigger system for CDF experiment. The trigger Supervisor Interface (TSI) distributes signals between the trigger and the data acquisition system such as the bunch crossing signal and Level 1 and Level2 accept or reject signals. . 34

3.1 Passage of a muon through detectors. . . . . . . . . . . . 37

3.2 An event display of a typical $\ell^{-} D^{0}$ candidate. Lower figure is a close view of the upper figure around the collision point. . . . .

$3.3 M\left(K^{-} \pi^{+}\right)$plots for the $\ell^{-} D^{0}\left(D^{*+}\right.$ excluded) sample (left) and $\Delta M\left(K^{-} \pi^{+} \pi_{s}^{+}-K^{-} \pi^{+}\right)$plots for the $\ell^{-} D^{*+}$ sample. In the $M\left(K^{-} \pi^{+}\right)$plots, box histograms show events which have wrong charge correlation between lepton and kaon. In the $\Delta M$ plots, histograms for events where $\pi_{s}$ track has wrong charge are also shown. Upper and lower plots are for the muon and electron datasets, respectively.

$3.4 M\left(K^{-} \pi^{+}\right)$and $\Delta M\left(K^{-} \pi^{+} \pi_{s}^{+}-K^{-} \pi^{+}\right)$plots for the $\ell^{-} D^{0}\left(D^{*+}\right.$ excluded) and the $\ell^{-} D^{*+}$ samples (muon and electron combined dataset). . . . . . . . . . . . . . . . 4 44

3.5 A schematic drawing which shows the definition of helicity angle 4.......................... 47

3.6 The $\cos \psi$ and $M\left(\ell D_{s}^{+}\right.$) distributions for the $\ell^{-} D_{s}^{+}$(muon and electron combined) sample. Distributions for signal, sideband region events are shown as well as the difference between them.

$3.7 M\left(K^{+} K^{-}\right)$and $M\left(K^{+} K^{-} \pi^{+}\right)$plots for the $\ell^{-} D_{s}^{+}$sample. In the $M\left(K^{+} K^{-} \pi^{+}\right)$plots, points show events which have right sign charge correlation between lepton and pion, while histograms show events with wrong charge correlation. Upper and lower plots are for the muon and electron datasets respectively. . . . 49

$3.8 M\left(K^{+} K^{-}\right)$and $M\left(K^{+} K^{-} \pi^{+}\right)$plots for muon and electron combined sample. . . . . . . . . . . . . 50

3.9 Schematic view of typical semileptonic decay $B^{-} \rightarrow \ell^{-} \bar{\nu} D^{0}$, $D^{0} \rightarrow K^{-} \pi^{+}$in $x y$ plane. . . . . . . . . . . 53 
3.10 Schematic view of typical semileptonic decay $\bar{B}_{s}^{0} \rightarrow \ell^{-} \bar{\nu} D_{s}^{+}$, $D_{s}^{+} \rightarrow \phi \pi^{+}, \phi \rightarrow K^{+} K^{-}$in $x y$ plane. . . . . . . . 53

4.1 Comparison of the $B^{-} / \bar{B}^{0}$ meson transverse momentum spectra between the NDE calculation and the Run II measurement using $B \rightarrow J / \psi X$. Points show the measured spectrum, and red histogram is the NDE spectrum. Upper plot is a comparison before tuning the NDE spectrum, while lower plot is after tuning. 57

$4.2 K$ factor distributions for the muon dataset. . . . . . . . . . 59

$4.3 K$ factor distributions for the electron dataset. . . . . . . . . 60

4.4 Fit results for $c t^{*}$ of the sideband events. These shapes represent

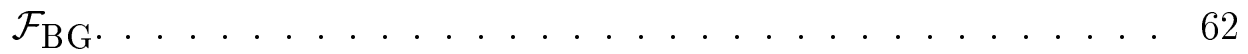

4.5 Fitting results for the prompt enhanced sample where we determine the resolution scale factor. Upper and lower plots show results for the muon and electron datasets respectively. . . . . . 64

4.6 $M(K \pi)$ plots for $\ell^{+} D^{0}$ events, which have wrong charge correlation. . . . . . . . . . . . . 66 66

4.7 Fit for $c t_{2}$ of the sideband events. These fitting results are taken as background shapes in the $f_{c}$ fits. . . . . . . . . . 71

4.8 Fitting result for the $f_{c}$ determination. In each plot green and blue curves show charm and $B$ templates respectively. Red curves describe combinatorial background shapes. . . . . . . 72

$4.9 F_{c}$ shape for $c t^{*} \neq 0$ events in the charm background, obtained from $c \bar{c}$ Monte Carlo sample. . . . . . . . . . . . . . 73

$4.10 c t^{*}$ shapes of the bottom background events obtained from the $b \bar{b}$ Monte Carlo samples. . . . . . . . . . . . . . . 76

4.11 Distributions of $M\left(K^{-} \pi^{+}\right)$and $\Delta M\left(K^{-} \pi^{+} \pi_{s}^{+}-K^{-} \pi^{+}\right)$in different $D^{0}$ transverse momentum range. These are for the muon dataset. . . . . . . . . . . . . . . 83

4.12 Same as previous figures but higher momentum range (4-5, 5$6 \mathrm{GeV} / c) \ldots \ldots \ldots \ldots \ldots . \ldots \ldots$

4.13 Same as previous figures but higher momentum range $(6-8,8-$ $10 \mathrm{GeV} / c)$.

4.14 Same as previous figures but higher momentum range (10-12, $>12 \mathrm{GeV} / c) . \ldots \ldots \ldots \ldots$
Distributions of $M\left(K^{-} \pi^{+}\right)$and $\Delta M\left(K^{-} \pi^{+} \pi^{+}-K^{-} \pi^{+}\right)$in different $D^{0}$ transverse momentum range. These are for the electron dataset. .................... 87

4.16 Same as previous figures but higher momentum range (4-5, 5$6 \mathrm{GeV} / c) \ldots \ldots \ldots \ldots \ldots \ldots$

4.17 Same as previous figures but higher momentum range (6-8, 8$10 \mathrm{GeV} / c) \ldots \ldots \ldots \ldots \ldots$ 
4.18 Same as previous figures but higher momentum range $(10-12,>$ $12 \mathrm{GeV} / c) . \ldots \ldots \ldots \ldots \ldots$

4.19 The ratio of $D^{*+} / D^{0}$ yield against $D^{0}$ meson transverse momentum. The lines show a level of plateau, which corresponds to the tracking efficiency of $99.6 \%$.

4.20 The $p_{T}$ distributions of the slow pion and the $D^{0}$ meson, and their correlation. The plots are for $\mu^{-} D^{*+}$ signal region events. Lower two correlation plots are same but with different scale. . . 92

4.21 The $c t^{*}$ distributions (points) and the fit results for the muon dataset (upper) and for the electron (lower). Blue curves show the semileptonic $B^{-} / \bar{B}^{0}$ signal components, Red curves are the combinatorial background shape. Dashed green and blue curves show charm and bottom background components, respectively. . 95

4.22 The muon and electron combined fit result. . . . . . . . . . . 96

4.23 The changes of $g_{-}$as functions of $f^{* *}$ and $P_{V}$ under several conditions for muon dataset. In the left plots $P_{V}$ is set to the default value 0.651 . Upper plots are for the $p_{T}\left(D^{0}\right)<8 \mathrm{GeV} / c$ region, and lower plots are for the $p_{T}\left(D^{0}\right)>8 \mathrm{GeV} / c$ region. The lifetime ratio $\tau\left(B^{-}\right) / \tau\left(\bar{B}^{0}\right)$ is set to be unity. . . . . . . . 101

4.24 Same as the previous figures but for the electron dataset. . . . . 102

5.1 The $K$ factor distributions for the muon dataset (top) and the electron dataset (bottom). . . . . . . . . . . . 105

5.2 Fit results for the $c t^{*}$ distributions of the sideband events. Overlaid curves show $\mathcal{F}_{B G}$, which represent the combinatorial background shape to be used in the final lifetime fit. . . . . . . . . 108

5.3 Fit results for the prompt enhanced samples when we determine the resolution scale factor. . . . . . . . . . . . . . 110

5.4 Results of the $f_{c}$ fit for muon and electron datasets. Top plots are fit results for the signal region. Bottom plots are for the sideband fit results to determine the combinatorial background shape. In the top plot, green and blue curve show the prompt charm and $\bar{B}_{s}^{0}$ templates, respectively. Red curve describes the combinatorial background shape. . . . . . . . . . . 117

5.5 Non-zero $c t^{*}$ distributions for prompt $c \bar{c}$ events. . . . . . . . . 118

5.6 The $c t^{*}$ distributions for bottom background events obtained from the Monte Carlo simulation for muon and electron datasets. Colored histograms show contributions from each processes, and points show sum of all the contributions. Overlaid curves are fit results for the total background shapes and represent the bottom background template functions. . . . . . . . . . . . . . 121 
5.7 The $c t^{*}$ distributions and lifetime fit results. Left plot is for the muon, and right plot is for the electron dataset. Overlaid curves are the fit functions. Blue curves show the semileptonic $\bar{B}_{s}^{0}$ signal components, red curves are the combinatorial background function. Dashed green and blue curves show the charm and bottom background components, respectively. . . . . . . . 123

5.8 The lifetime fit result for the muon and electron combined dataset. Red curve shows the sum of all the backgrounds (combinatorial and physics backgrounds). . . . . . . . . . . . . . . 124

5.9 Comparison of the $K$ factor distributions with different generators (EvtGen and QQ), for the muon and electron datasets. . . 132

5.10 Comparison of the distributions of the momentum ratio $K$ for different semileptonic decay modes, $\bar{B}_{s}^{0} \rightarrow \ell^{-} \bar{\nu} D_{s}^{+}, \rightarrow \ell^{-} \bar{\nu} D_{s}^{*+}$ and $\rightarrow \ell^{-} \bar{\nu} D_{s}^{* *+}$. . . . . . . . . . . . . . . 133

5.11 Result of the two exponential fits with $\Delta \Gamma_{s} / \Gamma_{s}=0.37 . \ldots 137$

6.1 A comparison of $\tau\left(B^{+}\right)$measured by other experiments and this analysis. The result of this analysis is included in the world average. . . . . . . . . . . . . . . . . 141

6.2 A comparison of $\tau\left(B^{0}\right)$ measured by other experiments and this analysis. The result of this analysis is included in the world average. . . . . . . . . . . . . . . . . . 142

6.3 A comparison of $B^{0} / B^{+}$lifetime ratio with other measurements and this analysis. The result of this analysis is included in the world average. . . . . . . . . . . . . . . 143

6.4 A comparison of $\tau\left(B_{s}^{0}\right)$ measured by other experiments and this analysis. The result of this analysis is not included in the world average. . . . . . . . . . . . . . . . . 144

6.5 Measurements of the ratio of the $\bar{B}_{s}^{0}$ and $\bar{B}^{0}$ meson lifetimes from other experiments and this analysis. Only those measurements which quote the ratio explicitly are listed. . . . . . . . . 145

B.1 Pull distributions to check lifetime fitter using toy Monte Carlo samples. . . . . . . . . . . . . . . 150

C.1 Distributions of variable $c t_{2}$ for prompt and secondary charm mesons (curves). Results of toy Monte Carlo experiments are overlaid as histograms. 



\section{List of Tables}

1.1 Summary of fundamental fermions. Each column corresponds to each generation. Electric charge is in unit of $e=1.602 \times 10^{-19}$ C. 2

1.2 The three interactions and mediators described by the standard model ....................... 2

1.3 World average of lifetimes in 2004 for various $B$ and $D$ mesons. $\quad 5$

1.4 Ratios of the different $B$ meson lifetimes predicted with HQE. $f_{B}$ is a decay constant of the $B$ meson. . . . . . . . . 8

2.1 The COT design parameters. . . . . . . . . . . . 22

2.2 Summary table of the position resolution of the various components of the CDF tracking system. . . . . . . . . . 22

3.1 Summary of signal region definitions and yields for the $\ell^{-} D^{0}$ $\left(D^{*+}\right.$ excluded) and the $\ell^{-} D^{*+}$ samples. . . . . . . . .

3.2 Summary of sideband region definitions and yields for the $\ell^{-} D^{0}$ ( $D^{*+}$ excluded) and the $\ell^{-} D^{*+}$ samples. . . . . . . . . . 41

3.3 Summary of signal, sideband region definitions and event yields for the $\ell^{-} D_{s}^{+}$samples. . . . . . . . . . . . 47

4.1 Fit results for the $c t^{*}$ shapes of the sideband events. Unit of $\sigma$

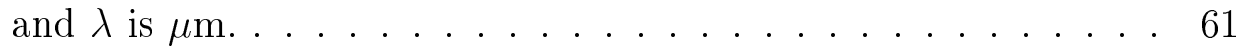

4.2 Fitting results for the prompt enhanced samples on the resolution scale factor determination. Unit of $\lambda_{ \pm}$is $\mu \mathrm{m} . \ldots . . . .63$

4.3 Summary of the charm background fraction in each sample. . . 69

4.4 The $\mathcal{F}_{c}^{\text {non-zero }}$ parameters. Unit of $\sigma$ and $\lambda_{ \pm}$is $\mu \mathrm{m} . \ldots 70$

4.5 A summary of the $b \bar{b}$ Monte Carlo events with RS track $+D^{0}$ final states after kinematic cuts. $\ell^{-}$is $\mu^{-}$or $e^{-}$, and $\bar{B}$ denotes either $B^{-}$or $\bar{B}^{0}$. . . . . . . . . . . . . . 75

4.6 Fit results for four types of bottom background. $\ell^{-} D^{0}$ and $\ell^{-} D^{*+}$ denote the non-semileptonic $B$ events here. Unit of $\sigma, \lambda$

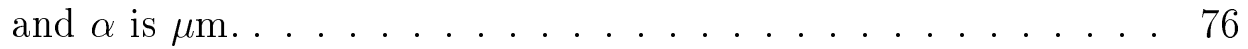

4.7 The yields of the $D^{*+}$ and $D^{0}$ signal in each $p_{T}\left(D^{0}\right)$ region. . . 78 
4.8 Summary of the slow pion reconstruction efficiency in each $D^{0}$ transverse momentum region. . . . . . . . . . . 78

4.9 Composition in full simulation samples for the muon dataset, $p_{T}\left(D^{0}\right)<8 \mathrm{GeV} / c$. NR means Non-Resonant decay, which is also counted as a $D^{* *}$ state. In the lower table, ${ }^{3} P_{0}$ states decay only to $D \pi,{ }^{3} P_{1},{ }^{1} P_{1}$ decay only to $D^{*} \pi$. ${ }^{3} P_{2}$ and Non-Resonant states decay to both $D \pi$ and $D^{*} \pi \ldots \ldots \ldots$. . . . . 80

4.10 Same with the previous table, but for $p_{T}\left(D^{0}\right)>8 \mathrm{GeV} / c \ldots \ldots 80$

4.11 Same but for electron dataset, $p_{T}\left(D^{0}\right)<8 \mathrm{GeV} / c \ldots \ldots$. . . . 81

4.12 Same for electron dataset, but $p_{T}\left(D^{0}\right)>8 \mathrm{GeV} / c \ldots . . . .81$

4.13 Composition in full simulation samples after considering the slow pion reconstruction efficiency. . . . . . . . . . . 82

4.14 Table of $g_{-}$( $B^{-}$fraction) in each sample with $\tau\left(B^{-}\right) / \tau\left(\bar{B}^{0}\right)=1.82$

4.15 Fitting probabilities calculated from each fit result. . . . . . . . 94

4.16 The $g_{-}\left(B^{-}\right.$fraction $)$in each sample determined by the combined fit. . . . . . . . . . . . . . . . . . 94

4.17 A summary of $g_{-}$in each sample and each $D^{0}$ transverse momentum region evaluated when $f^{* *}$ and $P_{V}$ are varied. $\tau\left(B^{-}\right) / \tau\left(\bar{B}^{0}\right)$ is set to be unity in this table. . . . . . . . . . . . . . 99

4.18 A summary of the lifetime fit when $f^{* *}, P_{V}, \epsilon\left(\pi_{s}\right)$ are varied. In the column $\epsilon\left(\pi_{s}\right)$ "low" and "high" mean regions of $p_{T}\left(D^{0}\right)$ lower and greater than $8 \mathrm{GeV} / c$, respectively. . . . . . . . 100

4.19 A summary of the systematic uncertainties in the $B^{-} / \bar{B}^{0}$ meson lifetime measurement. . . . . . . . . . . . . 100

5.1 Fit results for the $c t^{*}$ shapes of the sideband events. Unit of $\sigma$

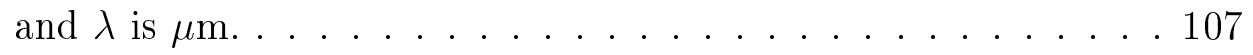

5.2 Results of the resolution scale factor determination using promptenhanced sample . . . . . . . . . . . . . 109

5.3 Fraction of prompt charm background obtained from fit to $c t_{2}$ distribution. . . . . . . . . . . . . . . 113

5.4 The $\mathcal{F}_{c}^{\text {non-zero }}$ parameters. Unit of $\sigma$ and $\lambda_{ \pm}$is $\mu \mathrm{m} . \ldots 116$

5.5 Estimate of bottom backgrounds using Monte Carlo calculation. $N_{\text {events }}$ is the number of events which survive all cuts, weighted for the $p_{T}^{B}$ spectra and the branching fractions of forced decay modes. . . . . . . . . . . . . . . 120

$5.6 c t^{*}$ shape of the bottom background events. . . . . . . . . 120

5.7 Fitting probabilities calculated from each fit result. . . . . . . 123 
5.8 Summary of fit results performed for evaluation of systematic uncertainties. Symbols $\mu$ and $e$ denote the muon and electron data sets. If they both appear in a single row, the parameter is considered correlated between the two data sets and is changed simultaneously for the two data sets. Otherwise it is considered independent. . . . . . . . . . . . . . . 130

5.9 Summary of systematic uncertainties in the $\bar{B}_{s}^{0}$ meson lifetime measurement. . . . . . . . . . . . . . 131

5.10 Summary of systematic uncertainties in the $\bar{B}_{s}^{0} / \bar{B}^{0}$ lifetime ratio. 135

5.11 Results of the two exponential fits with fixed $\Delta \Gamma_{s} / \Gamma_{s}$ values $\left(\Delta \Gamma_{s}=0.37_{-0.20}^{+0.17}[1]\right)$. Quoted uncertainties are statistical only. 137 



\section{Chapter 1}

\section{Introduction}

What are the ultimate elements of matter? And how the interactions occur between them? The elementary particle physics is a study to answer these questions. In the last century the elementary particle physics showed significant progress. Now the total theoretical system is assembled as "The Standard Model".

In the standard model, particles can be classified according to their spin statistics as either bosons that have integer spin, or fermions that have half integer spin. Quarks and leptons, carrying spin 1/2, belong to the latter group and are thought to be the fundamental constituents of matter in the standard model. Today we know 12 different types of fundamental fermions, six leptons and six quarks. For each of these particles there exists an antiparticle with the same mass but opposite electric charge. Table 1.1 summarizes the fundamental fermions. The fermions are classified into three "generations" as shown in Table 1.1. Fermions in upper generation have heavier masses (note that it is still not surely true for neutrinos). In addition to the fundamental fermions, the standard model includes a description of the force-carrying particles, the gauge bosons, and the force or interaction they mediate between fermions and themselves. Four fundamental forces are known today. They are, in order of decreasing strength, the strong force, the electromagnetic force, the weak force and the gravitational force. While the gravitational force is not included in the standard model, the electromagnetic and weak force find a unified description reducing the four interactions to three. Table 1.2 lists the interactions treated in the standard model together with their respective force mediating particles. 


\begin{tabular}{cc|ccc}
\hline \hline & $\begin{array}{c}\text { Electric } \\
\text { charge }\end{array}$ & \multicolumn{2}{|c}{ Symbols } \\
\hline Quarks & $+\frac{2}{3}$ & $u$ & $c$ & $t$ \\
& $-\frac{1}{3}$ & $d$ & $s$ & $b$ \\
\hline Leptons & 0 & $\nu_{e}$ & $\nu_{\mu}$ & $\nu_{\tau}$ \\
& -1 & $e^{-}$ & $\mu^{-}$ & $\tau^{-}$ \\
\hline \hline
\end{tabular}

Table 1.1: Summary of fundamental fermions. Each column corresponds to each generation. Electric charge is in unit of $e=1.602 \times 10^{-19} \mathrm{C}$.

\begin{tabular}{cccc}
\hline \hline Force & Mediator & Electric charge & Mass $\left(\mathrm{GeV} / c^{2}\right)$ \\
\hline \hline strong & gluon $(\mathrm{g})$ & 0 & 0 \\
\hline electromagnetic & photon $(\gamma)$ & 0 & 0 \\
\hline Weak & $W$ boson $\left(W^{ \pm}\right)$ & \pm 1 & $80.425 \pm 0.038$ \\
& $Z$ boson $\left(Z^{0}\right)$ & 0 & $91.1768 \pm 0.0021$ \\
\hline \hline
\end{tabular}

Table 1.2: The three interactions and mediators described by the standard model

\subsection{Weak interaction and CKM matrix}

In the framework of the standard model, weak and electromagnetic interactions appear in consequence of the requirement that the Lagrangian is invariant under $S U(2)_{L}$ and $U(1)_{Y}$ gauge transformations. Here $L$ stands for left handed, expressing that the weak interaction is parity violating and thus left and right handed components of the fields should be treated separately. $Y$ denotes the weak hypercharge defined as $Y / 2=Q_{\mathrm{em}}-I_{3}$, where $I_{3}$ is the third component of the weak isospin $I$ and $Q_{\mathrm{em}}$ is the electromagnetic charge.

An example of the weak interaction diagram for the electron is shown in Figure 1.1 left side. In this diagram the electron weak current is written as

$$
J_{e}^{\mu}=\bar{u}_{\nu_{e}} \gamma^{\mu} \frac{1}{2}\left(1-\gamma^{5}\right) u_{e}
$$

This equation indicates that at the interaction with the $W$ boson, electron always transits to $\nu_{e}$, which is in the same weak isospin doublet. However in the weak interaction of the quark, the situation is a bit different. A diagram of the weak interaction of the up-type quark is shown in Figure 1.1 right side. The corresponding quark current is described as

$$
J_{\ell}^{\mu}=\bar{u}_{u} \gamma^{\mu} \frac{1}{2}\left(1-\gamma^{5}\right) u_{d^{\prime}},
$$


where $d^{\prime}$ is a mixture of the down-type quarks, $d^{\prime}=V_{u d} d+V_{u s} s+V_{u b} b$. It indicates that if the energy conservation law permits, transition between the up and down type quarks over the generation can occur. In general, Lagrangian for quark current is described as follows :

$$
\mathcal{L}_{\mathrm{CC}}=\frac{g}{\sqrt{2}} \sum_{i, j=1}^{3} \bar{u}_{i} \gamma^{\mu} \frac{1}{2}\left(1-\gamma^{5}\right) V_{i j} d_{j} W_{\mu}^{+}+\text {h.c. },
$$

where $i$ and $j$ denote generation of the up and down type quarks respectively. The $3 \times 3$ matrix $V$, appeared in the formula, is called Cabbibo-KobayashiMaskawa (CKM) matrix [2] and describes the strength of quark-quark couplings across the generations in the weak interaction.

$$
V=\left(\begin{array}{ccc}
V_{u d} & V_{u s} & V_{u b} \\
V_{c d} & V_{c s} & V_{c b} \\
V_{t d} & V_{t s} & V_{t b}
\end{array}\right)
$$

If the CKM matrix is diagonal, then the inter-transition of the up and down type quarks can not be happened. Experimental results of the CKM matrix measurement show that CKM matrix is close to a unity matrix, but off-diagonal elements have non-zero values. Following is the magnitude of the CKM matrix elements which are measured experimentally [1].

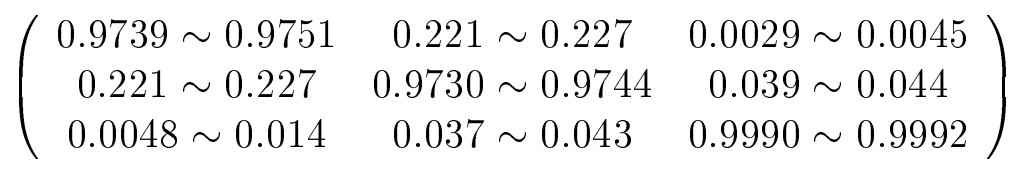

\subsection{Theoretical overview of the $B$ meson life- times}

The $B$ mesons are bound states of an anti- $b$ quark and a lighter quark. In this analysis we measure lifetimes of three different species of $B$ mesons, namely $B^{-}(b \bar{u}), \bar{B}^{0}(b \bar{d})$, and $\bar{B}_{s}^{0}(b \bar{s})$. (The final $B$ meson, $B_{c}^{-}(b \bar{c})$ is found in CDF Run I, and study of its property is ongoing elsewhere in CDF Run II [3][4].)

The binding between two quarks in a $B$ meson is provided by the strong force. At the same time, ground state $B$ mesons can only decay via the weak interaction, in most cases by means of the $b \rightarrow W q$ sub-process, where $q$ is either a $c$ or a $u$ quark and the emitted $W$ boson is highly virtual. Studying $B$ mesons gives physicists a handle on otherwise inaccessible elements of the CKM matrix elements $V_{c b}, V_{u b}, V_{t d}$ and $V_{t s}$. Also, measuring the $B$ meson lifetimes provides good tests of the Heavy Quark Expansion (HQE), which predicts lifetime difference between different $B$ or $D$ meson species. In this section we briefly look over the theoretical overview of the $B$ meson lifetimes. 

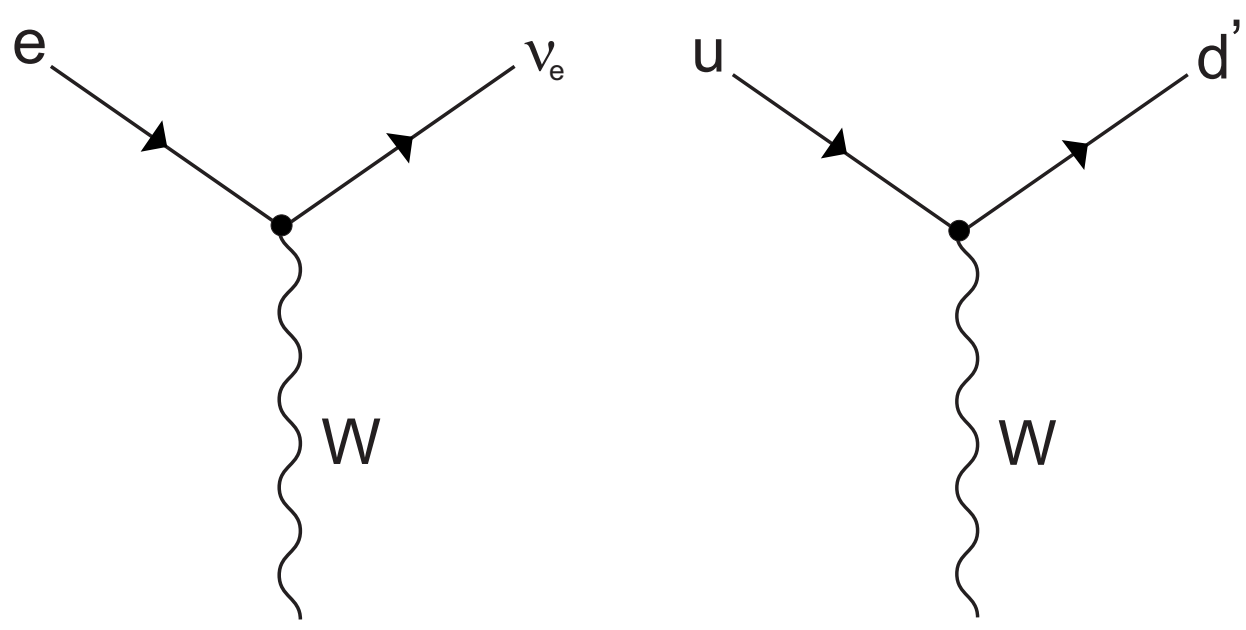

Figure 1.1: Examples of the interaction with $W$ boson for lepton and quark case.

\subsubsection{Spectator model}

In the naive spectator model, it is assumed that the light quarks in a heavy hadron do not affect the decay of the heavy quark. The lifetimes of weakly decaying hadrons that contain the same heavy quark are thus equal in this model, regardless of the flavor of the accompanying quarks. Figure 1.2 shows the Feynman diagram for the spectator decay of $B$ meson. Following the muon decay, the spectator model can give a very rough estimate of the expected lifetimes for charm and bottom hadrons. In muon decays the virtual $W$ boson decays to $e \nu_{e}$ since that is the only kinematically allowed channel. However in charm decays the $W$ boson has five possibilities to decay $\left(e \nu_{e}, \mu \nu_{\mu}, u \bar{d} \times 3\right.$ colors). In bottom decays the number of possible decay products increases to nine. Furthermore the quark-quark couplings and the mass of the decaying particle must be adjusted when starting from the muon decay formula (Since this is very rough estimate, we here neglect phase space and QCD correction factors). With these adjustments the lifetime of bottom hadrons can be estimated as,

$$
\Gamma_{b} \approx \Gamma_{\mu} \times \frac{9 V_{c b}^{2} M_{b}^{5}}{M_{\mu}^{5}} \rightarrow \tau_{b}=\frac{1}{\Gamma_{b}} \approx 1.3-1.7 \mathrm{ps}
$$

with $M_{b}=4.2 \mathrm{GeV} / c^{2}$ and $V_{c b}=0.039-0.044$,

Soon after the simple spectator model was proposed it became apparent, particularly in the charm sector, that other diagrams have to be included in the description of decays of heavy hadrons to explain the measured lifetime differences between hadrons containing different spectator quarks. The primary 


\begin{tabular}{cccc}
\hline \hline \multicolumn{2}{c}{$D$ mesons } & \multicolumn{2}{c}{$B$ mesons } \\
Meson & lifetime [ps] & Meson & lifetime $[\mathrm{ps}]$ \\
\hline$D^{+}$ & $1.040 \pm 0.007$ & $B^{-}$ & $1.671 \pm 0.018$ \\
$D^{0}$ & $0.410 \pm 0.002$ & $\bar{B}^{0}$ & $1.536 \pm 0.014$ \\
$D_{s}^{+}$ & $0.490 \pm 0.009$ & $\bar{B}_{s}^{0}$ & $1.461 \pm 0.057$ \\
\hline \hline
\end{tabular}

Table 1.3: World average of lifetimes in 2004 for various $B$ and $D$ mesons.

indication was measurements of the lifetime of the $D^{+}$and the $D^{0}$ meson which yielded $\tau\left(D^{+}\right) / \tau\left(D^{0}\right) \sim 2.5$. Table 1.3 lists the current results for lifetime measurements of various $b$ and $c$ hadrons [1]. The necessity for the inclusion of non-spectator diagrams is made obvious by the spread of the measured lifetimes.

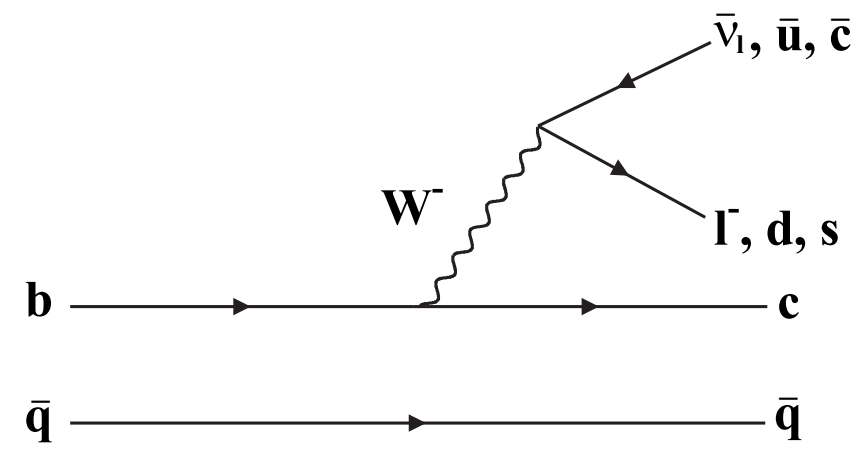

Figure 1.2: The spectator decay diagram of a $B$ meson.

\subsubsection{Non-spectator effects}

There are several decay processes that directly involve the spectator quarks and that can contribute to the decay of heavy hadrons even though they may be suppressed with respect to the spectator processes. The good examples of the non-spectator effects are seen in decays of charm mesons as well as in the $B$ meson decays. Figure 1.3 shows non-spectator diagrams for charm mesons for a process referred as Weak Annihilation (WA). This process is sometimes also referred to as $W$-exchange and $W$-annihilation. WA contributes to the Cabbibo allowed decay only of $D^{0}$, while for $D^{+}$there is no WA diagram at 
the Cabbibo allowed level. Thus WA should shorten the $D^{0}$ lifetime compared to that of $D^{+}$.

Second contribution to lifetime differences comes from interference effects between decay diagrams, referred to as Pauli Interference (PI). The PI appears in the $B$ meson decay diagrams as well as the decays of charm mesons. Figure 1.4 shows the diagrams for PI for the case of a $B^{-}$and a $\bar{B}^{0}$ meson. The upper diagram is in each case an external spectator diagram, while the lower diagram represents the internal spectator decay. In the case of $B^{-}$both such diagrams yield the same final state, while in the case of $\bar{B}^{0}$ the final states differ. The PI turns out to be destructive, which again prolongs the lifetime of $B^{-}\left(D^{+}\right.$for charm meson case) over the lifetime of $\bar{B}^{0}$ ( $D^{0}$ for charm case), and is thought to be the dominant factor leading to the lifetime differences. However those phenomenological considerations can yield at best a hierarchy of different $B$ meson lifetimes such as

$$
\tau\left(B^{-}\right)>\tau\left(\bar{B}^{0}\right) \approx \tau\left(\bar{B}_{s}^{0}\right) .
$$

They cannot indicate how the corrections scale with the heavy quark mass for instance, although the naive assumption is that corrections are smaller for the heavier bottom hadrons. Today however there exists a theoretical apparatus, the heavy quark expansion, that enables concrete predictions for lifetime ratios that can be tested experimentally.
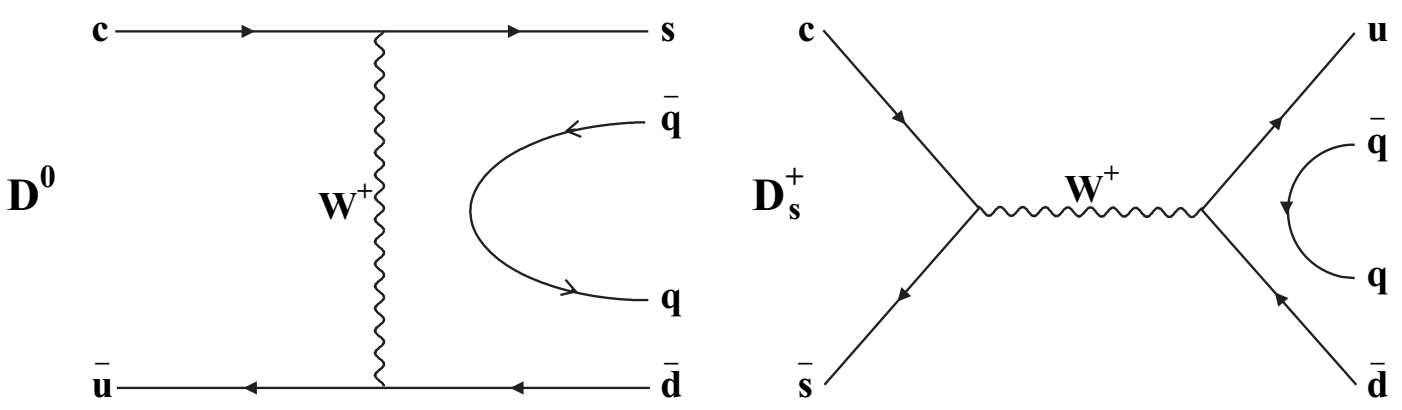

Figure 1.3: Weak annihilation diagrams for charm mesons.

\subsubsection{Heavy Quark Expansion}

Calculating weak decays of heavy hadron is a difficult task owing to the nontrivial interplay of strong and electroweak forces. Unlike in the case of electroweak processes, all orders of perturbation theory have to be considered in QCD. Various theoretical approaches are today available for the calculation 

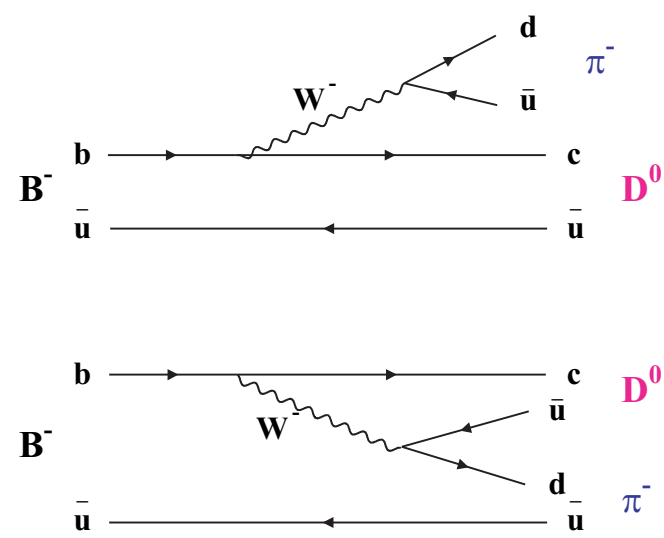
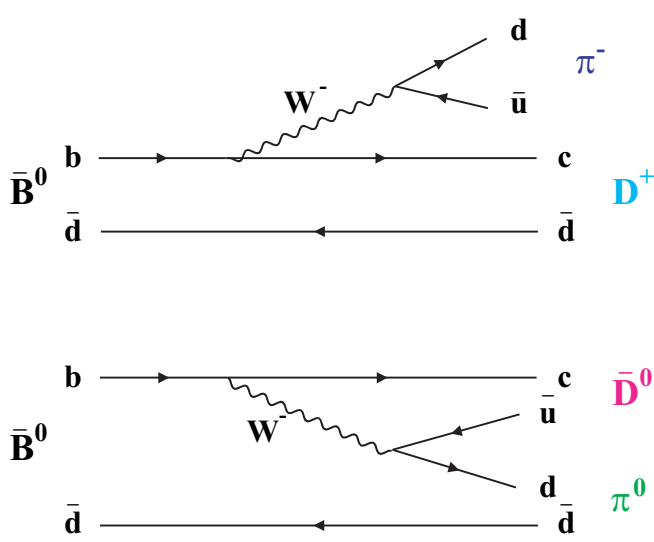

Figure 1.4: External (top) and internal (bottom) spectator diagrams for $B^{-}$ (left) and $\bar{B}^{0}$ (right). In the diagrams of $B^{-}$decay the final state contains the same particles, that occurs the Pauli Interference.

of a number of aspects of heavy quark physics. Inclusive $b$ decays are treated within the framework of the Heavy Quark Expansion, which is basically an expansion in inverse powers of the heavy quark mass. As $m_{b} \gg \Lambda_{\mathrm{QCD}}$ the inverse quark mass provides a useful expansion parameter. The HQE is based on an operator product expansion (OPE), a method to disentangle long and short distance contributions to the decay amplitudes.

The starting point in the HQE is the total decay rate $\Gamma_{H}$ of a heavy hadron,

$$
\Gamma_{H}=\frac{1}{2 m_{H}}\langle H|\mathbf{T}| H\rangle \equiv\langle T\rangle
$$

The transition operator $\mathbf{T}$,

$$
\mathbf{T}=\operatorname{Im} i \int d^{4} x T H_{e f f}(x) H_{e f f}(0),
$$

is the imaginary part of the forward scattering amplitude $H \rightarrow H$ under the action of the effective weak Hamiltonian $H_{\text {eff }}$ [5]. The application of OPE results in a series of local operators. The coefficients of those operators are suppressed by increasing powers of $1 / m_{b}$. A combination with heavy quark effective theory yields then the following important results.

- $\Gamma_{H}=\Gamma_{b}$ at leading order in the HQE. At leading order the total decay rate of a $b$ flavored hadron equals the rate of the free $b$-quark decay regardless of the additional constituent quarks. 


\begin{tabular}{cc}
\hline \hline & HQE prediction \\
\hline$\tau\left(B^{-}\right) / \tau\left(B^{0}\right)$ & $1+0.05\left(f_{B} / 200 M e V\right)^{2}$ \\
$\tau\left(\bar{B}_{s}^{0}\right) / \tau\left(\bar{B}^{0}\right)$ & $1 \pm O(0.01)$ \\
\hline \hline
\end{tabular}

Table 1.4: Ratios of the different $B$ meson lifetimes predicted with HQE. $f_{B}$ is a decay constant of the $B$ meson.

- The first correction term appears at order $1 / m_{b}^{2}$ and concerns the recoil motion of the heavy quark and interactions of the light hadronic cloud with the decay quark spin.

- The effects from non-spectator diagrams such as WA and PI are present through contributions at order $1 / m_{b}^{3}$.

The quantitative predictions for $B$ meson lifetimes are provided with the HQE calculation [6]. The predictions for the $B$ meson lifetime ratios are shown in Table 1.4. These predictions are very good to be tested by the actual measurements.

\subsection{Motivation of the $B$ meson lifetime mea- surement}

As discussed previously, measuring the lifetimes of different $B$ hadron species has great importance and of interest. First motivation is to check the HQE predictions for the $B$ meson lifetimes. Besides to test the HQE, there is no doubt that the measuring the $B$ meson lifetimes is essentially important, because the lifetime of the $b$ quark directly relates to the CKM matrix element $V_{c b}$. Totally the $B$ meson lifetime measurement gives a good probe to the weak decay mechanics of the heavy hadrons. Moreover, especially measuring the $\bar{B}_{s}^{0}$ meson lifetime is important for $B_{s}^{0} \bar{B}_{s}^{0}$ oscillation, which is not observed yet. $\Delta m_{s}$, mass difference of the two mass eigenstates of the $B_{s}^{0}$ meson, is lead from the $B_{s}^{0} \bar{B}_{s}^{0}$ oscillation frequency and can give strong constraint to the CKM matrix element $V_{t s}$.

Since there are such important motivations with the $B$ meson lifetimes, several experiments have been performed the lifetime measurements. For the $B^{-}$and $\bar{B}^{0}$ mesons, the lifetimes and its ratio are measured with various decay modes by CDF Run I [7][8], SLD [9], LEP experiments [10]-[17], and $B$ factories [18]-[22],[23]. Especially the $B$ factories (Belle and Babar) report precise results of the lifetimes and ratio with uncertainty of $1-2 \%$. Also, it is extensively being 
measured in Tevatron Run II, both in CDF [24]-[26] and D $\emptyset$ [27]-[29]. A good summary of current experimental results is found in [39]. However for $\bar{B}_{s}^{0}$, it is relatively harder to create the $\bar{B}_{s}^{0}$ than $B^{-}$and $\bar{B}^{0}$, because it is necessary for $\bar{B}_{s}^{0}$ creation to pull a $s \bar{s}$ quark pair up from the vacuum. Due to this reason, the $\bar{B}_{s}^{0}$ lifetime measurement is still limited by statistical uncertainty [1][39]. In past, three of four experiments at LEP performed the $B_{s}^{0}$ lifetime measurement with $10 \%$ of uncertainty [30]-[35]. The CDF also measured the $\bar{B}_{s}^{0}$ lifetime in Run I [36][37] and Run II [24][26]. DØ recently reported the $\bar{B}_{s}^{0}$ lifetime results in Run II [27][38] but yet preliminary. Since the $B$ factories can not create the $\bar{B}_{s}^{0}$ meson, currently the Tevatron is only the collider where the $\bar{B}_{s}^{0}$ lifetime and other properties can be measured[40]. The semileptonic decay especially provides a larger amount of $\bar{B}_{s}^{0}$ events than other exclusive decay modes. However there are some difficulties specific to the semileptonic decay. First one is a missing momentum due to neutrino and other particles. Second one is physics backgrounds. For the $B^{-} / \bar{B}^{0}$ semileptonic decays, there is a complexity of $B$ component mixture with the same final state. These issues are discussed in detail in Chapter 4 and 5 . In spite of these difficulties, the $B$ meson lifetime measurement with semileptonic decays takes on advantage of large statistics in CDF Run II.

\subsection{Analysis outline}

We measure the $B^{-}, \bar{B}^{0}$ and $\bar{B}_{s}^{0}$ meson lifetimes using partially reconstructed semileptonic decays. Throughout this thesis, we apply a notation that decay processes include also their charge conjugates. Following semileptonic decay processes are used for this measurement :

$$
\begin{aligned}
B^{-} / \bar{B}^{0} & \rightarrow \ell^{-} \bar{\nu} D^{0} X \\
B^{-} / \bar{B}^{0} & \rightarrow \ell^{-} \bar{\nu} D^{*+} X \\
\bar{B}_{s}^{0} & \rightarrow \ell^{-} \bar{\nu} D_{s}^{+} X,
\end{aligned}
$$

where $\ell^{-}$denotes either a muon or electron.

The data are collected with the $8 \mathrm{GeV}$ single lepton triggers. Corresponding integrated luminosity is about 260 and $360 \mathrm{pb}^{-1}$ for the $B^{-} / \bar{B}^{0}$ and $\bar{B}_{s}^{0}$ lifetime analyses, respectively. With the single lepton triggers, events which contain a muon or electron with a transverse momentum greater than $8 \mathrm{GeV} / c$ are selected. During the data taking period, events taken with the $8 \mathrm{GeV}$ electron trigger are partly thinned out by the Level-2 trigger (so-called "prescaling"), since the electron trigger had a large rate and total data acquisition capability is limited.

For the acquired lepton candidates, further lepton identification cuts are applied to improve purity of the $B$ semileptonic decay signal. 
Confirming that a lepton candidate is associated in the event, three types of charm mesons are fully reconstructed. Following exclusive decay modes are used for the charm meson reconstruction :

$$
\begin{aligned}
D^{*+} & \rightarrow D^{0} \pi_{s}^{+} \\
D^{0} & \rightarrow K^{-} \pi^{+} \\
D_{s}^{+} & \rightarrow \phi \pi^{+}, \text {followed by } \phi \rightarrow K^{+} K^{-},
\end{aligned}
$$

where $\pi_{s}^{+}$denotes a slow pion from $D^{*+}$ to distinguish it from $\pi^{+}$from $D^{0}$ decay. Species of the reconstructed charm meson identifies the parent $B$ meson species. However in the $B^{-} / \bar{B}^{0}$ semileptonic decays, both mesons decay into the identical final state, lepton $+D^{0}$. To disentangle this mixture of the $B$ components in the $D^{0}$ sample, we adopt the following method: We split the inclusive $D^{0}$ sample according to the $D^{*+} \rightarrow D^{0} \pi_{s}^{+}$signal. The inclusive $D^{0}$ sample is split into the two samples of $D^{0}$ mesons which are from the $D^{*+}$ meson and not from $D^{*+}$. The $D^{*+}$ sample is dominated by the $\bar{B}^{0}$ component, and the $D^{0}$ sample after excluding the $D^{*+}$ events is dominated by the $B^{-}$ component. Consequently we have six mutually independent lepton + charm samples, namely $\ell D^{0}\left(D^{*+}\right.$ excluded), $\ell D^{*+}$, and $\ell D_{s}^{+}$, where $\ell$ is either a muon or electron.

We start the lifetime analysis from $B^{-} / \bar{B}^{0}$ lifetime measurement using $\ell D^{0}$ ( $D^{*+}$ excluded) and $\ell D^{*+}$ samples. Fraction of $B^{-} / \bar{B}^{0}$ components in each sample is estimated using a Monte Carlo simulation. From the lepton + charm meson pairs, we reconstruct the $B$ meson decay points and measure decay lengths. We need to interpret the decay length to the proper $B$ decay time in the $B$ meson rest frame. However, since the $B$ meson momentum is partially reconstructed in semileptonic decays, we can not fully transfer the decay length to the decay time. To correct this missing momentum effect, we utilize the Monte Carlo simulation sample. Then we estimate contributions of backgrounds. There are three types of backgrounds. One is combinatorial background, which consists of random combination of tracks which do not come from $B$ semileptonic decays. The rests are prompt charm and bottom backgrounds, which associate a real charm meson and lepton, not coming from the $B$ semileptonic decays. One remaining thing we need to consider is the resolution of the measured decay length. Since wrong estimation of the resolution biases the lifetime measurement, the decay length resolution should be properly evaluated. Including contributions from these factors, we extract the $B$ meson lifetimes using unbinned likelihood fit. After obtaining the lifetimes, systematic uncertainties from various sources are evaluated.

Once we extract the $B^{-} / \bar{B}^{0}$ lifetimes, we move to the $\bar{B}_{s}^{0}$ lifetime analysis. The general analysis strategy is basically same as the $B^{-} / \bar{B}^{0}$ lifetime analysis, but there is no complexity of the $B$ components mixture in $\bar{B}_{s}^{0}$ lifetime analysis. 


\section{Chapter 2}

\section{Experimental Apparatus}

The Collider Detector at Fermilab (CDF) is a high energy physics experiment designed to study interactions of elementary particles. The CDF is located on the Tevatron collider which provides colliding proton and anti-proton beams at the Fermi National Accelerator Laboratory (Fermilab), located about 60 $\mathrm{km}$ west of Chicago in Illinois, USA. Figure 2.1 shows an aerial view of the Fermilab and the accelerator complex.

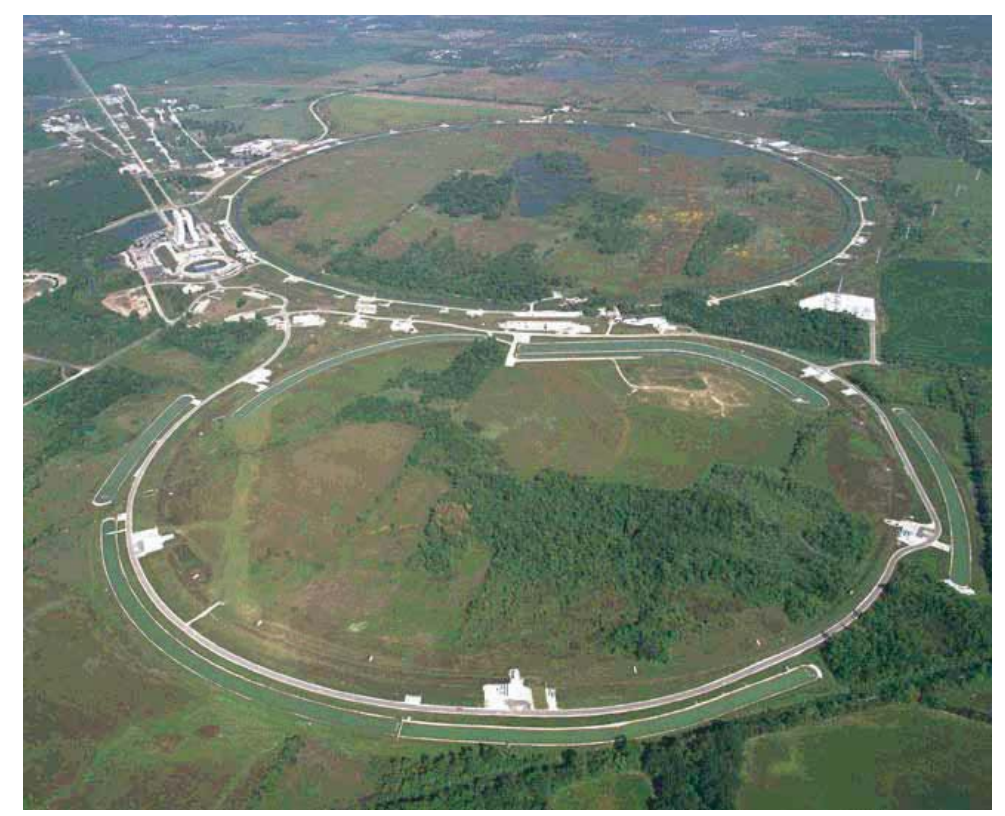

Figure 2.1: Aerial view of the accelerator complex at the Fermi National Accelerator Laboratory. The upper ring is the Tevatron, and lower is the main injector. 


\subsection{Accelerator complex}

The Tevatron collider is the final stage in a chain of accelerators. With the accelerator complex, protons and anti-protons are created and gradually accelerated to collide at two designated points [41][42]. The center-of-mass energy of the $p \bar{p}$ collision is $1.96 \mathrm{TeV}$. The accelerator complex consists of several components. A schematic view of the accelerator complex is shown in Figure 2.2. The proton and anti-proton beams are created and accelerated in the chain of accelerators step-by-step. This section briefly describes how the proton and anti-proton beams are provided and accelerated in the accelerator complex.

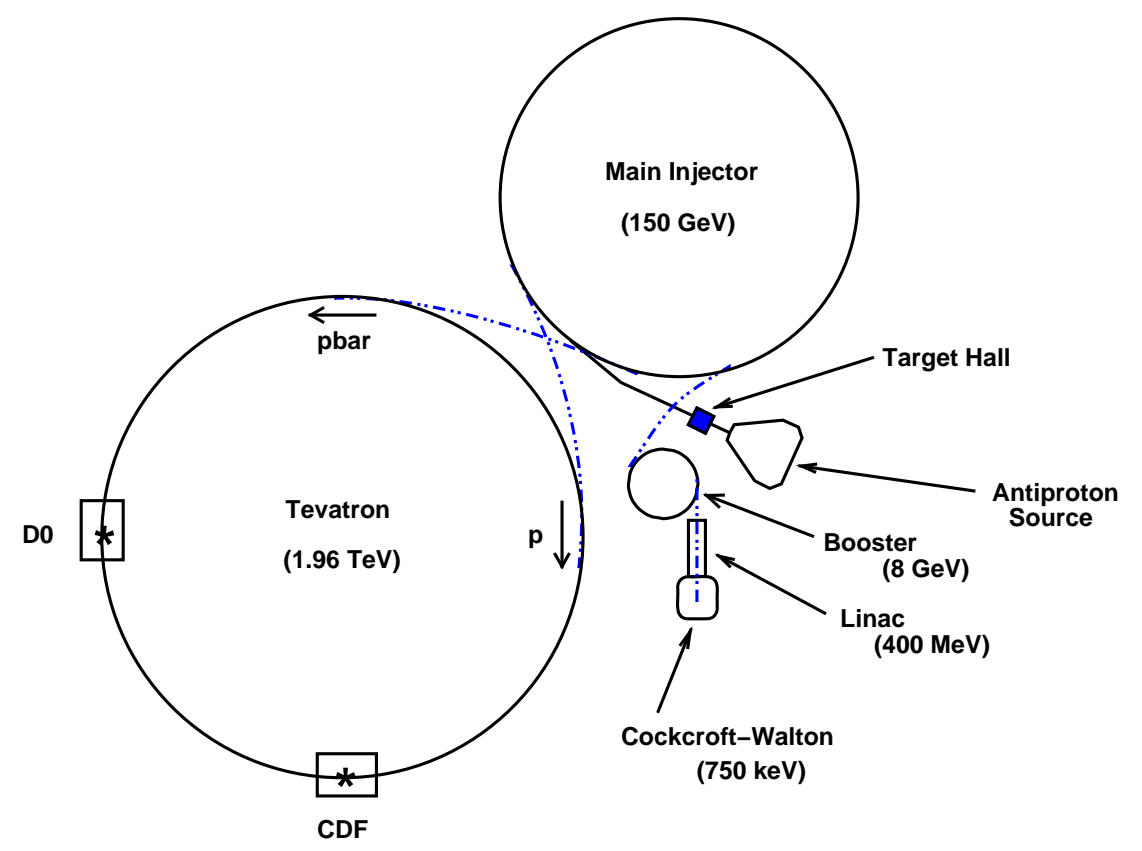

Figure 2.2: Schematic view of the accelerator complex at the Fermilab.

\subsubsection{Proton source}

The origin of the proton beam is a negative ion hydrogen source. Electrons are added to hydrogen atoms by collision with the cathode of a magnetron, thereby creating hydrogen ions which are electro-statically accelerated to $750 \mathrm{keV}$ in a Cockcroft-Walton pre-accelerator and fed into a linear accelerator, the Linac. The Linac accelerates the ions to an energy of $400 \mathrm{MeV}$. Upon injection into the next accelerator the $\mathrm{H}^{-}$ions pass a stripper foil to strip off all their electrons. This accelerator, the Booster, is the first synchrotron in the chain. It has a 
circumference of $475 \mathrm{~m}$ and accelerates the protons to $8 \mathrm{GeV}$ in 0.033 seconds. From here they are passed on to the main injector, a $150 \mathrm{GeV}$ synchrotron. Its operation modes include accelerating protons and anti-protons from $8 \mathrm{GeV}$ to $150 \mathrm{GeV}$, coalescing several proton bunches into a more intense single bunch, and providing bunches of up to $5 \times 10^{12}$ protons for the anti-proton production. The anti-proton production is described in the next subsection.

\subsubsection{Anti-proton source}

The anti-proton is created by injecting the proton beam onto anti-proton production target. Figure 2.3 shows a layout of the anti-proton source. Every 1.47 seconds the main injector provides 498 bunches which contain about $3 \times 10^{13}$ protons in total with an energy of $120 \mathrm{GeV}$. These protons are directed onto the $8 \mathrm{~cm}$ long nickel target. A lithium lens focuses secondary particles with approximately $8 \mathrm{GeV}$ that emerge from these collisions. A pulsed dipole magnet then deflects the anti-protons toward the debuncher while unwanted particles with the wrong charge-to-mass ratio are filtered out of the beam.

The selected anti-protons still have a energy spread difficult to accept for downstream accelerators. Their time spread is however small due to the fact that the protons used for their creation were grouped into bunches with narrow time spread. In the debuncher ring, an $8 \mathrm{GeV}$ synchrotron with $500 \mathrm{~m}$ circumference, this narrow time spread is transformed into a narrow energy spread. More energetic particles travel at the outside of the debuncher ring and will arrive at the radio frequency cavities later than less energetic particles which travel at the inside. Thus the particles see different phases, more energetic anti-protons are decelerated while less energetic ones are accelerated. This results, after a sufficient number of cycles, in a larger time spread but also the desired narrow energy spread. The accumulator, also an $8 \mathrm{GeV}$ synchrotron located within the debuncher ring with a circumference of $475 \mathrm{~m}$, is used to accumulate large numbers of anti-protons using further stochastic cooling. Once the beam is cooled and stacked, it is transferred to the main injector, where both protons and anti-protons are accelerated to $150 \mathrm{GeV}$ and then passed into the final accelerator, the Tevatron.

\subsubsection{Tevatron}

The Tevatron is a $p \bar{p}$ collider of approximately $6 \mathrm{~km}$ circumference. Having the opposite charge the protons and anti-protons can be accelerated within the same beam pipe. The proton and anti-proton beams are accelerated to an energy of $980 \mathrm{GeV}$. Superconducting magnets with a field strength of 4.2 Teslas keep the particles on their circular path. There are 36 bunches of protons and 


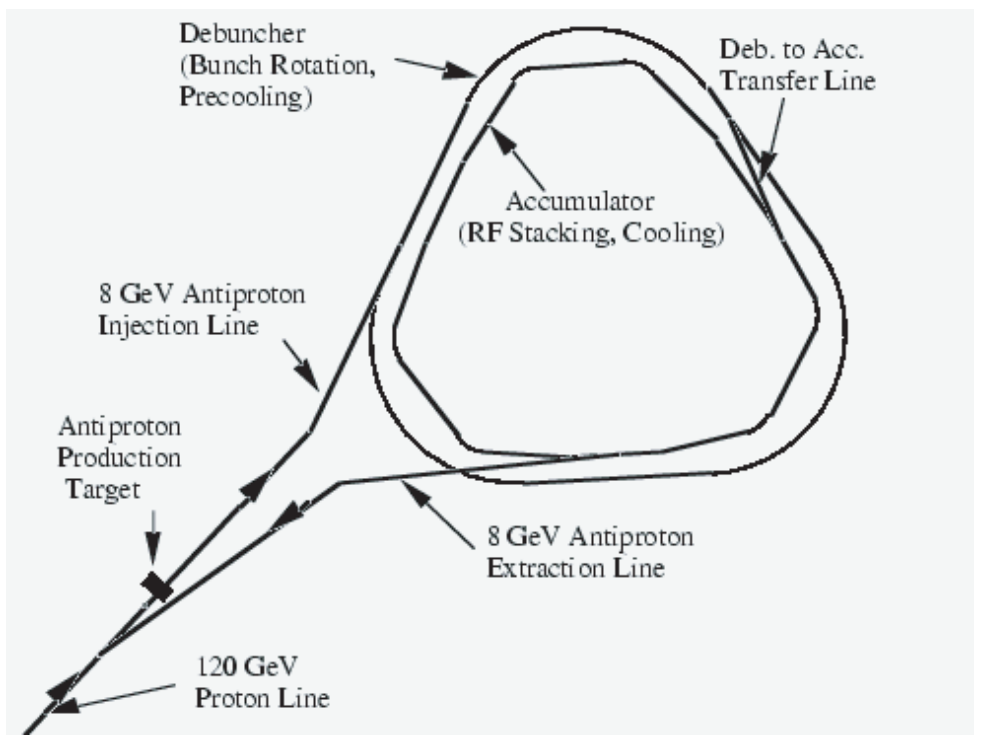

Figure 2.3: Layout of the anti-proton source.

anti-protons each in the ring. It results in a bunch spacing of 396 ns. Once the bunches reach their final energy, they are focused to collide. Currently there are two collision points on the Tevatron. The CDF detector is placed on one of these points. Other collision point is occupied by the $\mathrm{D} \emptyset$ detector.

The performance of an accelerator is generally described by a quantity, so-called luminosity $\mathcal{L}$. An event rate of the specific interaction $d \mathcal{N} / d t$ is proportional to the luminosity, shown as follows.

$$
d \mathcal{N} / d t=\mathcal{L} \cdot \sigma
$$

where $\sigma$ is the cross section of the specific interaction. For the case of a $p \bar{p}$ collider with $n$ bunches per direction, $N_{p}$ protons and $N_{\bar{p}}$ anti-protons per bunch respectively, circulating frequency $f$, the luminosity is given by

$$
\mathcal{L}=f \frac{n N_{p} N_{\bar{p}}}{4 \pi \sigma_{x} \sigma_{y}}
$$

where $\sigma_{x}$ and $\sigma_{y}$ characterize the Gaussian transverse beam profiles in the horizontal and vertical directions. The current peak instantaneous luminosity is about $1 \times 10^{32} \mathrm{~cm}^{-2} \mathrm{~s}^{-1}$, and further luminosity upgrade is ongoing [43]. 


\subsection{The Collider Detector at Fermilab}

The CDF detector is a general purpose detector dedicated to the study of physics at high-energy $p \bar{p}$ collisions at the Tevatron. Figure 2.4 shows a solid cutaway view of the CDF detector. Cross sections of the detector is also shown in Figure 2.5. In the CDF detector, a superconducting solenoid produces about 1.41 Teslas magnetic field inside which tracking detectors are contained for measurement of charged particle momenta. Calorimeter systems surround the solenoid to measure the energy of the incident particles. Outside of the calorimeter systems, muon detectors are located to detect muons which pass through the calorimeter.

The CDF detector is described in detail elsewhere [44][45]. In this section major components of the CDF detector are briefly described. Emphasis is on the detector components which are used for the $B$ meson lifetime measurement.

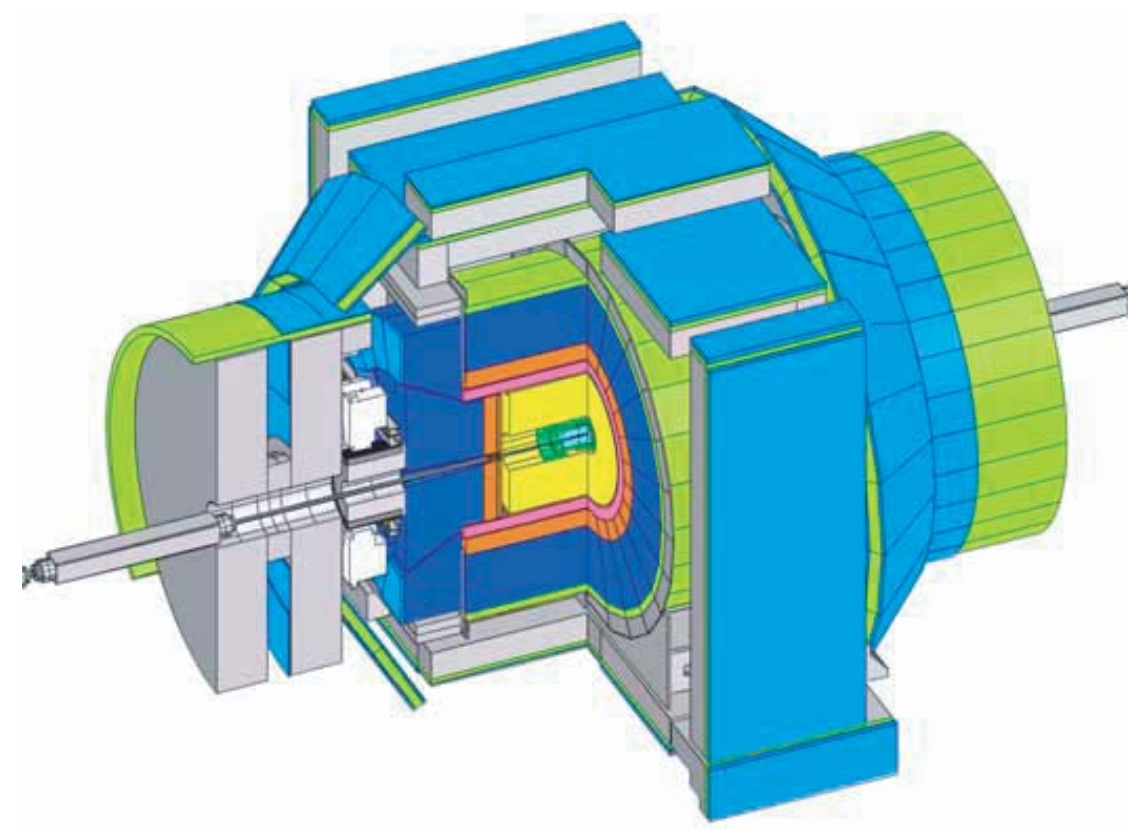

Figure 2.4: Solid cutaway view of the CDF detector. 

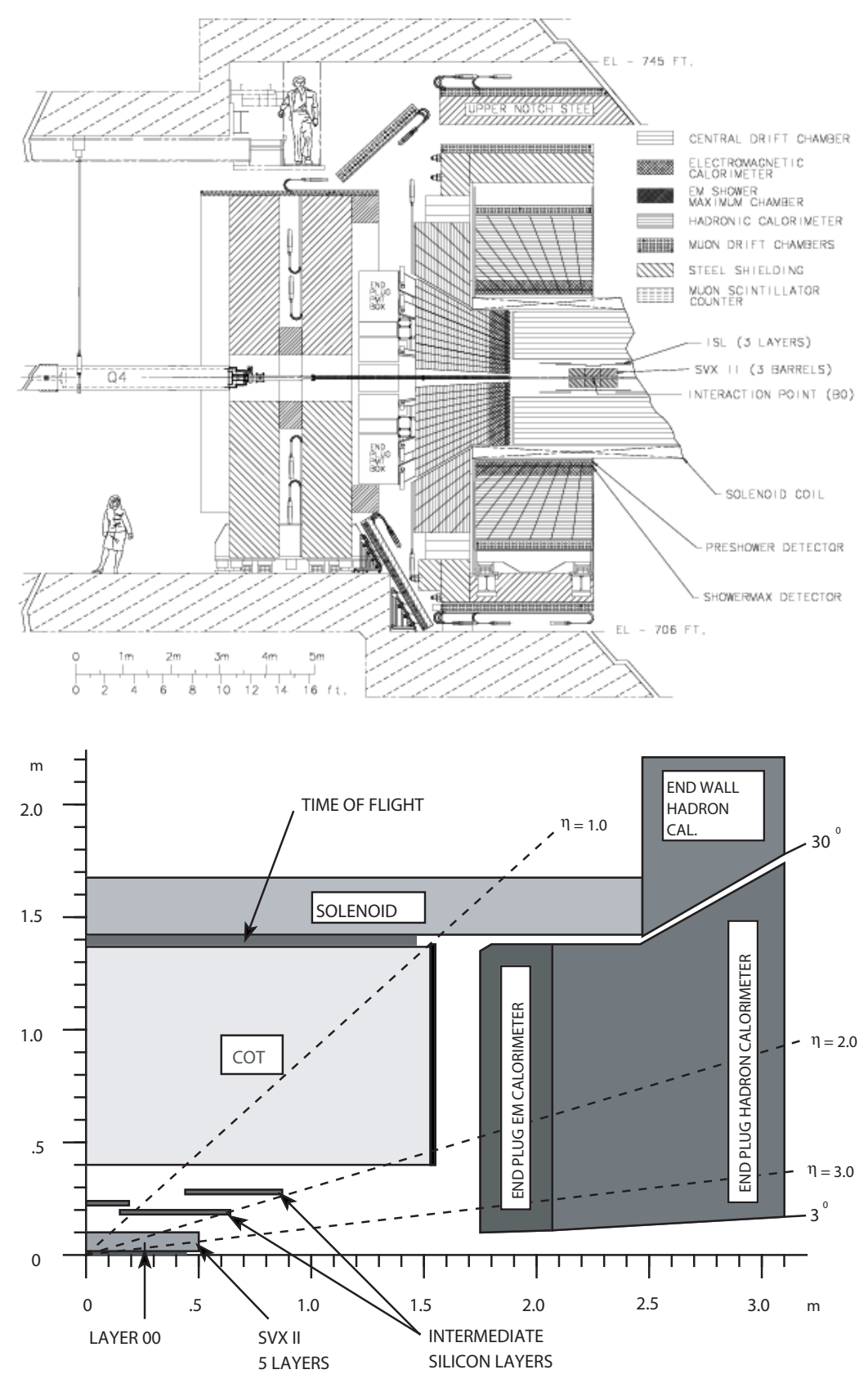

Figure 2.5: Cut views of the CDF detector. Upper is an elevation view of the one half of the whole detector, lower is for detailed view for the quarter of the detector. 


\subsubsection{Coordinate system}

The CDF coordinate system is a right-handed system with the origin taken at the center of the detector. The $z$ direction is taken along the proton beam, West to East, the y axis points vertically upward, and the $x$ axis points radially outward from the Tevatron ring. The polar angle $\theta$ and the azimuthal angle $\phi$ are defined as follows: $\theta$ is measured with respect to the positive $z$-axis and $\phi=0$ is along the positive $x$-axis with $\phi$ increasing toward the positive $y$-axis. The polar angle is often expressed in terms of the pseudo-rapidity $\eta$ defined by

$$
\eta=-\ln \left(\tan \frac{\theta}{2}\right)
$$

The transverse momentum $\left(p_{T}\right)$ and energy $\left(E_{T}\right)$ are defined as

$$
\begin{gathered}
p_{T}=p \sin \theta \\
E_{T}=E \sin \theta
\end{gathered}
$$

Figure 2.6 shows a schematic picture of the CDF coordinate system.

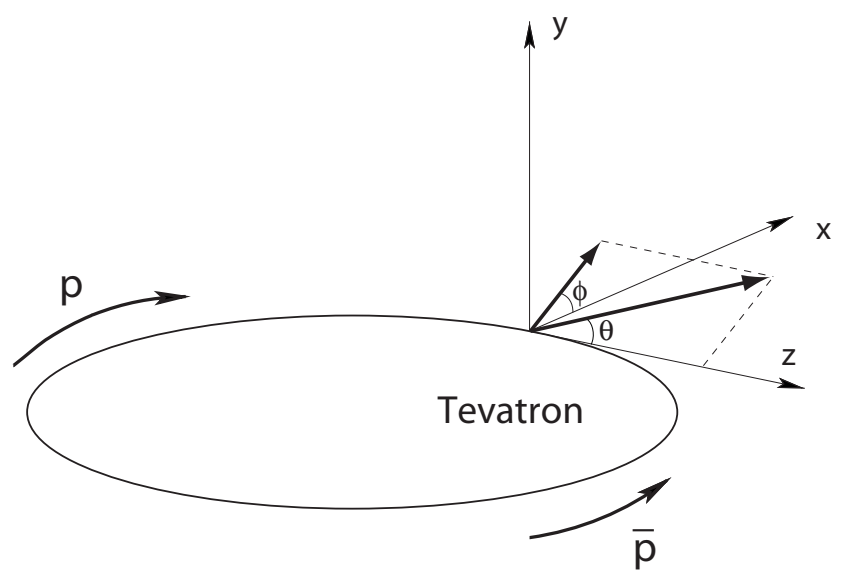

Figure 2.6: Schematic picture of the CDF coordinate system.

\subsubsection{Tracking detectors}

The efficient and precise determination of charged particle tracks is of the utmost importance to the CDF physics program. The tracking devices form the innermost part of the detector. They are located within a solenoid magnet of 
$1.5 \mathrm{~m}$ radius and $4.8 \mathrm{~m}$ length which provides a magnetic field of approximately 1.41 Teslas to bend the path of charged particles for momentum measurements.

The tracking system is composed of silicon detectors and a large open cell drift chamber consisting of 2520 drift cells.

\section{Silicon detector}

The silicon detector system comprises three concentric sub-detectors covering a region of $|\eta|<2$ and radii of $1.35 \mathrm{~cm}$ to $29 \mathrm{~cm}$ from the beam-line [47]. Figure 2.8 shows a cross section of the silicon detector system.

A charged particle produced at the primary interaction point (P.V.) first leaves a signal in the innermost silicon layer called Layer 00 (L00) which is attached directly to the beam pipe. The L00 sensors are divided into 12 wedges in $\phi$, and mounted on carbon fiber structure which is supported by the beam pipe.

The next system is the Silicon Vertex Detector (SVX II). It comprises three cylindrical barrels along the $z$ direction, each of which has an active length of $29 \mathrm{~cm}$. Each barrel consists of 12 wedges in $\phi$ with five layers of double sided AC coupled silicon micro-strip sensors, spanning in a radial range from $2.5 \mathrm{~cm}$ for the first layer (layer 0) to $10.6 \mathrm{~cm}$ for the fifth layer (layer 4 ). The $r$ - $\phi$ view of the SVX II is shown in Figure 2.7. One side of each layer provides an $r-\phi$ measurement, while the strips on the other side are oriented at $90^{\circ}$ with respect to the $z$-axis for layers 0,1 , and 3 (referred to as $r$ - $z$ measurement) and at $1.2^{\circ}$ for layers 2 and 4 (referred to as small angle stereo measurement or SAS) thus enabling three dimensional reconstruction of secondary vertices of long lived particles. The structure of SVX II with its three barrels is shown in Figure 2.7. The beryllium bulkheads on which the ladders of the three barrels are mounted carry the water cooling channels for the readout chips [46].

The outermost silicon detector is called ISL for Intermediate Silicon Layer. It closes the spatial gap between SVX II and the central drift chamber, and aids in combining the information from the two. It consists of a single layer of silicon in the central region $|\eta|<1.1$ at a radius of $22 \mathrm{~cm}$ and two layers each at $r=20 \mathrm{~cm}$ and $r=28 \mathrm{~cm}$ covering the rapidity regions $1 \leq|\eta| \leq 2$. The sensors are double sided silicon sensors. One side provides $r$ - $\phi$ measurements via axial microstrips, the other side supplies $z$ information via stereo strips (placed at $1.2^{\circ}$ for all layers). Each half ladder is made from three crystals which are microbonded to each other end to end. The ladders are supported by a carbon fiber space frame with beryllium cooling ledges. The half ladders are combined into a full ladder with readout modules at either end of this ladder. The readout hybrids are mounted off the silicon and glued onto the edge of the carbon fiber support. Figure 2.9 left picture shows a model of the ISL detector, and right one shows one of its half ladders. 

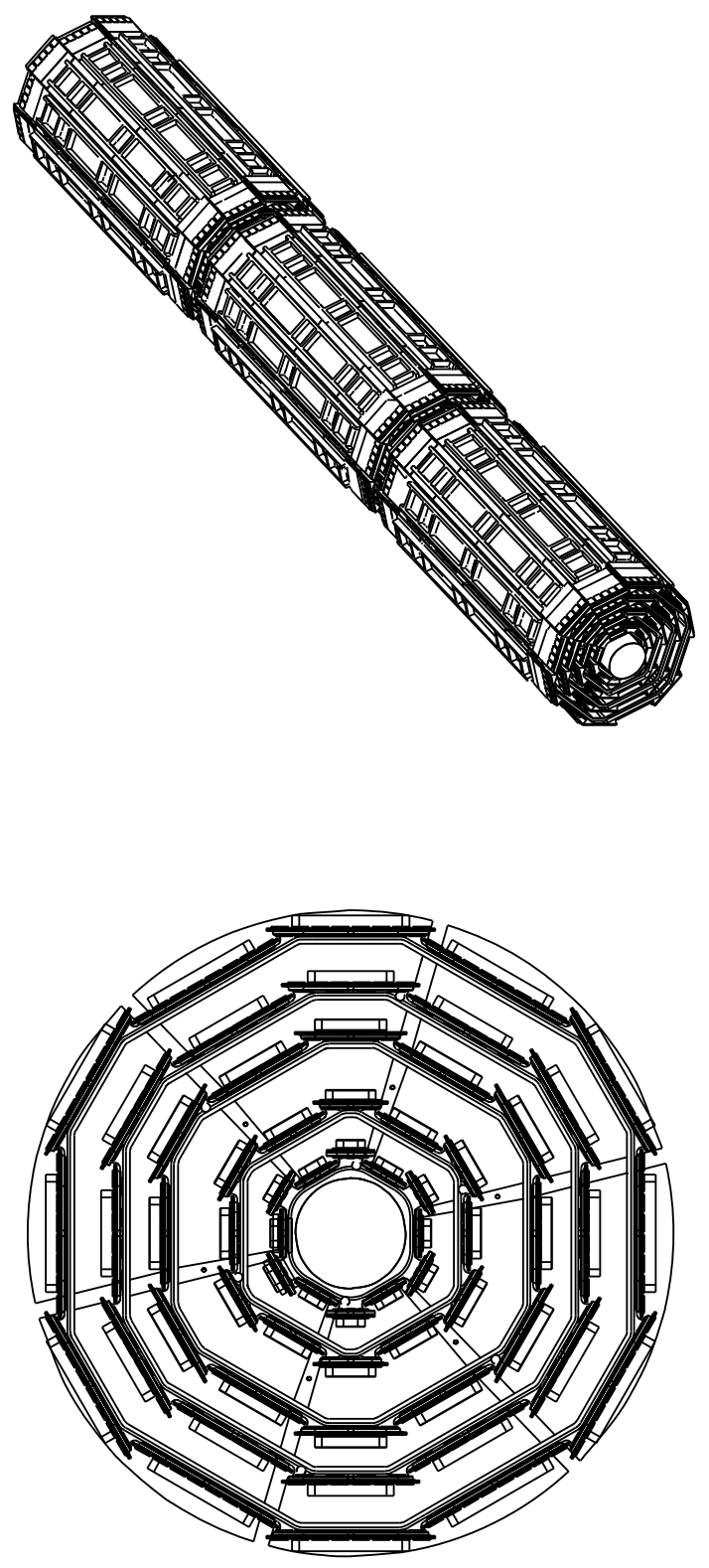

Figure 2.7: Figures of the SVX II detector. Upper figure shows a view of three barrels, and lower figure is $r$ - $\phi$ view for one barrel. Each barrel has 5 layers, and each layer has 12 wedges. 


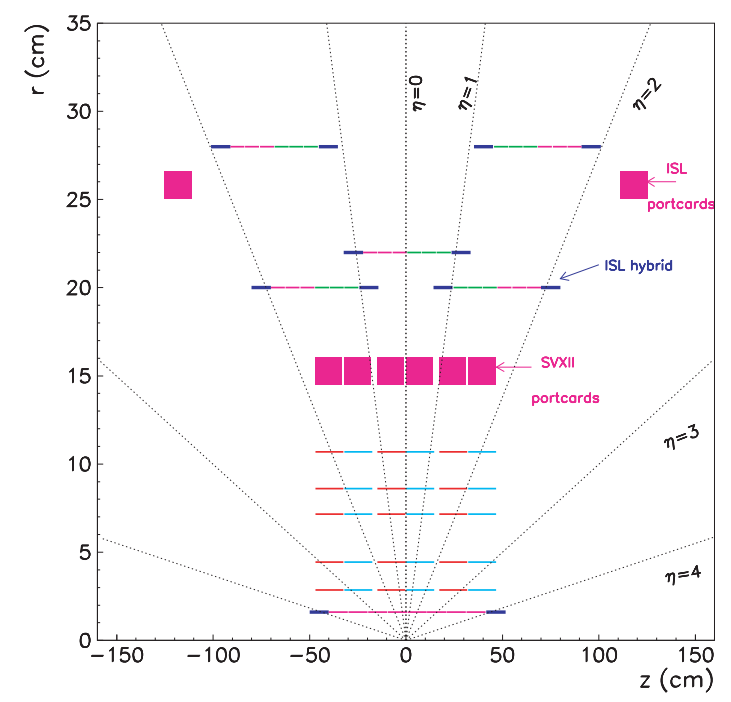

Figure 2.8: Schematic layout of the silicon tracking system.

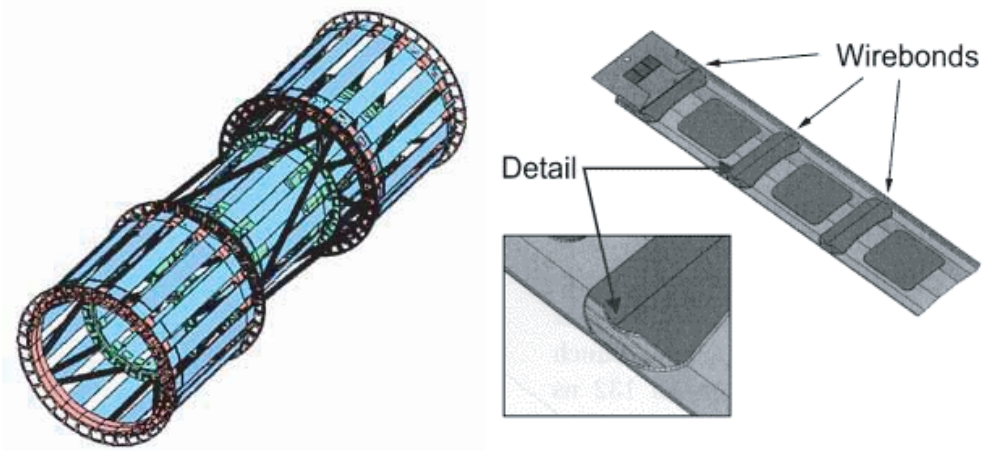

Figure 2.9: Figure of the ISL detector. 


\section{Central Drift Chamber}

The Central Outer Tracker is a large cylindrical open cell drift chamber. The chamber covers the pseudo-rapidity range $-1<|\eta|<1$ with a length of $310 \mathrm{~cm}$ of active volume. It covers the full $360^{\circ}$ in $\phi$. An mixture of argon/ethane with $50: 50$ fraction is used. The radial region from $r=43.4 \mathrm{~cm}$ to $132.3 \mathrm{~cm}$ is covered by 96 sense wires which are grouped into eight superlayers as indicated in Figure 2.10 upper picture. The superlayers arranged concentrically around the beam-line are divided into a set of drift cells. Each drift cell contains 13 potential wires interspaced by the 12 sense wires. All wires are gold plated tungsten with a diameter of $40 \mu \mathrm{m}$. The wire planes are sandwiched between gold coated $6.35 \mu \mathrm{m}$ thick Mylar plates which complete the field region. Each plane is shared by adjacent cells. Stainless steel wires are epoxied along the plane edges. The wires are attached in a parabolic shape to keep the planes flat under tension. The ends of the cells are closed electro-statically and mechanically by Mylar strips with field shaping wires attached to them. Figure 2.10 lower picture shows three drift cells. To ensure short drift distances the cells are tilted by $35^{\circ}$, which is the Lorentz angle in the COT. Axial superlayers alternate with stereo superlayers (the wires are here tilted at $\pm 2^{\circ}$ with respect to the axial direction) to provide $r-\phi$ as well as $r$ - $z$ measurements. In total the chamber consists of 30240 sense wires. The charge collected at these wires can be turned into information about the energy loss per unit length, thus providing important means of particle identification. Readout electronics as well as high voltage distribution circuits are mounted on the chamber face. On one side high voltage motherboards each for a pair of wire planes are plugged. Motherboards for FE chips (amplification, signal shaping and discrimination) are connected to pairs of wire planes on the other face. Signals are then connected to pipelined time to digital converters (TDCs) from which the chamber signals are read out, providing information available for the first trigger level. The transverse momentum resolution achieved with the COT is measured using cosmic ray events [61] to be,

$$
\sigma\left(p_{T}\right) / p_{T}^{2}=0.0017(\mathrm{GeV} / c)^{-1}
$$

Design parameters of the COT are summarized in Table 2.1. Table 2.2 details the single hit resolutions obtained with the various components of the CDF tracking system.

Using the hit information from the SVX II detector, an average impact parameter resolution of $34 \mu \mathrm{m}$ is achieved for muon tracks with a transverse momentum of approximately $1.5 \mathrm{GeV} / c$ [61]. After inclusion of the uncertainty on the transverse size of the beam-spot, this resolution increases to approximately $50 \mu \mathrm{m}$. 


\begin{tabular}{cc}
\hline \hline \multicolumn{2}{c}{ COT parameters } \\
\hline Radial coverage & 44 to $132 \mathrm{~cm}$ \\
Number of superlayers & 8 \\
Stereo angle (degrees) & $+3,0,-3,0,+3,0,-3,0$ \\
Layers per a superlayer & 12 \\
Drift field & $2.5 \mathrm{kV} / \mathrm{cm}$ \\
Maximum drift distance & $0.88 \mathrm{~cm}$ \\
Resolution per measurement & $180 \mu \mathrm{m}$ \\
Pseudo-rapidity coverage & $|\eta|<1.0$ \\
Number of channels & 30,240 \\
Material thickness & $0.016 \mathrm{X}_{0}$ \\
\hline \hline
\end{tabular}

Table 2.1: The COT design parameters.

\begin{tabular}{ccc}
\hline \hline & Coverage & Position Resolution \\
\hline L00 & $|\eta|<4$ & $6 \mu \mathrm{m}$ (axial) \\
SVX II & $|\eta|<2$ & $12 \mu \mathrm{m}$ (axial) \\
ISL & $|\eta|<2$ & $16 \mu \mathrm{m}$ (axial) \\
COT & $|\eta|<1$ & $180 \mu \mathrm{m}$ \\
\hline \hline
\end{tabular}

Table 2.2: Summary table of the position resolution of the various components of the CDF tracking system. 

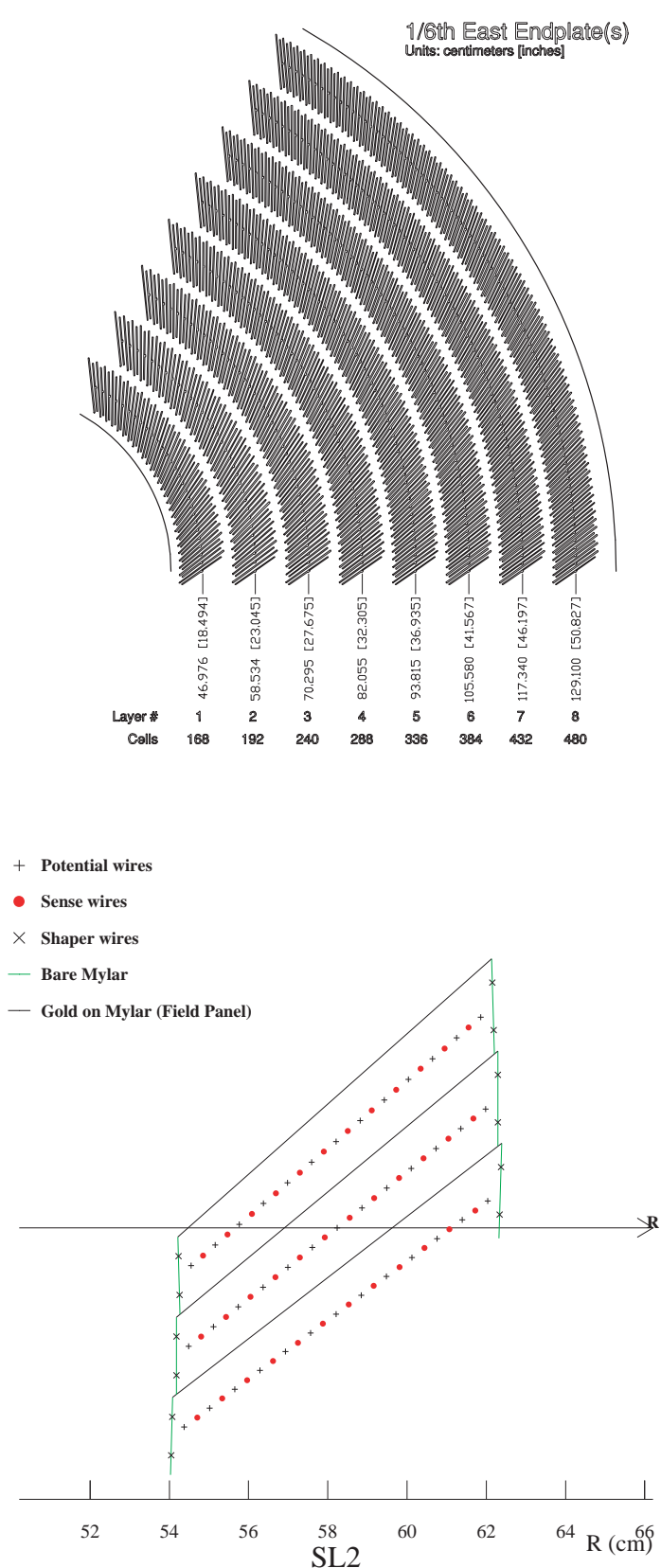

Figure 2.10: $r-\phi$ view of the COT. Upper figure is a view of east endplate. Lower figure shows three drift cells of the COT in superlayer 2. 


\subsubsection{Solenoid magnet}

The CDF detector features a 1.41 Teslas axial magnetic field throughout the tracking volume (approximately $2.8 \mathrm{~m}$ and $3.5 \mathrm{~m}$ long), which enables measurements of charge and momentum via the tracking detectors. The field points in the $-z$ direction of the CDF coordinate system. The solenoid coil is superconducting, and employs an aluminum-stabilized NbTi conductor. The normal operating field of 1.41 Teslas corresponds to a persistent current of 4,650 Amps. The conductor is cooled indirectly via liquid helium, and reinforced structurally by an external support cylinder made of aluminum. The infrastructure also includes an iron return yoke.

\subsubsection{Calorimeter}

While tracking detectors are low density position measurement devices, calorimeters are total absorption detectors designed to determine the energy of particles. Electromagnetic calorimeter is built to provide an energy measurement for electrons and photons. For energies above a few $100 \mathrm{MeV}$, the main processes that occur when these particles enter the material are bremsstrahlung for electrons and pair production for photons. These two processes lead to the development of an electromagnetic shower, which is depicted in the left part of figure 2.11. A photon entering the detector material produces an electron-positron pair with its energy distributed between the two particles. After traversing a radiation length $X_{0}$ of material the probability for the photon to produce such a pair is $54 \%$. The newly created particles then radiate new photons in the field of nuclei, a process known as bremsstrahlung. The shower ends when the energy of the particles falls below the energy necessary to create new electron-positron pairs. At this point ionization and excitation are the dominant processes. The length of the electromagnetic calorimeter is designed such that electrons and photons dissipate all their energy in the detector volume.

The CDF calorimeter is divided into central, endwall, and plug calorimeters. Since the $B$ lifetime analysis uses only the central part, here we describe only the central calorimeter. The central calorimeter extends to $|\eta|<1.1$.

\section{Central pre-radiator}

Even though the tracking detectors are designed to contain as little material as possible, the magnet coil and the cryostat of the superconducting solenoid themselves comprise already 0.86 to 1.1 radiation length of material depending on their angle with respect to the $z$-axis. This material acts as absorber, or radiator and forms, in conjunction with a single multi-wire proportional chamber (MWPC) plane, the innermost part of CDF's calorimeter system, the 


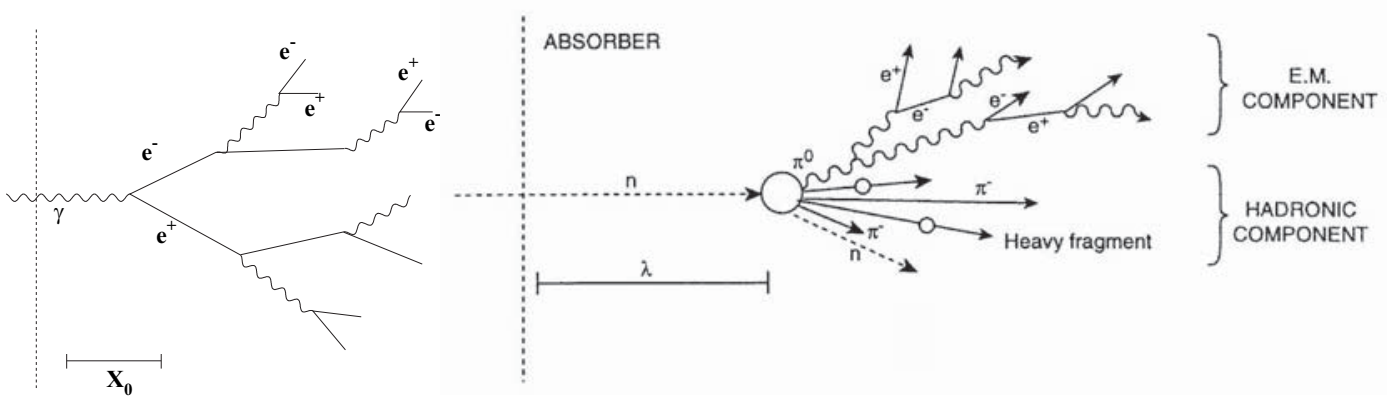

Figure 2.11: Development of an electromagnetic shower (left) and a hadronic shower (right).

Central Pre-Radiator (CPR) [48]. The MWPCs are fitted into a $5 \mathrm{~cm}$ gap between the solenoid and the central electromagnetic calorimeter. Altogether there are 96 chambers. Each chamber contains 32 sense wires and 31 field wires and is filled with $\mathrm{Ar} / \mathrm{CO}_{2}$. The system is installed at a distance of $168.29 \mathrm{~cm}$ from the beam-pipe to sample showers that are initiated before the actual electromagnetic calorimeter.

\section{Central electromagnetic calorimeter}

The Central Electromagnetic Calorimeter (CEM) [50] follows radially outward the CPR. CDF's electromagnetic and hadronic calorimeter have a common structure: they are both sampling calorimeters with alternating layers of scintillator and absorbing material. To combine the good energy resolution of a scintillator with good position measurement, the CEM is additionally built as a hybrid of a scintillator based sampling calorimeter for energy measurement and a 2D strip chamber for position determination and measurement of the longitudinal shower development. The energy resolution achieved with this design is measured to be $\sigma(E) / E=13.5 \% / \sqrt{E_{T}}$ with $E_{T}$ in $\mathrm{GeV}$. The position resolution achieved is $2 \mathrm{~mm}$ at $50 \mathrm{GeV}$. Following an inner base plate of aluminum the calorimeter consists of alternating layers of $5 \mathrm{~mm}$ thick SCSN-38 polystyrene scintillator [49] (31 layers in total) and $3.2 \mathrm{~mm}$ thick lead cladded with aluminum (30 layers). The metal comprises mainly lead with small amounts of aluminum. However, to retain constant radiation length thickness it is in places substituted with acryl. The sandwiched layers can be divided into two halves in $\eta$, each stretching for about $2.5 \mathrm{~m}$ along the $z$-axis and into modules of $15^{\circ}$ in $\phi$, with small cracks at the boundaries, resulting in 48 modules in total. Each module is subdivided into 10 units along $z$ with $\Delta \eta \sim 0.11$. The $\eta$ - $\phi$ modules 
are aligned to point back to the center of the detector. Together with the cryostat the system comprises approximately $19 X_{0}$ of material. Wavelength shifters installed on the $\phi$ surfaces guide the photons produced in the scintillators to photomultiplier tubes. Figure 2.12 shows one of the 24 wedges in $\phi$ with its light gathering system. Embedded in between the eighth layer of lead and the ninth scintillator layer, which corresponds to a depth of maximum average shower development, at approximately $6 X_{0}$ inside the CEM is the Central Electromagnetic Strip detector (CES), consisting of orthogonal strips and wires (to determine the shower position as well as the transverse shower development). The chambers are operated with an $\mathrm{Ar} / \mathrm{CO}_{2}$ mixture. To retain a constant radiation length thickness up to the CES, lead is substituted with acrylic in certain layers in high 7 towers.

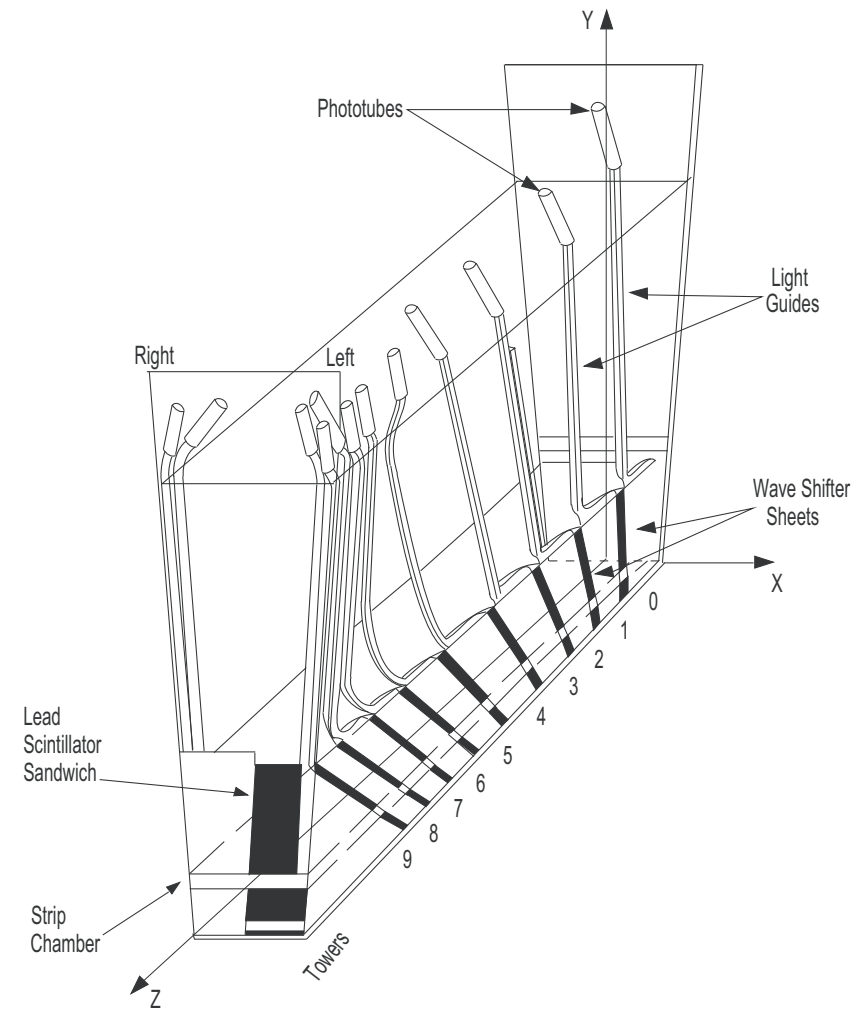

Figure 2.12: One wedge of the CEM with its light collecting system. 


\section{Central hadron calorimeter}

The hadrons have a different shower behavior and the electromagnetic calorimeter does not have much stopping power for hadrons. Thus an additional calorimeter, the Central Hadronic Calorimeter (CHA) is built radially outside the electromagnetic calorimeter to measure the energy of hadrons. If high energy hadrons enter on a block of matter, inelastic as well as elastic scattering will occur between the particles and the nucleons inside the material. Thus energy is transferred and secondary particles are produced, that further collide inelastically: A hadron shower occurs. The basic interaction responsible for the shower development is the strong interaction. In addition, the shower has an electromagnetic component originating mainly from the decay of secondary $\pi^{0}$ 's, as can be seen in the right drawing of Figure 2.11.

The CHA is a scintillator based sampling calorimeter. It extends to $|\eta|<$ 0.9 and covers apart from the slightly smaller $\eta$ region essentially the region covered by the CEM with the towers of the CHA built in blocks of $0.11 \times 15^{\circ}$ in the $\eta$ - $\phi$ plane to match the towers of the CEM. The CHA also comprises 48 modules, each of which consists of 32 layers of $10 \mathrm{~mm}$ thick scintillator, PMMA (Poly Methyl MetAcrylate) naphthalene, sandwiched between $2.5 \mathrm{~cm}$ thick iron absorber plates. It has a total thickness corresponding to $4.5 \lambda$. The energy resolution of the CHA is $\sigma(E) / E=50 \% / \sqrt{E}$ with $E_{T}$ in $\mathrm{GeV}$.

\subsubsection{Muon detector}

Four subsystems of muon chambers and scintillation counters form the outermost part of the CDF II detector. These are called CMU, CMP, CMX, and IMU, and have different solid-angle coverages. The CMU and CMP cover the central part, and the CMX and IMU provides higher $\eta$ coverage of the muon identification. Coverages of each subsystems are shown in Figure 2.13.

The calorimeter steel, the return yoke of the magnet, additional steel walls provide sufficient absorber material to stop most hadrons, leaving only muons to reach these outermost chambers due to their higher penetration power. Occasionally a hadron can enter the muon system if it either fails to interact strongly in the absorber material or if the interaction occurs too close to the muon chambers allowing secondary particles to escape the calorimeter. This is called a punch-through.

Since the $B$ meson lifetime analysis uses only the CMU and CMP, we describe only these subsystems in this subsection below. 


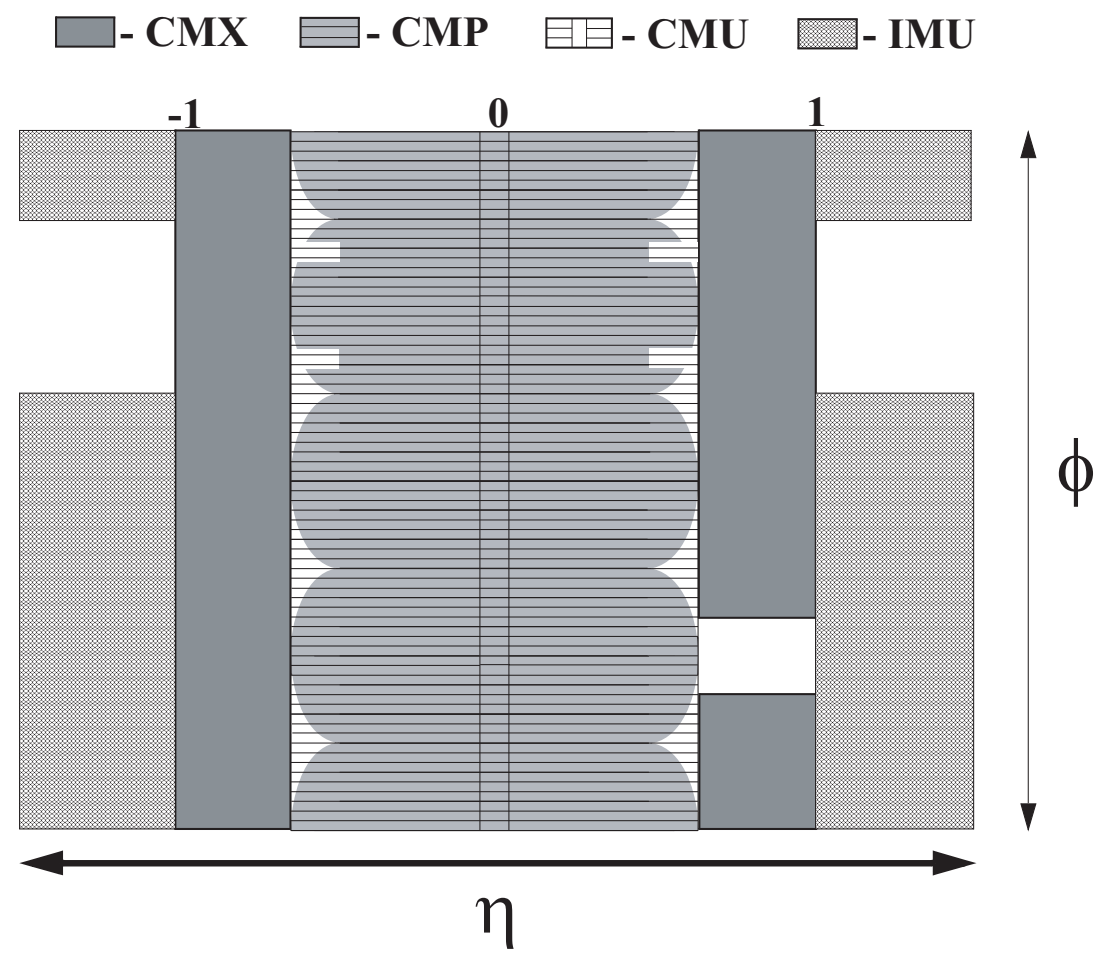

Figure 2.13: Location of the muon detectors for Run II in azimuth $\phi$ and pseudo-rapidity $\eta$.

\section{Central muon detector}

The Central MUon detector (CMU) covers $|\eta|<0.6$ region with a gap of $18 \mathrm{~cm}$ between the east and west chambers at $\eta=0$, as shown in Figure 2.13. The muon system is situated behind approximately $5.5 \lambda$ of absorber from the calorimeter which results in a $p_{T}$ threshold for muon detection of $1.4 \mathrm{GeV} / c$. It consists of a total of 2304 single wire drift cells grouped into 144 modules. Figure 2.14 left drawing shows the position of the CMU detector in $r$ - $\phi$ and $r-z$ projections with three modules matching a calorimeter tower of $15^{\circ}$. The instrumented region in a wedge spans $12.61^{\circ}$. A cross section of one such module with its drift tube cells is shown in Figure 2.14 right drawing. These cells are of dimension $2.68 \times 6.35 \times 226 \mathrm{~cm}$ with a $50 \mu \mathrm{m}$ stainless steel wire in the center. The wire is held at $2.325 \mathrm{kV}$. The side walls of each cell are held at $-2.500 \mathrm{kV}$ and top and bottom walls at ground in order to construct a uniform drift field.

As Figure 2.14 shows, alternating chambers are shifted slightly with respect to each other so that the combination of two alternating cells projects back to 
the interaction point while left-right ambiguity is resolved. Comparing the drift time of two such cells allows a crude measurement of the track's momentum and can thus be used for triggering. Adjacent wires are grouped together for readout.
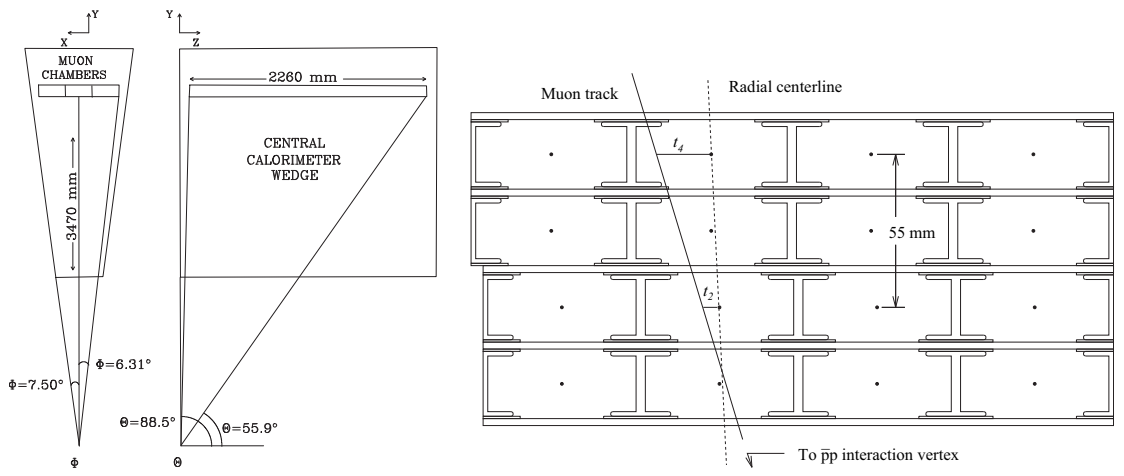

Figure 2.14: Drawings of the CMU. In right drawing locations in $r-\phi$ and $r-z$ are shown for three modules. Left drawing is a cross section of a CMU module showing its 16 drift tube cells.

\section{Central muon upgrade}

The Central Muon uPgrade (CMP) is installed after an additional $60 \mathrm{~cm}$ steel radially outward from the $\mathrm{CMU}$ resulting in about 7.8 interaction lengths for pions. There are 1064 single wire drift cells with four layers glued together to form 266 four layer stacks. The cells in a stack are staggered with respect to each other with a half cell spacing. Unlike most other detectors the CMP is not cylindrically shaped but forms a large rectangle around the CMU with a slightly complicated structure at top and bottom. This square structure is shown in Figure 2.15. This geometry, with a fixed length in $z$ results in $\phi$ dependent dips in the $\eta$ coverage which is visible in Figure 2.13. The drift cells are $2.5 \times 15 \mathrm{~cm}$ and about $640 \mathrm{~cm}$ long (some are shorter due to space restrictions). They are single wire drift cells operated in proportional mode. The gold-plated tungsten wire is held at $+5.6 \mathrm{kV} .17$ cathode strips at the top and bottom of the cell shape the field in the cell. The central strips are held at $3 \mathrm{kV}$ with the voltages of the strips at either side subsequently lowered by $375 \mathrm{~V}$ until they reach ground potential at the cell walls. Unlike the CMU, the CMP provides no $z$ information. The TDCs provide trigger information from coincidences of nearby wires in association with information from the CMU. A layer of 216 scintillation counters (CSP) surrounds the CMP to provide timing 
information. The counters measure $2.5 \times 15 \times 320 \mathrm{~cm}$ and are read out by single phototubes.

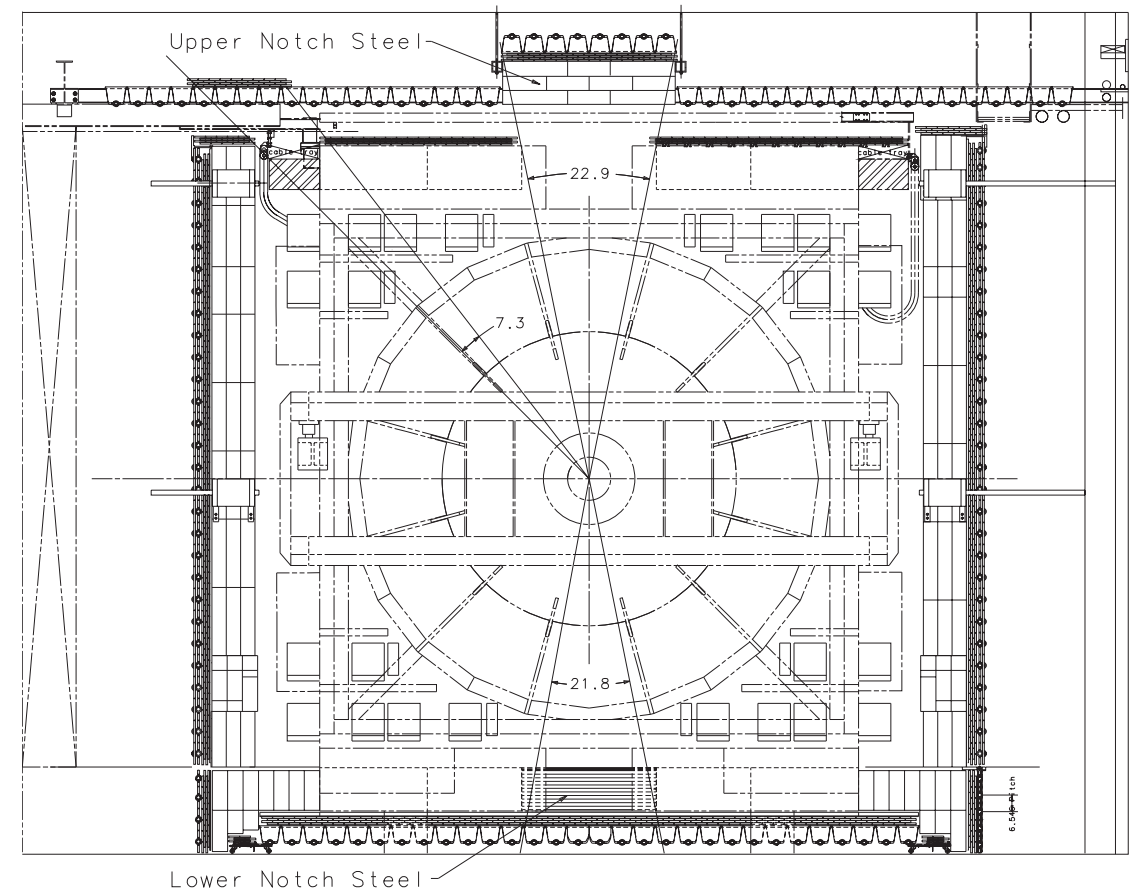

Figure 2.15: Configuration of the CMP. The dark gray square structure surrounding the central detector shows the CMP modules and scintillators.

\subsubsection{Trigger system}

A well designed trigger system is crucial for hadron collider experiments which have an event rate much higher than the ability of the event recording. At the CDF experiment the event rate is effectively equal to the inverse of the bunch crossing time, resulting in 2.5 million events per second for the current bunch crossing time of $396 \mathrm{~ns}$. However at most 50 of those 2.5 million events can be recorded by logging system. The purpose of the trigger is to select efficiently the most interesting events for further analysis whilst achieving the necessary reduction in the event rate. Here the definition of an interesting event depends on the specific physics goal, and multiple trigger paths are used to accommodate different needs.

The CDF have three level trigger system. Figure 2.16 shows a diagram of the data flow through the three-level trigger system. The first two levels 
comprise buffered and pipelined hardware triggers while the third level consists of an offline processing farm. Each level provides a sufficient rate reduction for processing in the next level with minimal dead time.

Figure 2.17 is a more detailed chart showing which detector components and trigger subsystems contribute to the global trigger decision at Level 1 (L1) and Level 2 (L2). Level 1 is a synchronous trigger, processing information from the calorimeters, the muon chambers and the COT simultaneously. It is fully pipelined with each detector element capable of buffering information from 42 events locally. As shown in the block diagram of Figure 2.17 the Level 1 trigger decision is based on a combination of information from the calorimeter, the central tracking chamber COT and the muon system. The XFT (eXtremely Fast Tracker) reconstructs tracks within $2.7 \mu$ s based on the COT hit information. The reconstructed tracks are then passed to the XTRP (track eXTRaPolator), which selects tracks above $1.5 \mathrm{GeV} / c$, extrapolates those tracks to the calorimeter and to the muon chambers and distributes this information or the tracks themselves to the L1 and L2 subsystems. The L1 calorimeter trigger (L1CAL) can then form calorimeter objects such as electrons, jets and photons, based on whether a calorimeter cluster has a matching track. The L1 muon trigger (L1MUON) similarly forms muon objects, while the L1 track trigger (L1TRACK) is based on information from the XFT. An observation of more than four tracks generates an auto accept of the event. Additionally the $p_{T}$ and $\phi$ information from tracks can be used for a trigger acceptance decision. The global Level 1 trigger then accepts or rejects an event based on the number and type of objects obtained from L1CAL, L1TRACK and L1MUON.

Upon a Level 1 accept all information is passed to the Level 2 subsystems. To allow an input rate of approximately $50 \mathrm{kHz}$, Level 2 has a buffer for four events and is pipelined, splitting the decision process into two separate stages, each of which takes about $10 \mu \mathrm{s}$. This yields a latency of $20 \mu \mathrm{s}$ but the effective time between two decisions is now $10 \mu$ s thus reducing the dead time. In the first stage the event is built using information from the shower maximum detector (XCES) and the L1 triggers, XFT and L1MUON. Simultaneously the second level calorimeter trigger L2CAL yields improved cluster finding and the SVT track processor finds tracks in the SVX II using hits from the $r$ - $\phi$ strips of the SVX II and tracks from the XFT. This collection of event data in the memory of the Level 2 processors takes about $10 \mu \mathrm{s}$ after which the event is examined in stage two using alpha processors to make a trigger decision. The event rate at Level 2 is reduced using the advanced jet cluster finding from L2CAL and the improved electron and photon identification achieved with the CES, which has better spatial resolution than the calorimeter itself.

Upon a Level 2 accept, the full detector information collected from the front end electronics is read out by the data acquisition system and sent to Level 
3 [51], a farm of processors. Here the entire event is built and sophisticated trigger decisions, based for instance on full 3D track reconstruction, are made. Upon a Level 3 accept the event is written to mass storage.

\subsection{7 $8 \mathrm{GeV}$ single lepton triggers}

The trigger used for this $B$ lifetime analysis is the $8 \mathrm{GeV}$ single muon and electron triggers. The requirements for these triggers are described below.

Muon trigger :

- Level 1:

- Muon have stubs on CMU and CMP

- $p_{T}>4 \mathrm{GeV} / c$

- Level 2 :

$-p_{T}>8 \mathrm{GeV} / c$

- Level 3 :

$$
\begin{aligned}
& -p_{T}>8 \mathrm{GeV} / c \\
& -|\Delta x(\mathrm{CMU})|<15 \mathrm{~cm} \\
& -|\Delta x(\mathrm{CMP})|<20 \mathrm{~cm}
\end{aligned}
$$

Electron trigger :

- Level 1 :

$$
\begin{aligned}
& -p_{T}>4 \mathrm{GeV} / c \\
& -E_{T}>4 \mathrm{GeV} \\
& - \text { EM cluster is in the CEM } \\
& -E_{\mathrm{HAD}} / E_{\mathrm{EM}}<0.125
\end{aligned}
$$

- Level 2 :

- prescaling

- Level 3 :

$$
\begin{aligned}
& -p_{T}>8 \mathrm{GeV} / c \\
& -E_{T}>8 \mathrm{GeV} \\
& -\mathrm{LSHR}<0.2
\end{aligned}
$$




$$
\begin{aligned}
& -|\Delta x|(\mathrm{CES})<3 \mathrm{~cm} \\
& -|\Delta z|(\mathrm{CES})<5 \mathrm{~cm} \\
& -E_{\mathrm{HAD}} / E_{\mathrm{EM}}<0.055
\end{aligned}
$$

The parameters used in Level 1 and 2 are determined by Level $1 /$ Level 2 hardware, while Level 3 parameters are optimized from offline study. The parameters for muon and electron identification used offline are explained in sections 3.2 and 3.3 . 


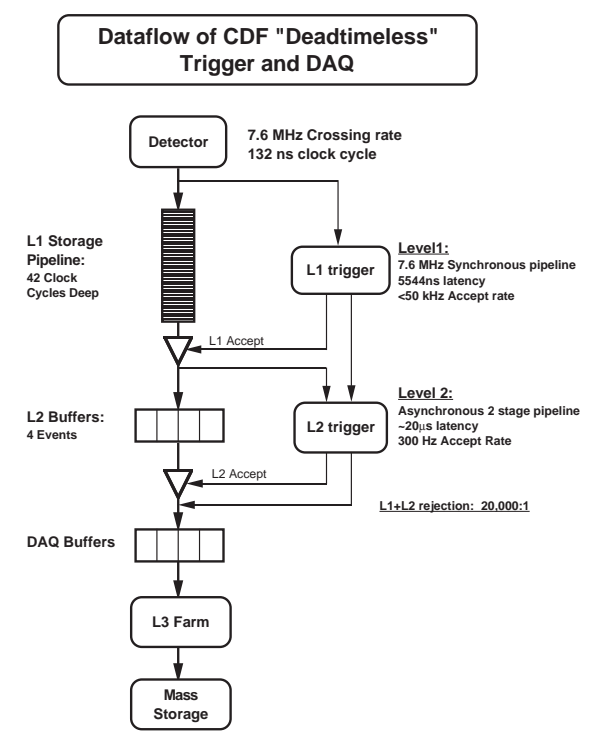

Figure 2.16: Architecture of the three-level pipelined and buffered trigger system used in the CDF experiment. (The numbers are for 132 ns operation, while actual operation is with 396 ns bunch spacing.)

\section{RUN II TRIGGER SYSTEM}

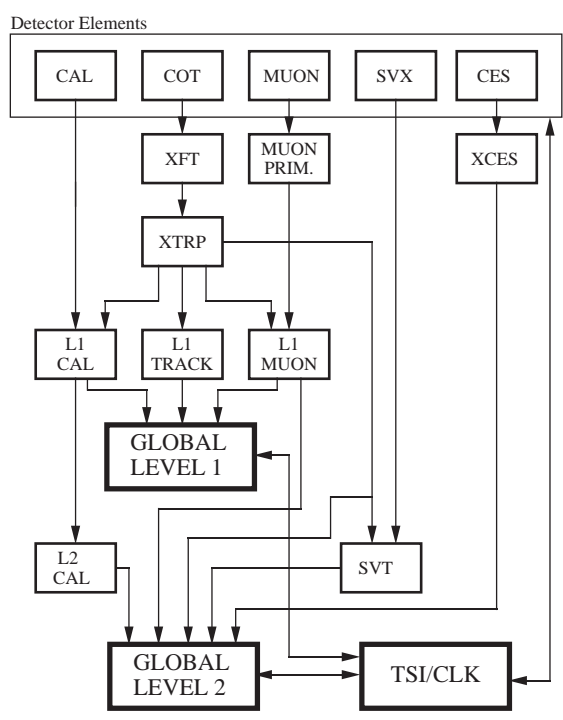

Figure 2.17: Block chart of the trigger system for CDF experiment. The trigger Supervisor Interface (TSI) distributes signals between the trigger and the data acquisition system such as the bunch crossing signal and Level 1 and Level 2 accept or reject signals. 


\section{Chapter 3}

\section{Event reconstruction}

In this chapter, reconstruction of the $B$ mesons is described. Since we can not fully reconstruct the $B$ signal with the semileptonic decay, we utilize the lepton + charm meson combination to identify the $B$ signal. From the lepton + charm pair, we reconstruct the semileptonic $B$ decay vertex and measure the decay length.

\subsection{Tracking}

At first we reconstruct tracks of charged particles using COT and silicon detector hit information [52]. The track reconstruction starts with hits left by a particle in the central tracking chamber. The ionization caused by charged particles passing through the COT volume is collected by its sense wires and the COT hardware which provides a measurement of the drift time for a detected hit. Then the hit position is calculated from the wire position, the drift time and the drift velocity. At this stage there is an ambiguity as the drift time measurement of a single hit does not determine which side of the sense wire the track passed. This is an issue resolved during track finding.

Once all COT hit candidates in the event are known, the eight superlayers are scanned for line segments, defined as triplets of unused aligned hits which belong to consecutive layers in a superlayer. A list of candidate segments is then formed and ordered by increasing slope of the segment with respect to the radial direction and the hit positions of a segment are fitted to a straight line. The fitted line segments are linked by track finding algorithm and a track is formed.

Analogously to finding hits in the COT through ionization collected by the sense wires, hits are formed from the charge collected on the silicon strips. To utilize the silicon information for the tracking, the COT tracks that are already found are used as seeds of the silicon hits finding. In a certain distance 
to the COT seed track (called "road" of the seed track), unused silicon hits are searched. If the silicon hits are found, again the track linking is performed including the silicon hits information found in the road.

Eventually a reconstructed track can be parameterized by five parameters which are chosen to be:

- $d_{0} \ldots$ the track impact parameter, namely closest distance between the collision point and the track.

- $z_{0}$ and $\phi_{0} \ldots$ the $z$ and $\phi$ position of the track at the point of closest approach to the $z$-axis.

- $\lambda=\cot \theta \ldots \theta$ is the polar angle in the CDF coordinate system.

- $c=1 / 2 r \ldots$ the half-curvature of the track

Any other track parameters (e.g. $p_{T}, \eta \ldots$ ) are calculated from these five parameters. More detailed description on the tracking can be found elsewhere [53][54].

\subsection{Muon identification}

Muons are selected using track and muon detector information. As shown in Figure 3.1, muons leave a track in the tracking system. After passing through the calorimeter and steel walls. They leave hit information on the muon detectors. From the hit information of the muon chambers, muon trajectory on the muon detector is reconstructed. This reconstructed trajectory, so-called "stub", is linked with track reconstructed in the tracking system and extrapolated toward the muon detector. Differences between expected and actually detected stub positions and slopes are useful to discriminate fake muons which come from punch-through hadrons. There are some parameters to represent this difference on the CMU and CMP. First one is just a distance of those two, called $\Delta x$ ( $\Delta z$ in case for distance of $z$ direction). The second is muon $\chi^{2}$. This quantity is calculated considering effect of muon multiple scattering in the materials before the muon detector. Expected scattering is calculated with Monte Carlo simulation. From the distance $\Delta x$ and magnitude of the expected scattering, $\chi^{2}$ is calculated. In this lifetime analysis we use only CMU and CMP to identify muons. CMU have sensitivity for both $x$ and $z$ hit information, besides CMP can only have $x$ direction. Thus totally six parameters (of course not all independent $), \Delta x(\mathrm{CMU}), \Delta z(\mathrm{CMU}), \Delta x(\mathrm{CMP}), \chi_{x}^{2}(\mathrm{CMU}), \chi_{z}^{2}(\mathrm{CMU})$, $\chi_{x}^{2}(\mathrm{CMP})$, are available for muon identification. The $\Delta x$ information is used at the single muon trigger. In the offline analysis we cut on $\chi^{2}$ 's for further rejection of the fake muons. 
- $\chi_{x}^{2}(\mathrm{CMU})<6, \chi_{z}^{2}(\mathrm{CMU})<5, \chi_{x}^{2}(\mathrm{CMP})<6$ for $D^{0}, D^{*+}$ sample

- $\chi_{x}^{2}(\mathrm{CMU})<7, \chi_{z}^{2}(\mathrm{CMU})<9, \chi_{x}^{2}(\mathrm{CMP})<7$ for $D_{s}^{+}$sample

Slightly looser cuts are applied for the $\ell^{-} D_{s}^{+}$selection. These numbers are found to achieve best statistical power for the $\bar{B}_{s}^{0}$ lifetime measurement. For the $\ell^{-} D^{0}$ and $\ell^{-} D^{*+}$ selection, since we have much larger number of events than the $D_{s}^{+}$case, somewhat tighter cuts are applied to increase the signal-to-noise ratio $(S / N)$.

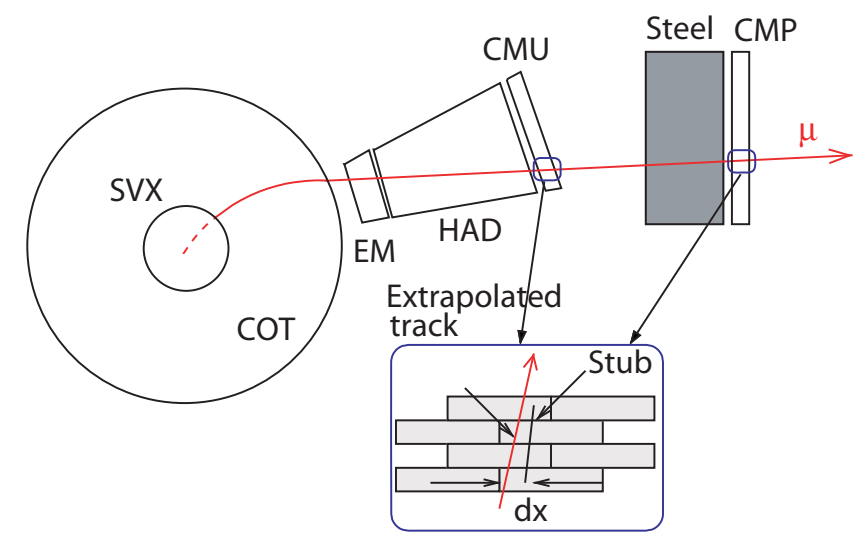

Figure 3.1: Passage of a muon through detectors.

\subsection{Electron identification}

Electron is a charged particle which leaves a track in the tracking detectors, and deposits almost of its energy in the electromagnetic calorimeter. So the electromagnetic shower shape observed in the CEM and associating track parameters can be a good handle for the electron identification.

Identification of the electron starts with CEM clustering. CEM towers with the measured energy greater than $3 \mathrm{GeV}$ are picked up as "seed" of the clustering. From a seed tower, adjacent towers which have energy deposit are linked, and the calorimeter cluster is formed. For the electromagnetic (EM) cluster, maximum cluster size is 3 towers in $\eta$ and $15^{\circ}$ (one wedge) in $\phi$ direction.

After finding a valid EM cluster, a series of other objects, associated track, shower-maximum cluster, pre-radiator cluster, are linked to the EM cluster to 
form an electron candidate. For each electron candidate, associated track is iteratively extrapolated to the plane of the CES for the wedge containing the associated EM cluster.

Following variables are used for electron identification at the trigger and offline selection. Numbers shown in parentheses are actually used for offline selection.

- associated track $p_{T}(>8 \mathrm{GeV} / c)$

- EM cluster $E_{T}(>8 \mathrm{GeV})$

- $E_{\mathrm{HAD}} / E_{\mathrm{EM}}(<0.04$ if number of associated track is 1 , or $<0.1$ if number of associated track is greater than 1)

This quantity is the ratio of the total energy in the hadron calorimeter to the total energy in the EM calorimeter for the towers included in and projection to the EM cluster.

- $\operatorname{LSHR}(<0.2)$

This variable is defined as follows.

$$
\mathrm{LSHR}=0.14 \sum_{i} \frac{E_{i}^{\text {adjacent }}-E_{i}^{\text {expected }}}{\sqrt{(0.14 \sqrt{E})^{2}+\left(\Delta E_{i}^{\text {expected }}\right)^{2}}}
$$

where the sum is over towers in the electromagnetic cluster, and $E_{i}^{\text {adjacent }}$ and $E_{i}^{\text {expected }}$ are the measured and expected energy in towers adjacent to the seed tower. The expected energy is calculated from the test beam data. $\Delta E_{i}^{\text {expected }}$ denotes an error on the energy estimation.

- CES $\Delta x$ and $\Delta z(<1.4 \mathrm{~cm}$ for $|\Delta x|,<2 \mathrm{~cm}$ for $|\Delta z \sin \theta|)$

These are differences between the extrapolated track to the CES and the actual position measured in the CES, in $x$ and $z$ directions respectively.

- $\operatorname{CES} \chi_{x}^{2}$ and $\chi_{z}^{2}(<15$ for both $)$

These quantities are calculated from comparison between expected and observed CES pulse height distributions.

- $E / p(0.75 \sim 1.4)$

This number is a ratio of the cluster energy $E$ and associated track momentum $p$.

After applying the electron ID cuts, conversion electrons are removed with the method by Gold [55] 


\subsection{Reconstruction of the $D^{0}, D^{*+}$ mesons}

In this section we describe about reconstruction of the $D^{0}$ and $D^{*+}$ mesons which are the daughter particles of the $B^{-} / \bar{B}^{0}$ mesons. After the lepton selection we reconstruct $D^{0} \rightarrow K^{-} \pi^{+}$signal around the negative lepton candidate. If a $D^{0}$ candidate is found, we then look for $D^{*+} \rightarrow D^{0} \pi_{s}^{+}$candidates by attaching another track as $\pi_{s}^{+}$to the $D^{0}$ candidate. Then the $D^{*+}$ candidates are removed from the original $D^{0}$ sample. Eventually two mutually exclusive samples are created in each lepton dataset, the $\ell^{-} D^{0}\left(D^{*+}\right.$ excluded $)$ sample and the $\ell^{-} D^{*+}$ sample.

\subsection{1 $D^{0}$ reconstruction}

For the $D^{0} \rightarrow K^{-} \pi^{+}$reconstruction, we examine all the oppositely charged track pairs as $K^{-}$and $\pi^{+}$. To reduce combinatorial background, and achieve good data quality for lifetime measurements, we apply following cuts for the $D^{0}$ selection.

- $K^{-}, \pi^{+}$track quality cuts

- Number of hits in COT axial (stereo) layers $\geq 20$ (16)

- Number of hits in SVX II $r$ - $\phi$ layers $\geq 3$

$-\left|\Delta z_{0}(\ell-K)\right|,\left|\Delta z_{0}(\ell-\pi)\right|<1.25 \mathrm{~cm}$

- Kinematic cuts

$$
\begin{aligned}
& -p_{T}(K)>1.5 \mathrm{GeV} / c, p_{T}(\pi)>0.5 \mathrm{GeV} / c \\
& -\Delta R(\ell-K)<0.6, \Delta R(\ell-\pi)<0.7 \\
& -M\left(\ell^{-} D^{0}\right)<5.3 \mathrm{GeV} / c^{2}
\end{aligned}
$$

where $M\left(\ell^{-} D^{0}\right)$ is invariant mass of the lepton- $D^{0}$ system, $\Delta R$ is defined as $\Delta R=\sqrt{\Delta \eta^{2}+\Delta \phi^{2}}$, where $\phi$ is given in unit of radian.

- Cuts for decay length and its uncertainties

$$
\begin{aligned}
& -L_{x y}\left(D^{0}-P . V .\right)>0 \\
& -\left|c t\left(D^{0}\right)\right|<0.1 \mathrm{~cm} \\
& --0.15<c t^{*}(B)<0.3 \mathrm{~cm} \\
& -\sigma_{c t^{*}}(B), \sigma_{c t}\left(D^{0}\right)<0.02 \mathrm{~cm}
\end{aligned}
$$


where $L_{x y}\left(D^{0}-P . V\right.$. $)$ is a decay length in $x y$ plane between $D^{0}$ decay point and Primary Vertex (P.V.) in the semileptonic $B$ decay chain, $c t^{(*)}$ and $\sigma_{c t^{(*)}}$ are (pseudo-)proper decay length of each meson and its uncertainty. Decay length reconstruction is described in detail in Section 3.6. An event display for a typical $\ell^{-} D^{0}$ candidate is shown in Figure 3.2.

In the semileptonic $B^{-}$and $\bar{B}^{0}$ decays, a negatively charged lepton is generated in associating with the $D^{0}$ meson, not with $\bar{D}^{0}$ meson. Since the $D^{0}$ meson decays exclusively into $K^{-} \pi^{+}(\mathrm{Br}=3.80 \pm 0.09 \%)$ but practically not to $K^{+} \pi^{-}(\mathrm{Br}=0.014 \pm 0.001 \%)$, it provides a useful charge correlation between the lepton and kaon charges. In the $D^{0}$ selection cuts, we require the right charge correlation. Here we refer the right sign charge correlation as RS, and wrong sign correlation as WS. From the $\ell^{-} D^{0}$ (all) sample, events which have mass difference $\Delta M$ in a $D^{*+}$ veto region $0.142-0.148 \mathrm{GeV} / c^{2}$ are rejected to form the $\ell^{-} D^{0}\left(D^{*+}\right.$ excluded) sample ( $\Delta M$ calculation is described in Section 3.4.2). Plots in Figures 3.3, 3.4 show $M\left(K^{-} \pi^{+}\right)$for the $\ell^{-} D^{0}\left(D^{*+}\right.$ excluded $)$ samples for muons, electrons, and muon and electron combined datasets. For the $\ell^{-} D^{0}\left(D^{*+}\right.$ excluded) sample, we define $M\left(K^{-} \pi^{+}\right)$signal region as $1.845-$ $1.885 \mathrm{GeV} / c^{2}$. We also define sideband regions as $1.74-1.79 \mathrm{GeV} / c^{2}$ (LSB) and $1.94-1.99 \mathrm{GeV} / c^{2}$ (USB). Events in the signal region are used as the $B$ decay signal sample, and sideband events are used as background control sample. To estimate the number of combinatorial background events under the $D^{0}$ mass peak, we fit a Gaussian plus second order polynomial function to the $M\left(K^{+} \pi^{-}\right)$distribution. From the number of events in signal region and expected number of background events obtained from the fit, we calculate signal fraction in the signal region $\left(f_{\text {sig }}\right)$ for $\ell^{-} D^{0}\left(D^{*+}\right.$ excluded $)$ sample, as summarized in Table 3.1.

\subsection{2 $D^{*+}$ reconstruction}

Now we attach a positively charged track as $\pi_{s}^{+}$to each $D^{0}$ (all) candidate that is in the mass window $1.835<M\left(K^{-} \pi^{+}\right)<1.895 \mathrm{GeV} / c^{2}$, and take a difference in the invariant masses $\Delta M=M\left(K^{-} \pi^{+} \pi_{s}^{+}\right)-M\left(K^{-} \pi^{+}\right)$. For the $\pi_{s}^{+}$candidate tracks we require the number of SVX II $r-\phi$ hits is greater than 3. Figures 3.3, 3.4 show the $\Delta M$ plots for the $\ell^{-} D^{*+}$ sample. We define signal and sideband regions for the $\ell^{-} D^{*+}$ samples as follows.

$\Delta M$ signal region : $0.144-0.147 \mathrm{GeV} / c^{2}$

$\Delta M$ sideband region : $0.15-0.2 \mathrm{GeV} / c^{2}$

We estimate the number of combinatorial background events in the $\Delta M$ signal region using $D^{0} \pi_{s}^{-}$events where the $\pi_{s}$ track has wrong charge. First we define 
a WS scale $S_{\text {WS }}$ using the $\Delta M$ sideband events, and estimate expected number of background events in the signal region.

$$
\begin{gathered}
S_{\mathrm{WS}}=\frac{N_{\mathrm{SB}}(\mathrm{RS})}{N_{\mathrm{SB}}(\mathrm{WS})} \\
N_{\mathrm{BG}}=N_{\mathrm{sig}}(\mathrm{WS}) \cdot S_{\mathrm{WS}}
\end{gathered}
$$

Note that with the $D^{*+}$ sample notations RS, WS are for $\pi_{s}^{ \pm} D^{0}$ charge correlation, not for the $\ell^{-} D^{0}$ system. From the number of events in the signal region and the estimated number of background events, the $D^{*+}$ fraction in the $\Delta M$ signal region $\left(f_{\text {sig }}\right)$ is calculated. Definitions of all the signal and sideband regions, and event yields are summarized in Tables 3.1 and 3.2.

\begin{tabular}{lclrl}
\hline \hline Sample & $M\left(K^{-} \pi^{+}\right)$range $\left(\mathrm{GeV} / c^{2}\right)$ & $\Delta M$ range $\left(\mathrm{GeV} / c^{2}\right)$ & Events & Signal fraction \\
\hline$\mu^{-} D^{0}$ & $1.845-1.885$ & $0.142-0.148($ veto $)$ & 21971 & $0.425 \pm 0.004$ \\
$\mu^{-} D^{*+}$ & $1.835-1.895$ & $0.144-0.147$ & 2947 & $0.907 \pm 0.007$ \\
$e^{-} D^{0}$ & $1.845-1.885$ & $0.142-0.148$ (veto) & 8539 & $0.471 \pm 0.007$ \\
$e^{-} D^{*+}$ & $1.835-1.895$ & $0.144-0.147$ & 1219 & $0.908 \pm 0.010$ \\
\hline \hline
\end{tabular}

Table 3.1: Summary of signal region definitions and yields for the $\ell^{-} D^{0}\left(D^{*+}\right.$ excluded) and the $\ell^{-} D^{*+}$ samples.

\begin{tabular}{lllr}
\hline \hline Sample & $M\left(K^{-} \pi^{+}\right)$range $\left(\mathrm{GeV} / c^{2}\right)$ & $\Delta M$ range $\left(\mathrm{GeV} / c^{2}\right)$ & Events \\
\hline$\mu^{-} D^{0}$ & $1.74-1.79,1.94-1.99$ & $0.142-0.148($ veto) & 32763 \\
$\mu^{-} D^{*+}$ & $1.835-1.895$ & $0.15-0.2$ & 7658 \\
$e^{-} D^{0}$ & $1.74-1.79,1.94-1.99$ & $0.142-0.148($ veto) & 11868 \\
$e^{-} D^{*+}$ & $1.835-1.895$ & $0.15-0.2$ & 2922 \\
\hline \hline
\end{tabular}

Table 3.2: Summary of sideband region definitions and yields for the $\ell^{-} D^{0}$ ( $D^{*+}$ excluded) and the $\ell^{-} D^{*+}$ samples. 


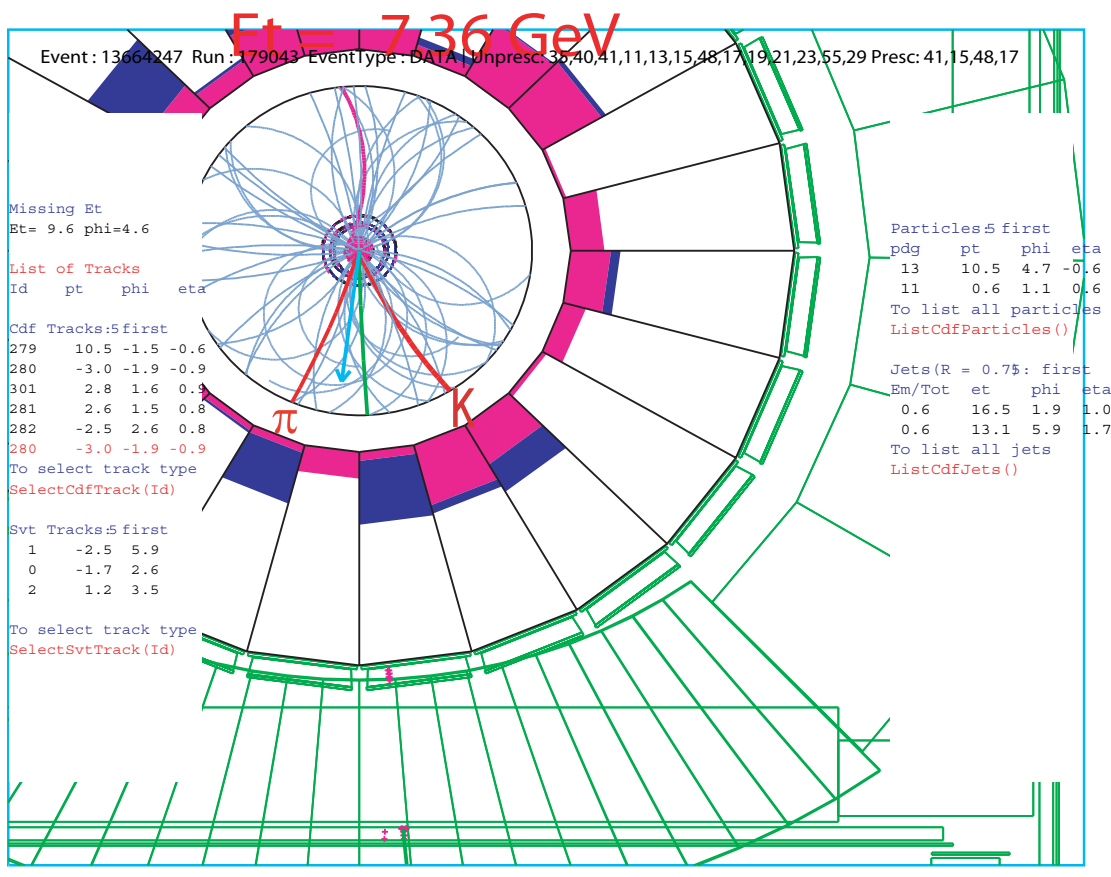

$\mu$

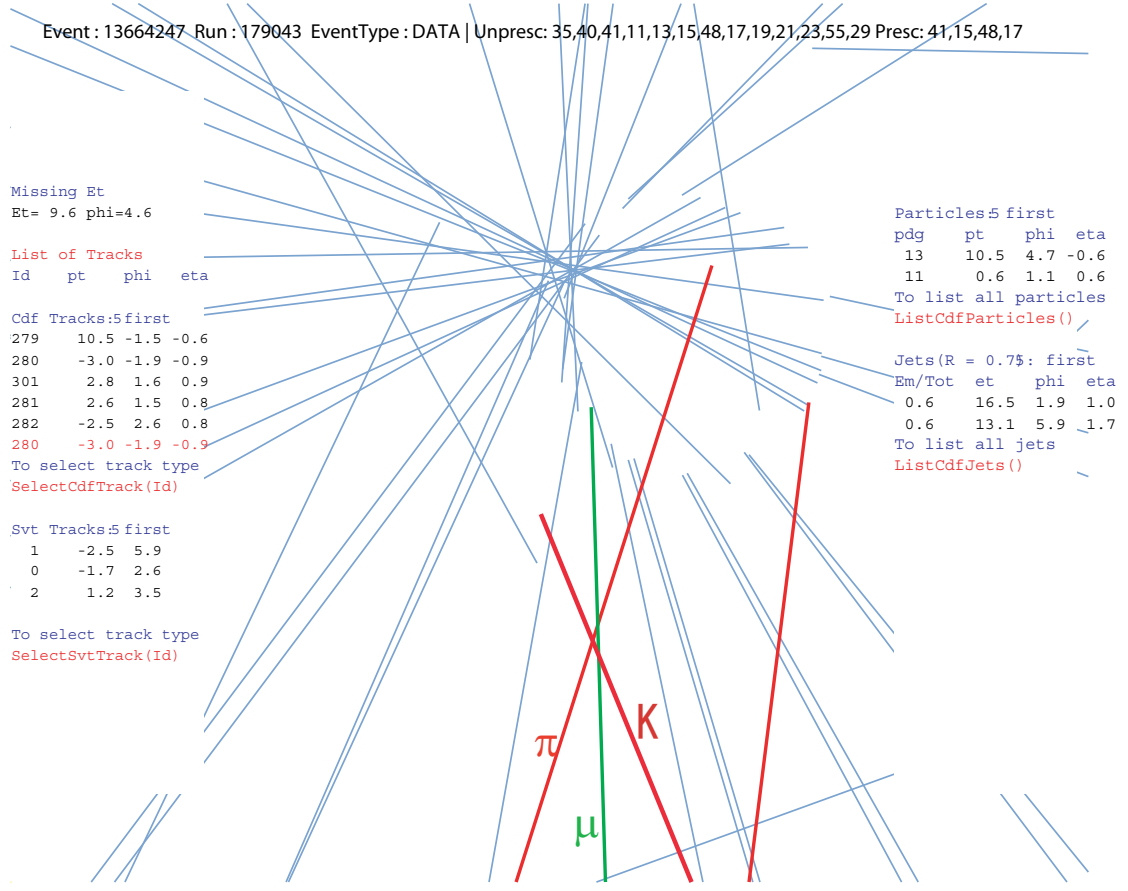

Figure 3.2: An event display of a typical $\ell^{-} D^{0}$ candidate. Lower figure is a close view of the upper figure around the collision point. 

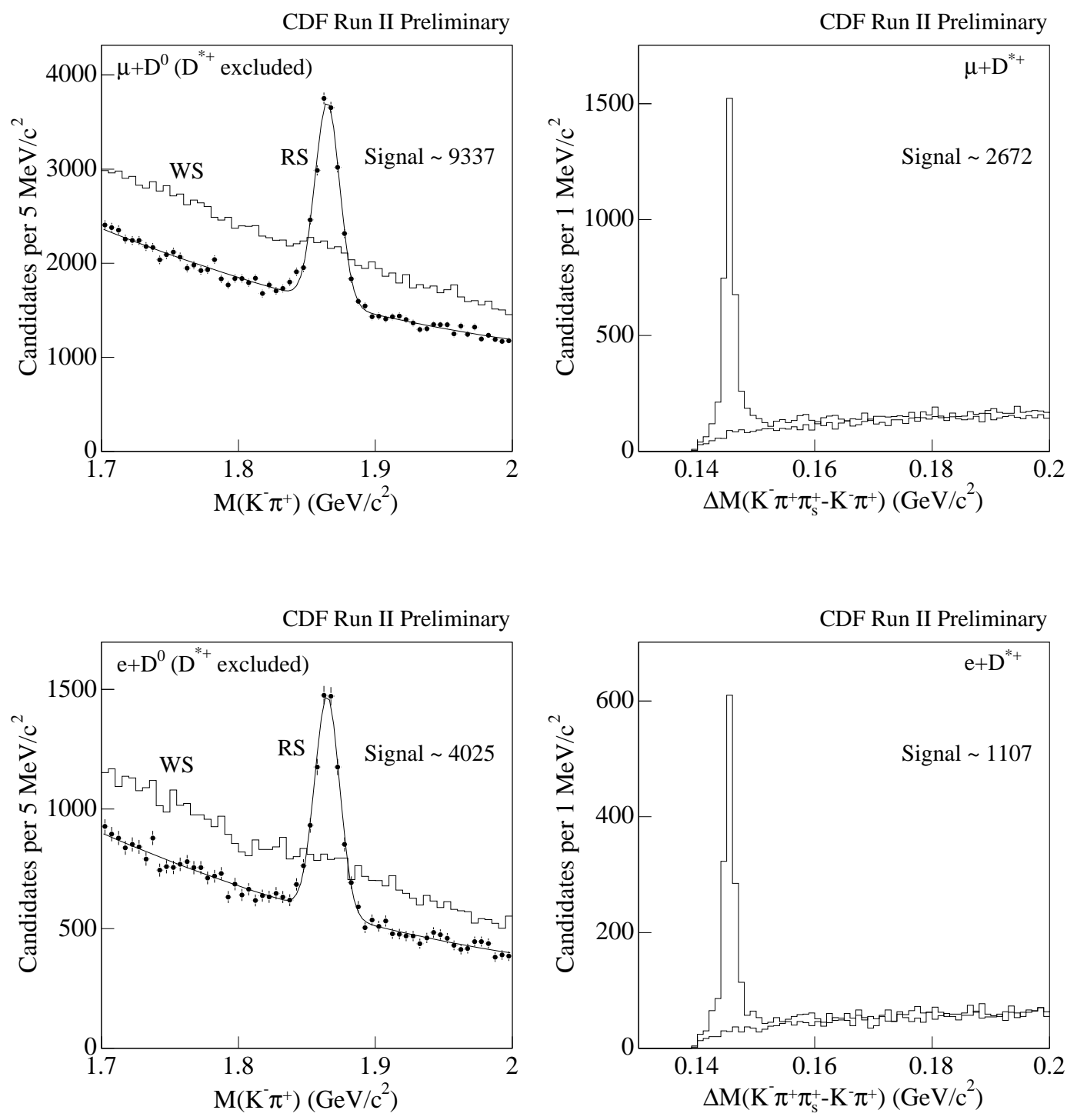

Figure 3.3: $M\left(K^{-} \pi^{+}\right)$plots for the $\ell^{-} D^{0}\left(D^{*+}\right.$ excluded) sample (left) and $\Delta M\left(K^{-} \pi^{+} \pi_{s}^{+}-K^{-} \pi^{+}\right)$plots for the $\ell^{-} D^{*+}$ sample. In the $M\left(K^{-} \pi^{+}\right)$plots, box histograms show events which have wrong charge correlation between lepton and kaon. In the $\Delta M$ plots, histograms for events where $\pi_{s}$ track has wrong charge are also shown. Upper and lower plots are for the muon and electron datasets, respectively. 

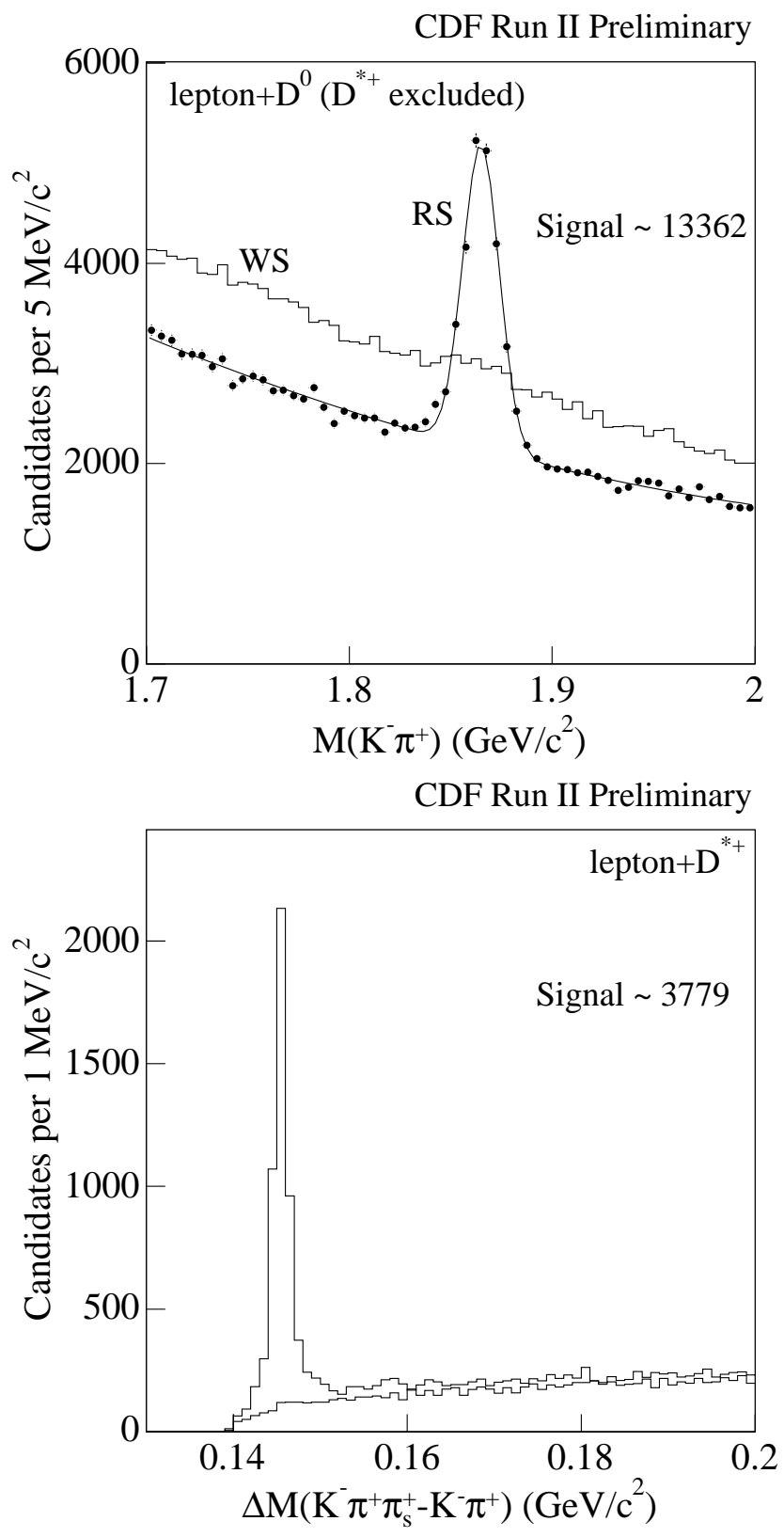

Figure 3.4: $M\left(K^{-} \pi^{+}\right)$and $\Delta M\left(K^{-} \pi^{+} \pi_{s}^{+}-K^{-} \pi^{+}\right)$plots for the $\ell^{-} D^{0}\left(D^{*+}\right.$ excluded) and the $\ell^{-} D^{*+}$ samples (muon and electron combined dataset). 


\subsection{Reconstruction of the $D_{s}^{+}$mesons}

Then we turn to reconstruct the $\ell^{-} D_{s}^{+}$signal to identify the $\bar{B}_{s}^{0}$ semileptonic decays. We look for the $D_{s}^{+} \rightarrow \phi(1020) \pi^{+}$signal $(\mathrm{Br}=3.6 \pm 0.9 \%)$ around the negative lepton candidate. Most part of the selection cuts and signal region definition are set to achieve the best statistical power for the lifetime measurement.

\subsection{1 $\phi(1020) \rightarrow K^{+} K^{-}$reconstruction}

First we take any oppositely charged track pairs as the $K^{+} K^{-}$candidates, and reconstruct their invariant mass to obtain the $\phi \rightarrow K^{+} K^{-}$decay signal $(\mathrm{Br}=49.1 \pm 0.6 \%)$. Following are the selection criteria for the charged kaon candidates from the $\phi$ mesons.

- $p_{T}(K)>1.2 \mathrm{GeV} / c$

- $\Delta R(\ell-K)<0.8$

- $\left|\Delta z_{0}(\ell-K)\right|<1 \mathrm{~cm}$

Obtained $K^{+} K^{-}$mass spectra are displayed in Figure 3.7. We define the $M\left(K^{+} K^{-}\right)$signal region as $1019.456 \pm 7 \mathrm{MeV} / c^{2}$, where the $1019.456 \mathrm{MeV} / c^{2}$ is the $\phi$ meson mass from the Particle Data Book [1].

\subsection{2 $D_{s}^{+} \rightarrow \phi \pi^{+}$reconstruction}

To the $\phi$ candidates, we attach any positively charged tracks as $\pi^{+}$candidates, and calculate the $K^{+} K^{-} \pi^{+}$invariant mass to form the $D_{s}^{+} \rightarrow \phi \pi^{+}$candidates. In addition to the $\phi$ selection cuts, following cuts are applied to the $D_{s}^{+}$ candidates.

- $p_{T}\left(\pi^{+}\right)>0.9 \mathrm{GeV} / c$

- $\Delta R\left(\ell-\pi^{+}\right)<0.8$

- $\left|\Delta z_{0}\left(\ell-\pi^{+}\right)\right|<1 \mathrm{~cm}$

- $3<M\left(\ell^{-} K^{+} K^{-} \pi^{+}\right)<5.4 \mathrm{GeV} / c^{2}$

- $|\cos \psi|>0.4$ 
where $\psi$ is the helicity angle between $K^{+}$and $\pi^{+}$in the $\phi$ meson rest frame. Figure 3.5 schematically shows definition of the helicity angle $\psi$. In the decay $D_{s}^{+} \rightarrow \phi \pi^{+}$, both $D_{s}^{+}$and $\pi^{+}$have $J=0$ while the $\phi$ meson have $J=1$. So, on the $\phi$ meson rest frame, taking the $z$-axis along the $\pi^{+}$flight direction, initial state $\left(D_{s}^{+}\right)$has $J=0, J_{z}=0$. Considering angular momentum conservation, the $\phi \pi^{+}$system must have $J=0, J_{z}=L_{z}+S_{z}=0$, where $L_{z}$ and $S_{z}$ are $z$ component of the orbital angular momentum and spin of the $\phi \pi^{+}$system. The $\phi \pi^{+}$system has $p_{x}, p_{y}=0$, thus $L_{z}=0, S_{z}=0$. So the $\phi$ meson is a pure $J=1, J_{z}=0$ state. In the decay $\phi \rightarrow K^{+} K^{-}$, the kaons have $J=0$, so the kaons system has $L=1, L_{z}=0$. Thus the angular distribution is proportional to $Y_{1}^{0} \propto \cos ^{2} \psi$. The $\cos \psi$ and $M\left(\ell^{-} K^{+} K^{-} \pi^{+}\right)$distributions for muon and electron combined sample are shown in Figure 3.6.

After applying these cuts, we cut on the decay length information and vertex fit probability.

- $L_{x y}\left(D_{s}^{+}-P . V.\right)>0$

- $\left|\operatorname{ct}\left(D_{s}^{+}\right)\right|<0.1 \mathrm{~cm}$

- $\log ($ fit prob. $)>-10$

- $\sigma_{c t^{*}}\left(\bar{B}_{s}^{0}\right), \sigma_{c t}\left(D_{s}^{+}\right)<0.02 \mathrm{~cm}$

- $-0.15<c t^{*}\left(\bar{B}_{s}^{0}\right)<0.3 \mathrm{~cm}$

where $L_{x y}\left(D_{s}^{+}-P . V\right.$. $)$ is a decay length between $D_{s}^{+}$decay point and Primary Vertex $\left(P . V\right.$.) in the semileptonic $\bar{B}_{s}^{0}$ decay chain. The fit probability is calculated at the vertex fit (see Section 3.6). Like the $\ell^{-} D^{0}$ case, in the semileptonic $\bar{B}_{s}^{0}$ decay a negatively charged lepton is generated in associating with the $D_{s}^{+}$ meson, not with $D_{s}^{-}$meson. Since the $D_{s}^{+}$meson decays into $\phi \pi^{+}$but not into $\phi \pi^{-}$, a charge correlation between the lepton and pion is available. In addition to all the selection cuts, we require the right charge correlation.

Plots in Figure 3.7 show the $M\left(K^{+} K^{-} \pi^{+}\right)$distributions both for muon and electron datasets. There are $D_{s}^{+}$peaks at $M=1967.5 \mathrm{MeV} / c^{2}$ in Right Sign (RS) events, which are not seen in the Wrong Sign (WS) events. There are also small peaks observed at $M=1869 \mathrm{MeV} / c^{2}$, which come from cabbibosuppressed $D^{+} \rightarrow \phi \pi^{+}$decay. We define the $M\left(K^{+} K^{-} \pi^{+}\right)$signal region to be $1967.5 \pm 20 \mathrm{MeV} / c^{2}$, where the $1967.5 \mathrm{MeV} / c^{2}$ is the $D_{s}^{+}$meson mass obtained from the fit to the mass plots. The sideband regions are also defined as $1900-1930 \mathrm{MeV} / c^{2}$ (LSB), and $2015-2035 \mathrm{MeV} / c^{2}$ (USB). To estimate the number of combinatorial background events under the $D_{s}^{+}$mass peak, we fit the mass plots with a function which is a sum of two Gaussian peaks (for $D_{s}^{+}$ and $D^{+}$peaks) and a second order polynomial. We fix the differences of two 
Gaussian peaks to be the $M\left(D_{s}^{+}\right)-M\left(D^{+}\right)$World Average $\left(98.87 \mathrm{MeV} / c^{2}\right)$, and constrain the relation of both width to be $\sigma_{D^{+}} / \sigma_{D_{s}^{+}}=M\left(D^{+}\right) / M\left(D_{s}^{+}\right)=$ $1-98.87 / M\left(D_{s}^{+}\right)$. From the number of events in the signal region and expected number of background events obtained from the mass fit, we calculate $D_{s}^{+}$ signal fraction $\left(f_{\text {sig }} \equiv \frac{N_{D_{s}^{+}}}{N_{\text {event }}}\right)$ in the signal region. The event yields and signal fractions are summarized in Table 3.3. The $M\left(K^{+} K^{-}\right)$and $M\left(K^{+} K^{-} \pi^{+}\right)$ plots for the electron and muon combined sample is also shown in Figure 3.8.

\begin{tabular}{lcll}
\hline \hline Sample & $M\left(K^{+} K^{-} \pi^{+}\right)$range $\left(\mathrm{MeV} / c^{2}\right)$ & Events & $D_{s}^{+}$signal fraction \\
\hline$\mu^{-} D_{s}^{+}$signal & $1947.5-1987.5$ & 1591 & $0.503 \pm 0.014$ \\
$\mu^{-} D_{s}^{+}$sideband & $1900-1930,2005-2035$ & 1181 & \\
$e^{-} D_{s}^{+}$signal & $1947.5-1987.5$ & 706 & $0.503 \pm 0.021$ \\
$e^{-} D_{s}^{+}$sideband & $1900-1930,2005-2035$ & 535 & \\
\hline \hline
\end{tabular}

Table 3.3: Summary of signal, sideband region definitions and event yields for the $\ell^{-} D_{s}^{+}$samples.

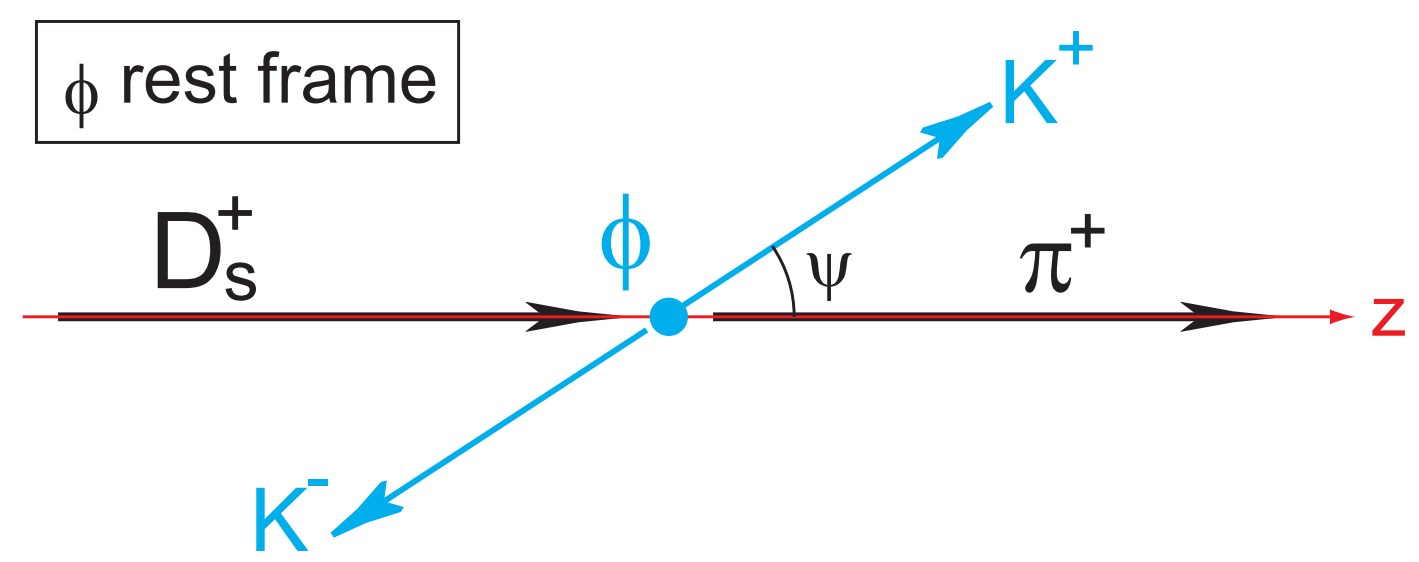

Figure 3.5: A schematic drawing which shows the definition of helicity angle $\psi$. 

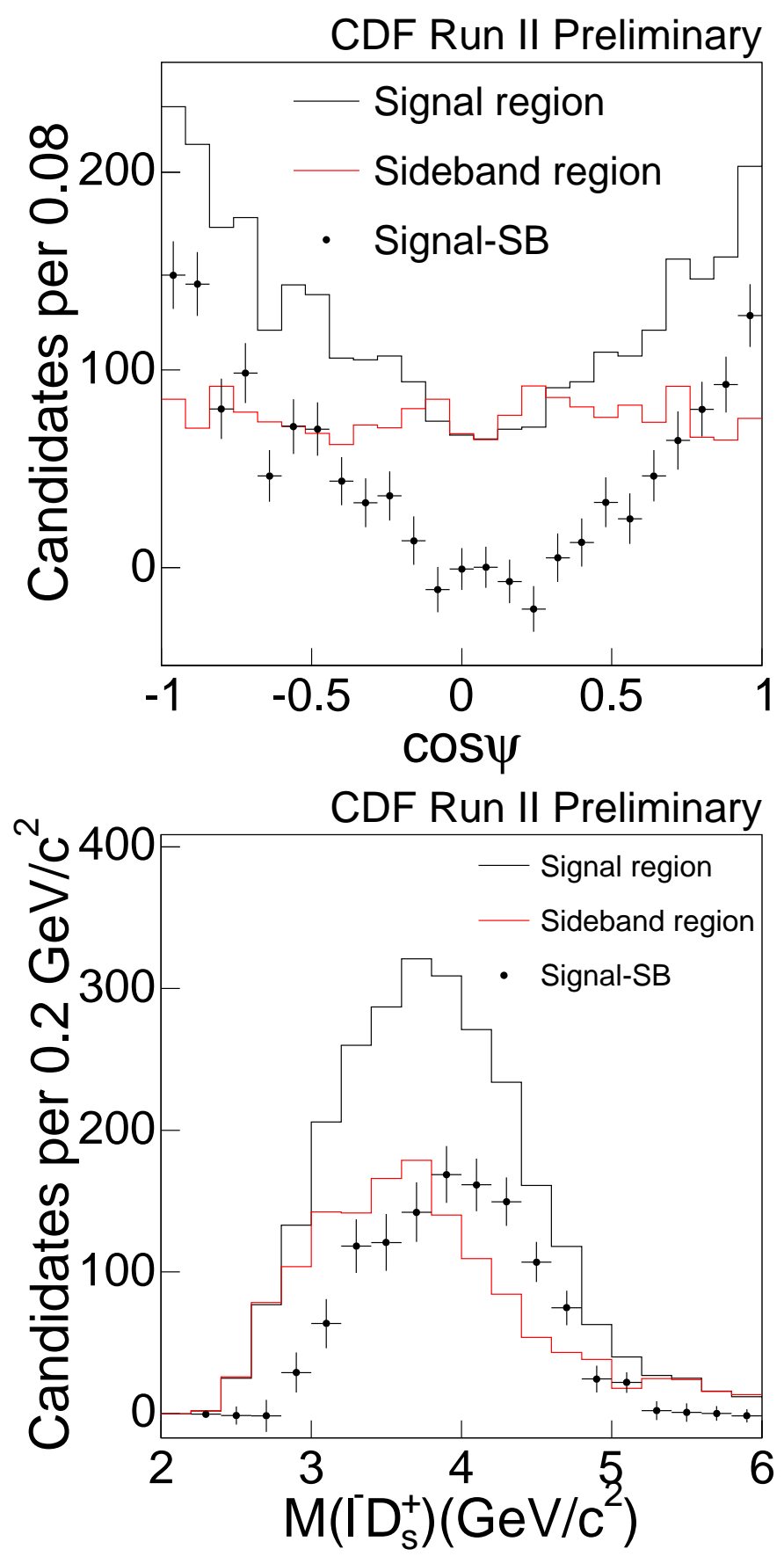

Figure 3.6: The $\cos \psi$ and $M\left(\ell D_{s}^{+}\right)$distributions for the $\ell^{-} D_{s}^{+}$(muon and electron combined) sample. Distributions for signal, sideband region events are shown as well as the difference between them. 

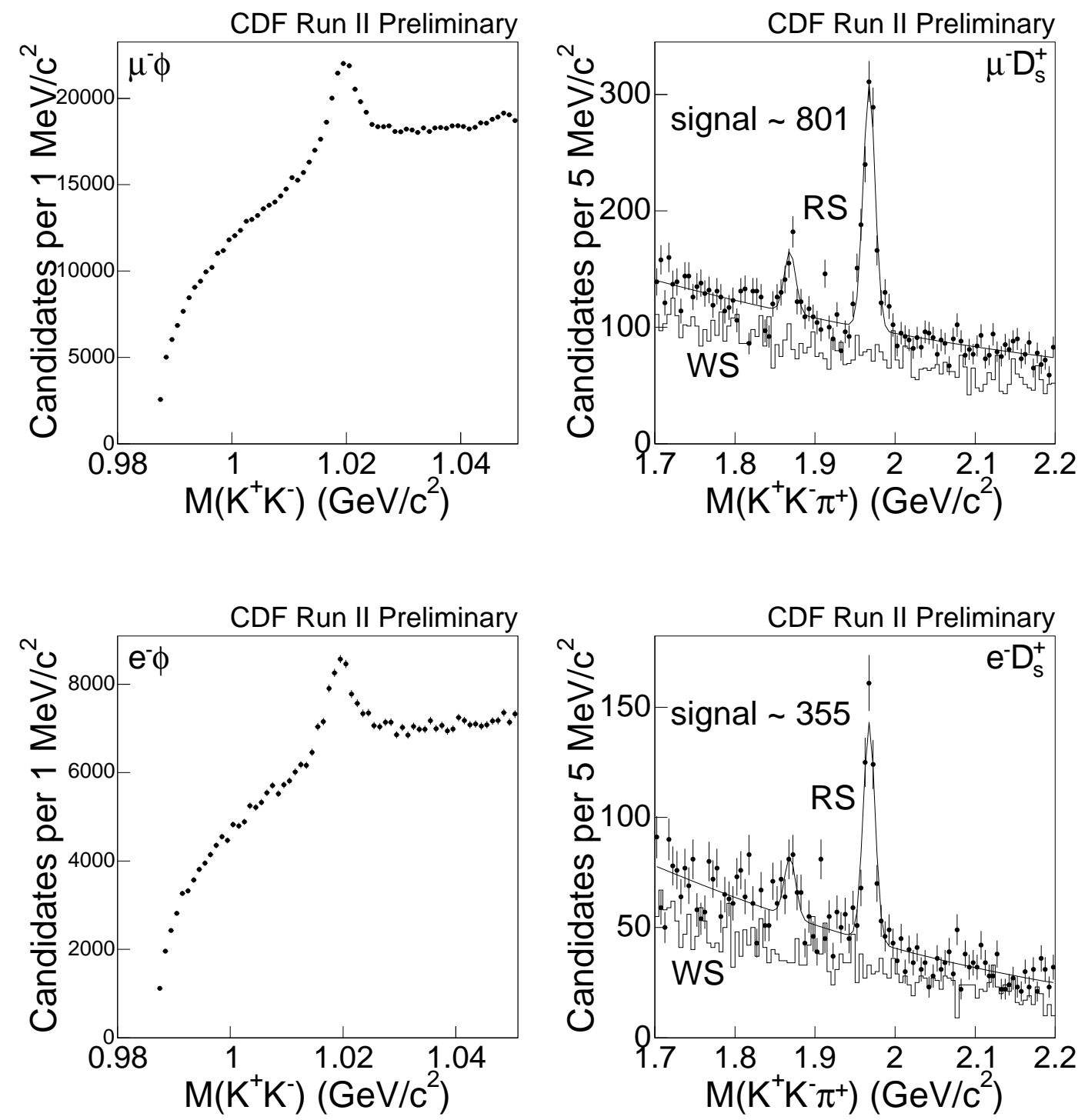

Figure 3.7: $M\left(K^{+} K^{-}\right)$and $M\left(K^{+} K^{-} \pi^{+}\right)$plots for the $\ell^{-} D_{s}^{+}$sample. In the $M\left(K^{+} K^{-} \pi^{+}\right)$plots, points show events which have right sign charge correlation between lepton and pion, while histograms show events with wrong charge correlation. Upper and lower plots are for the muon and electron datasets respectively. 

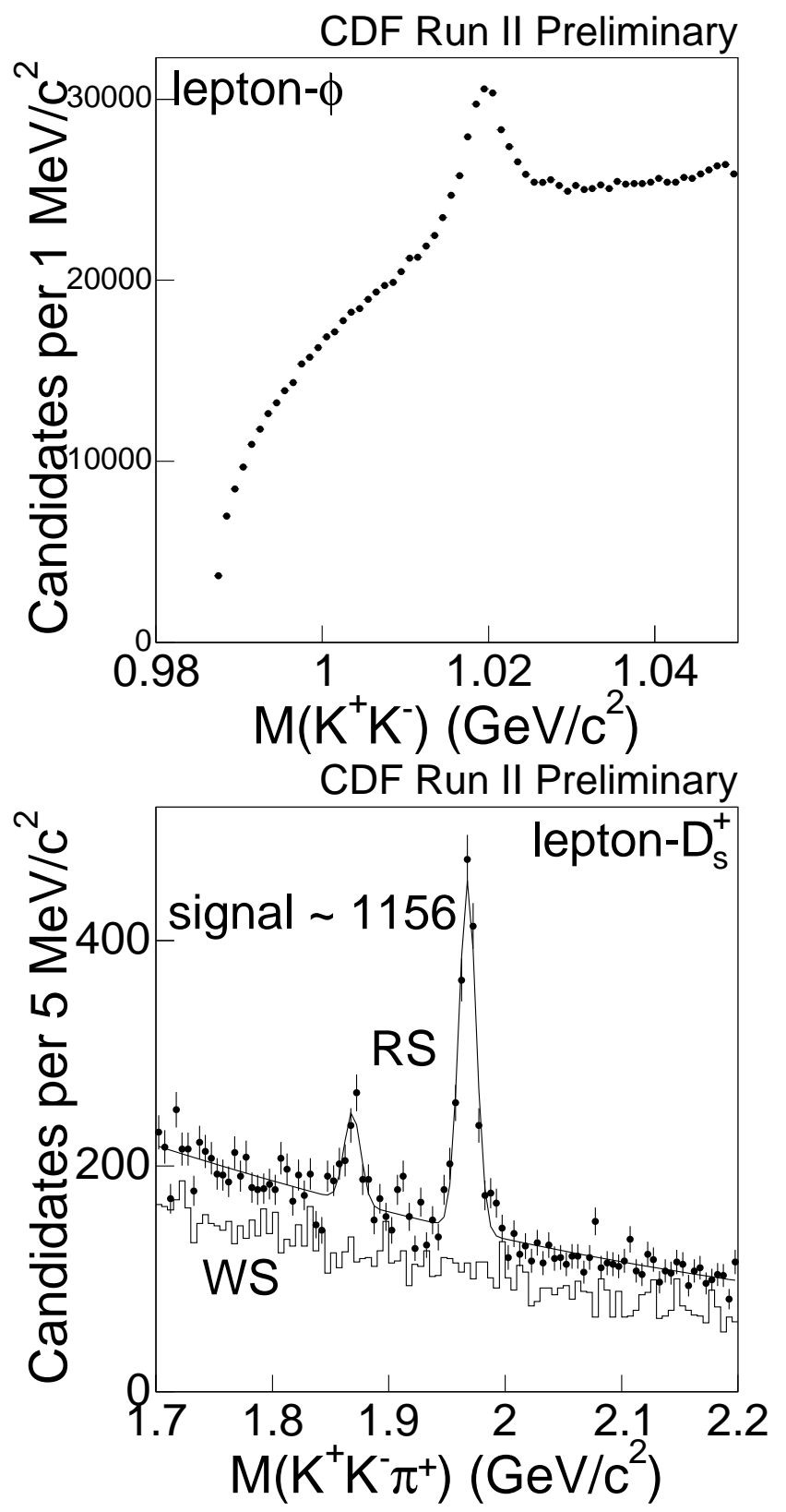

Figure 3.8: $M\left(K^{+} K^{-}\right)$and $M\left(K^{+} K^{-} \pi^{+}\right)$plots for muon and electron combined sample. 


\subsection{Reconstruction of the $B$ meson decays}

We now move to the $B$ decay time measurement. $B$ semileptonic decay point is reconstructed from the tracks appeared in the final state. A schematic view of the $B$ decay vertex reconstruction is shown in Figure 3.9 for $B^{-}$, and in Figure 3.10 for $\bar{B}_{s}^{0}$ case. Throughout in this section, combination of $B$ and $D$ denotes $B^{-} / \bar{B}^{0}$ and $D^{0}$ for $\ell^{-} D^{*+}$ or $D^{0}$ sample, or $\bar{B}_{s}^{0}$ and $D_{s}^{+}$for $\ell^{-} D_{s}^{+}$ sample.

To calculate the $B$ and $D$ decay points precisely, we perform so-called "vertex fit" [56]. Here we describe briefly about the vertex fit. Firstly from the five track parameters and another parameter $s$, which is a length along the track, we can write the $\phi$ value on the position at $s$ along the track as follows.

$$
\phi(s)=\frac{s}{r}+\phi_{0}=2 c s+\phi_{0}
$$

Then, the $x, y$, and $z$ coordinates of the track are obtained from any $s$ through the following equations.

$$
\begin{aligned}
& x=r \sin \phi-\left(r+d_{0}\right) \sin \phi_{0} \\
& y=-r \cos \phi+\left(r+d_{0}\right) \cos \phi_{0} \\
& z=z_{0}+s \lambda
\end{aligned}
$$

These equations can be solved for $s, d_{0}$, and $z_{0}$ as,

$$
\begin{aligned}
s & =\frac{1}{2 c} \sin ^{-1}\left[2 c\left(x \cos \phi_{0}+y \sin \phi_{0}\right)\right] \\
d_{0} & =y \cos \phi_{0}-x \sin \phi_{0}-\frac{1}{c} \sin ^{2} c s \\
z_{0} & =z-\lambda s
\end{aligned}
$$

The goal of the vertex fit is to find the vertex point $\left(x_{s}, y_{s}, z_{s}\right)$ together with the fitted track parameters, so that the vertex lies on the respective tracks. The procedure applied in the vertex fit to find such a vertex point is a minimization of the following $\chi^{2}$,

$$
\chi^{2}=\sum_{i=1}^{N} \xi_{i}^{T} G_{i}^{-1} \xi_{i},
$$

where $G$ is the error matrix of the measured parameters, and $\xi$ is a vector containing the differences between the track parameters as constrained by above equations and the actually measured parameters. $N$ is the number of fitted tracks.

The $B$ decay length $L_{B}$ and its uncertainty $\sigma_{L_{B}}$ measured in $x y$ plane are calculated using the vertex fit. The $B$ decay length $L_{B}$ is defined as the 
projection of $\vec{V}_{B}$ into $\vec{P}_{T}\left(\ell^{-} D\right)$ direction, as

$$
L_{B}=\vec{V}_{B} \cdot \frac{\vec{P}_{T}\left(\ell^{-} D\right)}{\left|\vec{P}_{T}\left(\ell^{-} D\right)\right|},
$$

where $\vec{V}_{B}$ is the $B$ decay vertex. The Primary Vertex position in $x y$ plane is taken from the colliding beam position. From $L_{B}$ and transverse momentum of the $\ell^{-} D$ system, we calculate pseudo-proper decay length $c t^{*}$ for each $\ell^{-}$Dcandidate.

$$
c t^{*}(B)=L_{B} \frac{M_{B}}{p_{T}\left(\ell^{-} D\right)},
$$

where $M_{B}$ is nominal $B$ meson mass taken from the Particle Data Book [1].

In addition to them, following quantities are calculated to use for the event selection described in Section 3.4.1.

$$
\begin{gathered}
L_{x y}(D-P . V .)=\vec{V}_{D} \cdot \frac{\vec{P}_{T}(D)}{\left|\vec{P}_{T}(D)\right|}, \\
L_{D}=\left(\vec{V}_{D}-\vec{V}_{B}\right) \cdot \frac{\vec{P}_{T}(D)}{\left|\vec{P}_{T}(D)\right|}, \\
c t(D)=L_{D} \frac{M_{D}}{p_{T}(D)},
\end{gathered}
$$

where $\vec{V}_{D}$ is a vector from $D$ decay vertex to the P.V., and $M_{D}$ is nominal $D$ meson mass. 


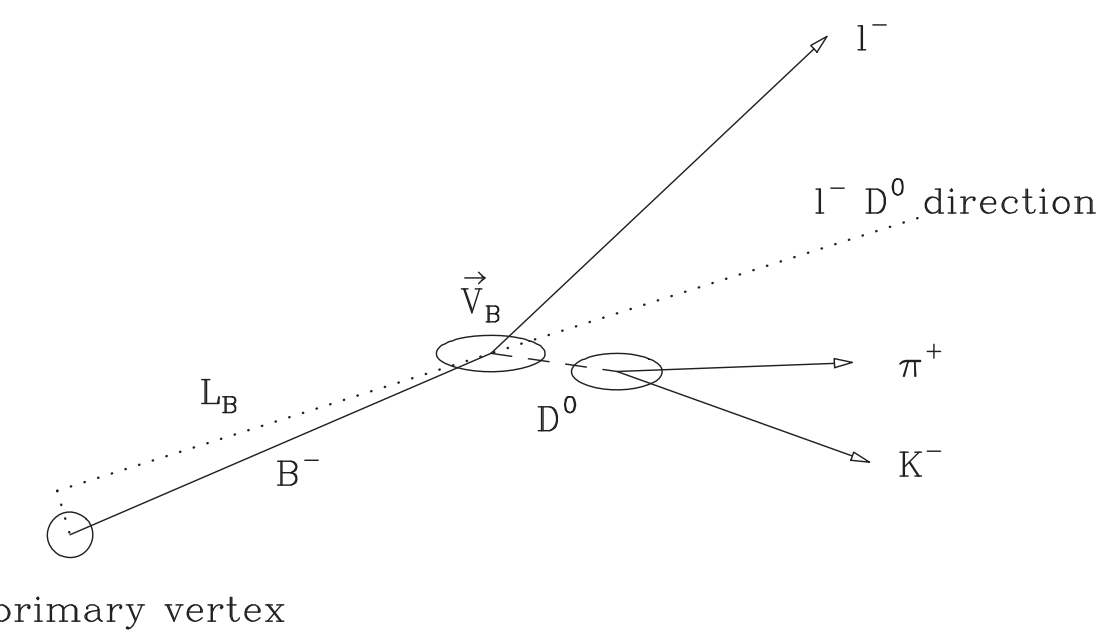

Figure 3.9: Schematic view of typical semileptonic decay $B^{-} \rightarrow \ell^{-} \bar{\nu} D^{0}, D^{0} \rightarrow$ $K^{-} \pi^{+}$in $x y$ plane.

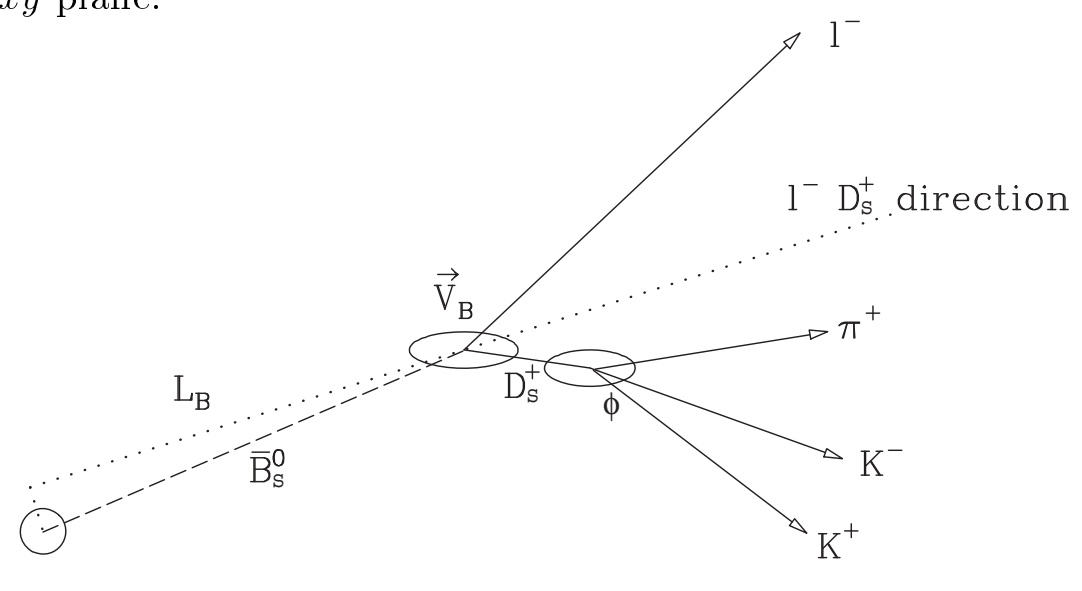

primary vertex

Figure 3.10: Schematic view of typical semileptonic decay $\bar{B}_{s}^{0} \rightarrow \ell^{-} \bar{\nu} D_{s}^{+}, D_{s}^{+} \rightarrow$ $\phi \pi^{+}, \phi \rightarrow K^{+} K^{-}$in $x y$ plane. 



\section{Chapter 4}

\section{Measurement of the $B^{-} / \bar{B}^{0}$ meson lifetimes}

In his chapter we describe the measurement of $B^{-} / \bar{B}^{0}$ meson lifetimes using the lepton $+D^{0}$ ( $D^{*+}$ excluded) and the $\ell^{-} D^{*+}$ samples. The strategy of the $B^{-} / \bar{B}^{0}$ analysis is commonly used for the $\bar{B}_{s}^{0}$ lifetime analysis which is described in the next chapter.

\section{1 $\quad B^{-} / \bar{B}^{0}$ Monte Carlo samples}

At first we discuss about the $B^{-} / \bar{B}^{0}$ signal Monte Carlo simulation samples, which are indispensable for the $B^{-} / \bar{B}^{0}$ meson lifetime measurement. In the simulation $B^{-} / \bar{B}^{0}$ mesons are generated with $p_{T}$ spectrum from theoretical calculation by NDE [57][63], and for the semileptonic decays ISGW model [58][62] is employed. We have generated $B^{-} / \bar{B}^{0}$ Monte Carlo samples under two different configurations. First one is parametric simulation which just keeps generator-level information. Second one is Monte Carlo sample with full detector simulation [60]. Two same numbers of the $B^{-}$and $\bar{B}^{0}$ mesons are generated, and these are forced to decay semileptonically $\left(\bar{B} \rightarrow \ell^{-} \bar{\nu} D X\right.$, where $D$ denotes neutral or charged, $J=0,1$ or 2 charm meson, $\ell^{-}$is $\mu^{-}$or $e^{-}$). Generated events which contain lepton and $D^{0}$ are picked up. The $D^{0}$ decays are forced into $K^{-} \pi^{+}$.

For the parametric simulation sample we apply some kinematic cuts as listed below on the parameters which are from the generator and do not include resolution effect.

- $|\eta(\ell)|<0.7,|\eta(K, \pi)|<1.1$

- $p_{T}(\ell)>8 \mathrm{GeV} / c$ 
- $p_{T}(K)>1.5 \mathrm{GeV} / c, p_{T}(\pi)>0.5 \mathrm{GeV} / c$

- $\Delta R(\ell-K)<0.6, \Delta R(\ell-\pi)<0.7$

- $\operatorname{ct}(B)<0.3 \mathrm{~cm}$

The parametric simulation sample is used for the muon $K$ factor calculation described in Section 4.2.

For the another sample, we apply full detector simulation for the generated events. After applying the detector simulation, the sample is processed by semileptonic $B^{-} / \bar{B}^{0}$ signal reconstruction module as done in the real data. All the cuts used for the real data are also applied. This full simulation sample is used for electron $K$ factor calculation (section 4.2). And also used for sample composition estimation (section 4.6) both for the electron and muon datasets.

\subsubsection{Tuning of $p_{T}(B)$ spectrum}

At the $B^{-} / \bar{B}^{0}$ meson generation the $p_{T}(b)$ spectrum based on NDE calculation (Nason, Dawson, Ellis [63]) is assumed. But it is known that the real $p_{T}(B)$ spectrum from $p \bar{p}$ collision has somewhat different shape from the NDE spectrum. We correct this difference using the $p_{T}(B)$ spectrum measured from the $J / \psi$ data in CDF Run II [61]. Figure 4.1 upper plot shows a comparison of the $p_{T}(B)$ spectra which are from the NDE calculation and from the measurement. The real $p_{T}(B)$ spectrum falls down steeper than the NDE spectrum. To achieve a good agreement between these spectra, we apply correction factor $p_{T}(B)^{\alpha}$ to the NDE spectrum. Using least $\chi^{2}$ method we look for the value $\alpha$ that gives a best match. Since most of $B^{-} / \bar{B}^{0}$ mesons which pass our selection cuts have $p_{T}(B)$ greater than $10 \mathrm{GeV} / c$, we use a region $10<p_{T}(B)<25 \mathrm{GeV} / c$ to fit the best value of $\alpha$. As a result of the fit, we find $\alpha=-0.7320 \pm 0.0001$. Figure 4.1 lower plot shows a comparison after applying the correction $p_{T}(B)^{-0.7320}$ to the NDE spectrum. Now the two spectra show a good agreement.

In the analysis all the events in the $B^{-} / \bar{B}^{0}$ Monte Carlo samples are weighted event by event with a factor of $p_{T}(B)^{-0.7320}$. 

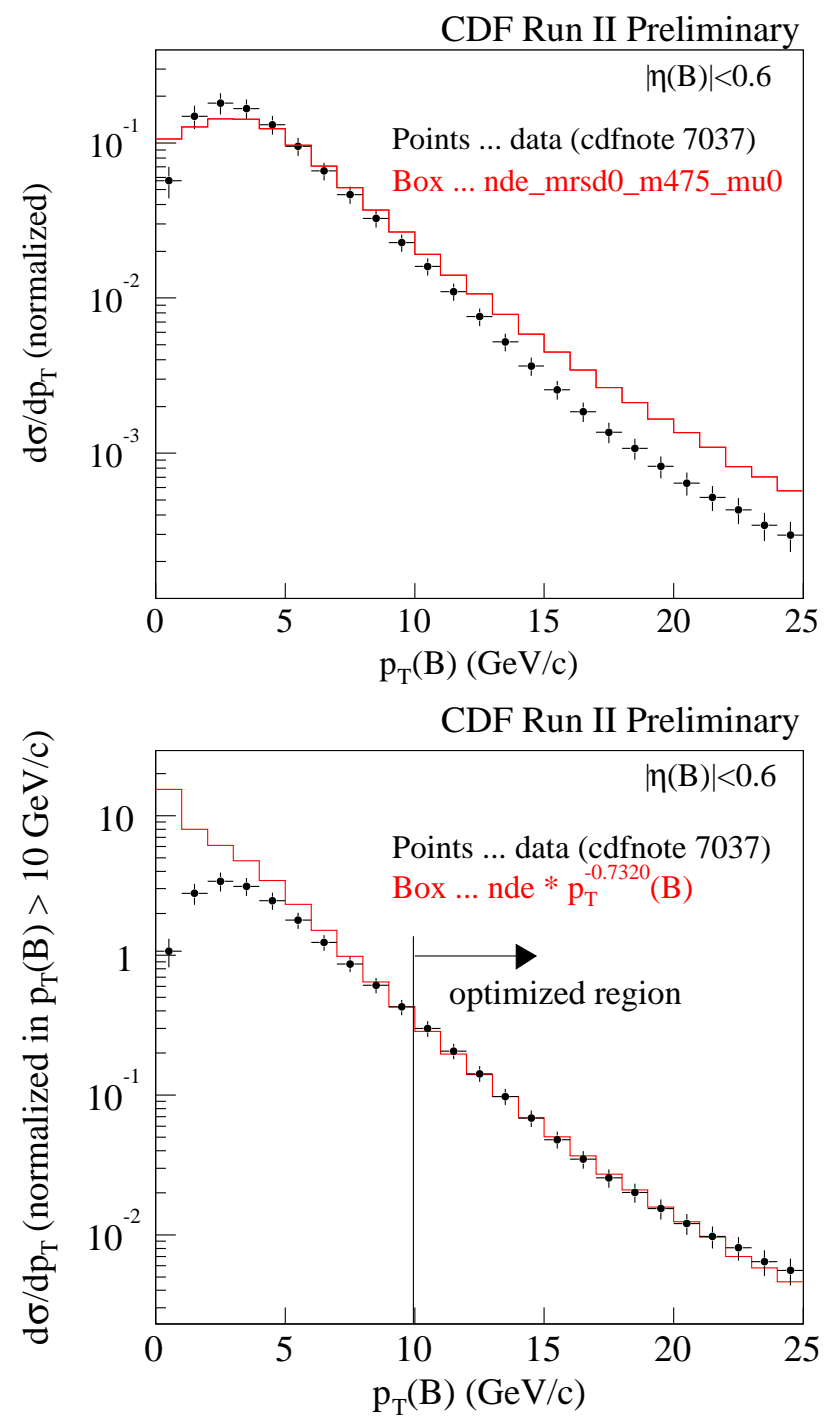

Figure 4.1: Comparison of the $B^{-} / \bar{B}^{0}$ meson transverse momentum spectra between the NDE calculation and the Run II measurement using $B \rightarrow J / \psi X$. Points show the measured spectrum, and red histogram is the NDE spectrum. Upper plot is a comparison before tuning the NDE spectrum, while lower plot is after tuning. 


\subsection{Missing momentum correction}

On the calculation of the $B$ proper decay length $\operatorname{ct}(B)$, decay length $L_{B}$ needs to be corrected by Lorentz Factors shown as follows.

$$
\operatorname{ct}(B)=\frac{L_{B}}{\beta \gamma}=L_{B} \frac{M_{B}}{p_{T}(B)},
$$

where $M_{B}$ is the nominal $B^{-} / \bar{B}^{0}$ meson mass taken from the World Average [1], and $\beta$ and $\gamma$ have their usual meanings. We can not fully reconstruct the transverse momentum of the $B^{-} / \bar{B}^{0}$ meson in the semileptonic $B^{-} / \bar{B}^{0}$ decays, but can only measure $p_{T}\left(\ell^{-} D^{0}\right)$. Consequently we define "pseudoproper" decay length $c t^{*}$ as follows.

$$
c t^{*}(B)=L_{B} \frac{M_{B}}{p_{T}\left(\ell^{-} D^{0}\right)}
$$

To convert $c t^{*}$ to $c t$, we define " $K$ factor" as the ratio of transverse momenta of the $\ell^{-} D^{0}$ system and $B^{-} / \bar{B}^{0}$ meson.

$$
c t^{*}(B)=c t \cdot \frac{p_{T}\left(\ell^{-} D^{0}\right)}{p_{T}(B)}=c t \cdot \frac{1}{K}, \quad \text { where } K \equiv \frac{p_{T}\left(\ell^{-} D^{0}\right)}{p_{T}(B)}
$$

The $K$ factor distributions are computed from the $B^{-} / \bar{B}^{0}$ Monte Carlo sample described in the previous section. For the muon dataset, we use the parametric simulation sample because it has much more statistics than the full simulation sample. For the electron dataset, we use the full simulation sample because some of the electron ID cuts possibly give some effects to the $K$ distribution.

In each for the electron and muon cases, the $B^{-} / \bar{B}^{0}$ Monte Carlo sample is divided into four subsamples, according to the $B^{-} / \bar{B}^{0}$ meson species and whether the daughter charm meson is $D^{0}\left(D^{*+}\right.$ excluded) or $D^{*+}$. From these subsamples, we calculate the $K$ factor distributions. The $K$ factor distributions are displayed in Figure 4.2 for the muon, and in Figure 4.3 for the electron dataset.

As a check, we also made the muon $K$ factor distributions from a full simulation sample. But the $K$ distributions both from the parametric and the full detector simulation samples are almost same in shapes and have consistent mean values. Therefore using the parametric simulation samples is verified for the muon $K$ factor calculation. It is not the for the electron. 

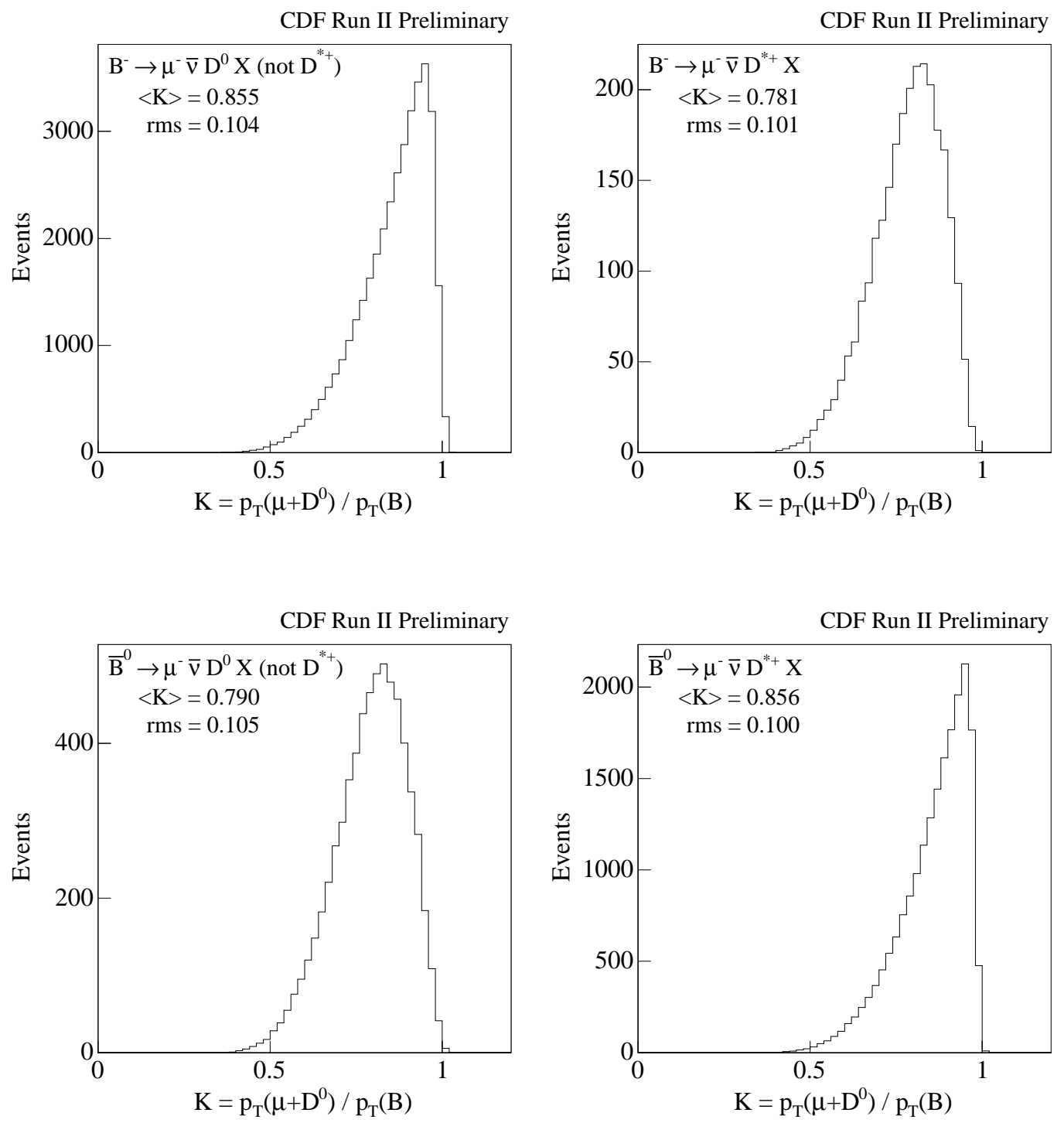

Figure 4.2: $K$ factor distributions for the muon dataset. 

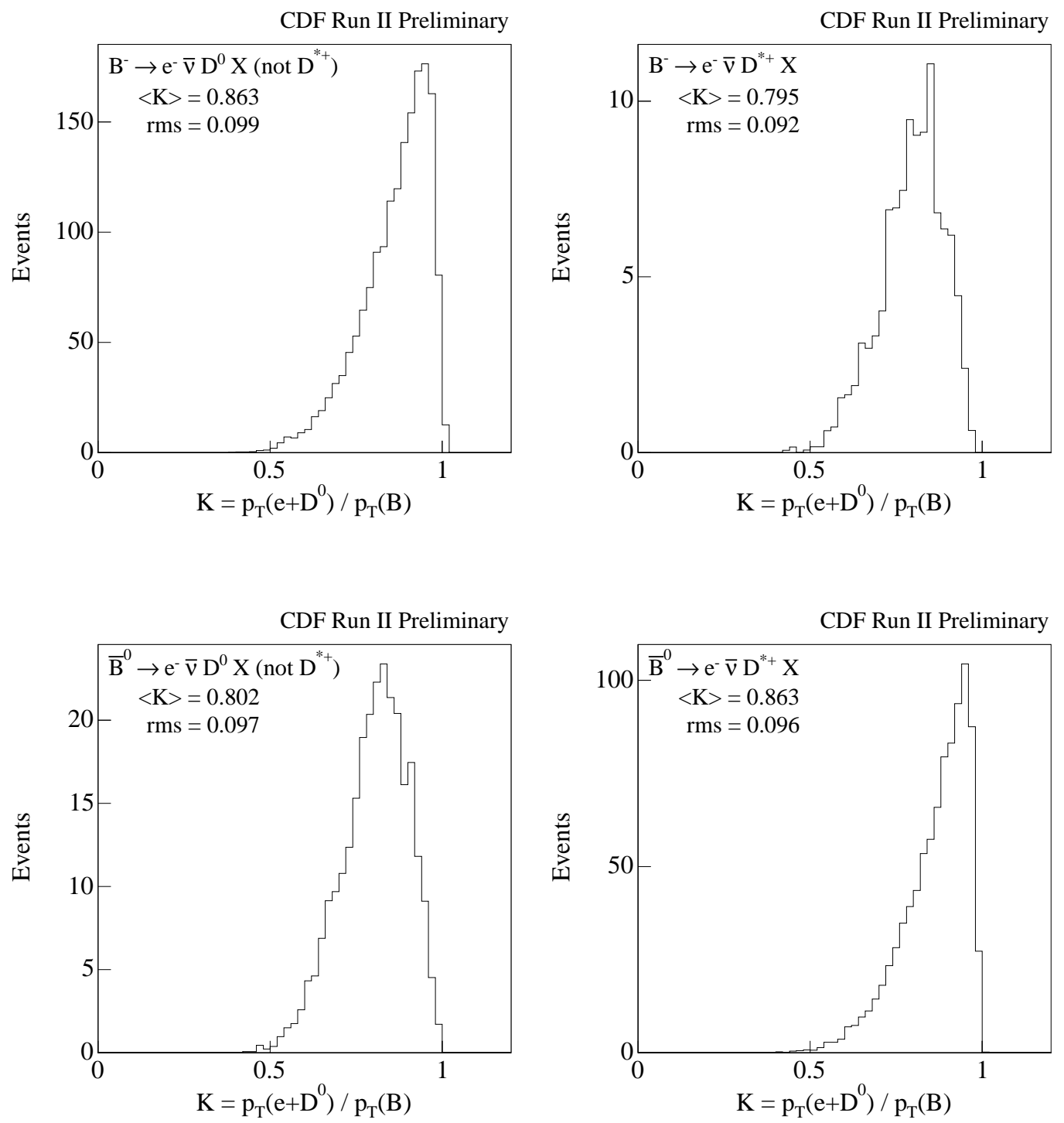

Figure 4.3: $K$ factor distributions for the electron dataset. 


\subsection{Combinatorial Background}

Now we turn to the combinatorial background modeling, a necessary step for background subtraction at the final $B^{-} / \bar{B}^{0}$ meson lifetime fit. We make use of the mass sideband events both for the $\ell^{-} D^{0}\left(D^{*+}\right.$ excluded $)$ and $\ell^{-} D^{*+}$ samples as background control sample. To describe the $c t^{*}$ shape of the combinatorial background, we define a background modeling function $\mathcal{F}_{\mathrm{BG}}$ as follows.

$$
\begin{aligned}
\mathcal{F}_{\mathrm{BG}}\left(c t^{*}\right)= & \left(1-f_{1+}-f_{+2}-f_{1-}-f_{2-}\right) G\left(c t^{*} ; \sigma\right) \\
& +f_{1+} \operatorname{Exp}\left(-c t^{*} ; \lambda_{1+}\right) \theta(x) \otimes G(\sigma) \\
& +f_{2+} \operatorname{Exp}\left(-c t^{*} ; \lambda_{2+}\right) \theta(x) \otimes G(\sigma) \\
& +f_{1-} \operatorname{Exp}\left(+c t^{*} ; \lambda_{1-}\right) \theta(-x) \otimes G(\sigma) \\
& +f_{2-} \operatorname{Exp}\left(+c t^{*} ; \lambda_{2-}\right) \theta(-x) \otimes G(\sigma),
\end{aligned}
$$

where $G$ is normalized Gaussian, and $\theta(x)$ is a step function (see Appendix A.1). The $\mathcal{F}_{\mathrm{BG}}$ has five components, central Gaussian and four smeared exponential tails both up and downward (two tails for each side). In the formula, $f$ and $\lambda$ denote the fraction and slope of each tail, and $\sigma$ shows resolution of the smearing. Subscripts,+- mean upper and lower tails. An exact analytic representation of $\mathcal{F}_{\mathrm{BG}}$ is described in Appendix A.2. We fit the sideband events

\begin{tabular}{|c|c|c|c|c|}
\hline & $\mu^{-} D^{0}$ & $\overline{\mu^{-} D^{*+}}$ & $e^{-} D^{0}$ & $e^{-} D^{*+}$ \\
\hline$\sigma$ & $16.5 \pm 5.6$ & $14.5 \pm 4.1$ & $14.4 \pm 6.2$ & $41.8 \pm 6.6$ \\
\hline$f_{1+}$ & $0.421 \pm 0.005$ & $0.581 \pm 0.018$ & $0.394 \pm 0.008$ & $0.609 \pm 0.016$ \\
\hline$\lambda_{1+}$ & $501.0 \pm 5.9$ & $537.3 \pm 14.4$ & $454.5 \pm 8.6$ & $503.8 \pm 15.8$ \\
\hline$f_{2+}$ & $0.220 \pm 0.006$ & $0.134 \pm 0.017$ & $0.230 \pm 0.009$ & $0.110 \pm 0.056$ \\
\hline$\lambda_{2+}$ & $67.8 \pm 2.6$ & $90.3 \pm 14.1$ & $58.1 \pm 3.1$ & $323.4 \pm 22.5$ \\
\hline$f_{1-}$ & $0.194 \pm 0.007$ & $0.150 \pm 0.019$ & $0.171 \pm 0.012$ & $0.171 \pm 0.044$ \\
\hline$\lambda_{1-}$ & $224.5 \pm 5.3$ & $231.6 \pm 16.1$ & $200.9 \pm 8.4$ & $164.5 \pm 18.9$ \\
\hline$f_{2-}$ & $0.172 \pm 0.008$ & $0.121 \pm 0.018$ & $0.206 \pm 0.013$ & $0.045 \pm 0.058$ \\
\hline$\lambda_{2-}$ & $55.8 \pm 2.8$ & $66.1 \pm 9.5$ & $54.8 \pm 3.6$ & $78.0 \pm 47.0$ \\
\hline fit probability & 0.001 & 0.372 & 0.001 & 0.184 \\
\hline
\end{tabular}
separately for the $\ell^{-} D^{0}\left(D^{*+}\right.$ excluded $)$ and $\ell^{-} D^{*+}$ samples using a unbinned likelihood method. The fit results are shown in Figure 4.4 for the muon and electron samples. Fit parameters are summarized in Table 4.1. These results are employed to describe background shape in the signal region in each sample.

Table 4.1: Fit results for the $c t^{*}$ shapes of the sideband events. Unit of $\sigma$ and $\lambda$ is $\mu \mathrm{m}$. 

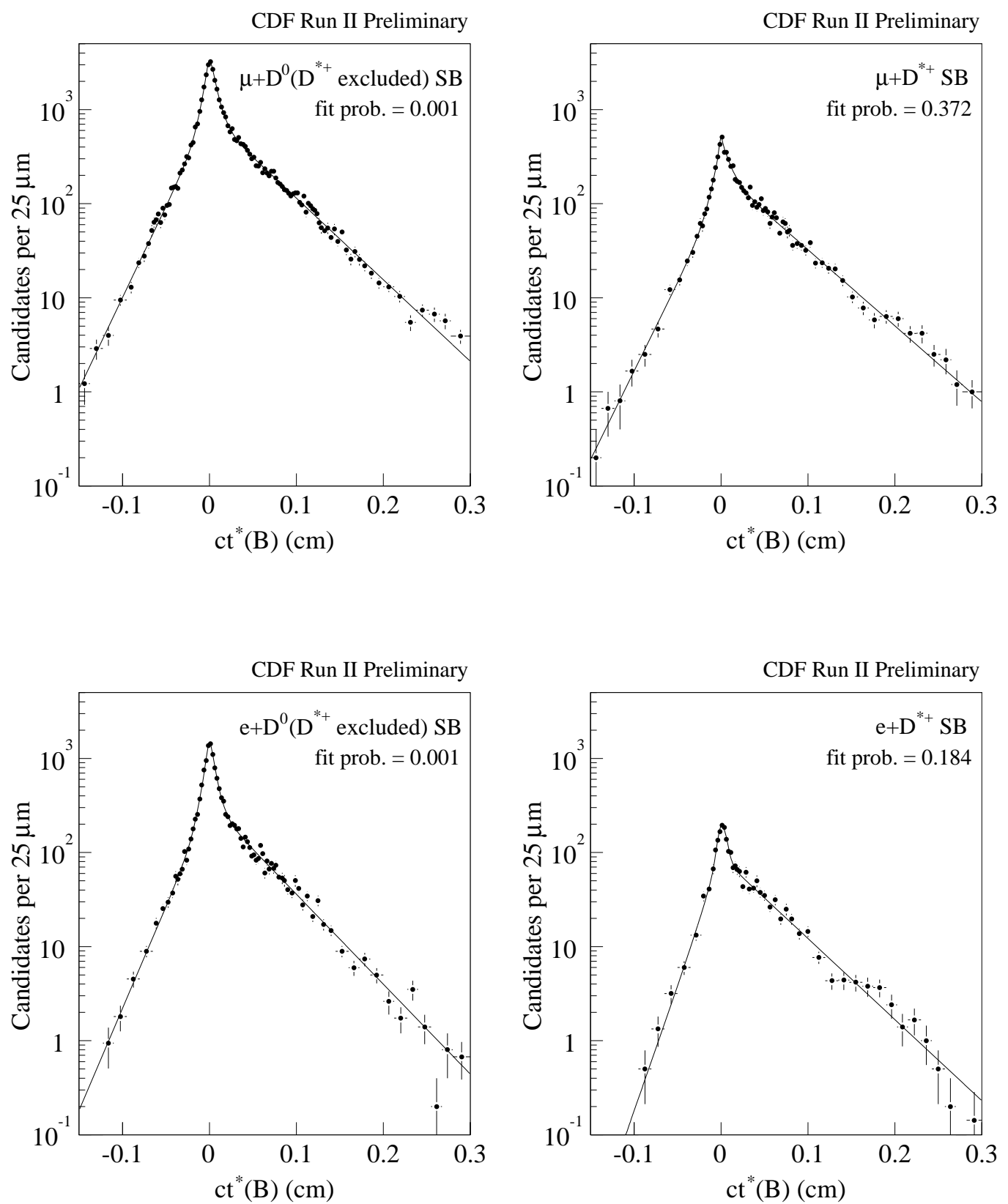

Figure 4.4: Fit results for $c t^{*}$ of the sideband events. These shapes represent $\mathcal{F}_{\mathrm{BG}}$. 


\subsection{Decay length resolution}

As written in Section 3.6, the $B^{-} / \bar{B}^{0}$ decay length $L_{B}$ and its uncertainty are obtained event by event in the vertex fit. From these numbers we calculate $c t^{*}$ and its uncertainty $\sigma_{c t^{*}}$. But it is known that $\sigma_{c t^{*}}$ obtained here is not exactly a right resolution of $c t^{*}[66]$. On an average, the real resolution of $c t^{*}$ is somewhat larger than $\sigma_{c t^{*}}$ obtained from the vertex fit. To correct this effect, we introduce a resolution scale factor each for the muon and electron datasets, and scale $\sigma_{c t^{*}}$ to be $s \sigma_{c t^{*}}$ in the lifetime fit.

The resolution scale factor $s$ is determined using special control sample. This sample is selected by requiring quite loose lepton ID cuts and $M\left(K^{-} \pi^{+}\right)$ region $1.74-1.79$, and $1.94-1.99 \mathrm{GeV} / c^{2}$. To loosen the lepton quality, we disable most of the offline lepton cuts : Some loose cuts by trigger and at a first selection stage are retained, because these cuts can not be disabled. These special samples contain plenty of fake leptons coming from the P.V., accordingly have an enhanced prompt peak and some little exponential tails in the $c t^{*}$ distribution. We extract the resolution scale factor $s$ assuming true $c t^{*}$ of the prompt peak component is 0 .

We define following modeling function to fit $c t^{*}$ of the prompt enhanced sample.

$$
\begin{aligned}
\mathcal{F}_{\text {prompt }}\left(c t^{*}, \sigma_{c t^{*}}\right) & =\left(1-f_{+}-f_{-}\right) G\left(c t^{*} ; s \sigma_{c t^{*}}\right) \\
& +f_{+} \operatorname{Exp}\left(-c t^{*} ; \lambda_{+}\right) \theta(x) \otimes G\left(s \sigma_{c t^{*}}\right) \\
& +f_{-} \operatorname{Exp}\left(+c t^{*} ; \lambda_{-}\right) \theta(-x) \otimes G\left(s \sigma_{c t^{*}}\right),
\end{aligned}
$$

An exact analytic formula of $\mathcal{F}_{\text {prompt }}$ is attached in Appendix A.3. The shape of $\mathcal{F}_{\text {prompt }}$ is similar with $\mathcal{F}_{\mathrm{BG}}$ described in Section 4.3 and A.2, but $\mathcal{F}_{\text {prompt }}$ takes both $c t^{*}$ and $\sigma_{c t^{*}}$ as event-by-event input parameters, and treats the scale factor $s$ as a fit parameter. And $\mathcal{F}_{\text {prompt }}$ has only two tails each for up and downward. Fitting results for the prompt enhanced samples are shown in Figure 4.5 both for the muon and electron datasets. From these fits, we determine the resolution scale factor to be $1.52 \pm 0.01$ for the muon dataset, and $1.45 \pm 0.02$ for the electron dataset. Table 4.2 summarizes all the fit parameters.

\begin{tabular}{cccccc}
\hline \hline Sample & $s$ & $f_{+}$ & $\lambda_{+}$ & $f_{-}$ & $\lambda_{-}$ \\
\hline$\mu^{-} D^{0}$ & $1.52 \pm 0.01$ & $0.346 \pm 0.003$ & $487.9 \pm 3.9$ & $0.138 \pm 0.003$ & $229.1 \pm 3.5$ \\
$e^{-} D^{0}$ & $1.45 \pm 0.02$ & $0.326 \pm 0.004$ & $464.5 \pm 6.7$ & $0.117 \pm 0.004$ & $206.0 \pm 6.1$ \\
\hline \hline
\end{tabular}

Table 4.2: Fitting results for the prompt enhanced samples on the resolution scale factor determination. Unit of $\lambda_{ \pm}$is $\mu \mathrm{m}$. 

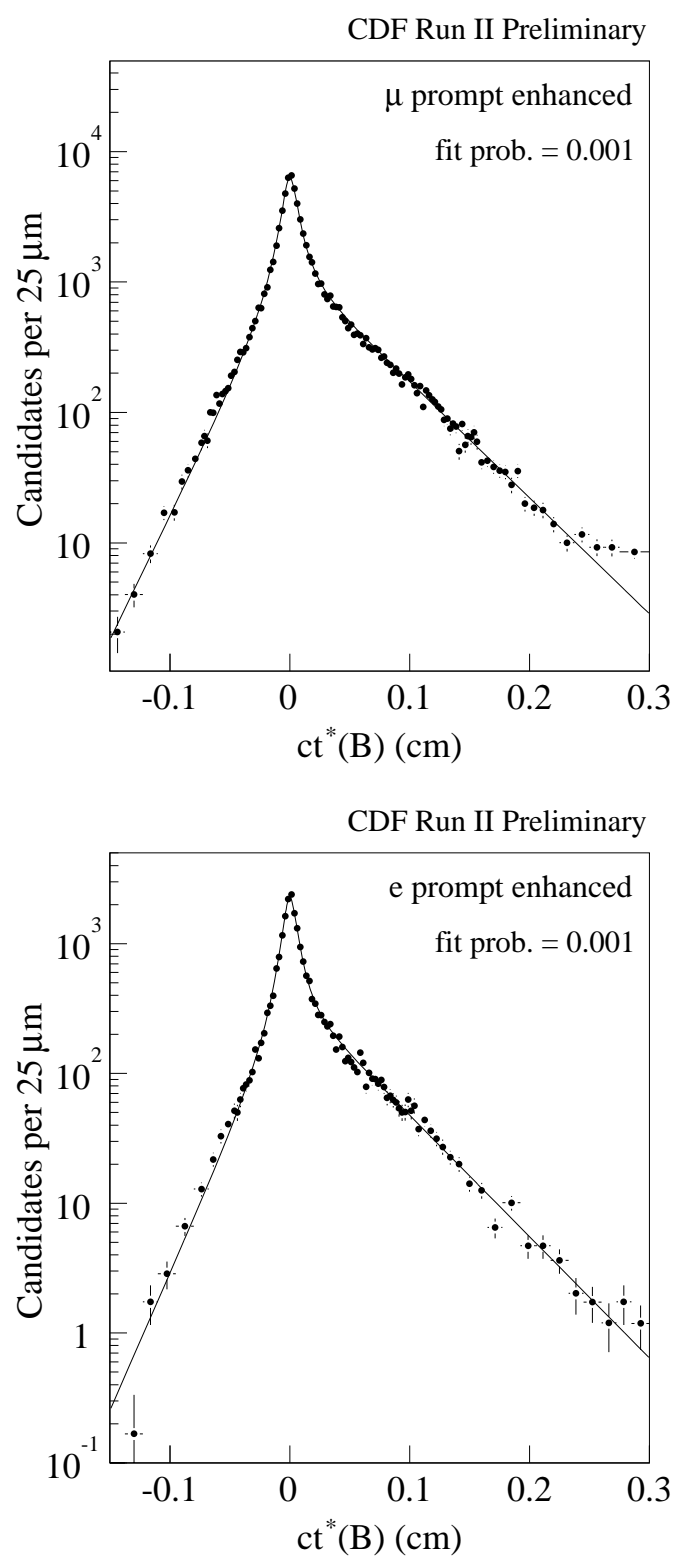

Figure 4.5: Fitting results for the prompt enhanced sample where we determine the resolution scale factor. Upper and lower plots show results for the muon and electron datasets respectively. 


\subsection{Physics backgrounds}

There are some physics processes which do not come from the semileptonic $B^{-} / \bar{B}^{0}$ decays but give $\ell^{-} D^{0}\left(\ell^{-} D^{*+}\right)$ final states with right charge correlation. Also, hadron $+D^{0}$ events sometimes fake to be $\ell^{-} D^{0}$. We define such events (either $\ell^{-}$is real or fake lepton) as the physics backgrounds. There are two possible sources which give the physics backgrounds.

1. $c, c \bar{c}$ created directly at the P.V.

2. $b, b \bar{b}$ event, but not $B^{-} / \bar{B}^{0} \rightarrow \ell^{-} D^{0} X$ decay

One evidence for the existence of the physics backgrounds is seen in the $\ell^{+} D^{0}$ events which have wrong charge correlation. Figure 4.6 shows the $M\left(K^{-} \pi^{+}\right)$ plots for $D^{0}$ (all) samples, but have wrong $\left(\ell^{+} D^{0}\right)$ charge correlation. As a result of the fit to the $M(K \pi)$ plots, we find $N_{D_{0}}=677.7 \pm 141.7$ for the muon WS sample, and $N_{D_{0}}=262.7 \pm 85.3$ for the electron WS sample. The ratio of $N_{D_{0}}$ in the WS and RS samples are $5.6 \pm 1.2 \%$ for the muon, and $5.0 \pm 1.6 \%$ for the electron. Since the WS events from the $B^{-} / \bar{B}^{0} \rightarrow \ell^{-} \bar{\nu} D^{0} X$ decays is at $0.4 \%$ level, it implies the presence of physics backgrounds.

Consequently we believe there are some physics backgrounds in our samples. However since they have track $+D^{0}$ final states (tracks are either real or fake leptons), it is very difficult to separate these backgrounds from the semileptonic $B^{-} / \bar{B}^{0}$ decay signals even if we utilize any kinematic quantities. (e.g. $M\left(\ell^{-} D^{0}\right), p_{T}, \Delta R$..) Besides, since there are expected to be some track$D^{0}$ charge correlation with the physics backgrounds, we can not make use of the WS events to estimate the fraction of the physics backgrounds. We try to estimate the fraction and shape of these backgrounds from the RS data as possible as we can, but in some parts we have to rely on $q \bar{q}$ Monte Carlo simulation. 

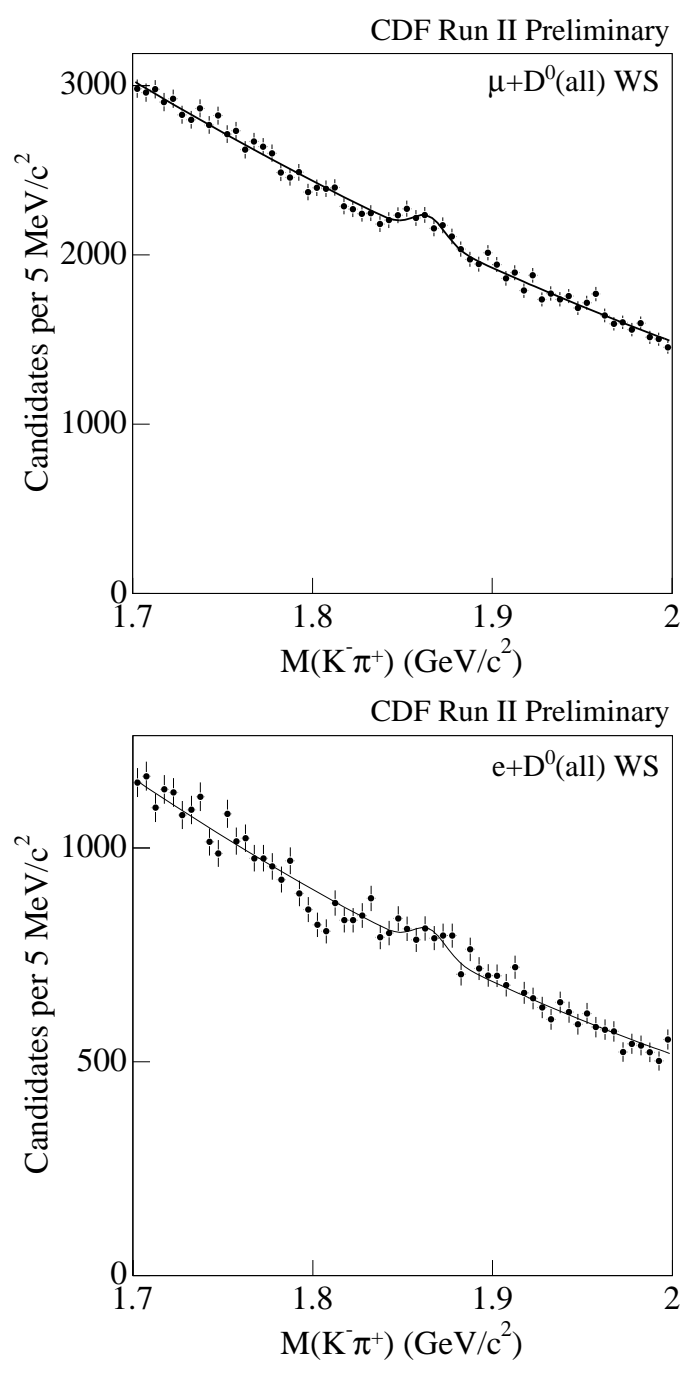

Figure 4.6: $M(K \pi)$ plots for $\ell^{+} D^{0}$ events, which have wrong charge correlation. 


\subsection{1 $c \bar{c}, b \bar{b}$ Monte Carlo samples}

Before beginning the discussion about each source of the physics backgrounds, we here describe about the $q \bar{q}$ Monte Carlo samples. These samples are created by Pythia [67] with configuration of generic $q \bar{q}$ generation. Events which contain charm and bottom quarks are filtered. All the mesons contained in the filtered events are decayed by the simulator. The charm and bottom quark events are split to form the separate $c \bar{c}, b \bar{b}$ Monte Carlo samples. No detector simulation is applied for these samples. Then we pick events which have a track $\left(p_{T}>8 \mathrm{GeV} / c\right)$ and $D^{0}$ with the right charge correlation, and decay $D^{0}$ into $K^{-} \pi^{+}$. Then we apply following kinematic cuts:

- $\mid \eta($ track $)|<0.7,| \eta(K, \pi) \mid<1.1$

- $p_{T}($ track $)>8 \mathrm{GeV} / c$

- $p_{T}(K)>1.5 \mathrm{GeV} / c, p_{T}(\pi)>0.5 \mathrm{GeV} / c$

- $\Delta R($ track $-K)<0.6, \Delta R($ track $-\pi)<0.7$

These $c \bar{c}, b \bar{b}$ Monte Carlo samples are used later in this section.

\subsubsection{Prompt charm background}

First possible source of the physics backgrounds come from charm quark events (directly generated $c$ or $c \bar{c}$ ). In this type of events, real $D^{0}\left(D^{*+}\right)$ meson is created at the P.V. A track that has $p_{T}>8 \mathrm{GeV} / c$, either a real or fake lepton, is also generated at the P.V. or from $\bar{c}$ quark decay. Such prompt charm events are expected to have almost zero $c t^{*}$, eventually give relatively large effects to the $B^{-} / \bar{B}^{0}$ meson lifetime measurements. Thus they must be subtracted properly. However it is difficult to know how much the prompt charm background events exist in the data samples using any kinematic quantities. So we try to make use of the decay length information.

To estimate the fraction of the charm background from the RS data, we define following quantity.

$$
c t_{2}=\frac{L_{x y}\left(D^{0}-P . V .\right)}{p_{T}\left(K^{-} \pi^{+}\right)} M\left(K^{-} \pi^{+}\right)
$$

In the prompt charm event, the $D^{0}$ meson is always generated at the P.V. and the distribution of the quantity $c_{2}$ is an exponential distribution with the $D^{0}$ meson lifetime, $c \tau\left(D^{0}\right)=123 \mu \mathrm{m}$. For the events which come from the $B$ meson (both semileptonic $B^{-} / \bar{B}^{0}$ decays or bottom background), the lifetime of $c_{2}$ is much longer. Based on this fact we estimate the prompt charm background 
fraction by fitting the $c_{2}$ distribution with templates of the charm and bottom components.

At first we fit the $c_{2}$ distributions of the sideband events to get the combinatorial background shape. We use the same function with the $c t^{*}$ background function $\mathcal{F}_{\mathrm{BG}}$ (see Section 4.3). The sideband fit results are shown in Figure 4.7 for both the muon and electron samples. Then we prepare for following two templates.

1. charm component function $\left(F_{c}\right)$

... described by a decay exponential with lifetime $c \tau=123 \mu \mathrm{m}$, smeared with resolution function.

2. $B$ component function $\left(F_{B}\right)$

... obtained from $B^{-} / \bar{B}^{0}$ Monte Carlo sample which is used for $K$ factor calculation.

The $F_{c}$ is an analytic function defined as following formula.

$$
F_{c}\left(c t_{2}\right)=\operatorname{Exp}\left(-c t_{2} ; 123 \mu \mathrm{m}\right) \otimes G\left(s_{2} \sigma_{c t_{2}}\right),
$$

where the $\operatorname{Exp}(-x ; a)$ denotes a normalized decay exponential function with lifetime $a$, and $\sigma_{c t_{2}}$ is an uncertainty of $c t_{2}$ obtained at the vertex fit, and $s_{2}$ is the resolution scale factor for $\sigma_{c t_{2}}$ (discussed later in this subsection).

On the other hand $F_{B}$ is a histogram created from the $B^{-} / \bar{B}^{0}$ Monte Carlo samples, because we can not write an analytic formula of the $c t_{2}$ distribution for the $B$ component.

Before the fit we need to know the $c_{2}$ resolution to smear both templates. We obtain the real resolution for $c_{2}$ using the same method discussed in Section 4.4. With the prompt enhanced sample, we determine the resolution scale factor for $c t_{2}$. And from the $\sigma_{c t_{2}}$ distribution, mean value of $\sigma_{c t_{2}}\left(=\left\langle\sigma_{c t_{2}}\right\rangle\right)$ is obtained. We find $s_{2}=1.34 \pm 0.01$ and $\left\langle\sigma_{c t_{2}}\right\rangle=30.1 \mu \mathrm{m}$ for the muon sample, and $s=1.32 \pm 0.01,\left\langle\sigma_{c t_{2}}\right\rangle=33.0 \mu \mathrm{m}$ for the electron dataset.

For the template of the $B$ component, we take $c t_{2}$ from generator-level information which includes resolution effect, and smear it with the measured $c t_{2}$ resolution $\left(=s_{2} \cdot\left\langle\sigma_{c t_{2}}\right\rangle\right)$. For the charm template, we use $s_{2}$ directly as a parameter of $F_{c}$.

Now we fit the prompt charm fraction $f_{c}$ using the templates and background function. At the fit the $L_{x y}$ cut is disabled. The fits are performed with unbinned likelihood method, separately for the muon and electron, $D^{0}$ ( $D^{*+}$ excluded) and $D^{*+}$ samples. The probability density function is defined as follows.

$$
F=f_{\text {sig }}\left\{f_{c} F_{c}+\left(1-f_{c}\right) F_{B}\right\}+\left(1-f_{\text {sig }}\right) F_{\mathrm{BG}}
$$


Here we define the prompt charm background fraction as

$$
f_{c}=\frac{N_{c}}{N_{D^{0}}\left(\text { or } N_{D^{*+}}\right)},
$$

where $N_{c}$ is the number of prompt charm background events and $N_{D^{0}}\left(N_{D^{*+}}\right)$ is the number of $\ell^{-} D^{0}\left(\ell^{-} D^{*+}\right)$ signals obtained from the mass distribution. After the fit we correct the obtained $f_{c}$ values considering the $L_{x y}>0$ cut. Fit results are shown in Figure 4.8 and summarized in Table 4.3. For the muon dataset, we find $f_{c}=5.7 \pm 1.2 \%$ for the $\mu^{-} D^{0}\left(D^{*+}\right.$ excluded $)$ sample, and $5.8 \pm 1.3 \%$ for the $\mu^{-} D^{*+}$ sample. For the electron dataset, the corresponding numbers are much smaller. The reason is still not fully understood, but we think some of the electron ID cuts might have suppressed the charm background. Since negative $f_{c}$ is not physical, we interpret $f_{c}$ for electron dataset as $0.0_{-0.0}^{+2.0} \%$ for $e^{-} D^{0}\left(D^{*+}\right.$ excluded), and $0.3_{-0.3}^{+1.9} \%$ for $e^{-} D^{*+}$.

\begin{tabular}{lccc}
\hline \hline Sample & $f_{c}$ (no $L_{x y}$ cut) & $f_{c}\left(L_{x y}>0\right)$ & fit probability \\
\hline$\mu^{-} D^{0}$ & $6.3 \pm 1.3 \%$ & $5.7 \pm 1.2 \%$ & 0.0132 \\
$\mu^{-} D^{*+}$ & $6.4 \pm 1.4 \%$ & $5.8 \pm 1.3 \%$ & 0.1066 \\
$e^{-} D^{0}$ & $-0.5 \pm 2.0 \%$ & $-0.5 \pm 2.0 \%$ & 0.0144 \\
$e^{-} D^{*+}$ & $0.3 \pm 2.1 \%$ & $0.3 \pm 1.9 \%$ & 0.3172 \\
\hline \hline
\end{tabular}

Table 4.3: Summary of the charm background fraction in each sample.

Secondly we determine the $c t^{*}$ shapes of the charm background. The charm background events can be classified into following two types.

1. Events which have $c t^{*}=0 \ldots$ the "lepton" is a track from P.V. The track is either from QCD process, $D$ meson fragmentation, unstable $D$ hadron (e.g. $D^{* *+} \rightarrow D^{0} \pi^{+}$).

2. Events which have $c t^{*} \neq 0 \ldots$ from $c \bar{c}, c \rightarrow D^{0}\left(D^{*+}\right), \bar{c} \rightarrow \bar{D}, \bar{D} \rightarrow$ "lepton" track $+X . D^{0}$ is from P.V., the track is from pseudo-scalar charm hadron $\bar{D}\left(\bar{D}^{0}, D^{-}, \bar{\Lambda}_{c} \ldots\right)$.

The $c t^{*}$ shape of the type- 1 events is a Gaussian centered at zero, and $\sigma$ is given by the $c t^{*}$ resolution, defined as following formula.

$$
\mathcal{F}_{c}^{\text {zero }}=G\left(c t^{*} ; s \sigma_{c t^{*}}\right),
$$

where $s$ is the resolution scale factor measured in Section 4.4, and $\sigma_{c t^{*}}$ is the $c t^{*}$ uncertainty obtained at the vertex fit event by event. 
For the type-2 events, the $c t^{*}$ shape is not so simple. We use the $c \bar{c}$ Monte Carlo samples to get the shape. From the $c \bar{c}$ Monte Carlo samples we select $\ell^{-} D^{0}$ events which have non-zero $c t^{*}$. Figure 4.9 shows the $c t^{*}$ shape of the $c t^{*} \neq 0$ events. We assume the $c t^{*}$ resolution is $70 \mu \mathrm{m}$ to make the $\mathcal{F}_{c}^{\text {non-zero }}$ distribution. We fit this $c t^{*}$ distribution with a following function $\mathcal{F}_{c}^{\text {non-zero }}$ which has a central Gaussian and two exponential tails.

$$
\begin{aligned}
\mathcal{F}_{c}^{\text {non-zero }} & =\left(1-f_{+}-f_{-}\right) G\left(c t^{*} ; \sigma\right) \\
& +f_{+} \operatorname{Exp}\left(-c t^{*} ; \lambda_{+}\right) \theta(x) \otimes G(\sigma) \\
& +f_{-} \operatorname{Exp}\left(+c t^{*} ; \lambda_{-}\right) \theta(-x) \otimes G(\sigma)
\end{aligned}
$$

Parameters of $\mathcal{F}_{c}^{\text {non-zero }}$ are summarized in Table 4.4. The $c t^{*}$ shape of the charm background events in the real data is expected to be a combination of both $\mathcal{F}_{c}^{\text {zero }}$ and $\mathcal{F}_{c}^{\text {non-zero }}$ shapes. But the fraction of both shapes is hardly determined, because it depends on ratio of the single- $c$ and $c \bar{c}$ events, fake rate of the lepton, and so on. So we take $\mathcal{F}_{c}^{\text {zero }}$ as the template of the charm background at the final lifetime fit. The $\mathcal{F}_{c}^{\text {non-zero }}$ is used for evaluation of the systematic uncertainty.

\begin{tabular}{lccccc}
\hline \hline Type & $\sigma$ & $f_{+}$ & $\lambda_{+}$ & $f_{-}$ & $\lambda_{-}$ \\
\hline$c t^{*} \neq 0$ & $93.2 \pm 6.4$ & $0.126 \pm 0.025$ & $800.0 \pm 193.5$ & $0.098 \pm 0.023$ & $975.4 \pm 579.5$ \\
\hline \hline
\end{tabular}

Table 4.4: The $\mathcal{F}_{c}^{\text {non-zero }}$ parameters. Unit of $\sigma$ and $\lambda_{ \pm}$is $\mu \mathrm{m}$. 

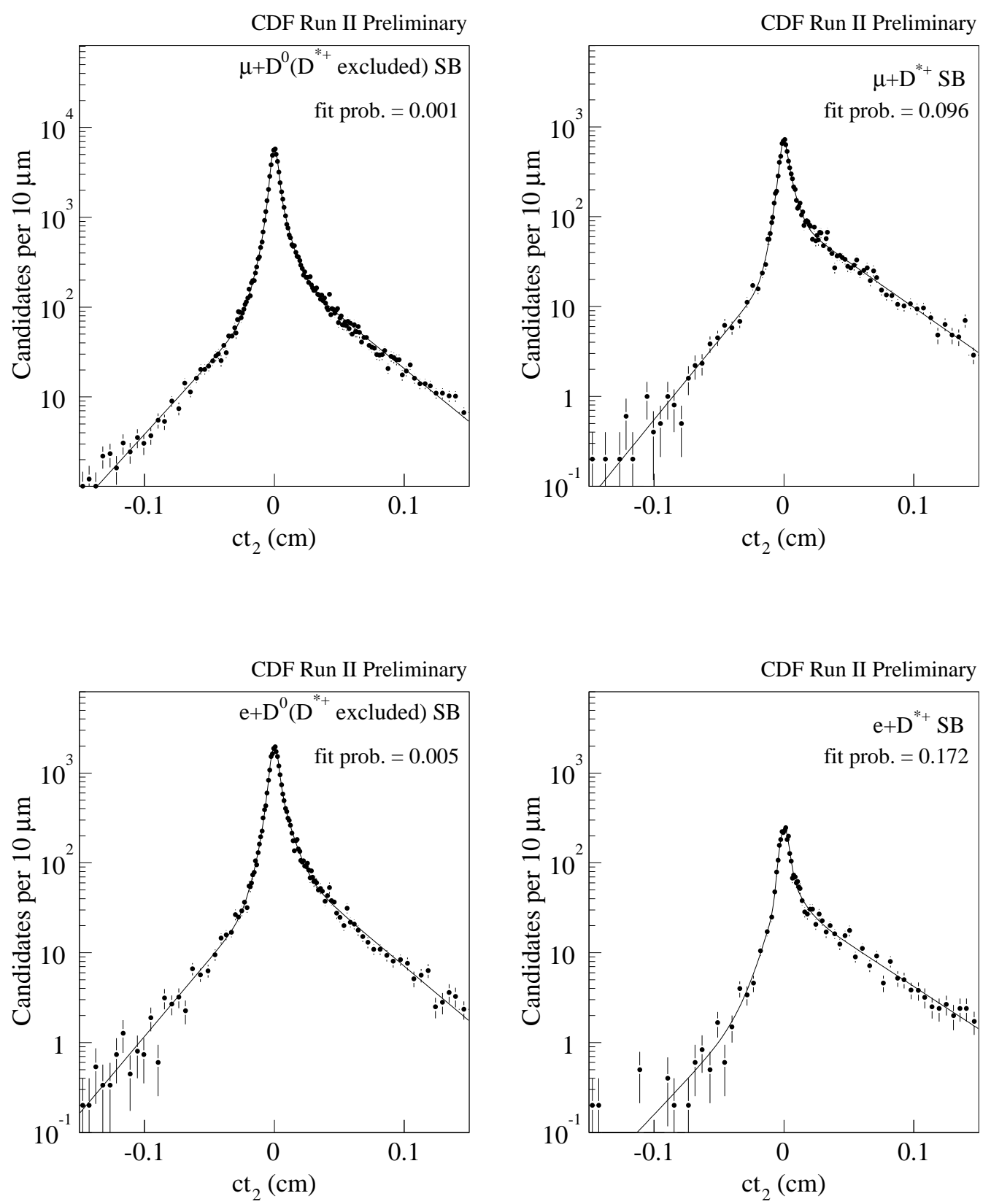

Figure 4.7: Fit for $c t_{2}$ of the sideband events. These fitting results are taken as background shapes in the $f_{c}$ fits. 

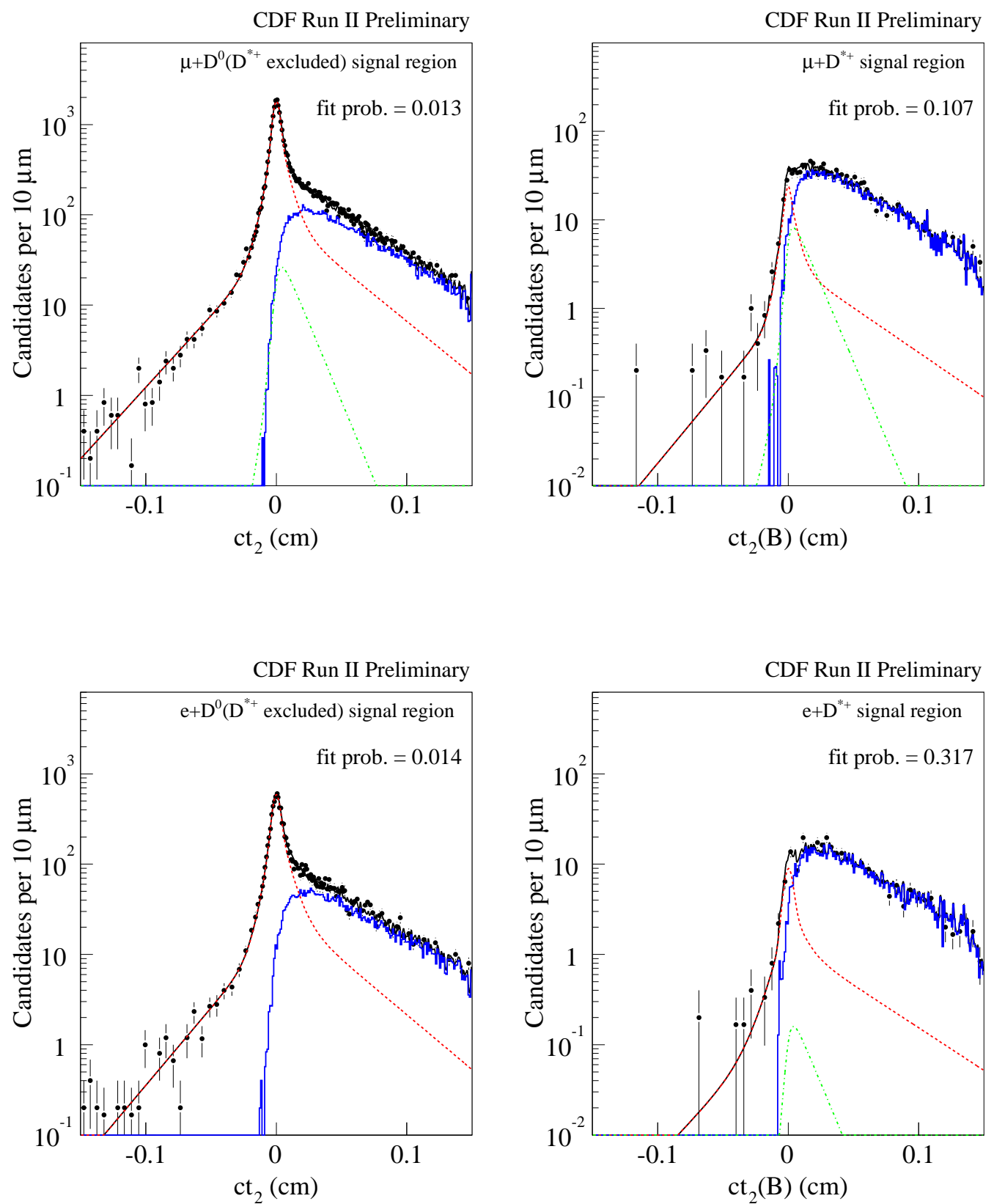

Figure 4.8: Fitting result for the $f_{c}$ determination. In each plot green and blue curves show charm and $B$ templates respectively. Red curves describe combinatorial background shapes. 


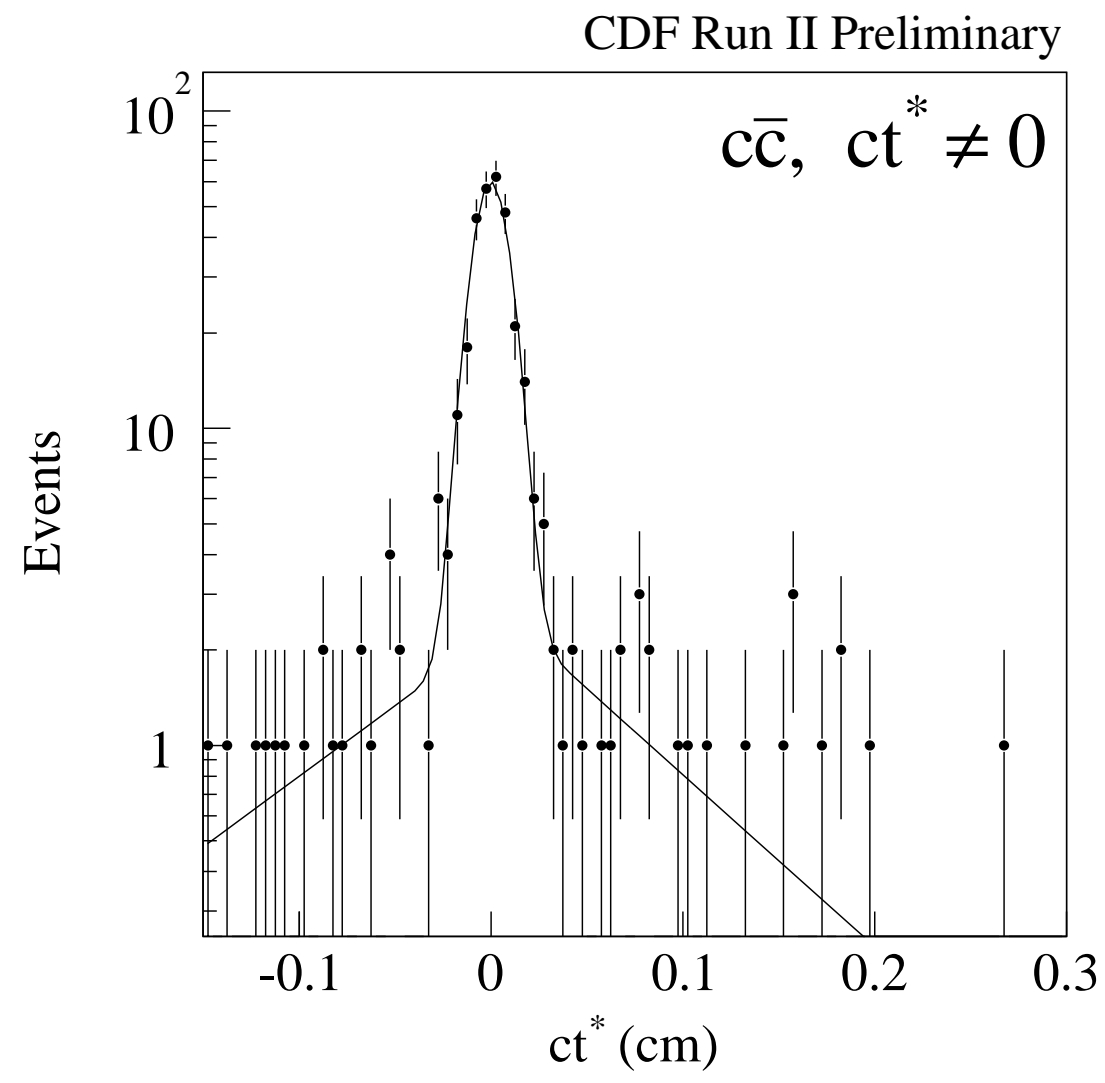

Figure 4.9: $F_{c}$ shape for $c t^{*} \neq 0$ events in the charm background, obtained from $c \bar{c}$ Monte Carlo sample. 


\subsubsection{Bottom background}

There are some events which originate from the $\bar{B}$ mesons $\left(\bar{B}^{0}, B^{-}, \bar{B}_{s}^{0}\right)$ and provide the $\ell^{-} D^{0}$ final state, but not through the semileptonic decays $\left(\bar{B}^{0} / B^{-} \rightarrow \ell^{-} \bar{\nu} D^{0} X, \ell^{-}=e^{-}, \mu^{-}\right)$. Here we call these events as non-semileptonic $\ell^{-} D^{0}$ events. There are also hadron $+D^{0}$ events where hadrons fake to be leptons. We define both the non-semileptonic $\ell^{-} D^{0}$ and hadron $+D^{0}$ events as the bottom background.

Since it is almost impossible to distinguish the bottom background in the data sample, we need to make use of the $b \bar{b}$ Monte Carlo samples to estimate the effect of them. Fortunately branching fractions of various $B$ meson decays are measured by many experiments, and these numbers are implemented in the simulation. So we can extract the bottom background fraction from the $b \bar{b}$ Monte Carlo samples relatively reliably. This is a large difference with the charm background case, where the $c \bar{c}$ Monte Carlo is not reliable to extract the prompt charm fraction because we do not have reliable estimation of the number of prompt charm events against the semileptonic $B^{-} / \bar{B}^{0}$ signals. Also the bottom background events are expected to have relatively long lifetime which is compatible to the $B$ meson lifetime, thus effect to the lifetime fit will be relatively smaller than the charm background.

From the $b \bar{b}$ Monte Carlo samples we pick events which have RS track + $D^{0}\left(D^{*+}\right)$ final states. Then we count the number of events in each decay process. Table 4.5 summarizes the decay processes of the $b \bar{b}$ Monte Carlo samples after the kinematic cuts. There are about $4 \%$ of non-semileptonic $\ell^{-} D^{0}$ events against the semileptonic $B^{-} / \bar{B}^{0}$ decay signals. If we assume the fake lepton ID efficiency for hadrons is about $1 \%$, the amount of residual hadron $+D^{0}$ in the bottom background is order of $0.5 \%$ of the semileptonic $B^{-} / \bar{B}^{0}$ signals, which is much smaller than the non-semileptonic $\ell^{-} D^{0}$ events.

We categorize the $b \bar{b}$ Monte Carlo sample into four subsets, $\ell^{-} D^{0}, \ell^{-} D^{*+}$ (both non-semileptonic), $h^{-} D^{0}$ and $h^{-} D^{*+}$. Figure 4.10 shows $c t^{*}$ shapes of each subset. To model these shapes we use following function $\mathcal{F}_{b}$.

$$
\begin{aligned}
\mathcal{F}_{b} & =\left(1-f_{+}-f_{-}\right) G\left(c t^{*}-\alpha ; \sigma\right) \\
& +f_{+} \operatorname{Exp}\left(-c t^{*} ; \lambda_{+}\right) \theta(x) \otimes G(\sigma) \\
& +f_{-} \operatorname{Exp}\left(+c t^{*} ; \lambda_{-}\right) \theta(-x) \otimes G(\sigma)
\end{aligned}
$$

Table 4.6 summarizes fit results of the $\mathcal{F}_{b}$ parameters for each subset. For the $\ell^{-} D^{*+}$ events, due to small statistics, we need to remove a negative tail to make the fit converged.

Since the hadron $+D^{0}$ contribution is expected to be small, and we can hardly measure the lepton fake rate, we take only the non-semileptonic $\ell^{-} D^{0}$ results as bottom background. To cover an incomplete knowledge about the 
residual $h^{-} D^{0}$ fraction, we assign additional uncertainty of $+0.5 \%$ to $f_{b}$. This uncertainty is used at the evaluation of systematic uncertainties described in Section 4.8. Also, the $c t^{*}$ shape of the hadron $+D^{0}$ events is used for systematics evaluation.

From the Table 4.5, we find

$$
f_{b}^{\prime}=\frac{N_{b}}{N_{\mathrm{sl}}}= \begin{cases}4.2 \pm 0.3 \% & \text { for } \ell^{-} D^{0}\left(D^{*+} \text { excluded }\right) \text { sample } \\ 4.0 \pm 0.4 \% & \text { for } \ell^{-} D^{*+} \text { sample }\end{cases}
$$

where $N_{b}$ is number of the non-semileptonic $\ell^{-} D^{0}$ background events, and $N_{\mathrm{Sl}}$ is the number of the semileptonic $B^{-} / \bar{B}^{0}$ decay signals. From $f_{b}^{\prime}$ and $f_{c}$ we extract the bottom background fraction $f_{b}$ in each sample.

$f_{b}=\frac{N_{b}}{N_{\mathrm{Sl}}+N_{c}+N_{b}}=\frac{f_{b}^{\prime}}{1+f_{b}^{\prime}}\left(1-f_{c}\right)= \begin{cases}3.8 \pm 0.3 \% & \text { for } \mu+D^{0}\left(D^{*+} \text { excluded }\right) \text { sample } \\ 3.6 \pm 0.4 \% & \text { for } \mu+D^{*+} \text { sample } \\ 4.0 \pm 0.3 \% & \text { for } e+D^{0}\left(D^{*+} \text { excluded }\right) \text { sample } \\ 3.8 \pm 0.4 \% & \text { for } e+D^{*+} \text { sample }\end{cases}$

\begin{tabular}{lll}
\hline \hline process & $\ell^{-} D^{0}\left(D^{*+}\right.$ excluded $)$ & $\ell^{-} D^{*+}$ \\
\hline Semileptonic $\boldsymbol{\ell}^{-} \boldsymbol{D}^{\mathbf{0}}$ & $\mathbf{5 2 2 6}$ & $\mathbf{2 7 0 2}$ \\
non-semileptonic $\boldsymbol{\ell}^{-} \boldsymbol{D}^{\mathbf{0}}$ & $\mathbf{2 1 7}$ & $\mathbf{1 0 8}$ \\
$\bar{B}_{s}^{0} \rightarrow \ell^{-} \bar{\nu} D_{s}^{* *+}, D_{s}^{* *+} \rightarrow D^{0} X$ & 84 & 50 \\
$\bar{B} \rightarrow \tau^{-} \bar{\nu} D^{0}, \tau^{-} \rightarrow \ell^{-} \bar{\nu}$ & 52 & 27 \\
$\bar{B} \rightarrow D^{0} D_{s}^{-} X, D_{s}^{-} \rightarrow \ell^{-} X^{\prime}$ & 46 & 8 \\
$\bar{B} \rightarrow D^{0} D_{s}^{-} X, D_{s}^{-} \rightarrow \tau^{-} X^{\prime}, \tau^{-} \rightarrow \ell^{-} X^{\prime \prime}$ & 18 & 5 \\
$\bar{B} \rightarrow D \bar{D} X \rightarrow \ell^{-} D^{0} X^{\prime}$ & 7 & 6 \\
$b \bar{b}, b \rightarrow D^{0} X, \bar{b} \rightarrow \ell^{+} X^{\prime}$, & 6 & 8 \\
$(b$ or $\bar{b}$ oscillates and give RS) & 2 & 1 \\
$b \bar{b}, b \rightarrow D^{0} X, \bar{b} \rightarrow \bar{c} \rightarrow \ell^{-} X^{\prime}$ & 2 & 3 \\
others & $\mathbf{3 3 7 1}$ & $\mathbf{2 1 3 8}$ \\
hadron $+\boldsymbol{D}^{\mathbf{0}}$ & 3308 & 2117 \\
single $b \rightarrow h^{-} D^{0}$ & 63 & 21 \\
$b \bar{b} \rightarrow h^{-} D^{0}$ &
\end{tabular}

Table 4.5: A summary of the $b \bar{b}$ Monte Carlo events with RS track $+D^{0}$ final states after kinematic cuts. $\ell^{-}$is $\mu^{-}$or $e^{-}$, and $\bar{B}$ denotes either $B^{-}$or $\bar{B}^{0}$. 


\begin{tabular}{lcccccc}
\hline \hline Type & $\sigma$ & $f_{+}$ & $\lambda_{+}$ & $f_{-}$ & $\lambda_{-}$ & $\alpha$ \\
\hline$\ell^{-} D^{0}$ & $49.7 \pm 54.6$ & $0.914 \pm 0.035$ & $770.8 \pm 73.7$ & $0.042 \pm 0.019$ & $396.8 \pm 213.3$ & $-0.5 \pm 58.4$ \\
$\ell^{-} D^{*+}$ & $185.6 \pm 35.1$ & $0.719 \pm 0.150$ & $993.5 \pm 276.8$ & 0 (fixed) & - & $227.3 \pm 64.0$ \\
$h^{-} D^{0}$ & $79.3 \pm 5.4$ & $0.913 \pm 0.016$ & $702.7 \pm 192.2$ & $0.018 \pm 0.003$ & $504.6 \pm 122.5$ & $108.7 \pm 16.1$ \\
$h^{-} D^{*+}$ & $78.1 \pm 5.9$ & $0.970 \pm 0.018$ & $693.7 \pm 22.1$ & $0.005 \pm 0.002$ & $1352.9 \pm 1942.5$ & $105.0 \pm 41.1$ \\
\hline \hline
\end{tabular}

Table 4.6: Fit results for four types of bottom background. $\ell^{-} D^{0}$ and $\ell^{-} D^{*+}$ denote the non-semileptonic $B$ events here. Unit of $\sigma, \lambda$ and $\alpha$ is $\mu \mathrm{m}$.
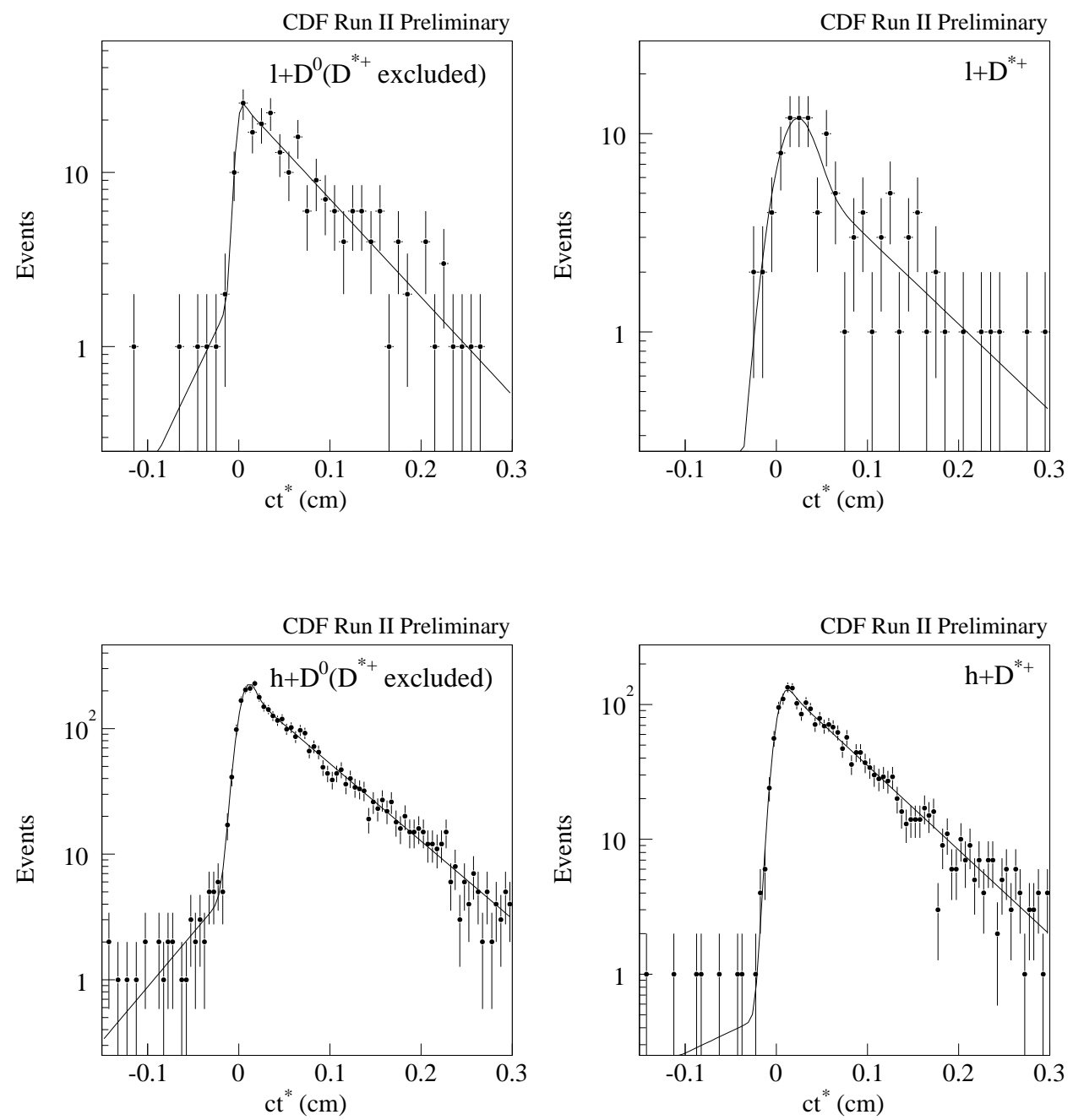

Figure 4.10: $c t^{*}$ shapes of the bottom background events obtained from the $b \bar{b}$ Monte Carlo samples. 


\subsection{Sample Composition}

To extract the $B^{-}$and $\bar{B}^{0}$ meson lifetimes, we need to know the mixture of the $B^{-}$and $\bar{B}^{0}$ components each in the $\ell^{-} D^{0}\left(D^{*+}\right.$ excluded) and $\ell^{-} D^{*+}$ sample. The $\ell^{-} D^{0}$ sample is dominated by $B^{-}$, and the $\ell^{-} D^{*+}$ sample is dominated by $\bar{B}^{0}$ component. If there are no $\bar{B} \rightarrow \ell^{-} \bar{\nu} D^{* *}$ decays, all events in the $\ell^{-} D^{0}$ sample are from the $B^{-}$mesons, and the $\ell^{-} D^{*+}$ events are all from $\bar{B}^{0}$ mesons (assuming a perfect $\pi_{s}^{+}$finding efficiency). Since $\bar{B}$ can decay into $\ell^{-} \bar{\nu} D^{* *}$, there is a little dilution. In addition to it, another cross talks come from inefficiency of the $\pi_{s}$ track reconstruction. In this section we estimate the sample composition considering these facts.

\subsection{1 $\pi_{s}^{+}$reconstruction efficiency}

Since the decay $D^{*+} \rightarrow D^{0} \pi_{s}^{+}$has a low $Q$ value $(39 \mathrm{MeV})$, the $\pi_{s}^{+}$tracks normally have small transverse momentum. In such low transverse momentum region COT tracking efficiency declines from unity. Due to this inefficiency, we may count some of the $\ell^{-} D^{*+}$ events as the $\ell^{-} D^{0}\left(D^{*+}\right.$ excluded) sample. Eventually it becomes a source of the dilution. We estimate the slow pion reconstruction efficiency $\epsilon\left(\pi_{s}\right)$ using the ratio of $\ell^{-} D^{*+}$ and $\ell^{-} D^{0}$ ( $D^{*+}$ excluded) yields. Figures $4.11-4.18$ show $M\left(K^{-} \pi^{+}\right)$and $\Delta M$ for $D^{0}$ ( $D^{*+}$ excluded) and $D^{*+}$ samples in each $D^{0}$ transverse momentum ranges both for the muon and electron datasets. From these distributions we calculate the ratio of $\ell^{-} D^{*+}$ and $\ell^{-} D^{0}\left(D^{*+}\right.$ excluded) yields. Table 4.7 summarizes the $D^{0}$ and $D^{*+}$ signal yields in each $p_{T}\left(D^{0}\right)$ region and Figure 4.19 shows the ratio of yields against $D^{0}$ transverse momentum. In the region $p_{T}\left(D^{0}\right)>8 \mathrm{GeV} / c$ there are plateau regions where the $\pi_{s}^{+}$reconstruction efficiency is almost $100 \%$. And it starts to decrease around $p_{T}\left(D^{0}\right) \sim 8 \mathrm{GeV} / c$. Figure 4.20 shows the transverse momentum distributions for slow pion and $D^{0}$ for $\mu^{-} D^{*+}$ signal region. Correlation of these momenta is also shown.

First we determine $\epsilon\left(\pi_{s}\right)$ at the plateau. Average of the ratio of yield is $0.327 \pm 0.010$ in $p_{T}\left(D^{0}\right)>8 \mathrm{GeV} / c$. According to other study [65], COT tracking efficiency for $p_{T}>1.5 \mathrm{GeV} / c$ track is $99.6 \pm 0.02_{-0.91}^{+0.34} \%$. We take this number as the $\pi_{s}^{+}$reconstruction efficiency at the plateau level. And we find

$$
\epsilon\left(\pi_{s}\right)_{\text {plateau }}=0.996 \pm 0.032
$$

Then we split the data sample into two regions according to the transverse momentum of $D^{0}$ meson each in the muon and electron datasets. The first region is $p_{T}\left(D^{0}\right)<8 \mathrm{GeV} / c$, where $\epsilon\left(\pi_{s}\right)$ drops from unity. The second is the plateau region in $p_{T}\left(D^{0}\right)>8 \mathrm{GeV} / c$. The measured $\epsilon\left(\pi_{s}\right)$ in each region are summarized in Table 4.8. 


\begin{tabular}{|c|c|c|c|c|}
\hline Dataset & $\bar{p}_{T}\left(D^{0}\right)$ region $(\mathrm{GeV} / \mathrm{c})$ & $N_{D^{*+}}$ & $N_{D^{0}}$ & 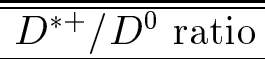 \\
\hline \multirow{7}{*}{ Muon } & $<4$ & $42.1 \pm 8.3$ & $392.1 \pm 35.9$ & $0.107 \pm 0.023$ \\
\hline & $4-5$ & $186.7 \pm 16.9$ & $1115.2 \pm 53.3$ & $0.167 \pm 0.017$ \\
\hline & $5-6$ & $305.5 \pm 20.8$ & $1131.8 \pm 56.2$ & $0.270 \pm 0.023$ \\
\hline & $6-8$ & $681.0 \pm 29.1$ & $2261.7 \pm 76.1$ & $0.301 \pm 0.016$ \\
\hline & $8-10$ & $529.5 \pm 25.1$ & $1609.4 \pm 61.9$ & $0.329 \pm 0.020$ \\
\hline & $10-12$ & $327.0 \pm 19.3$ & $1034.4 \pm 49.9$ & $0.316 \pm 0.024$ \\
\hline & $>12$ & $600.4 \pm 25.8$ & $1827.4 \pm 68.7$ & $0.329 \pm 0.019$ \\
\hline \multirow{7}{*}{ Electron } & $<4$ & $39.3 \pm 7.3$ & $305.3 \pm 31.0$ & $0.107 \pm 0.023$ \\
\hline & $4-5$ & $148.2 \pm 13.4$ & $626.0 \pm 41.4$ & $0.167 \pm 0.017$ \\
\hline & $5-6$ & $177.7 \pm 15.0$ & $634.8 \pm 41.3$ & $0.270 \pm 0.023$ \\
\hline & $6-8$ & $305.6 \pm 20.1$ & $1144.6 \pm 50.3$ & $0.301 \pm 0.016$ \\
\hline & $8-10$ & $219.6 \pm 16.3$ & $640.5 \pm 35.3$ & $0.329 \pm 0.020$ \\
\hline & $10-12$ & $109.4 \pm 11.0$ & $344.4 \pm 24.3$ & $0.316 \pm 0.024$ \\
\hline & $>12$ & $107.8 \pm 10.5$ & $332.7 \pm 23.8$ & $0.329 \pm 0.019$ \\
\hline
\end{tabular}

Table 4.7: The yields of the $D^{*+}$ and $D^{0}$ signal in each $p_{T}\left(D^{0}\right)$ region.

\begin{tabular}{cccc}
\hline \hline Dataset & $p_{T}\left(D^{0}\right)$ region & $D^{*+} / D^{0}$ ratio & $\epsilon\left(\pi_{s}\right)$ \\
\hline Muon & $<8 \mathrm{GeV} / c$ & $0.221 \pm 0.010$ & $0.673 \pm 0.037$ \\
& $>8 \mathrm{GeV} / c$ & $0.326 \pm 0.012$ & $0.993_{-0.049}^{+0.007}$ \\
\hline \multirow{2}{*}{ Electron } & $<8 \mathrm{GeV} / c$ & $0.232 \pm 0.013$ & $0.707 \pm 0.046$ \\
& $>8 \mathrm{GeV} / c$ & $0.330 \pm 0.021$ & $1.000_{-0.067}^{+0.000}$ \\
\hline \hline
\end{tabular}

Table 4.8: Summary of the slow pion reconstruction efficiency in each $D^{0}$ transverse momentum region. 


\subsubsection{Cross talk by $D^{* *}$}

Next we estimate the dilutions by $D^{* *}$ states. Following semileptonic $B^{-} / \bar{B}^{0}$ decay processes through $D^{* *}$ states cause cross talks of $B^{-} / \bar{B}^{0}$ in the $\ell^{-} D^{0}$ ( $D^{*+}$ excluded) and $\ell^{-} D^{*+}$ samples.

$$
\begin{aligned}
B^{-} & \rightarrow \ell^{-} \bar{\nu} D^{* * 0}, D^{* * 0} \rightarrow D^{*+} \pi^{+} \\
\bar{B}^{0} & \rightarrow \ell^{-} \bar{\nu} D^{* *+}, D^{* *+} \rightarrow D^{0} \pi^{-} \\
\bar{B}^{0} & \rightarrow \ell^{-} \bar{\nu} D^{* *+}, D^{* *+} \rightarrow D^{* 0} \pi^{-}, D^{* 0} \rightarrow D^{0} X^{0}
\end{aligned}
$$

To estimate the effect of the $D^{* *}$ cross talk, we use the $B^{-} / \bar{B}^{0}$ signal Monte Carlo sample with full detector simulation both for the muon and electron datasets. We split the full simulation sample according to the $D^{0}$ transverse momentum as done for the data in the previous section. Then we count the $B^{-} / \bar{B}^{0}$ contents each in the $\ell^{-} D^{0}\left(D^{*+}\right.$ excluded $)$ and $\ell^{-} D^{*+}$ sample. Tables 4.9-4.12 show summary of the compositions in the full simulation sample. In each table first one shows the composition of each simulated samples, and second one shows detailed composition of the $D^{* *}$ states in the same sample. The slow pion reconstruction efficiency is not considered in these tables. For the numbers from the second tables, we apply for the slow pion reconstruction efficiency measured in each region of the $D^{0}$ transverse momentum. Table 4.13 shows numbers from the full simulation sample after considering the slow pion reconstruction efficiency. From the table we define the $B^{-}$fraction $g_{-}$in each region and each sample $\left(\ell^{-} D^{0}\left(D^{*+}\right.\right.$ excluded $)$ and $\left.\ell^{-} D^{*+}\right)$ as,

$$
g_{-}=\frac{N_{B^{-}}}{N_{\bar{B}^{0}} \frac{\tau_{\bar{B}^{0}}}{\tau_{B^{-}}}+N_{B^{-}}},
$$

where $\tau_{\bar{B}^{0}}$ and $\tau_{B^{-}}$are the $\bar{B}^{0}$ and $B^{-}$meson lifetimes respectively. Since the number of $\ell^{-} D^{0}\left(D^{*+}\right)$ events is proportional to the lifetime and semileptonic partial width, the relative number of $\bar{B}^{0}$ with respect to $B^{-}$is corrected by their lifetime ratio. In Table $4.14, g_{-}$with $\tau\left(B^{-}\right) / \tau\left(\bar{B}^{0}\right)=1$ are summarized. 


\begin{tabular}{ccccc}
\hline \hline $\begin{array}{c}B \text { decay } \\
\text { type }\end{array}$ & \multicolumn{2}{c}{$\mu^{-} D^{0}$} & \multicolumn{2}{c}{$\mu^{-} D^{*+}$} \\
& $B^{0}$ & $B^{-}$ & $\bar{B}^{0}$ \\
\hline $\bar{B} \rightarrow \mu^{-} \bar{\nu} D$ & 423.8 & - & - & - \\
$\bar{B} \rightarrow \mu^{-} \bar{\nu} D^{*}$ & 2229.3 & - & - & 1475.2 \\
$\bar{B} \rightarrow \mu^{-} \bar{\nu} D^{* *}$ & 235.7 & 521.3 & 215.6 & 87.2 \\
\hline total & 2888.9 & 521.3 & 215.6 & 1562.4 \\
\hline \hline \multicolumn{6}{c}{} \\
\hline \hline$D^{* *}$ & $\mu^{-} D^{0}$ & $\mu^{-} D^{*+}$ \\
type & $B^{-}$ & $\bar{B}^{0}$ & $B^{-}$ & $\bar{B}^{0}$ \\
\hline${ }^{3} P_{0}$ & 7.4 & 20.9 & - & - \\
${ }^{3} P_{1}$ & 2.9 & 14.5 & 5.2 & 3.4 \\
${ }^{1} P_{1}$ & 73.2 & 159.3 & 106.6 & 42.6 \\
${ }^{3} P_{2}$ & 105.2 & 217.4 & 50.5 & 19.8 \\
NR & 47.1 & 109.2 & 53.3 & 21.4 \\
\hline total & 235.7 & 521.3 & 215.6 & 87.2 \\
\hline \hline
\end{tabular}

Table 4.9: Composition in full simulation samples for the muon dataset, $p_{T}\left(D^{0}\right)<8 \mathrm{GeV} / c$. NR means Non-Resonant decay, which is also counted as a $D^{* *}$ state. In the lower table, ${ }^{3} P_{0}$ states decay only to $D \pi,{ }^{3} P_{1},{ }^{1} P_{1}$ decay only to $D^{*} \pi .{ }^{3} P_{2}$ and Non-Resonant states decay to both $D \pi$ and $D^{*} \pi$.

\begin{tabular}{ccccc}
\hline \hline $\begin{array}{c}B \text { decay } \\
\text { type }\end{array}$ & \multicolumn{2}{c}{$\mu^{-} D^{0}$} & \multicolumn{2}{c}{$\mu^{-} D^{*+}$} \\
$\bar{B} \rightarrow \mu^{-} \bar{\nu} D$ & 453.3 & - & - & \\
$\bar{B} \rightarrow \mu^{-} \bar{\nu} D^{*}$ & 1425.4 & - & - & 944. \\
$\bar{B} \rightarrow \mu^{-} \bar{\nu} D^{* *}$ & 170.4 & 395.4 & 149.4 & 74.2 \\
\hline total & 2049.1 & 395.4 & 149.4 & 10 \\
\hline \hline \multicolumn{6}{c}{} \\
\hline \hline$D^{* *}$ & $\mu^{-} D^{0}$ & \multicolumn{4}{c}{$\mu^{-} D^{*+}$} \\
type & $B^{-}$ & $\bar{B}^{0}$ & $B^{-}$ & $\bar{B}^{0}$ \\
\hline${ }^{3} P_{0}$ & 5.3 & 16.6 & - & - \\
${ }^{3} P_{1}$ & 3.3 & 12.1 & 5.3 & 3.1 \\
${ }^{1} P_{1}$ & 50.5 & 106.2 & 73.2 & 37.6 \\
${ }^{3} P_{2}$ & 68.2 & 143.1 & 26.0 & 13.7 \\
NR & 43.0 & 117.4 & 44.9 & 19.9 \\
\hline total & 170.4 & 395.4 & 149.4 & 74.2 \\
\hline \hline
\end{tabular}

Table 4.10: Same with the previous table, but for $p_{T}\left(D^{0}\right)>8 \mathrm{GeV} / c$. 


\begin{tabular}{ccccc}
\hline \hline$B$ decay & \multicolumn{2}{c}{$e^{-} D^{0}$} & \multicolumn{2}{c}{$e^{-} D^{*+}$} \\
type & $B^{-}$ & $\bar{B}^{0}$ & $B^{-}$ & $\bar{B}^{0}$ \\
\hline $\bar{B} \rightarrow e^{-} \bar{\nu} D$ & 336.6 & - & - & - \\
$\bar{B} \rightarrow e^{-} \bar{\nu} D^{*}$ & 1634.1 & - & - & 1100.2 \\
$\bar{B} \rightarrow e^{-} \bar{\nu} D^{* *}$ & 164.4 & 359.7 & 142.0 & 65.0 \\
\hline total & 2135.2 & 359.7 & 142.0 & 1165.2 \\
\hline \hline
\end{tabular}

\begin{tabular}{ccccc}
\hline \hline$D^{* *}$ & \multicolumn{2}{c}{$e^{-} D^{0}$} & \multicolumn{2}{c}{$e^{-} D^{*+}$} \\
type & $B^{-}$ & $\bar{B}^{0}$ & $B^{-}$ & $\bar{B}^{0}$ \\
\hline${ }^{3} P_{0}$ & 5.6 & 9.2 & - & - \\
${ }^{3} P_{1}$ & 2.1 & 6.0 & 3.4 & 0.7 \\
${ }^{1} P_{1}$ & 51.8 & 119.5 & 77.3 & 35.6 \\
${ }^{3} P_{2}$ & 76.1 & 162.1 & 30.8 & 16.7 \\
NR & 28.9 & 63.0 & 30.5 & 12.0 \\
\hline total & 164.4 & 359.7 & 142.0 & 65.0 \\
\hline \hline
\end{tabular}

Table 4.11: Same but for electron dataset, $p_{T}\left(D^{0}\right)<8 \mathrm{GeV} / c$.

\begin{tabular}{ccccc}
\hline \hline $\begin{array}{c}B \text { decay } \\
\text { type }\end{array}$ & \multicolumn{2}{c}{$e^{-} D^{0}$} & \multicolumn{2}{c}{$e^{-} D^{*+}$} \\
$\bar{B} \rightarrow e^{-} \bar{\nu} D$ & 247.5 & - & - & - \\
$\bar{B} \rightarrow e^{-} \bar{\nu} D^{*}$ & 723.4 & - & - & 484.8 \\
$\bar{B} \rightarrow e^{-} \bar{\nu} D^{* *}$ & 73.5 & 156.4 & 61.6 & 30.1 \\
\hline total & 1044.4 & 156.4 & 61.6 & 515.0 \\
\hline \hline \multicolumn{6}{c}{} \\
\hline \hline$D^{* *}$ & \multicolumn{6}{c}{$e^{-} D^{0}$} & \multicolumn{2}{c}{$e^{-} D^{*+}$} \\
type & $B^{-}$ & $\bar{B}^{0}$ & $B^{-}$ & $\bar{B}^{0}$ \\
\hline${ }^{3} P_{0}$ & 3.1 & 4.0 & - & - \\
${ }^{3} P_{1}$ & 1.8 & 2.5 & 2.0 & 0.5 \\
${ }^{1} P_{1}$ & 24.8 & 50.0 & 31.6 & 16.7 \\
${ }^{3} P_{2}$ & 28.4 & 63.6 & 10.8 & 5.6 \\
NR & 15.6 & 36.4 & 17.2 & 7.3 \\
\hline total & 73.5 & 156.4 & 61.6 & 30.1 \\
\hline \hline
\end{tabular}

Table 4.12: Same for electron dataset, but $p_{T}\left(D^{0}\right)>8 \mathrm{GeV} / c$. 


\begin{tabular}{cccccc}
\hline \hline \multirow{2}{*}{ Dataset } & $p_{T}\left(D^{0}\right)$ & \multicolumn{2}{c}{$\ell^{-} D^{0}$} & \multicolumn{2}{c}{$\ell^{-} D^{*+}$} \\
range & $N_{B^{-}}$ & $N_{\bar{B}^{0}}$ & $N_{B^{-}}$ & $N_{\bar{B}^{0}}$ \\
\hline Muon & $<8 \mathrm{GeV} / c$ & 2959.4 & 1032.2 & 145.1 & 1051.5 \\
& $>8 \mathrm{GeV} / c$ & 2050.1 & 402.5 & 148.4 & 1011.4 \\
\hline \multirow{2}{*}{ Electron } & $<8 \mathrm{GeV} / c$ & 2176.8 & 701.1 & 100.4 & 823.8 \\
& $>8 \mathrm{GeV} / c$ & 1044.4 & 156.4 & 61.6 & 515.0 \\
\hline \hline
\end{tabular}

Table 4.13: Composition in full simulation samples after considering the slow pion reconstruction efficiency.

\begin{tabular}{cccc}
\hline \hline Dataset & $p_{T}\left(D^{0}\right)$ range & $\ell^{-} D^{0}$ & $\ell^{-} D^{*+}$ \\
\hline Muon & $<8 \mathrm{GeV} / c$ & 0.741 & 0.121 \\
& $>8 \mathrm{GeV} / c$ & 0.836 & 0.128 \\
\hline Electron & $<8 \mathrm{GeV} / c$ & 0.756 & 0.109 \\
& $>8 \mathrm{GeV} / c$ & 0.870 & 0.107 \\
\hline \hline
\end{tabular}

Table 4.14: Table of $g_{-}$( $B^{-}$fraction) in each sample with $\tau\left(B^{-}\right) / \tau\left(\bar{B}^{0}\right)=1$. 

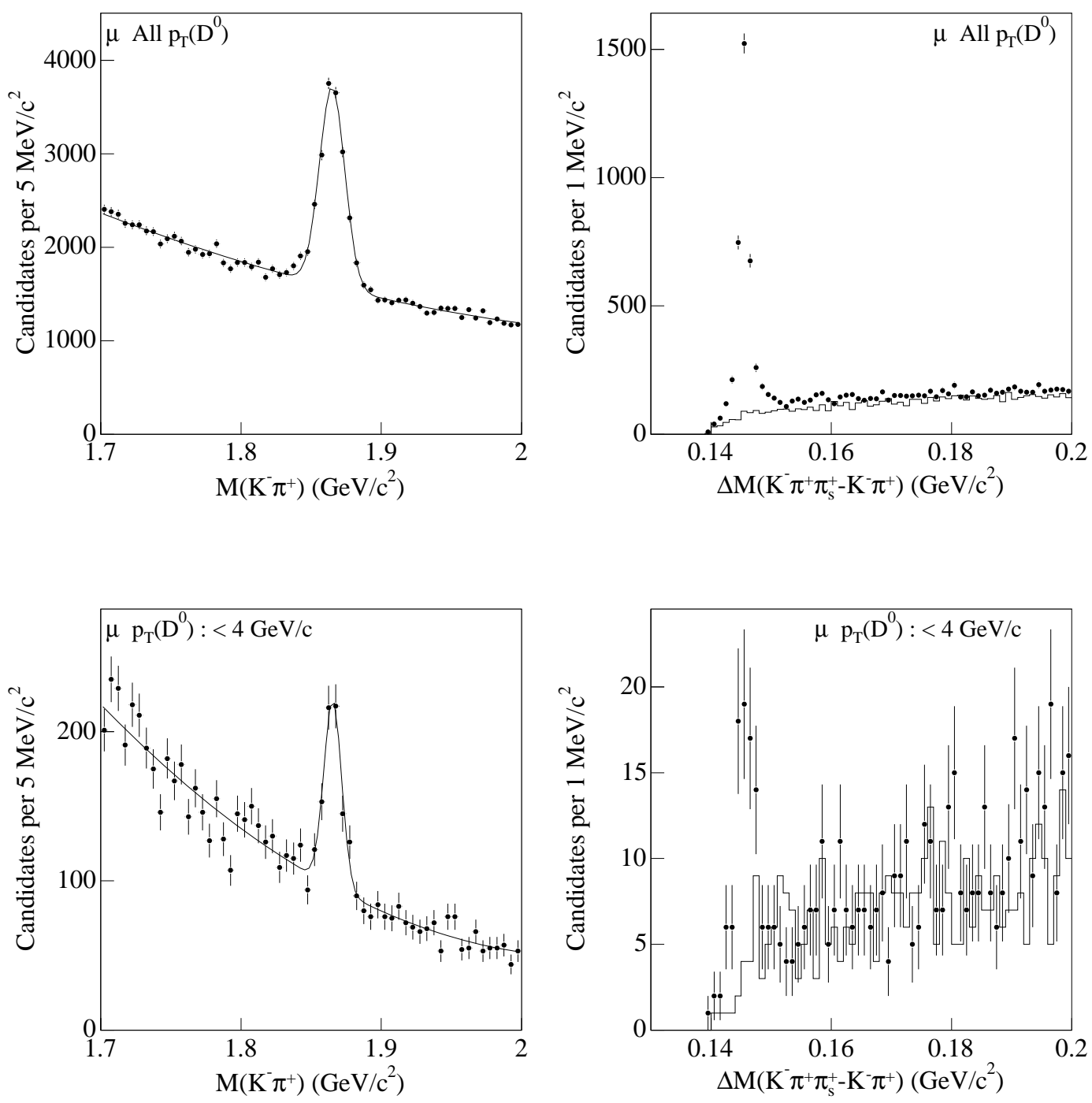

Figure 4.11: Distributions of $M\left(K^{-} \pi^{+}\right)$and $\Delta M\left(K^{-} \pi^{+} \pi_{s}^{+}-K^{-} \pi^{+}\right)$in different $D^{0}$ transverse momentum range. These are for the muon dataset. 

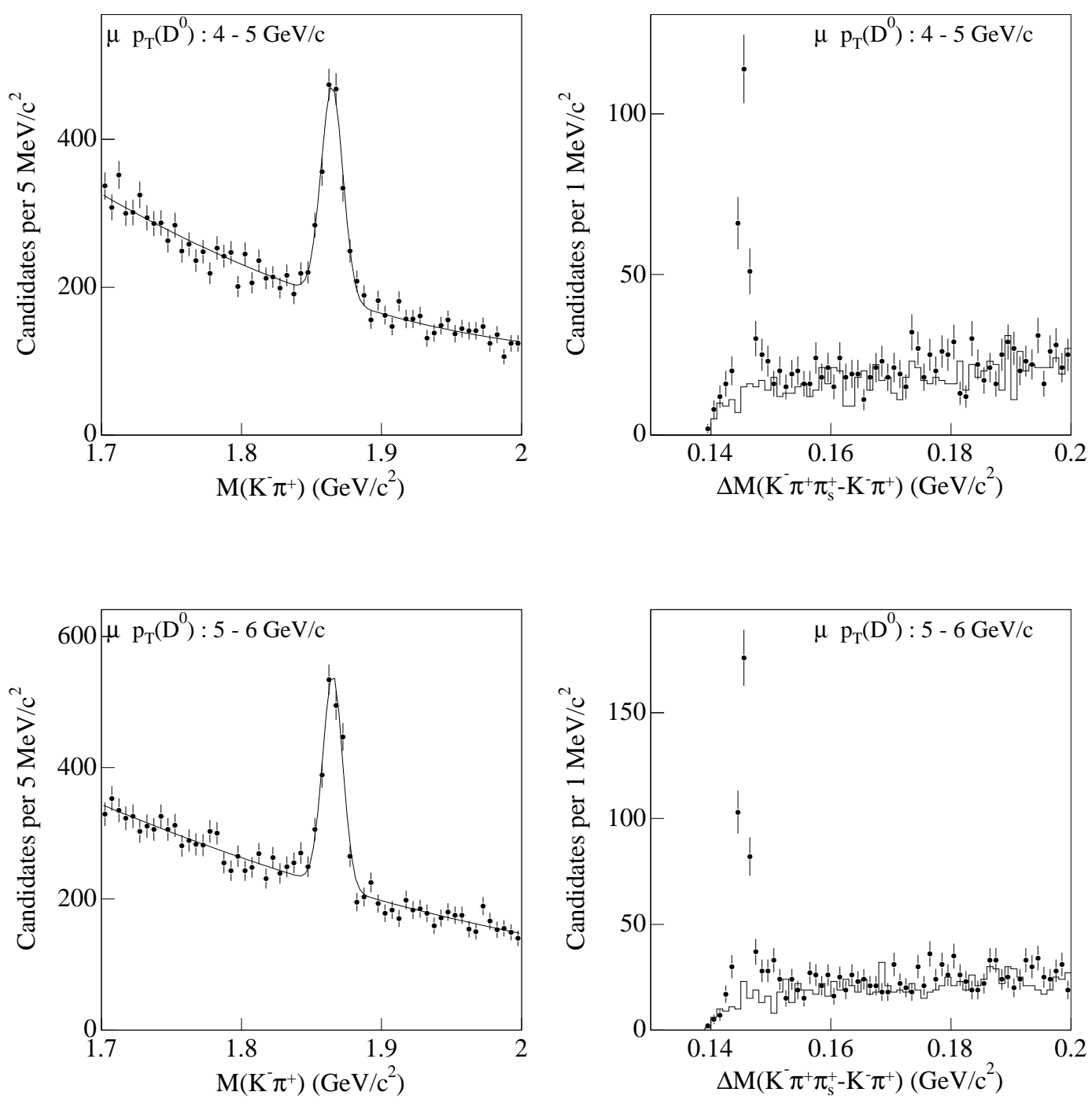

Figure 4.12: Same as previous figures but higher momentum range (4-5, 5$6 \mathrm{GeV} / c)$. 

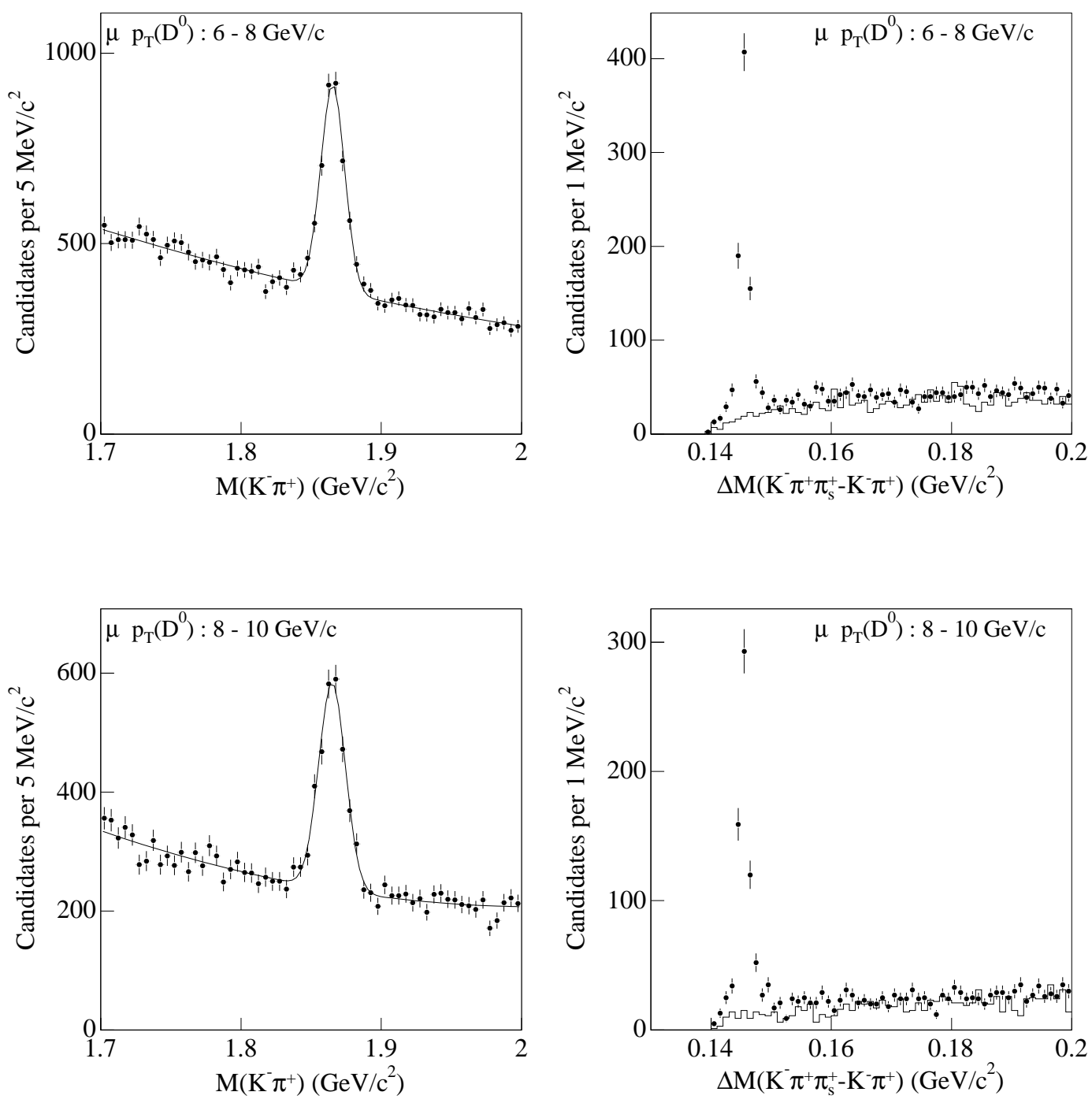

Figure 4.13: Same as previous figures but higher momentum range $(6-8,8-$ $10 \mathrm{GeV} / c$ ). 

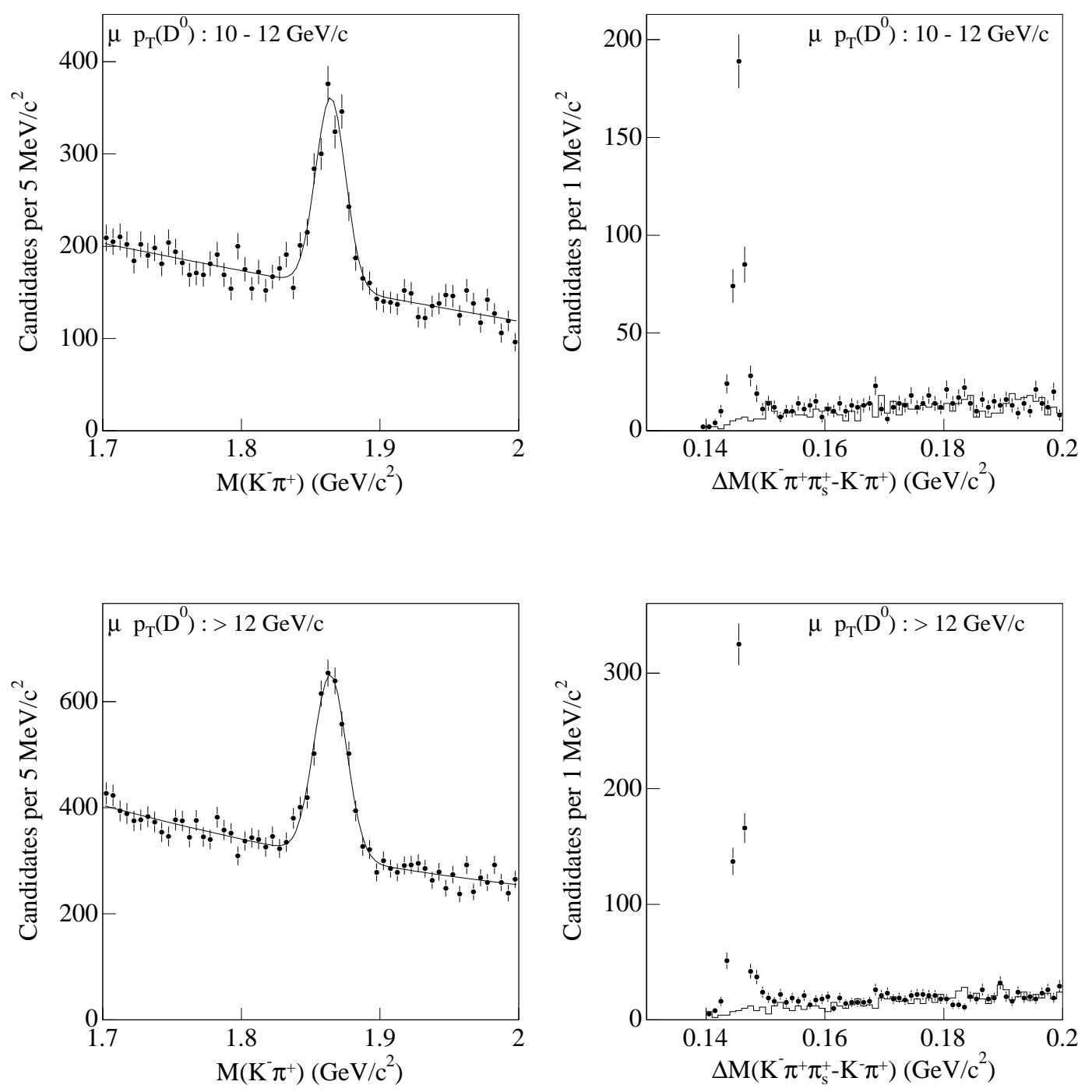

Figure 4.14: Same as previous figures but higher momentum range (10-12, $>12 \mathrm{GeV} / c$ ). 

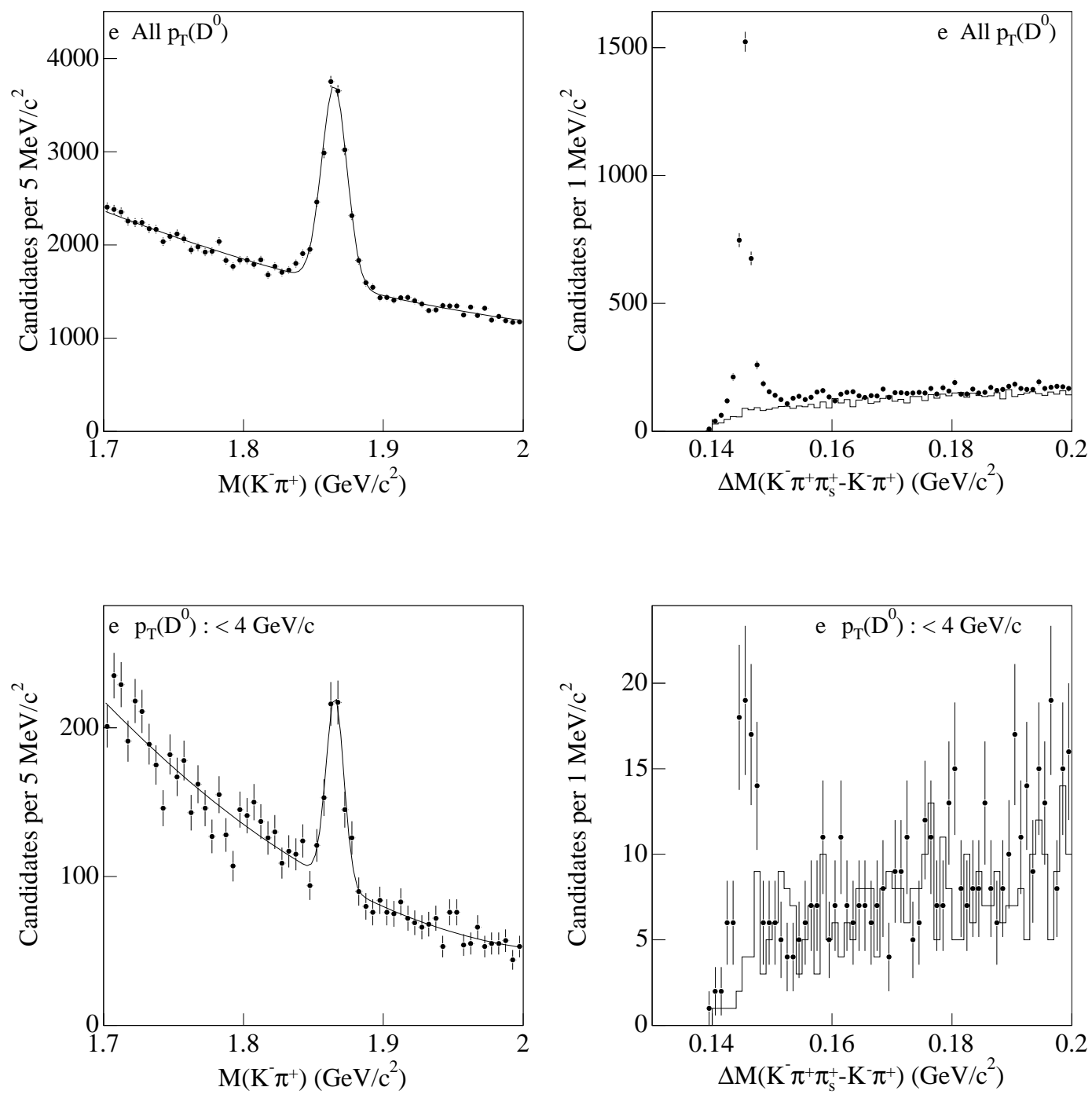

Figure 4.15: Distributions of $M\left(K^{-} \pi^{+}\right)$and $\Delta M\left(K^{-} \pi^{+} \pi_{s}^{+}-K^{-} \pi^{+}\right)$in different $D^{0}$ transverse momentum range. These are for the electron dataset. 

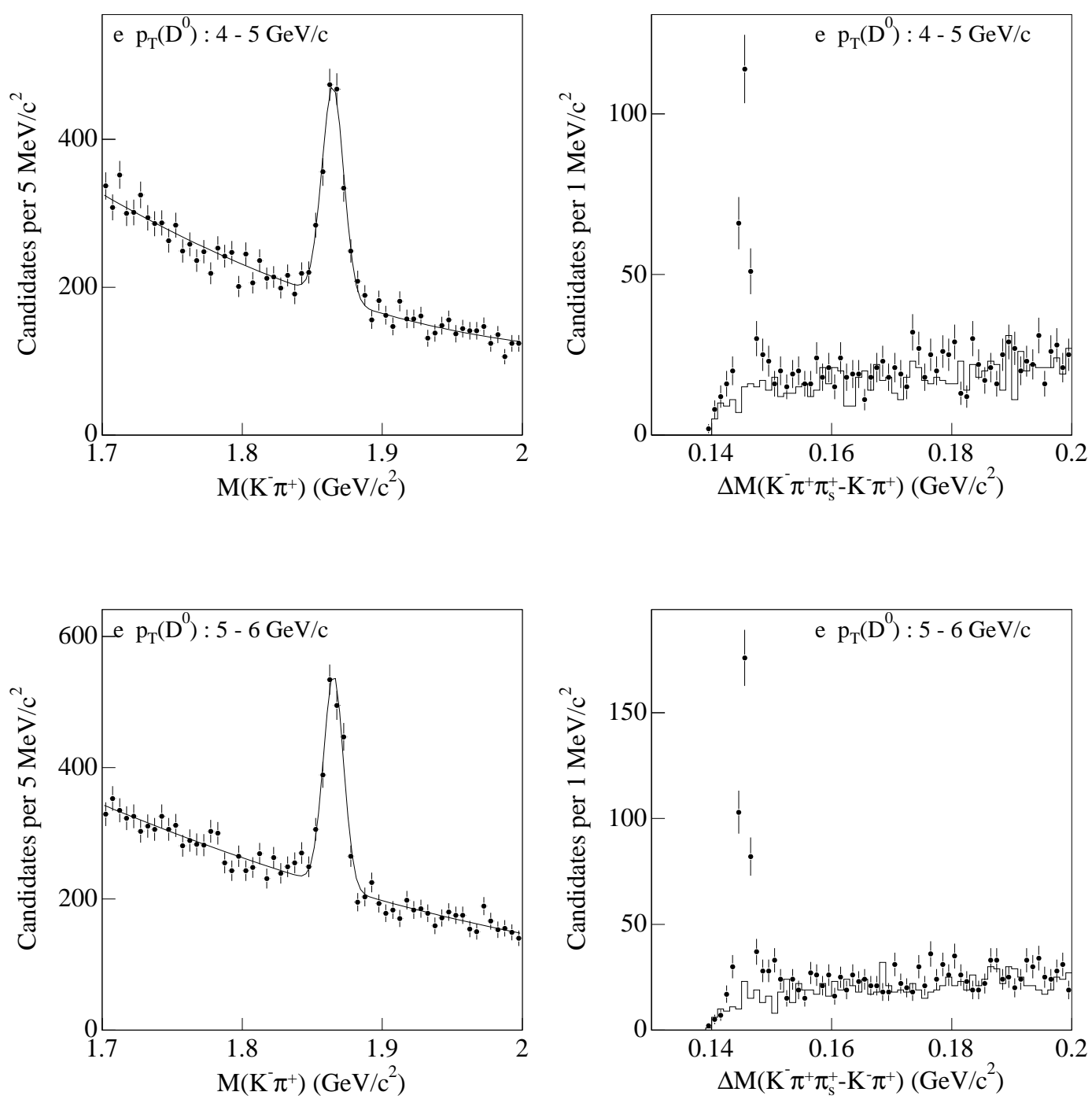

Figure 4.16: Same as previous figures but higher momentum range (4-5, 5$6 \mathrm{GeV} / c)$. 

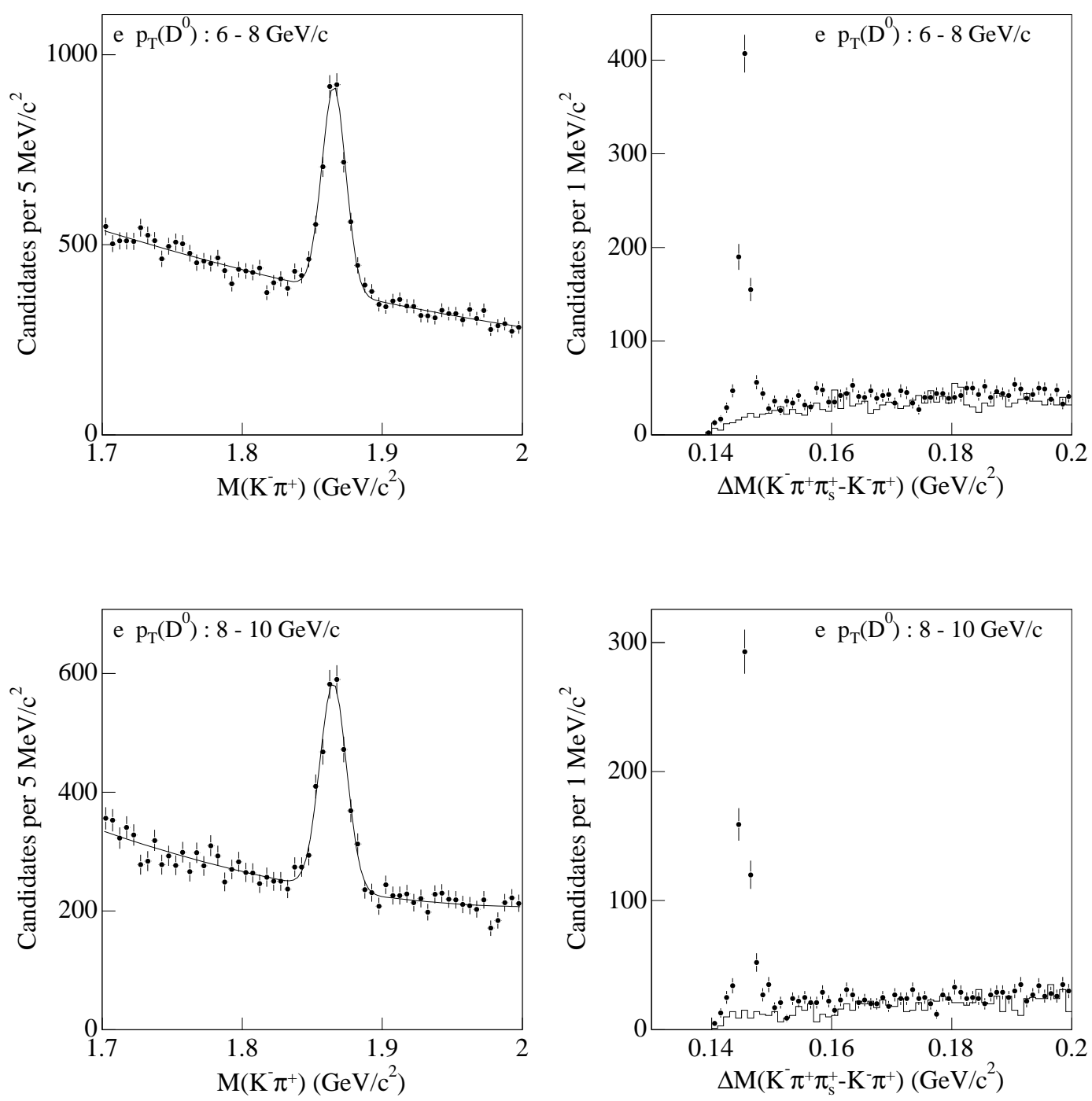

Figure 4.17: Same as previous figures but higher momentum range $(6-8,8-$ $10 \mathrm{GeV} / c$ ). 

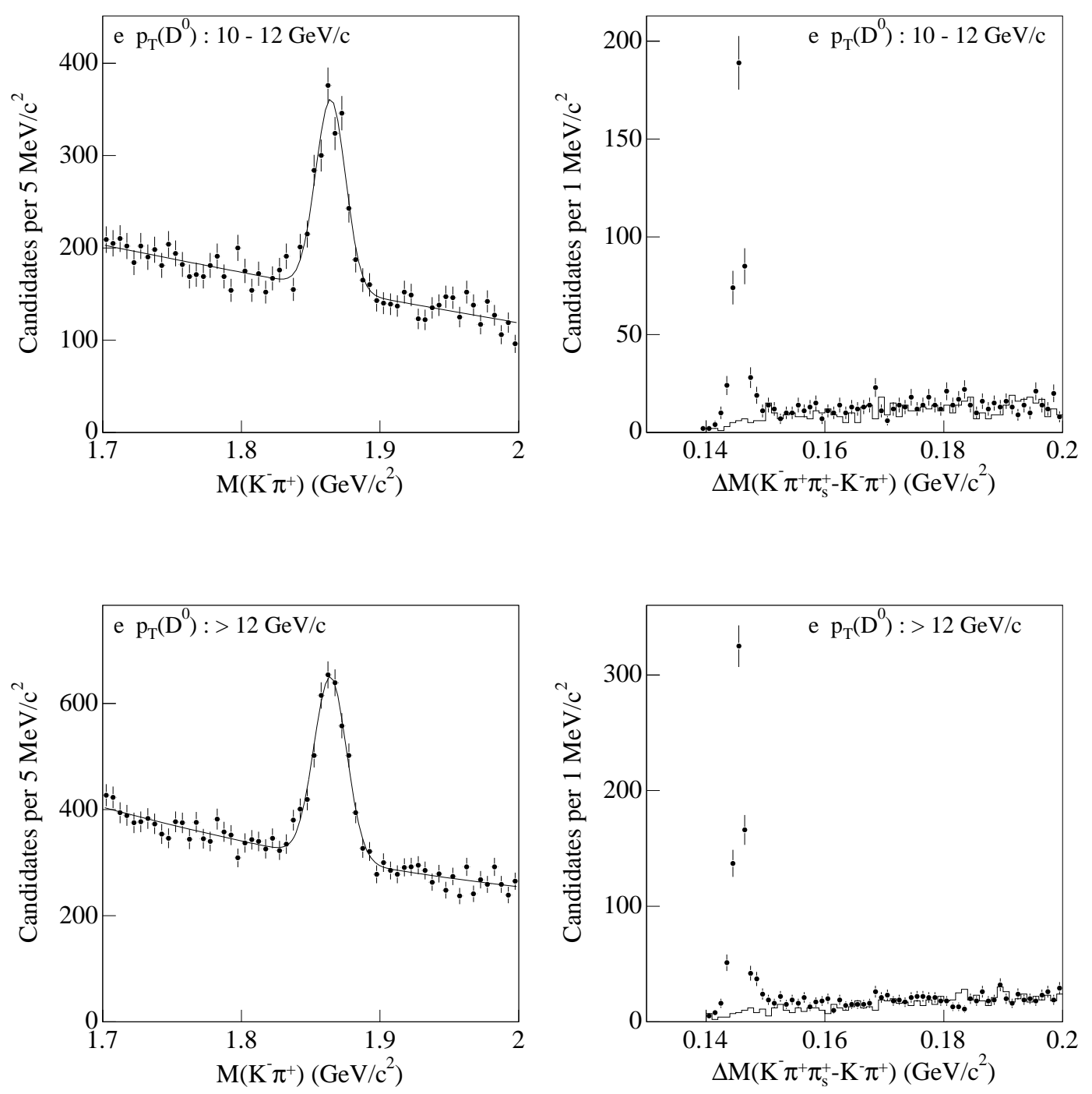

Figure 4.18: Same as previous figures but higher momentum range (10-12, > $12 \mathrm{GeV} / c$ ). 

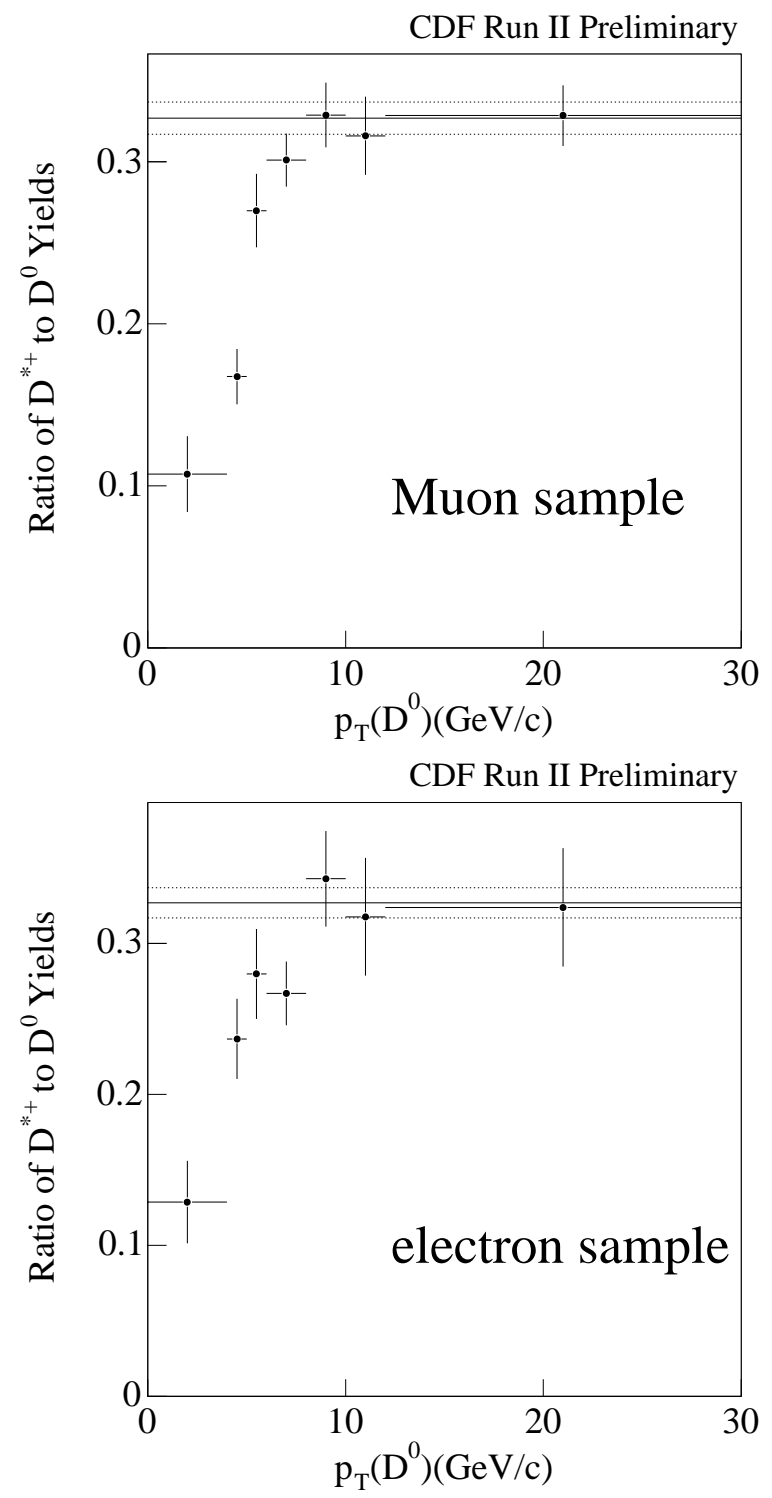

Figure 4.19: The ratio of $D^{*+} / D^{0}$ yield against $D^{0}$ meson transverse momentum. The lines show a level of plateau, which corresponds to the tracking efficiency of $99.6 \%$. 

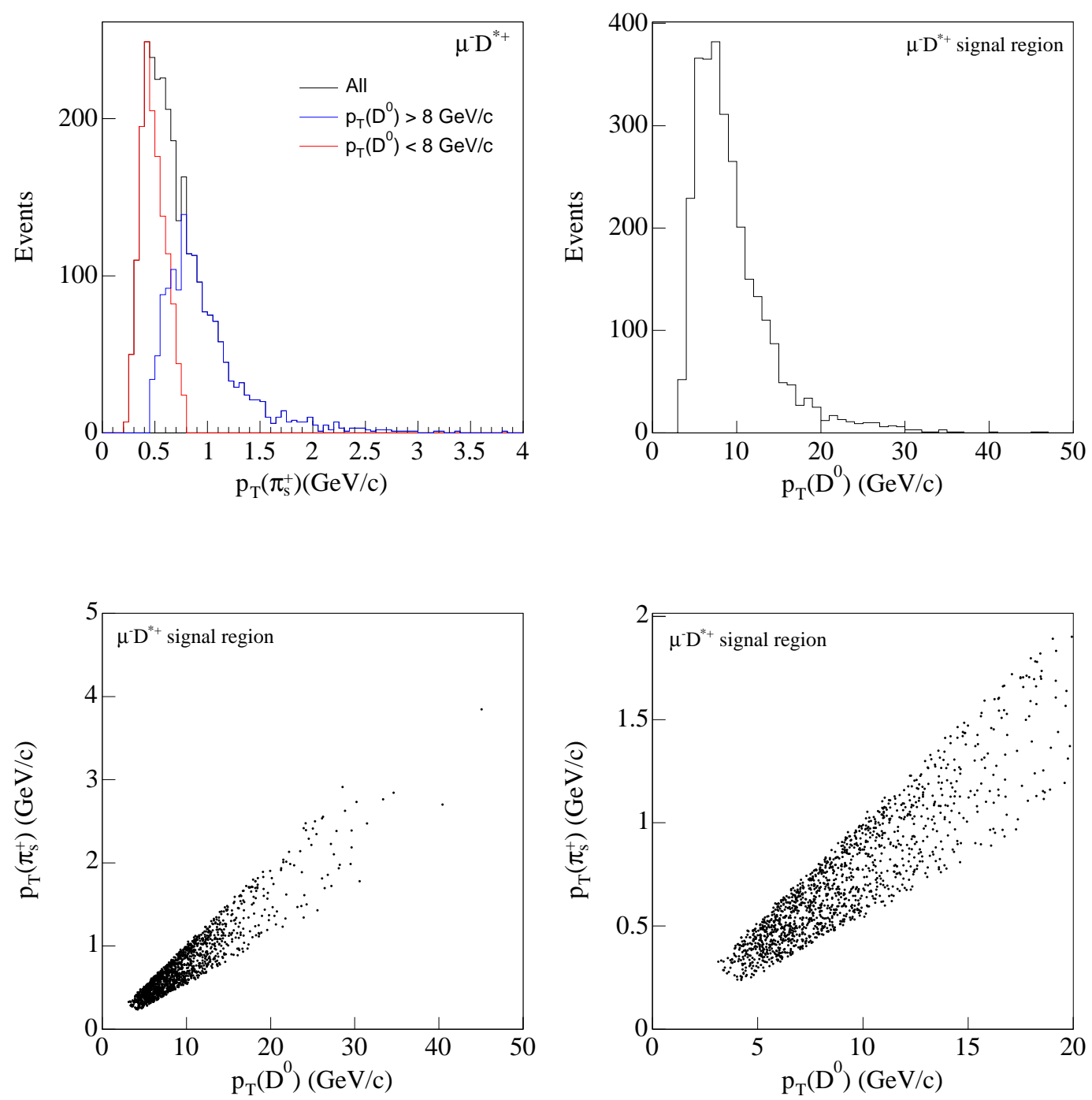

Figure 4.20: The $p_{T}$ distributions of the slow pion and the $D^{0}$ meson, and their correlation. The plots are for $\mu^{-} D^{*+}$ signal region events. Lower two correlation plots are same but with different scale. 


\subsection{Lifetime fit}

We define the probability density function for the semileptonic $B^{-} / \bar{B}^{0}$ decays as follows.

$$
\mathcal{F}_{B}\left(c t^{*}, \sigma_{c t^{*}} ; c \tau, s\right)=\operatorname{Exp}\left(-c t^{*} ; c \tau / K\right) \otimes H(K) \otimes G\left(s \sigma_{c t^{*}}\right)
$$

where $H(K)$ denotes the $K$ factor distribution, $G(\sigma)$ means Gaussian smearing with the resolution $\sigma$. An exact formulation of $\mathcal{F}_{B}$ is shown in Appendix A.1.

From this formula we define probability density functions for the $B^{-}$and $\bar{B}^{0}$ mesons each in the $\mu^{-} D^{0}, \mu^{-} D^{*+}, e^{-} D^{0}$ and $e^{-} D^{*+}$ sample. These functions defined in each sample have the different $K$ factor, but have the uniform $c \tau$ each for the $B^{-}$and $\bar{B}^{0}$ meson.

Then we define the event probability density function as follows. In the formula $i$ means an event number in each sample.

$$
\begin{array}{ccc}
P_{i}\left(c t^{*}(i), \sigma_{c t^{*}}(i)\right)= & f_{\text {sig }}\left\{\left(1-f_{c}-f_{b}\right) \mathcal{F}_{\mathrm{sl}}+f_{c} \mathcal{F}_{c}+f_{b} \mathcal{F}_{b}\right\}+\left(1-f_{\text {sig }}\right) \mathcal{F}_{\mathrm{BG}} \\
& \mathcal{F}_{\mathrm{sl}}=g_{-} \mathcal{F}_{B^{-}}+\left(1-g_{-}\right) \mathcal{F}_{\bar{B}^{0}} \\
f_{\text {sig }} & \ldots & D^{0}\left(\text { or } D^{*+}\right) \text { fraction in signal region obtained from the } \\
& M\left(K^{-} \pi^{+}\right) \text {distribution. } \\
\mathcal{F}_{\mathrm{BG}} & \ldots & \text { Combinatorial background function. } \\
f_{c}, \mathcal{F}_{c} & \ldots & \text { Fraction and shape of the charm background. } \\
f_{b}, \mathcal{F}_{b} & \ldots & \text { Fraction and shape of the bottom background. } \\
g_{-} & \ldots & \text { Fraction of } B^{-} \text {component. }
\end{array}
$$

For the value of the probability density function for $i$-th event, we obtain the event likelihood $l_{i}$, and form the combined likelihood as,

$$
L_{X}=\prod_{i} l_{i}\left(\mathrm{X}=\mu^{-} D^{0}, \mu^{-} D^{*+}, e^{-} D^{0}, e^{-} D^{*+}\right)
$$

In advance to the combined fit, we fit the muon and electron samples separately by maximizing following likelihoods respectively.

$$
\begin{aligned}
L_{\mu} & =L_{\mu D^{0}} \cdot L_{\mu D^{*+}} \\
L_{e} & =L_{e D^{0}} \cdot L_{e D^{*+}}
\end{aligned}
$$

We maximize these likelihoods by adjusting values of $c \tau\left(B^{-}\right)$and $c \tau\left(\bar{B}^{0}\right)$. Thus we obtain the following lifetimes and ratio.

For the muon sample:

$$
\begin{aligned}
c \tau\left(B^{-}\right) & =489.8 \pm 10.8 \mu \mathrm{m} \\
c \tau\left(\bar{B}^{0}\right) & =453.6 \pm 13.6 \mu \mathrm{m} \\
\tau\left(B^{-}\right) / \tau\left(\bar{B}^{0}\right) & =1.080 \pm 0.048
\end{aligned}
$$


For the electron sample:

$$
\begin{aligned}
c \tau\left(B^{-}\right) & =504.7 \pm 13.9 \mu \mathrm{m} \\
c \tau\left(\bar{B}^{0}\right) & =418.1 \pm 18.2 \mu \mathrm{m} \\
\tau\left(B^{-}\right) / \tau\left(\bar{B}^{0}\right) & =1.207 \pm 0.072
\end{aligned}
$$

The fit results for the muon and electron datasets are shown in Figure 4.21.

Then we form the combined likelihood from $L_{\mu}$ and $L_{e}$.

$$
L_{\text {combined }}=L_{\mu} \cdot L_{e}
$$

As a result of the combined fit we obtained

$$
\begin{aligned}
c \tau\left(B^{-}\right) & =495.6 \pm 8.6 \mu \mathrm{m} \\
c \tau\left(\bar{B}^{0}\right) & =441.5 \pm 10.9 \mu \mathrm{m} \\
\tau\left(B^{-}\right) / \tau\left(\bar{B}^{0}\right) & =1.123 \pm 0.040
\end{aligned}
$$

\begin{tabular}{|c|c|c|}
\hline Dataset & $\ell^{-} D^{0}\left(D^{*+}\right.$ excluded $)$ & $\ell^{-} D^{*+}$ \\
\hline$\mu$ only & 0.8562 & 0.1071 \\
\hline$e$ only & 0.0484 & 0.8091 \\
\hline Combined & 0.7538 & 0.0999 \\
\hline
\end{tabular}

The uncertainties are statistical only. The correlation coefficient in the $\mathrm{c} \tau\left(B^{-}\right)$ and $\mathrm{c} \tau\left(\bar{B}^{0}\right)$ uncertainties is -0.418 , and is included in the uncertainty of $\tau\left(B^{-}\right) / \tau\left(\bar{B}^{0}\right)$. The $c t^{*}$ distributions and fit results are shown in Figure 4.22. Fit probabilities in each fit are summarized in Table 4.15. The table of $g_{-}$ determined by the fit is found in Table 4.16.

Table 4.15: Fitting probabilities calculated from each fit result.

\begin{tabular}{cccc}
\hline \hline Dataset & $p_{T}\left(D^{0}\right)$ range & $\ell^{-} D^{0}$ & $\ell^{-} D^{*+}$ \\
\hline Muon & $<8 \mathrm{GeV} / c$ & 0.763 & 0.134 \\
& $>8 \mathrm{GeV} / c$ & 0.851 & 0.141 \\
\hline Electron & $<8 \mathrm{GeV} / c$ & 0.777 & 0.120 \\
& $>8 \mathrm{GeV} / c$ & 0.882 & 0.118 \\
\hline \hline
\end{tabular}

Table 4.16: The $g_{-}$( $B^{-}$fraction $)$in each sample determined by the combined fit. 

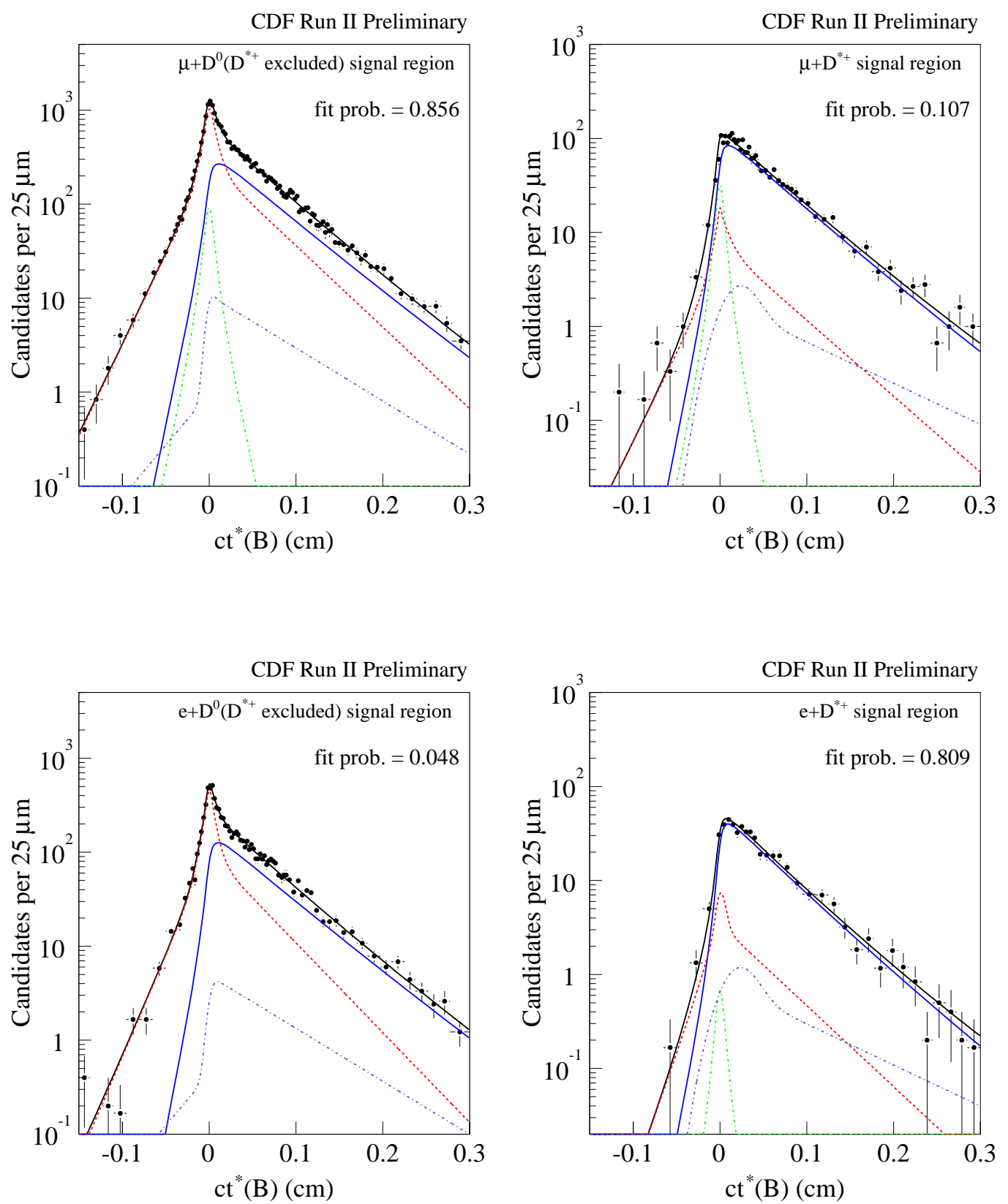

Figure 4.21: The $c t^{*}$ distributions (points) and the fit results for the muon dataset (upper) and for the electron (lower). Blue curves show the semileptonic $B^{-} / \bar{B}^{0}$ signal components, Red curves are the combinatorial background shape. Dashed green and blue curves show charm and bottom background components, respectively. 


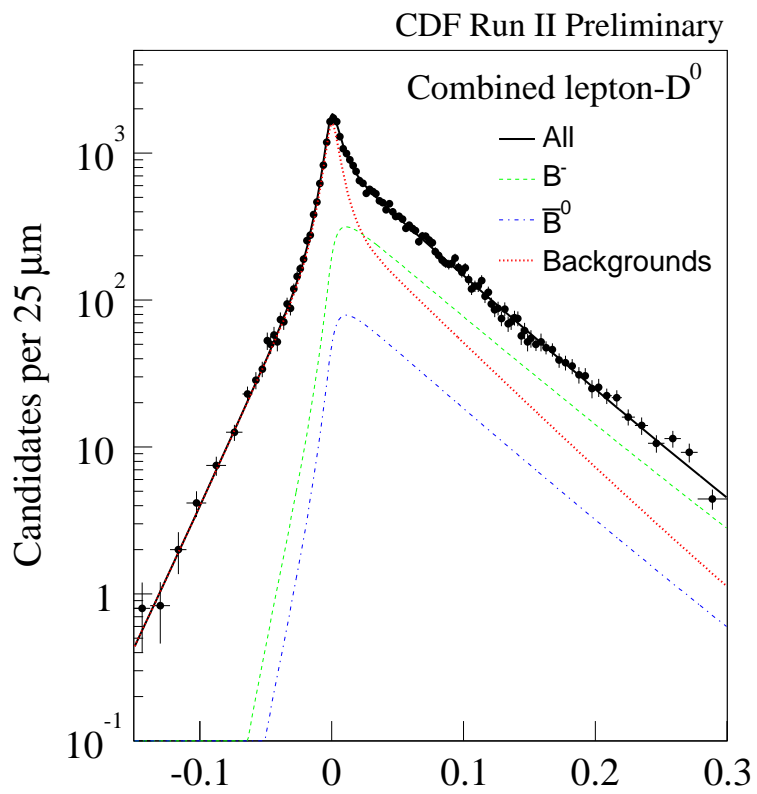

Pseudo-proper Decay Length $(\mathrm{cm})$

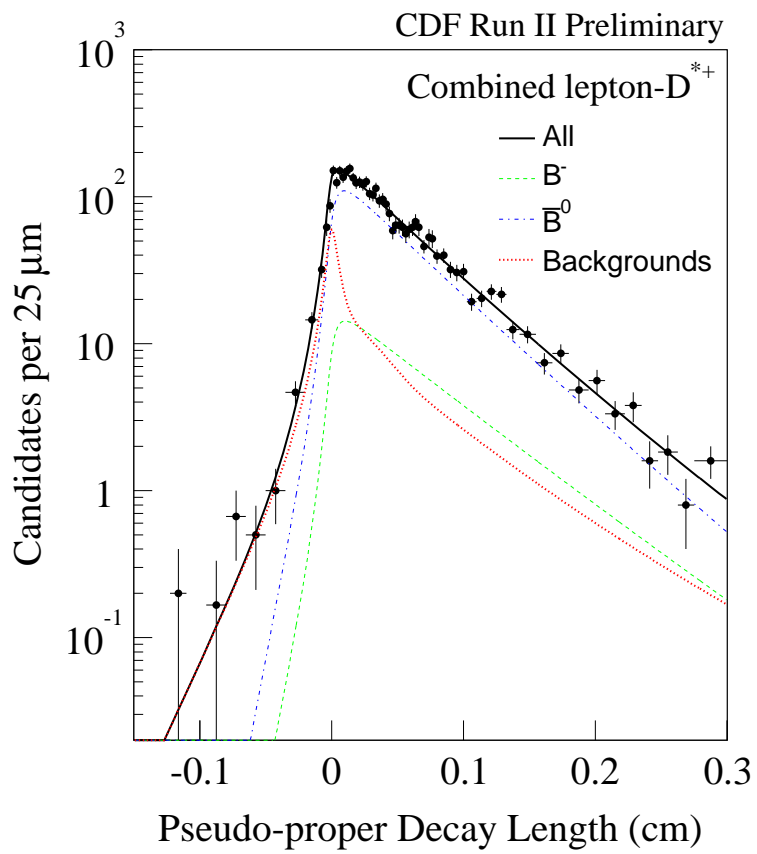

Figure 4.22: The muon and electron combined fit result. 


\subsection{Systematic uncertainties}

Now we evaluate the systematic uncertainties of the $B^{-} / \bar{B}^{0}$ lifetime measurement from several possible sources.

\subsubsection{Physics backgrounds}

First major sources of the systematics are the physics background treatments. We estimate these effects as follows. For the charm background fraction, we look the effect to the lifetimes by changing $f_{c}$ about one standard deviation from the measured value. For the charm background shape $\mathcal{F}_{c}$, we change the shape from $\mathcal{F}_{c}^{\text {zero }}$ to $\mathcal{F}_{c}^{\text {non-zero }}$ as described in Section 4.5.2. For the bottom background, we use the basically same procedure with the charm background case. We change the values of $f_{b}$ according to its uncertainties. As explained in Section 4.5.3, to consider the contribution from the residual $h^{-} D^{0}$ in bottom background, we increase $f_{b}$ about $+0.5 \%$ in addition to its statistical uncertainties. Also, we change $\mathcal{F}_{b}$ from of non-semileptonic $\ell^{-} D^{0}$ to of $h^{-} D^{0}$ events. We assign the observed changes of the lifetimes as the systematic uncertainties from the physics backgrounds.

\subsubsection{Sample composition}

The incompleteness of the sample composition estimation is another major source of the systematics. In semileptonic $B^{-} / \bar{B}^{0}$ meson lifetime analysis performed in CDF Run I [7] it gives the largest contribution to the systematic uncertainties. The similar situation is expected in Run II.

Relating to the sample composition estimation, there are three important parameters. The first parameter is $f^{* *}$ which is a decay fraction to $\ell^{-} \bar{\nu} D^{* *}$ in the semileptonic $B^{-} / \bar{B}^{0}$ decays.

$$
f^{* *}=\frac{\mathcal{B}\left(\bar{B} \rightarrow \ell^{-} \bar{\nu} D^{* *}\right)}{\mathcal{B}\left(\bar{B} \rightarrow \ell^{-} \bar{\nu} D X\right)}
$$

where denominator means total branching fraction of all the $\bar{B}$ semileptonic decays.

The second one is a branching fraction of $D^{* *} \rightarrow D^{*} \pi$ transition, called $P_{V}$.

$$
P_{V}=\frac{\mathcal{B}\left(D^{* *} \rightarrow D^{*} \pi\right)}{\mathcal{B}\left(D^{* *} \rightarrow D^{*} \pi\right)+\mathcal{B}\left(D^{* *} \rightarrow D \pi\right)}
$$

As stated in Section 4.6.2, since the dilutions mainly come through the $D^{* *}$ states, inaccurate $f^{* *}$ and $P_{V}$ values cause relatively large systematic uncertainties. 
The last parameter is the slow pion reconstruction efficiency $\epsilon\left(\pi_{s}\right)$ measured in Section 4.6.1. We change these three parameters and repeat the lifetime fits.

The default values of $f^{* *}$ and $P_{V}$ used in the $B^{-} / \bar{B}^{0}$ Monte Carlo samples are $f^{* *}=0.36$ and $P_{V}=0.651$. We change $f^{* *}$ in the range $0.24-0.48$. Changing $P_{V}$ is also examined in the range 0.22 - 1.0. Changing these numbers changes the contents of Table 4.9-4.12, giving a new set of $g_{-}$and the $K$ factor distributions. According to the new values of $f^{* *}$ and $P_{V}$, we re-weight the events in the $B^{-} / \bar{B}^{0}$ Monte Carlo samples, then calculate $g_{-}$and corresponding $K$ factor distributions. The lifetime fits are repeated with these new configurations. Plots in Figure 4.23 and 4.24 show the changes of $g_{-}$as function of $f^{* *}$ and $P_{V}$, in each $\ell^{-} D^{0}\left(\ell^{-} D^{*+}\right)$ sample, each $D^{0}$ transverse momentum region. The changes of $g_{-}$and lifetimes according to $f^{* *}$ and $P_{V}$ changes are also summarized in Tables 4.17 and 4.18. We also test the change of the slow pion reconstruction efficiency. We examine the values of $\epsilon\left(\pi_{s}\right) \pm$ one standard deviation from the measured values. The change of $g_{-}$and lifetimes are also summarized in Tables 4.17, 4.18. We assign the changes shown in the Table 4.18 as the systematic uncertainties originated from the sample composition uncertainty.

\subsubsection{Missing momentum correction}

The missing momentum correction, namely the $K$ factor distribution becomes another source of the systematics. We consider three conditions contributing to the $K$ factor distribution, namely $p_{T}(B)$ spectrum, $B$ decay model, and electron cuts.

The first one comes from inaccuracy of the $B^{-} / \bar{B}^{0}$ meson transverse momentum spectrum used in the $B^{-} / \bar{B}^{0}$ Monte Carlo. As discussed in Section 4.1 , we tune the $p_{T}(B)$ spectrum with a correction factor $p_{T}(B)^{\alpha}$, where the measured $\alpha$ is -0.7320 . We examine the value of $\alpha$ from 0 to -1.4640 , where the absolute shift width is two times larger than the measured value.

The second one is dependence of the $B$ decay model used in the $B^{-} / \bar{B}^{0}$ signal Monte Carlo simulation. By default ISGW model [62] is employed for the semileptonic $B^{-} / \bar{B}^{0}$ decays. We change it to the simple $\mathrm{V}-\mathrm{A}$ decays, and fit the lifetimes using a new set of the $K$ factors.

The last one related to the $K$ factor is effect of the electron ID cuts. As explained in Section 4.2, we use a full simulation sample to get the electron $K$ factors. We change the electron $K$ factor distributions to the ones from the parametric Monte Carlo sample, which are identical with the muon $K$ factor distributions.

The changes of the lifetime fit arising from each configuration change are assigned as the systematic uncertainties relating to the missing momentum 
correction uncertainty.

\subsubsection{Other sources of systematics}

To look at the effect of signal fraction uncertainties, we change $f_{\text {sig }}$ from the measured value by its uncertainty. For the resolution scale factor, we examine the scale factor change in a range from 1.3 to 1.7 for both muon and electron datasets. Under the new values the lifetime fits are repeated. We assign the observed lifetime changes to the systematics.

To evaluate the effect of the decay length $\left(L_{x y}\right)$ cut, we use the $B^{-} / \bar{B}^{0}$ Monte Carlo sample with the full detector simulation. We fit the Monte Carlo sample with and without the $L_{x y}>0$ cut. The difference of the fit results of these two conditions is interpreted as the systematics from the $L_{x y}$ cut.

We estimate the effect of combinatorial background shape as follows. Instead of fixing the background shape used in the final lifetime fit, we fit all the combinatorial background parameters simultaneously with the lifetimes. Very small shift of the lifetimes is observed. It is assigned to the systematic uncertainty.

Lastly we add the uncertainty of the detector alignment $2 \mu \mathrm{m}$ to the lifetime results.

A summary of all the systematic uncertainties is found in Table 4.19.

\begin{tabular}{|c|c|c|c|c|c|c|c|c|c|c|c|c|c|}
\hline$f^{* *}$ & $V_{V}$ & \multicolumn{3}{|c|}{$\begin{array}{l}\mu p_{T}\left(D^{0}\right)<8 \mathrm{GeV} / c \\
\epsilon\left(\pi_{s}\right) \\
g_{-}\end{array}$} & \multicolumn{3}{|c|}{$\begin{array}{l}\mu p_{T}\left(D^{0}\right)>8 \mathrm{GeV} / c \\
\epsilon\left(\pi_{s}\right) \\
g_{-}\end{array}$} & \multicolumn{3}{|c|}{$\begin{array}{l}e p_{T}\left(D^{0}\right)<8 \mathrm{GeV} / c \\
\epsilon\left(\pi_{s}\right) \quad g_{-}\end{array}$} & $\begin{array}{l}e p_{T}(1 \\
\epsilon\left(\pi_{s}\right)\end{array}$ & )$>8$ & $\mathrm{eV} / \mathrm{c}$ \\
\hline 24 & & & 0.780 & & & 0.895 & 0.080 & & 0.795 & 0.067 & & 0.919 & 0.066 \\
\hline & & & 741 & & & 0.8 & & & 0.15 & & & & 0.107 \\
\hline & & & 0.693 & & & 0.764 & 0.189 & & U. 68 & & & $j$ & 0.161 \\
\hline & & & 0.736 & & & 0.8 & & & 0.7 & & & & $\overline{40}$ \\
\hline & & & 746 & 0.171 & & 0.848 & 0.1 & & 0.763 & & & 874 & 0.151 \\
\hline & & & $\overline{0}$ & & & & & & 0.7 & & & & 0.107 \\
\hline 0.36 & & .110 & 0.752 & 0.121 & 1.000 & 0.838 & 0.128 & 0.153 & 0.770 & 0.109 & 1.000 & 0.870 & 0.107 \\
\hline
\end{tabular}

Table 4.17: A summary of $g_{-}$in each sample and each $D^{0}$ transverse momentum region evaluated when $f^{* *}$ and $P_{V}$ are varied. $\tau\left(B^{-}\right) / \tau\left(\bar{B}^{0}\right)$ is set to be unity in this table. 


\begin{tabular}{|c|c|c|c|c|c|c|c|c|c|}
\hline \multirow{2}{*}{$f^{* *}$} & \multirow{2}{*}{$P_{V}$} & \multicolumn{4}{|c|}{$\epsilon\left(\pi_{s}\right)$} & \multicolumn{2}{|c|}{$c \tau(\mu \mathrm{m})$} & \multirow{2}{*}{$\begin{array}{c}\text { correl. } \\
\text { coeff. }\end{array}$} & \multirow{2}{*}{$\begin{array}{l}\tau\left(B^{-}\right) \\
\tau\left(B^{0}\right) \\
\end{array}$} \\
\hline & & $\mu$ low & $\mu$ high & $e$ low & $e$ high & $B^{-}$ & $\bar{B}^{0}$ & & \\
\hline 0.24 & 0.65 & 0.673 & 0.993 & 0.707 & 1.000 & $495.0 \pm 7.8$ & $448.0 \pm 10.0$ & -0.295 & $1.105 \pm 0.034$ \\
\hline 0.36 & 0.65 & 0.673 & 0.993 & 0.707 & 1.000 & $495.6 \pm 8.6$ & $441.5 \pm 10.9$ & -0.418 & $1.123 \pm 0.040$ \\
\hline 0.48 & 0.65 & 0.673 & 0.993 & 0.707 & 1.000 & $498.1 \pm 9.8$ & $431.4 \pm 12.7$ & -0.566 & $1.155 \pm 0.051$ \\
\hline 0.36 & $\overline{0.22}$ & 0.673 & 0.993 & 0.707 & 1.000 & $494.2 \pm 8.5$ & $452.5 \pm 9.7$ & -0.340 & $1.092 \pm 0.035$ \\
\hline 0.36 & 1.00 & 0.673 & 0.993 & 0.707 & 1.000 & $496.3 \pm 8.6$ & $432.9 \pm 12.0$ & -0.463 & $1.146 \pm 0.045$ \\
\hline 0.36 & 0.65 & 0.636 & 0.944 & 0.661 & 0.933 & $495.7 \pm 8.8$ & $441.8 \pm 11.0$ & -0.440 & $1.122 \pm 0.041$ \\
\hline 0.36 & 0.65 & 0.710 & 1.000 & 0.753 & 1.000 & $495.0 \pm 8.5$ & $442.3 \pm 11.0$ & -0.410 & $1.119 \pm 0.040$ \\
\hline
\end{tabular}

Table 4.18: A summary of the lifetime fit when $f^{* *}, P_{V}, \epsilon\left(\pi_{s}\right)$ are varied. In the column $\epsilon\left(\pi_{s}\right)$ "low" and "high" mean regions of $p_{T}\left(D^{0}\right)$ lower and greater than $8 \mathrm{GeV} / c$, respectively.

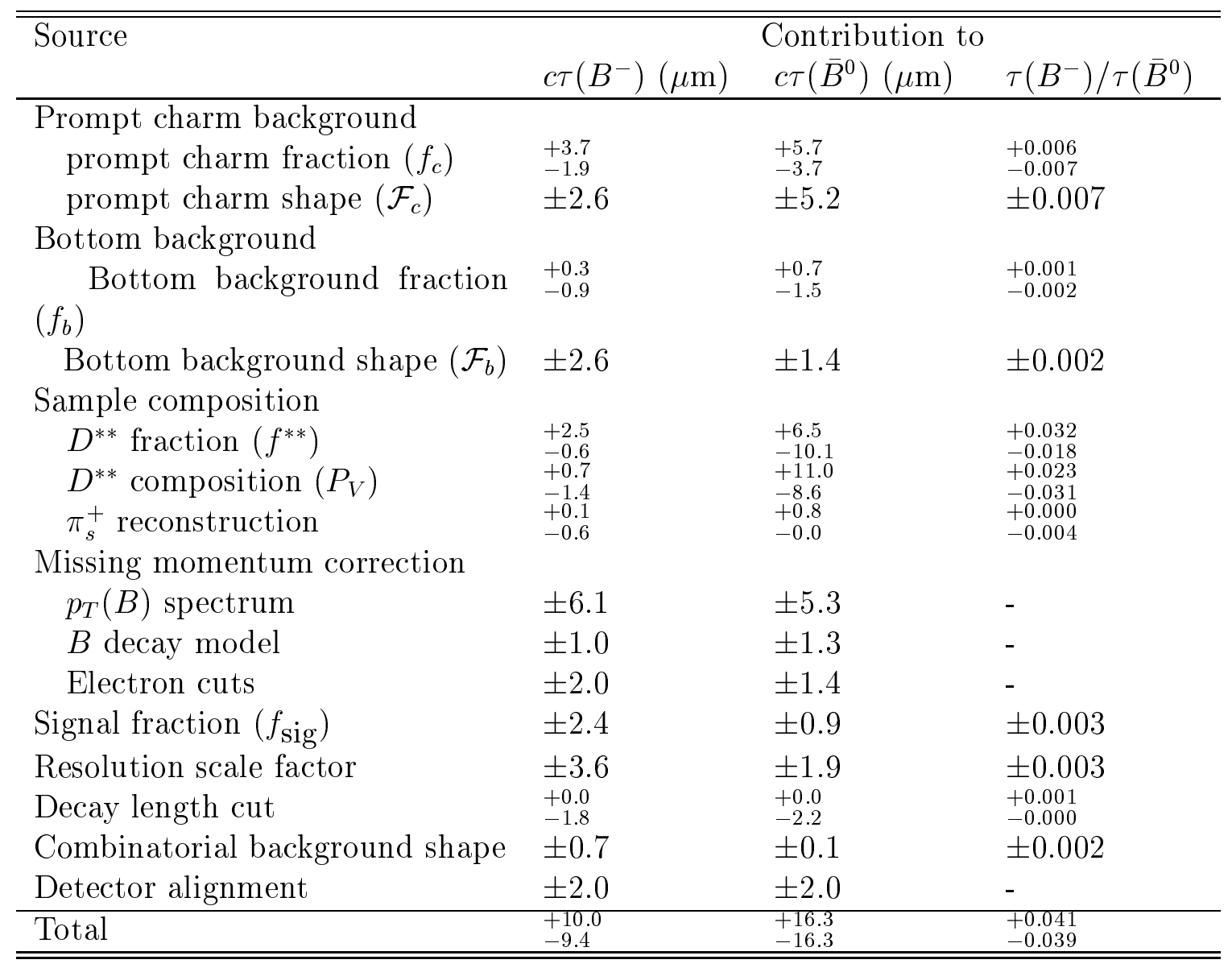

Table 4.19: A summary of the systematic uncertainties in the $B^{-} / \bar{B}^{0}$ meson lifetime measurement. 

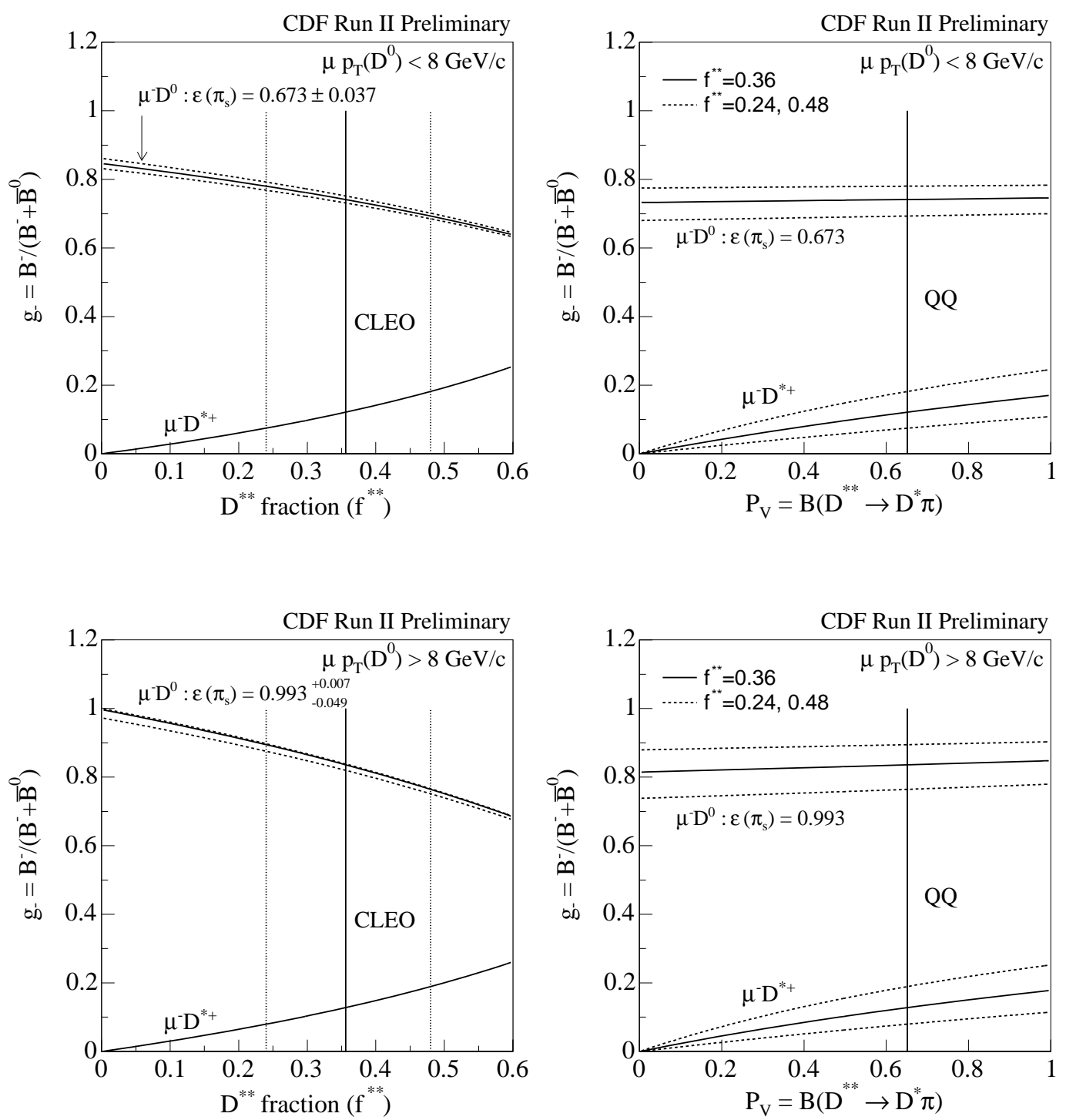

Figure 4.23: The changes of $g_{-}$as functions of $f^{* *}$ and $P_{V}$ under several conditions for muon dataset. In the left plots $P_{V}$ is set to the default value 0.651. Upper plots are for the $p_{T}\left(D^{0}\right)<8 \mathrm{GeV} / c$ region, and lower plots are for the $p_{T}\left(D^{0}\right)>8 \mathrm{GeV} / c$ region. The lifetime ratio $\tau\left(B^{-}\right) / \tau\left(\bar{B}^{0}\right)$ is set to be unity. 

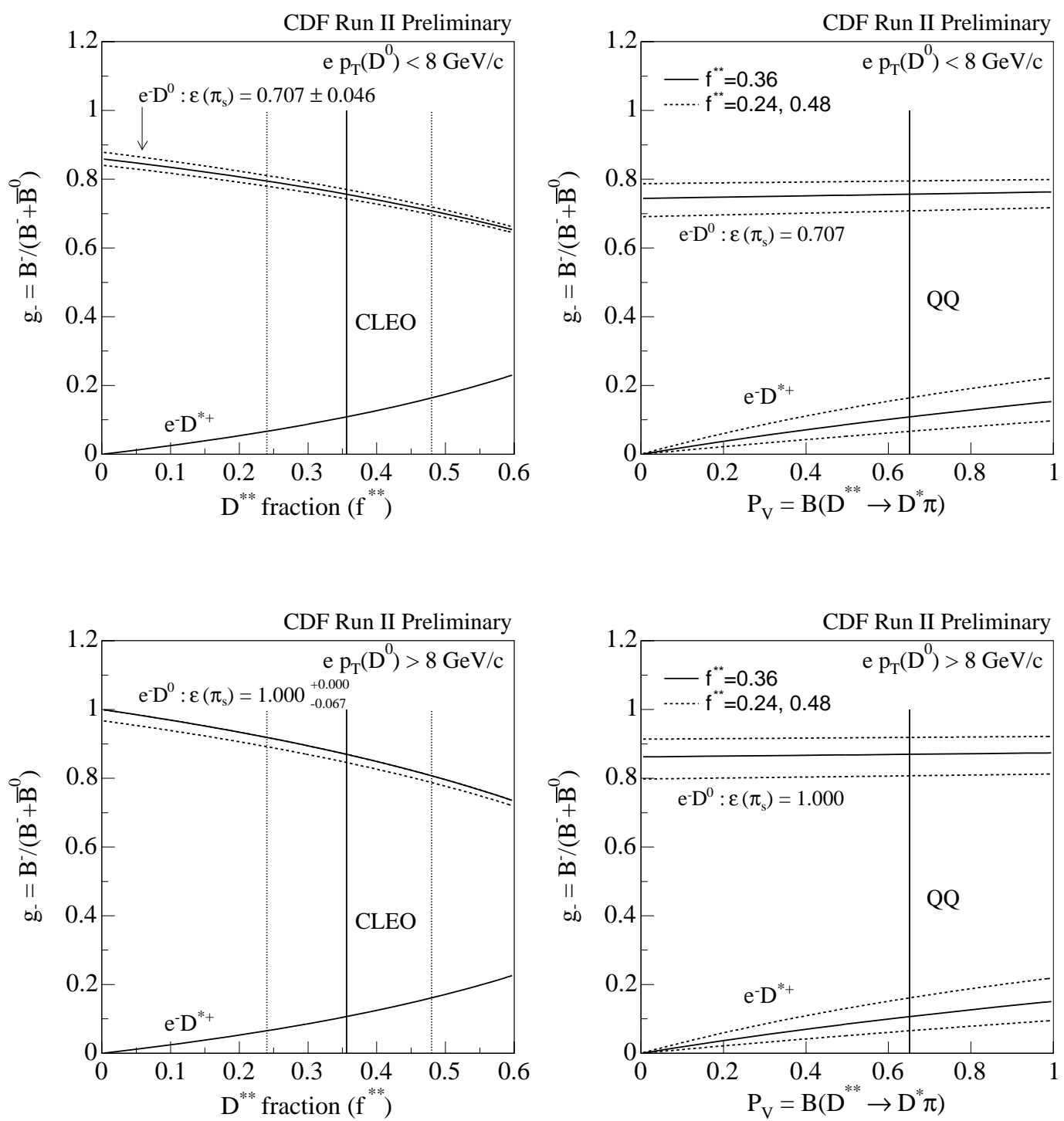

Figure 4.24: Same as the previous figures but for the electron dataset. 


\section{Chapter 5}

\section{Measurement of the $\bar{B}_{s}^{0}$ meson lifetime}

In this chapter the $\bar{B}_{s}^{0}$ meson lifetime analysis using lepton $+D_{s}^{+}$sample is described. Basically the analysis strategy is identical with the $B^{-} / \bar{B}^{0}$ lifetime analysis shown in the previous chapter, but there are some differences as listed below.

- There is no complexity from mixture of the different $B$ components in the $D_{s}^{+}$sample, that existed in the $B^{-} / \bar{B}^{0} \rightarrow \ell^{-} \bar{\nu} D^{0} X$ case.

- Data size is increased to $360 \mathrm{pb}^{-1}$.

In the following sections, basically same analysis methods with the $B^{-} / \bar{B}^{0}$ analysis are used unless specifically notified.

\section{1 $\quad \bar{B}_{s}^{0}$ Monte Carlo samples and missing mo- mentum correction}

In this section we describe about $\bar{B}_{s}^{0}$ signal Monte Carlo sample, which is needed to calculate the $K$ factor distributions and prompt charm fraction measurement. The $p_{T}(B)$ spectrum by NDE calculation [57][63] and ISGW2 semileptonic decay model implemented in EvtGen generator [59][64] are employed for the $\bar{B}_{s}^{0}$ meson generation and semileptonic decay simulation. Generated $\bar{B}_{s}^{0}$ meson is forced to decay semileptonically $\left(\bar{B}_{s}^{0} \rightarrow \ell^{-} \bar{\nu} D_{s}^{(*, * *)+}\right.$, where $\ell$ is $\mu$ or $e$ ), and decay of the $D_{s}^{+}$meson is forced into $\phi \pi^{+}$, followed by $\phi \rightarrow K^{+} K^{-}$. After the generation, full detector simulation is applied for both muon and electron events. We apply same cuts with the real data, as described in Section 3.5.2. 
As documented in Section 4.1.1, we adopt the re-weighting factor $p_{T}\left(\bar{B}_{s}^{0}\right)^{\alpha}$ to the $p_{T}\left(\bar{B}_{s}^{0}\right)$ spectrum, where $\alpha$ is measured to be -0.7320 from $J / \psi$ sample.

Also, in simulating the semileptonic decays of the $\bar{B}_{s}^{0}$ meson, we modify the fraction $f^{* *}$ of the semileptonic decays to the $D^{* *}$ mesons. It is defined as

$$
f^{* *}=\frac{\mathcal{B}\left(\bar{B}_{s}^{0} \rightarrow \ell^{-} \bar{\nu} D_{s}^{* *+}\right)}{\mathcal{B}\left(\bar{B}_{s}^{0} \rightarrow \ell^{-} \bar{\nu} D_{s}^{+} X\right)}
$$

where $X$ includes the null state. The value is not measured well for the $B_{s}^{0}$ semileptonic decays. Therefore we assume it to be the same as in the decays of non-strange $B$ mesons. The branching fractions in EvtGen correspond to the $f^{* *}$ value of 0.24 . For the $B^{-} / \bar{B}^{0}$ lifetime analysis we used $f^{* *}=0.36 \pm 0.12$. To be consistent between the two analyses, we also re-weight the $\bar{B}_{s}^{0}$ Monte Carlo sample to give $f^{* *}=0.36$.

From the $\bar{B}_{s}^{0}$ Monte Carlo sample, we calculate the $K$ factor distributions both for muon and electron datasets. The ratio $K$ has been defined to be

$$
K \equiv \frac{p_{T}\left(\ell^{-} D_{s}^{+}\right)}{p_{T}\left(\bar{B}_{s}^{0}\right)}
$$

Although the events have undergone the detector simulation and offline reconstruction, we use the particle momenta at the generator level, before smearing, to calculate the ratio. The $K$ distributions thus obtained are shown in Figure 5.1. The mean value is 0.855 (0.861), and the rms spread is 0.101 (0.098), for the muon (electron) dataset. The contributions of the decays to $D_{s}^{+}, D_{s}^{*+}$ and $D_{s}^{* *+}$ states are shown separately in Figure 5.10. 

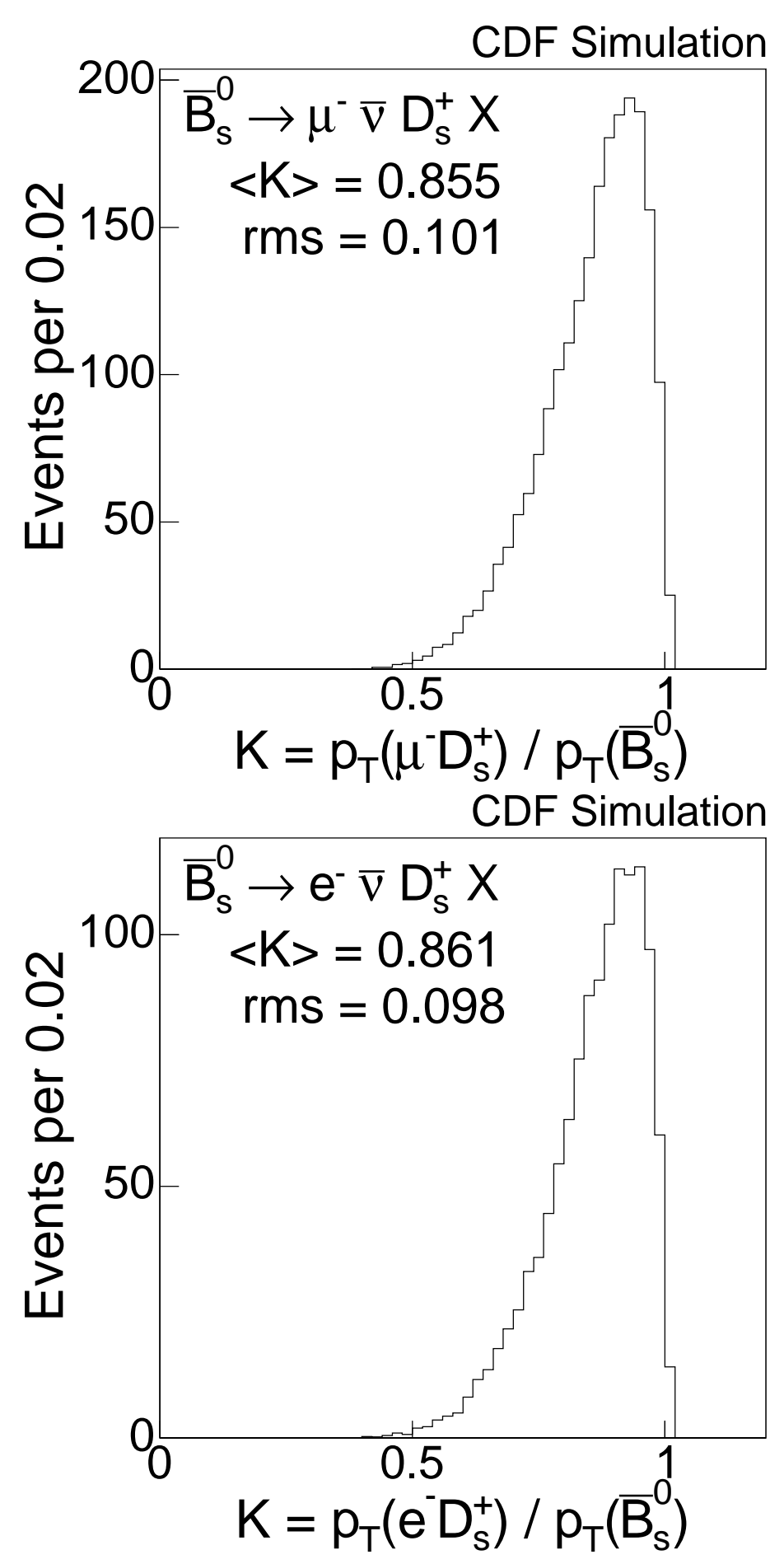

Figure 5.1: The $K$ factor distributions for the muon dataset (top) and the electron dataset (bottom). 


\subsection{Combinatorial background}

When we extract the lifetimes from the observed $c t^{*}$ distribution of the events in the signal region, defined in Table 3.3, we need to account for the contribution from the combinatorial background events under the signal mass peaks. We achieve it by including an additional term in the probability density function of the pseudo-proper decay length $c t^{*}$. We denote it by $\mathcal{F}_{\mathrm{BG}}$.

In order to determine the shape of the function $\mathcal{F}_{\mathrm{BG}}$, we use the events in the sideband of the $M\left(K^{+} K^{-} \pi^{+}\right)$distributions, and assume that they have the same $c t^{*}$ shape as the combinatorial background events under the $D_{s}^{+}$mass peak. We use the following empirical parameterization:

$$
\begin{aligned}
\mathcal{F}_{\mathrm{BG}}\left(c t^{*}\right) & =\left(1-f_{1+}-f_{+2}-f_{1-}-f_{2-}\right) G(\sigma) \\
& +f_{1+} \exp \left(-c t^{*} / \lambda_{1+}\right) \theta(+x) \otimes G(\sigma) \\
& +f_{2+} \exp \left(-c t^{*} / \lambda_{2+}\right) \theta(+x) \otimes G(\sigma) \\
& +f_{1-} \exp \left(+c t^{*} / \lambda_{1-}\right) \theta(-x) \otimes G(\sigma) \\
& +f_{2-} \exp \left(+c t^{*} / \lambda_{2-}\right) \theta(-x) \otimes G(\sigma),
\end{aligned}
$$

where $G$ is a normalized Gaussian distribution with the width $\sigma$ and centered at zero, and $\theta(x)$ is the step function. The symbol $\otimes$ means a convolution. As can bee seen, the function $\mathcal{F}_{\mathrm{BG}}$ has five components, a central Gaussian, and four smeared exponential tails, two each for positive and negative $c t^{*}$ regions, with effective lifetime parameters $\lambda$. The parameters $f$ 's are the fractions of the components. The subscripts + and - indicate the regions of $c t^{*}$ under consideration. A complete formula of $\mathcal{F}_{\mathrm{BG}}$ is given in Appendix A.2. We fit this function to the $c t^{*}$ distribution of the sideband events using a maximum likelihood method, separately for the muon and electron datasets. For the electron dataset fit, the second negative tail is removed, because here the event statistics are small and the fits tend to have difficulties converging. The results are illustrated in Figure 5.2, and the numerical values of the parameters are given in Table 5.1. 


\begin{tabular}{ccc}
\hline \hline & $\mu^{-} D_{s}^{+}$ & $e^{-} D_{s}^{+}$ \\
\hline$\sigma$ & $29.4 \pm 10.5$ & $59.4 \pm 5.8$ \\
$f_{1+}$ & $0.314 \pm 0.032$ & $0.132 \pm 0.092$ \\
$\lambda_{1+}$ & $392.6 \pm 31.2$ & $385.9 \pm 115.9$ \\
$f_{2+}$ & $0.287 \pm 0.041$ & $0.256 \pm 0.091$ \\
$\lambda_{2+}$ & $68.6 \pm 13.0$ & $141.8 \pm 47.1$ \\
$f_{1-}$ & $0.122 \pm 0.035$ & $0.141 \pm 0.031$ \\
$\lambda_{1-}$ & $180.7 \pm 29.7$ & $205.0 \pm 35.5$ \\
$f_{2-}$ & $0.263 \pm 0.045$ & 0 \\
$\lambda_{2-}$ & $45.1 \pm 11.3$ & - \\
fit probability & 0.136 & 0.567 \\
\hline \hline
\end{tabular}

Table 5.1: Fit results for the $c t^{*}$ shapes of the sideband events. Unit of $\sigma$ and $\lambda$ is $\mu \mathrm{m}$. 

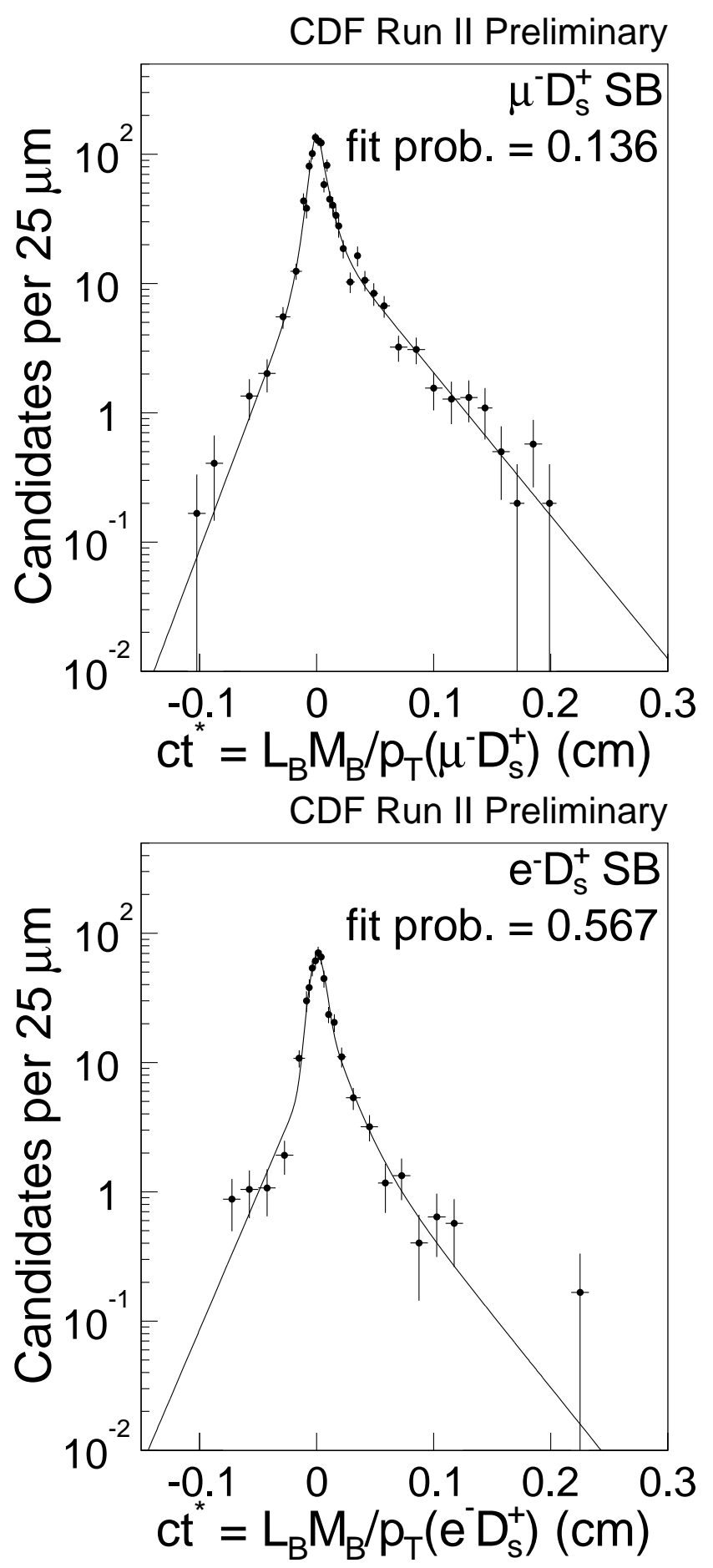

Figure 5.2: Fit results for the $c t^{*}$ distributions of the sideband events. Overlaid curves show $\mathcal{F}_{B G}$, which represent the combinatorial background shape to be used in the final lifetime fit. 


\subsection{Decay length resolution}

It is possible that the decay length resolution $\sigma_{c t^{*}}$ calculated by Vertex Fitter from estimated uncertainties in the track parameters may not exactly be a right resolution for $c t^{*}$. We account for possible incompleteness by introducing a resolution scale factor $s$, and scale $\sigma_{c t^{*}}$ to be $s \sigma_{c t^{*}}$. Determining the resolution scale factor is necessary in prior the lifetime measurement. In this section we discuss the determination of the scale factor.

As done in the $B^{-} / \bar{B}^{0}$ lifetime analysis, the resolution scale factor $s$ is determined using a sample where prompt events are enhanced. The events are selected using only loose lepton identification cuts, and the $M\left(K^{+} K^{-} \pi^{+}\right)$ window from 1900 - 1930, and from $2005-2035 \mathrm{MeV} / c^{2}$. This sample contains more fake leptons coming from the primary vertex, and accordingly an enhanced prompt peak. assuming the true $c t^{*}$ of the prompt component to be zero.

We use the following probability density function to fit the prompt enhanced sample:

$$
\begin{aligned}
\mathcal{F}_{\text {prompt }}\left(c t^{*}, \sigma_{c t^{*}}\right) & =\left(1-f_{+}-f_{-}\right) G\left(c t^{*} ; s \sigma_{c t^{*}}\right) \\
& +f_{+} \exp \left(-c t^{*} ; \lambda_{+}\right) \theta(+x) \otimes G\left(s \sigma_{c t^{*}}\right) \\
& +f_{-} \exp \left(+c t^{*} ; \lambda_{-}\right) \theta(-x) \otimes G\left(s \sigma_{c t^{*}}\right)
\end{aligned}
$$

We fit this functional form $\mathcal{F}_{\text {prompt }}$ to the $c t^{*}$ distributions of the promptenhanced samples. We find the resolution scale factor to be $s=1.59 \pm 0.05$ for the muon dataset, and $s=1.56 \pm 0.07$ for the electron dataset. The fit results are shown in Figure 5.3, and the fit parameters are given in Table 5.2.

\begin{tabular}{cccccc}
\hline \hline Sample & $s$ & $f_{+}$ & $\lambda_{+}(\mu \mathrm{m})$ & $f_{-}$ & $\lambda_{-}(\mu \mathrm{m})$ \\
\hline$\mu^{-} D_{s}^{+}$ & $1.59 \pm 0.05$ & $0.256 \pm 0.015$ & $339.6 \pm 18.4$ & $0.064 \pm 0.013$ & $168.0 \pm 24.7$ \\
$e^{-} D_{s}^{+}$ & $1.56 \pm 0.07$ & $0.234 \pm 0.024$ & $275.5 \pm 25.2$ & $0.048 \pm 0.014$ & $234.4 \pm 58.6$ \\
\hline \hline
\end{tabular}

Table 5.2: Results of the resolution scale factor determination using promptenhanced sample 

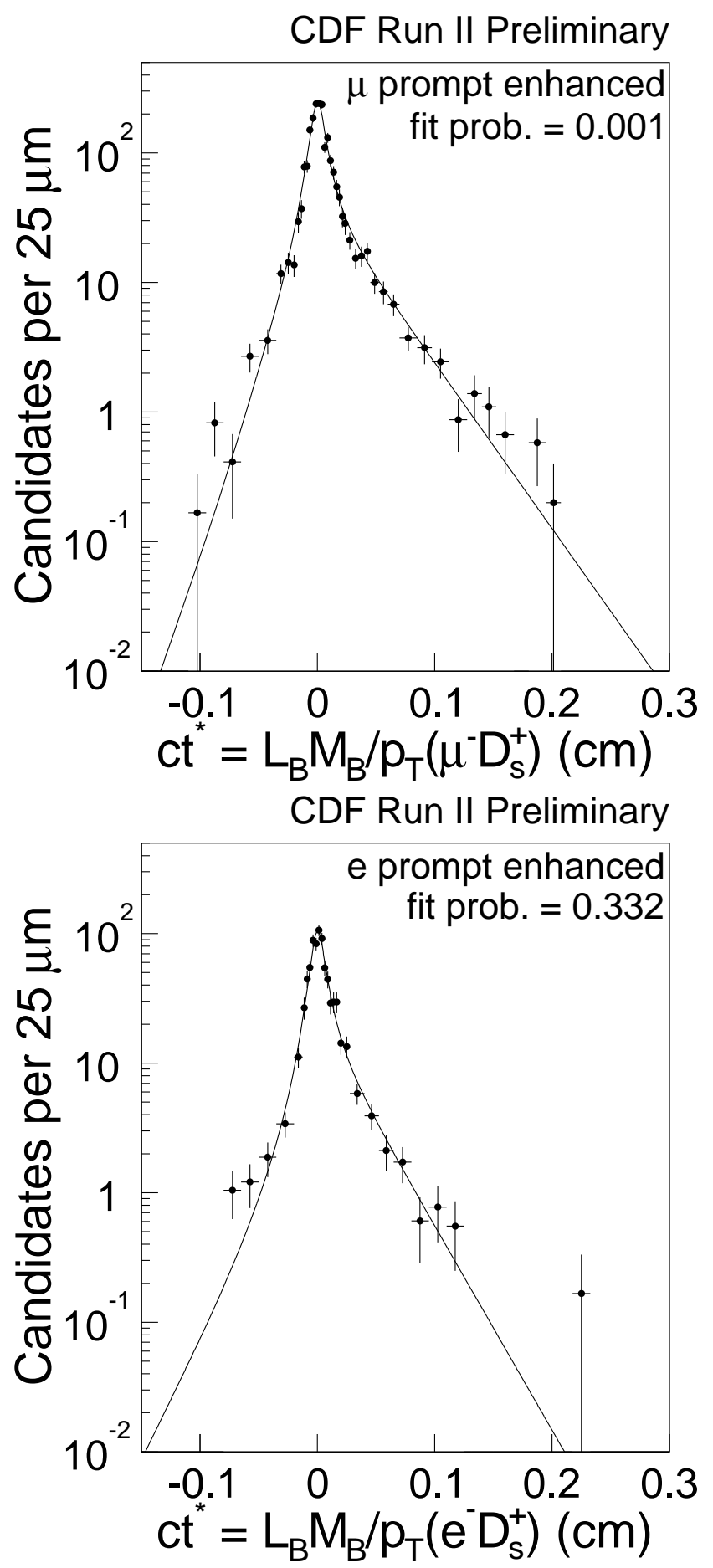

Figure 5.3: Fit results for the prompt enhanced samples when we determine the resolution scale factor. 


\subsection{Physics background}

Some physics sources other than the semileptonic decay of the $\bar{B}_{s}^{0}$ meson can produce lepton- $D_{s}^{+}$pairs that have the right charge correlation. Also, a hadron produced near the $D_{s}^{+}$meson sometimes can mimic the lepton and look like a $\ell^{-} D_{s}^{+}$pair. Here, we refer to such pairs (either the "lepton" is real or fake) as the physics background. We can think of the following two sources as possible physics backgrounds:

- promptly produced single $c$ quark and $c \bar{c}$ events.

- single $b$ and $b \bar{b}$ events, but other than the $\bar{B}_{s}^{0} \rightarrow \ell^{-} D_{s}^{+} X$ decays.

Since we know these physics backgrounds existed in the $\ell^{-} D^{0} X$ and $\ell^{-} D^{*+}$ samples, it is natural to expect that the events from the similar sources are present in the $\ell^{-} D_{s}^{+} X$ sample as well.

In this Section we discuss the treatment of such physics backgrounds. We measure the fraction of the prompt charm background from the data sample. For the other sources, we estimate them by using Monte Carlo calculations.

\subsubsection{Prompt charm background}

The first source of the physics background we consider is the promptly produced charm events (single $c$ or $c \bar{c}$ ). By "prompt" we mean that they are not the decay product of the $B$ hadrons. In such events, a real $D_{s}^{+}$meson is produced at the primary vertex. And if a track with its transverse momentum $p_{T}$ above $8 \mathrm{GeV} / c$ exists nearby, either a real lepton or a hadron faking a lepton, the pair contributes as a background.

\section{Measuring the amount}

To estimate the amount of the prompt charm background, we follow the method described in the section 4.5. It makes use of the apparent decay distance of the $D_{s}^{+}$meson measured with respect to the primary vertex. We denote the quantity by "ct.", and it is defined as follows:

$$
c t_{2} \equiv L_{x y}\left(D_{s}^{+}-P . V .\right) \frac{M\left(K^{+} K^{-} \pi^{+}\right)}{p_{T}\left(K^{+} K^{-} \pi^{+}\right)} .
$$

It is the proper decay length of the $D_{s}^{+}$meson with respect to the primary vertex. Promptly produced charm mesons are, by definition, produced at the primary vertex. Therefore, the distribution of the quantity $c t_{2}$ for those events follows an exponential function with the lifetime $c \tau\left(D_{s}^{+}\right)=147.0 \mu \mathrm{m}$. On the 
other hand, if the $D_{s}^{+}$mesons are the decay products of the $B$ mesons, the variable $c t_{2}$ should exhibit a very different, much longer, distribution, because the distance is measured from the primary to the tertiary vertex and thus involves the lifetime of the parent $B$ hadron as well as the lifetime of the $D_{s}^{+}$meson itself; it is a convolution of two exponential functions. Examples of such distributions are given in Figure C.1. The two distributions are qualitatively very different, therefore we expect to measure the relative mixture by examining the $c t_{2}$ distribution observed in the data.

We remove the $L_{x y}>0$ cut for the $D_{s}^{+}$meson in this analysis, obviously.

First we fit the $c_{2}$ distribution of the sideband events to obtain the shape for the combinatorial background events. We use the same functional form as for the combinatorial background modeling, defined in Section 5.2. (For the electron sample, we remove the second exponential tails for both the positive and negative regions.) The fit results for the sideband events are shown in Figure 5.4, bottom plots.

Then we prepare the $c t_{2}$ template distributions for the prompt and secondary charm components.

1. Prompt charm component $F_{c}\left(c t_{2}\right)$ :

It is an exponential function with the $D_{s}^{+}$meson lifetime, smeared with a resolution :

$$
F_{c}\left(c t_{2}\right)=\frac{1}{c \tau_{D_{s}^{+}}} \exp \left(-c t_{2} / c \tau_{D_{s}^{+}}\right) \otimes G\left(s_{2} \sigma_{c t_{2}}\right)
$$

where $\sigma_{c t_{2}}$ is the uncertainty in $c t_{2}$ as provided by CTVMFT, $s_{2}$ is the resolution scale factor for $\sigma_{c t_{2}}$, and $c \tau_{D_{s}^{+}}=147.0 \mu \mathrm{m}$.

2. Secondary charm component $F_{B \rightarrow D}\left(c t_{2}\right)$ :

We use a histogrammed distribution obtained from Monte Carlo events of the $\bar{B}_{s}^{0} \rightarrow \ell^{-} \bar{\nu} D_{s}^{+} X$ decays, the same sample used for the $K$ factor calculation. $^{1}$

We need to smear these distributions with a proper $c t_{2}$ resolution, and we need to obtain the resolution scale factor $s_{2}$. We measure the scale factor using the same method discussed in Section 5.3, and find it to be $s_{2}=1.46 \pm 0.04$ for the muon sample and $s_{2}=1.45 \pm 0.05$ for the electron sample. For the prompt charm function, we take the uncertainty $\sigma_{c t_{2}}$ observed for each event in the real data, and multiply it by the scale factor $s_{2}$, and use it for the smearing.

\footnotetext{
${ }^{1}$ It is possible to express the distribution in a semi-analytic form (See Appendix C). However, it is not exactly correct, and thus we use a histogrammed distribution of the Monte Carlo events.
} 


\begin{tabular}{cccc}
\hline \hline Sample & $\begin{array}{c}\text { Fit } f_{c} \\
\left(\text { all } L_{x y}\right)\end{array}$ & $\begin{array}{c}\text { Calculated } f_{c} \\
\left(L_{x y}>0\right)\end{array}$ & fit probability \\
\hline$\mu^{-} D_{s}^{+}$ & $+3.9_{-3.9}^{+4.9} \%$ & $+3.6_{-3.6}^{+4.5} \%$ & 0.1161 \\
$e^{-} D_{s}^{+}$ & $-9.2 \pm 7.1 \%$ & $-9.2 \pm 7.1 \%$ & 0.0001 \\
\hline \hline
\end{tabular}

Table 5.3: Fraction of prompt charm background obtained from fit to $c t_{2}$ distribution.

For the $B \rightarrow D$ function, we smear the generator-level quantity of $c t_{2}$ by an average (event independent) resolution. It is obtained as the mean value of the sideband-subtracted $\sigma_{c t^{*}}$ distribution of the events in the signal region, multiplied by the scale factor: $s_{2}\left\langle\sigma_{c t_{2}}\right\rangle=1.46 \times 35.5=51.8 \mu \mathrm{m}$ for the muon sample and $1.45 \times 38.5=55.8 \mu \mathrm{m}$ for the electron sample.

Now we determine the fraction of the prompt charm component. The $L_{x y}>$ 0 cut is disabled. We use the following probability density function:

$$
F\left(c t_{2}\right)=f_{\text {Sig }}\left\{f_{c} F_{c}\left(c t_{2}\right)+\left(1-f_{c}\right) F_{B \rightarrow D}\left(c t_{2}\right)\right\}+\left(1-f_{\mathrm{sig}}\right) F_{\mathrm{BG}}\left(c t_{2}\right)
$$

where $F_{\mathrm{BG}}$ is the combinatorial background function, $F_{c}$ and $F_{B \rightarrow D}$ are the prompt and secondary charm functions, respectively. The fraction $f_{c}$ is the fraction of the prompt charm component, and it is what we want to determine from real data. It is the only free parameter in the fit. The fraction returned from the fit is for the all region of $c t_{2}$. Since we apply the cut $L_{x y}>0.0$ in the end, we calculate the fraction $f_{c}$ corresponding to that region. The fit results are summarized in Table 5.3, and are also shown in Figure 5.4.

Overall the results are consistent with the $B^{-} / \bar{B}^{0}$ lifetime analysis (Section 4.5.2) but here the uncertainties are larger because of the smaller statistics. Since a negative $f_{c}$ is not physical, we interpret $f_{c}$ for the electron sample as $f_{c}=0.0{ }_{-0.0}^{+7.1} \%$.

\section{$c t^{*}$ shape of prompt charm events}

To account for the prompt charm background when we extract the $B_{s}^{0}$ meson lifetime, we need to know the distribution of the " $B$ meson decay length" $c t^{*}$ of those prompt charm events. The decay length $c t^{*}$ is determined using the distance between the primary vertex and the $B$ meson decay vertex. Since the $B$ meson decay vertex is obtained as the intersection of the "lepton" track and the $D_{s}^{+}$meson flight direction, the position of the " $B$ vertex" for those prompt charm events depends on the nature of the "lepton" track.

We can classify them into the following two categories: 
1. The "lepton" is produced at the primary vertex.

The particle can be produced in the fragmentation process $c \rightarrow D_{s}^{+} X$, or come from the remaining part of the event, unrelated to the hard collision.

Since the $D_{s}^{+}$meson is produced at, and points back to, the primary vertex, and the "lepton" is produced at the same point, they intersect at the primary vertex. Therefore, these events give $c t^{*}=0$, modulo detector resolution.

2. The "lepton" is a decay product of the other charm hadron in the event.

Namely, we have a process

$$
\bar{p} p \rightarrow c \bar{c} X, \quad c \rightarrow D_{s}^{+} X^{\prime}, \quad \bar{c} \rightarrow \bar{D} X^{\prime \prime}, \quad \bar{D} \rightarrow \text { "lepton" }+X^{\prime \prime \prime} .
$$

The $D_{s}^{+}$meson is produced at the primary vertex, as in Category 1 . However, the "lepton" is a decay product of a weakly-decaying charmed hadron $\bar{D}$, either $\bar{D}^{0}, D^{-}, D_{s}^{-}$, or $\bar{\Lambda}_{c}^{-}$, and can be displaced.

The "lepton" points back to the primary vertex on the average, but not on an event-by-event basis.

They can, in principle, have $c t^{*} \neq 0$.

The true $c t^{*}$ value of the Category-1 events is zero, thus an observed $c t^{*}$ shape can be modeled with a Gaussian distribution centered at zero, with $\sigma$ being the $c t^{*}$ resolution.

$$
\mathcal{F}_{c}^{\text {zero }}=G\left(c t^{*} ; s \sigma_{c t^{*}}\right)
$$

where we use the same scale factor $s$ determined in Section 5.3. The uncertainty $\sigma_{c t^{*}}$ is calculated for each event with CTVMFT.

The $c t^{*}$ shape of the Category-2 events is estimated from a generic $c \bar{c}$ Monte Carlo sample generated with Pythia [67]. The Pythia generator is set for generic $2 \rightarrow 2$ QCD processes (MSEL $=1$ ), without specifying the parton subprocess or species, so as to allow for possible "gluon-splitting" $c \bar{c}$ events. After an event is generated, we keep it if it contains a charm quark, $c$ or $\bar{c}$. No detector simulation is applied for this sample. Then we select "lepton"- $D_{s}^{+}$ pairs, and re-decay the $D_{s}^{+}$meson to the final state $\phi \pi^{+}, \phi \rightarrow K^{+} K^{-}$. Then we apply the following kinematic cuts on the generator-level quantities:

- A right-sign "lepton"- $D_{s}^{+}$pair.

- $p_{T}\left(\ell^{-}\right)>8 \mathrm{GeV} / c$

- $\left|\eta\left(\ell^{-}\right)\right|<0.7$ 
- $p_{T}\left(K^{ \pm}\right)>1.2 \mathrm{GeV} / c$

- $p_{T}\left(\pi^{+}\right)>0.9 \mathrm{GeV} / c$

- $\left|\eta\left(K^{ \pm}, \pi^{+}\right)\right|<1.1$

- $\Delta R\left(\ell^{-}-K^{ \pm}, \pi^{+}\right)<0.8$

- $3.0<M\left(\ell^{-} D_{s}^{+}\right)<5.4 \mathrm{GeV} / c^{2}$

The "lepton" does not have to be real, rather we count all particles.

We then calculate the pseudo-proper decay length $c t^{*}$ from the momentum of the pair, $p_{T}\left(\ell^{-} D_{s}^{+}\right)$, and the intersection of the "lepton" track and the $D_{s}^{+}$flight direction. The resultant $c t^{*}$ distributions are shown in Figure 5.5 for $c \bar{c} \rightarrow D_{s}^{+} \ell^{-}$and $c \bar{c} \rightarrow D_{s}^{+} h^{-}$events, after including a constant (event independent) resolution smearing with $s \sigma_{c t^{*}}=83 \mu \mathrm{m}$. It is the average value of $\sigma_{c t^{*}}$ observed for the sideband-subtracted events in the signal region of the real data, multiplied by the scale factor $s$ determined earlier. To include the shape in the probability density function for the lifetime determination, it is more convenient to use an analytic form, and we use the following parameterization:

$$
\begin{aligned}
\mathcal{F}_{c}^{\text {non-zero }}\left(c t^{*}\right) & =\left(1-f_{+}-f_{-}\right) G\left(c t^{*} ; \sigma\right) \\
& +f_{+} \exp \left(-c t^{*} / \lambda_{+}\right) \theta(+x) \otimes G(\sigma) \\
& +f_{-} \exp \left(+c t^{*} / \lambda_{-}\right) \theta(-x) \otimes G(\sigma)
\end{aligned}
$$

Table 5.4 summarizes the numerical values of the the parameters in $\mathcal{F}_{c}^{\text {non-zero }}$.

One can notice that the shape is mostly "prompt", with small tails, even though the "lepton" here is a decay product of a charm meson and originates from a secondary vertex. It can be understood as follows. In order for an event to have a finite lifetime, the "lepton" track has to have a finite impact parameter with respect to the primary vertex. It can happen, because the charm mesons have finite lifetimes and masses. However, the "lepton" is required to have a momentum above $8 \mathrm{GeV} / c$, and it is much larger than the parent charm hadron masses. Therefore, the lepton momentum direction is not very different from the parent charm hadron direction, thus giving a small impact parameter with respect to the primary vertex.

We have measured the total amount of the prompt charm background in the real data, using the $c t_{2}$ distribution. But we do not know its composition in terms of the two categories considered here. However, from the $B^{-} / \bar{B}^{0}$ meson lifetime analysis, we have a circumstantial evidence that the events in Category 1 (namely fake leptons coming from the primary vertex) dominate. Therefore, we adopt Category 1 to represent the prompt charm background, and consider the $c t^{*}$ shape of Category 2 as an alternative and assign the difference as a systematic uncertainty. 
Note that these are the two extreme cases, and the true shape of the prompt background lies somewhere in between.

\begin{tabular}{lccccc}
\hline \hline Type & $\sigma$ & $f_{+}$ & $\lambda_{+}$ & $f_{-}$ & $\lambda_{-}$ \\
\hline$c \bar{c} \rightarrow \ell^{-} D_{s}^{+}$ & $69.9 \pm 19.3$ & $0.335 \pm 0.123$ & $344.0 \pm 129.2$ & $0.279 \pm 0.145$ & $174.9 \pm 821.1$ \\
$c \bar{c} \rightarrow h^{-} D_{s}^{+}$ & $114.5 \pm 9.2$ & $0.113 \pm 0.039$ & $457.1 \pm 134.7$ & $0.061 \pm 0.024$ & $968.4 \pm 938.6$ \\
\hline \hline
\end{tabular}

Table 5.4: The $\mathcal{F}_{c}^{\text {non-zero }}$ parameters. Unit of $\sigma$ and $\lambda_{ \pm}$is $\mu \mathrm{m}$. 

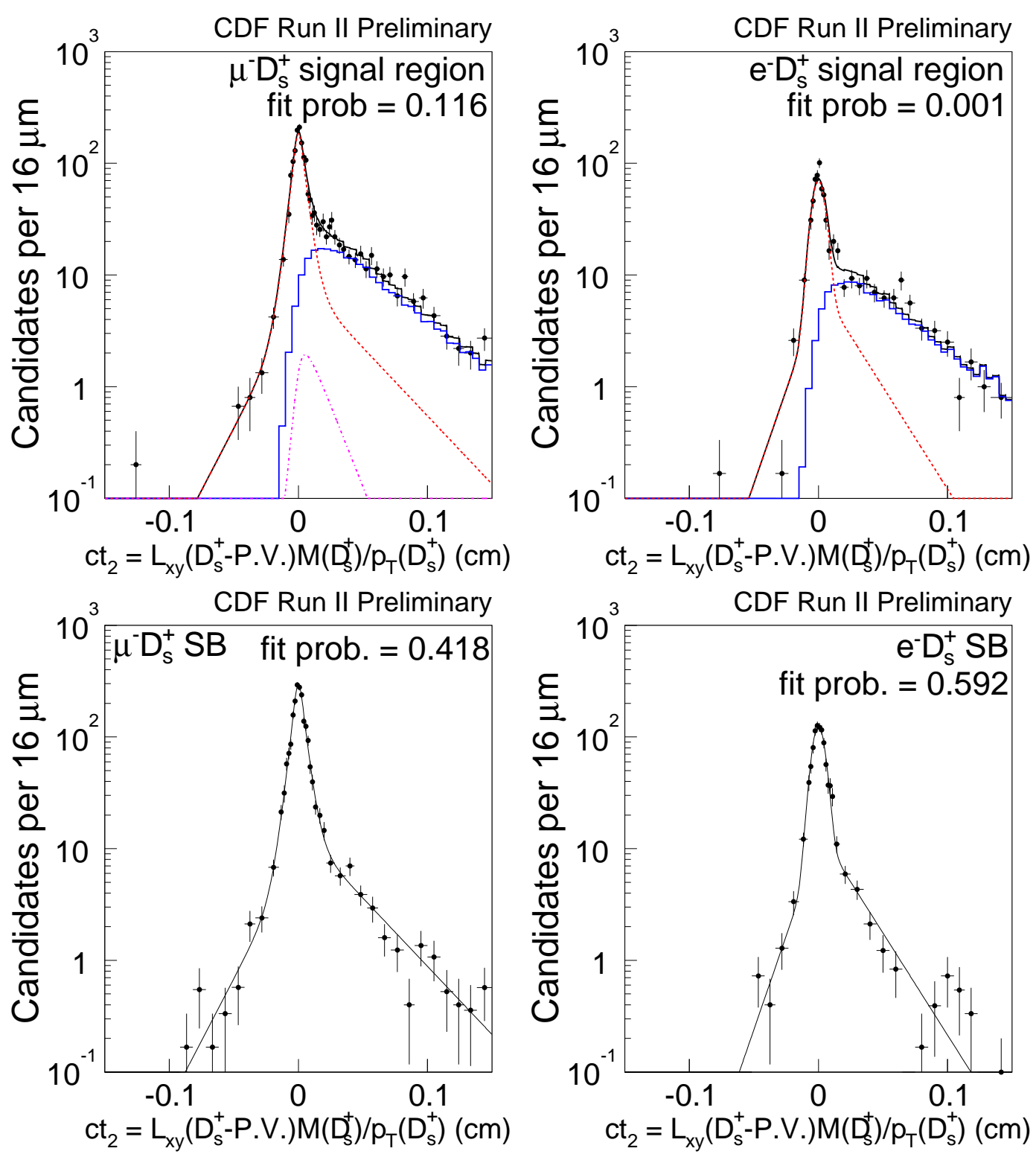

Figure 5.4: Results of the $f_{c}$ fit for muon and electron datasets. Top plots are fit results for the signal region. Bottom plots are for the sideband fit results to determine the combinatorial background shape. In the top plot, green and blue curve show the prompt charm and $\bar{B}_{s}^{0}$ templates, respectively. Red curve describes the combinatorial background shape. 

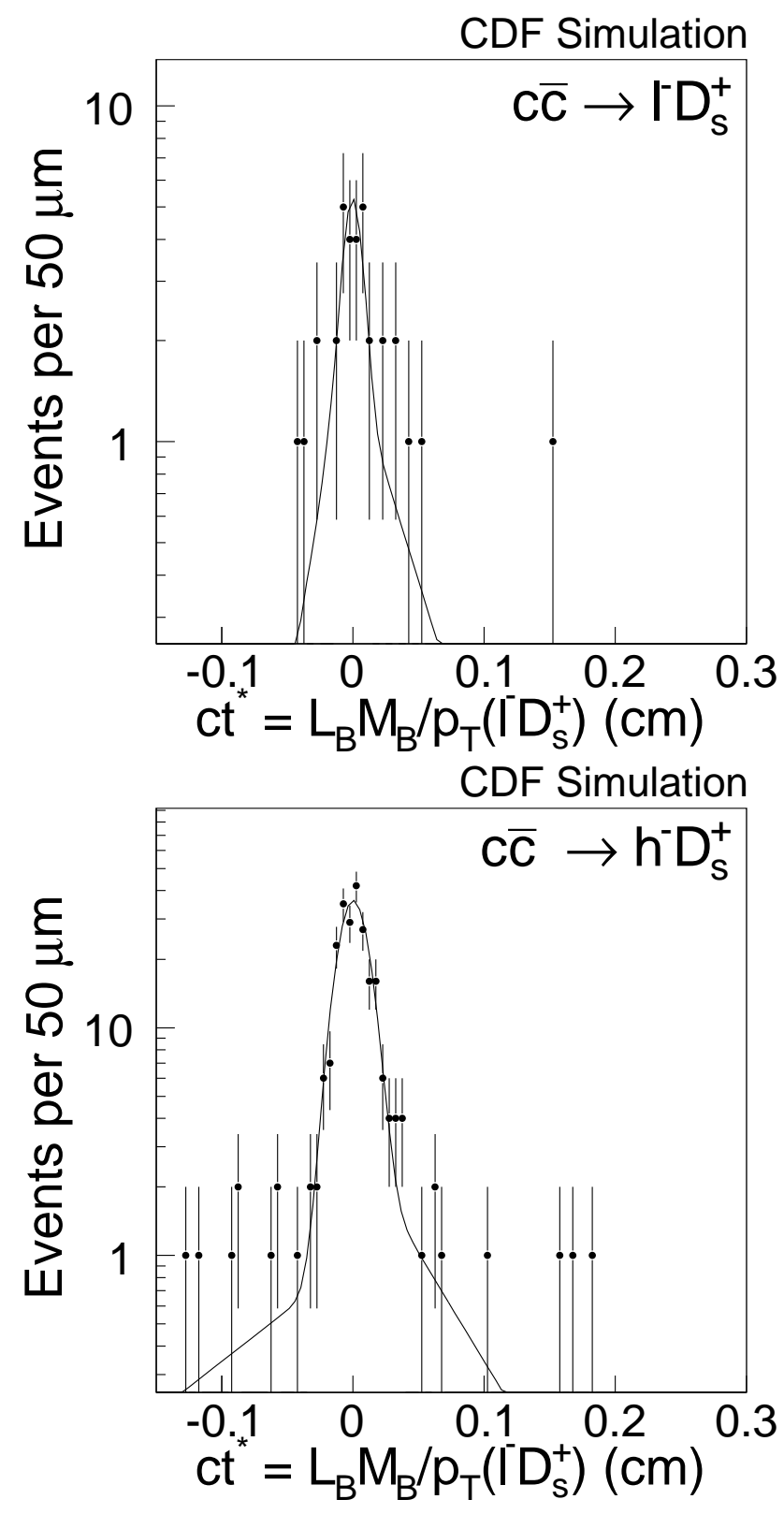

Figure 5.5: Non-zero $c t^{*}$ distributions for prompt $c \bar{c}$ events. 


\subsubsection{Bottom background}

We expect that real, right-sign $\ell^{-} D_{s}^{+}$pairs are produced in the decays of the $B$ hadrons other than the semileptonic decay of the $\bar{B}_{s}^{0}$ meson $\left(\bar{B}_{s}^{0} \rightarrow\right.$ $\left.\ell^{-} \bar{\nu} D_{s}^{+} X, \ell=e, \mu\right)$. They dominantly come from the $B$ meson decays to charm pairs, $B^{-} / \bar{B}^{0} \rightarrow D_{s}^{-} D X$. Here we call such events as "bottom background". Since they can not be removed completely, it is necessary to estimate their amount and account for them in the lifetime fits.

To estimate the contribution from these processes, we rely on a Monte Carlo calculation. After the event generation, the sample is processed through a full detector simulation. We generate the three bottom background processes listed below :

- $B^{+} / B^{0} \rightarrow D_{s}^{+} \bar{D} X, \bar{D} \rightarrow \ell^{-} \bar{\nu} X$

- $\bar{B}_{s}^{0} \rightarrow D_{s}^{+} D_{s}^{-} X, D_{s}^{-} \rightarrow \ell^{-} \bar{\nu} X$

- $\bar{B}_{s}^{0} \rightarrow \tau^{-} \bar{\nu} D_{s}^{+} X, \tau^{-} \rightarrow \ell^{-} \bar{\nu} \nu_{\tau}$

as well as the signal process.

We apply the same kinematic and selection cuts as in the real data, and count the numbers of events. We apply the $p_{T}(B)^{\alpha}$ weighting as described in Section 4.1.1. Table 5.5 summarizes the weighted number of events coming from each source. Please note that the amount of the background process 1 depends on the relative fraction of the $\bar{B}_{s}^{0}$ meson and the non-strange $\bar{B}$ mesons produced in the fragmentation of the $b$ quark. The Pythia generator assumes $f_{s} /\left(f_{u}+f_{d}\right)=0.3 / 2$. Although we do not really know this quantity at the Tevatron, we assume the value in Pythia. And we consider an alternative value estimated from real data, and treat it as a systematic uncertainty.

From the Table, we find

$$
f_{b}^{\prime}=\frac{N_{b}}{N_{\mathrm{sl}}}= \begin{cases}7.0 \pm 0.2 \% & \text { for } \mu^{-} D_{s}^{+} \text {sample } \\ 3.5 \pm 0.1 \% & \text { for } e^{-} D_{s}^{+} \text {sample }\end{cases}
$$

where $N_{b}$ is the total number of bottom background events, and $N_{\mathrm{Sl}}$ is number of the semileptonic $\bar{B}_{s}^{0}$ decay signals. From $f_{b}^{\prime}$ and $f_{c}$ we extract the total bottom background fraction $f_{b}$ in each sample.

$$
f_{b}=\frac{N_{b}}{N_{\mathrm{Sl}}+N_{c}+N_{b}}=\frac{f_{b}^{\prime}}{1+f_{b}^{\prime}}\left(1-f_{c}\right)= \begin{cases}6.3 \pm 0.3 \% & \text { for } \mu^{-} D_{s}^{+} \text {sample } \\ 3.4 \pm 0.3 \% & \text { for } e^{-} D_{s}^{+} \text {sample }\end{cases}
$$

One may notice that the numbers are different between the electron and muon samples. It is because the electron identification cuts implicitly require that the electron to be isolated within the three CEM towers in a wedge. It 


\begin{tabular}{ll|cc|cc}
\hline \hline Process & & \multicolumn{2}{|c|}{ muon } & \multicolumn{2}{c}{ electron } \\
& & $N_{\text {events }}$ & fraction & $N_{\text {events }}$ & fraction \\
\hline Signal & $B_{s}^{0} \rightarrow \ell^{-} \bar{\nu} D_{s}^{+} X$ & $283.18 \pm 2.90$ & 0.934 & $264.40 \pm 2.83$ & 0.966 \\
BG 1 & $B^{+} / B^{0} \rightarrow D_{s}^{+} \bar{D} X$ & $12.77 \pm 0.16$ & 0.042 & $5.98 \pm 0.11$ & 0.022 \\
BG 2 & $\bar{B}_{s}^{0} \rightarrow D_{s}^{+} D_{s}^{-} X$ & $2.92 \pm 0.29$ & 0.010 & $0.70 \pm 0.14$ & 0.003 \\
BG 3 & $\bar{B}_{s}^{0} \rightarrow \tau \bar{\nu} D_{s}^{+} X$ & $4.18 \pm 0.35$ & 0.014 & $2.49 \pm 0.28$ & 0.009 \\
\hline \hline
\end{tabular}

Table 5.5: Estimate of bottom backgrounds using Monte Carlo calculation. $N_{\text {events }}$ is the number of events which survive all cuts, weighted for the $p_{T}^{B}$ spectra and the branching fractions of forced decay modes.

\begin{tabular}{ccc}
\hline \hline & $\lambda_{+}(\mu \mathrm{m})$ & $\sigma(\mu \mathrm{m})$ \\
\hline Muon & $638.8 \pm 12.9$ & $85.8 \pm 3.8$ \\
Electron & $599.5 \pm 15.7$ & $106.3 \pm 12.7$ \\
\hline \hline
\end{tabular}

Table 5.6: $c t^{*}$ shape of the bottom background events.

has been known that the efficiencies of the cuts are a factor of two or three different between the electrons produced in the semileptonic decays of the $B$ meson and the $D$ meson, $\bar{B} \rightarrow \ell^{-} \bar{\nu} X$ vs. $\bar{c} \rightarrow \bar{D} X^{\prime} \rightarrow \ell^{-} \bar{\nu} X^{\prime \prime}$. (See Figure 11 in [68].) The efficiency is lower for the electrons from the charm hadron decays. It is because their masses are substantially smaller than the $B$ hadron mass, and the probability that the particle "X" hits the same calorimeter cell as the electron is much higher. Here, the background produces an additional charm hadron, and the probability could be even higher. In any case the numbers obtained here are compatible with those in [68].

Their decay time distributions are essentially an exponential distribution, reflecting the $B$ meson lifetimes. However, the lepton- $D_{s}^{+}$pair carries a different (smaller) fraction of the parent $B$ meson momentum than the signal, and so the apparent lifetime is longer. We estimate the shape of the bottom backgrounds also using the Monte Carlo sample.

We smear $c t^{*}$ from generator level information with a realistic resolution measured from the data.

The $c t^{*}$ distributions thus obtained for the bottom backgrounds are shown in Figure 5.6. We parameterize the distribution of the sum of the all bottom 
background processes with the following function:

$$
\mathcal{F}_{b}\left(c t^{*}\right)=\frac{1}{\lambda_{+}} \exp \left(-c t^{*} / \lambda_{+}\right) \theta(x) \otimes G(\sigma)
$$

The fit results are shown as curves in Figure 5.6. The numerical values of the parameters are given in Table 5.6. The functional shape, as well as the parameters, are used for the signal lifetime fits. The shapes of the three different background processes are considered when evaluating the systematic uncertainties.
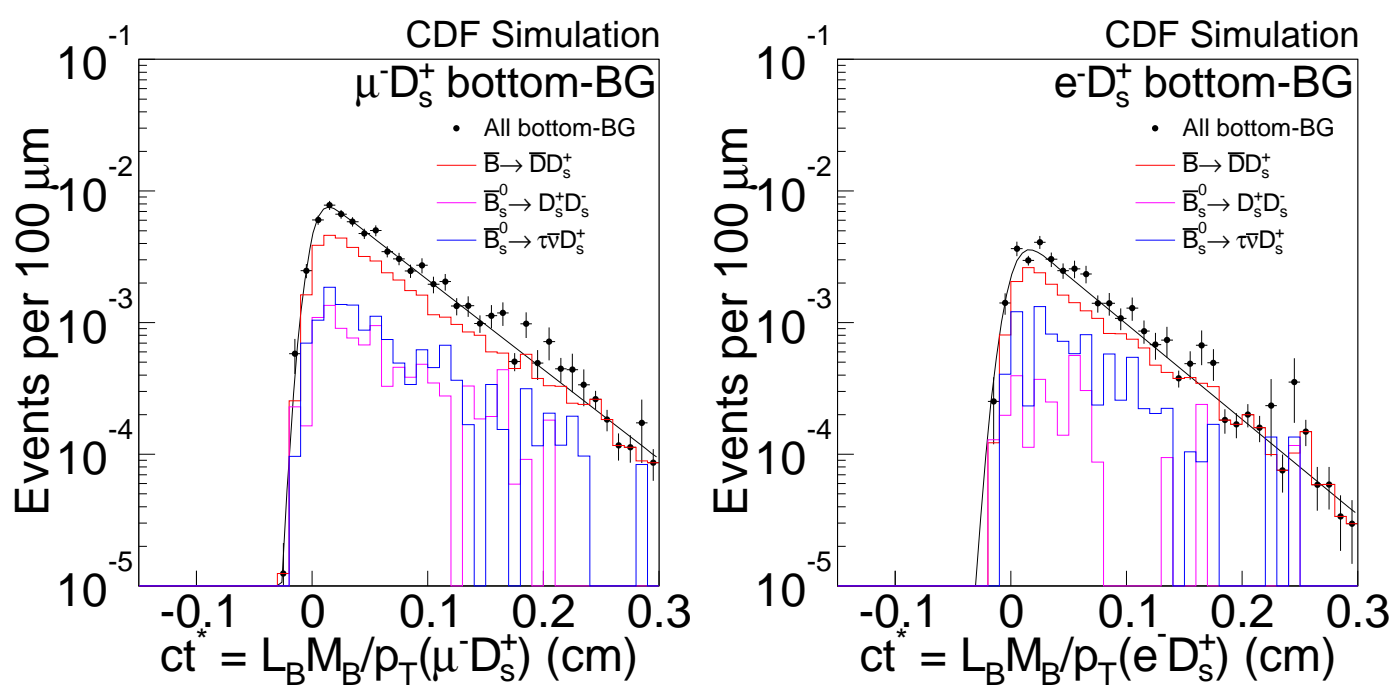

Figure 5.6: The $c t^{*}$ distributions for bottom background events obtained from the Monte Carlo simulation for muon and electron datasets. Colored histograms show contributions from each processes, and points show sum of all the contributions. Overlaid curves are fit results for the total background shapes and represent the bottom background template functions. 


\subsection{Lifetime extraction}

Similarly to the previous $B^{-} / \bar{B}^{0}$ analysis, we define the probability density function for the semileptonic $\bar{B}_{s}^{0}$ decays as follows.

$$
\mathcal{F}_{\bar{B}_{s}^{0}}\left(c t^{*}, \sigma_{c t^{*}} ; c \tau, s\right)=\operatorname{Exp}\left(-c t^{*} ; c \tau / K\right) \otimes H(K) \otimes G\left(s \sigma_{c t^{*}}\right)
$$

where $H(K)$ denotes the $K$ factor distribution, $G(\sigma)$ means Gaussian smearing with the resolution of $\sigma$. An exact formulation of $\mathcal{F}_{\bar{B}_{s}^{0}}$ is shown in Appendix A.1. Then we define the event probability density function including background functions as follows.

$$
P\left(c t^{*}, \sigma_{c t^{*}}\right)=f_{\text {sig }}\left\{\left(1-f_{c}-f_{b}\right) \mathcal{F}_{\bar{B}_{s}^{0}}+f_{c} \mathcal{F}_{c}+f_{b} \mathcal{F}_{b}\right\}+\left(1-f_{\text {sig }}\right) \mathcal{F}_{\mathrm{BG}}
$$

Then the following combined likelihood is formed both for muon and electron datasets.

$$
L_{x}=\prod_{i} P_{i}(X=\mu, e)
$$

where the product is taken over all the events in the signal region of each dataset. At the lifetime fit we actually minimize the negative log likelihood $-2 \log L$. Firstly we perform a separate fit each for muon and electron dataset, maximizing the likelihood $L_{\mu}$ or $L_{e}$. Following are the results of muon, electron separate lifetime fits.

$$
c \tau\left(\bar{B}_{s}^{0}\right)=\left\{\begin{array}{l}
411.6 \pm 20.7 \mu \mathrm{m} \text { (muon) } \\
418.4 \pm 27.8 \mu \mathrm{m} \text { (electron) }
\end{array}\right.
$$

Here the uncertainties are statistical only. The $c t^{*}$ distributions and fit results for each sample are shown in Figure 5.7.

Then we form the combined likelihood using both the muon and electron dataset, and perform the combined lifetime fit.

$$
L_{\text {combined }}=L_{\mu} \cdot L_{e}
$$

As a result of the muon and electron combined fit, we obtain

$$
c \tau\left(\bar{B}_{s}^{0}\right)=414.0 \pm 16.6 \mu \mathrm{m}
$$

The $c t^{*}$ distribution and combined fit functions are shown in Figure 5.8.

In Table 5.7, fit probabilities are summarized for all the fits. 


\begin{tabular}{cc}
\hline \hline Dataset & fit probability \\
\hline$\mu$ only & 0.1818 \\
$e$ only & 0.5359 \\
Combined & 0.2820 \\
\hline \hline
\end{tabular}

Table 5.7: Fitting probabilities calculated from each fit result.
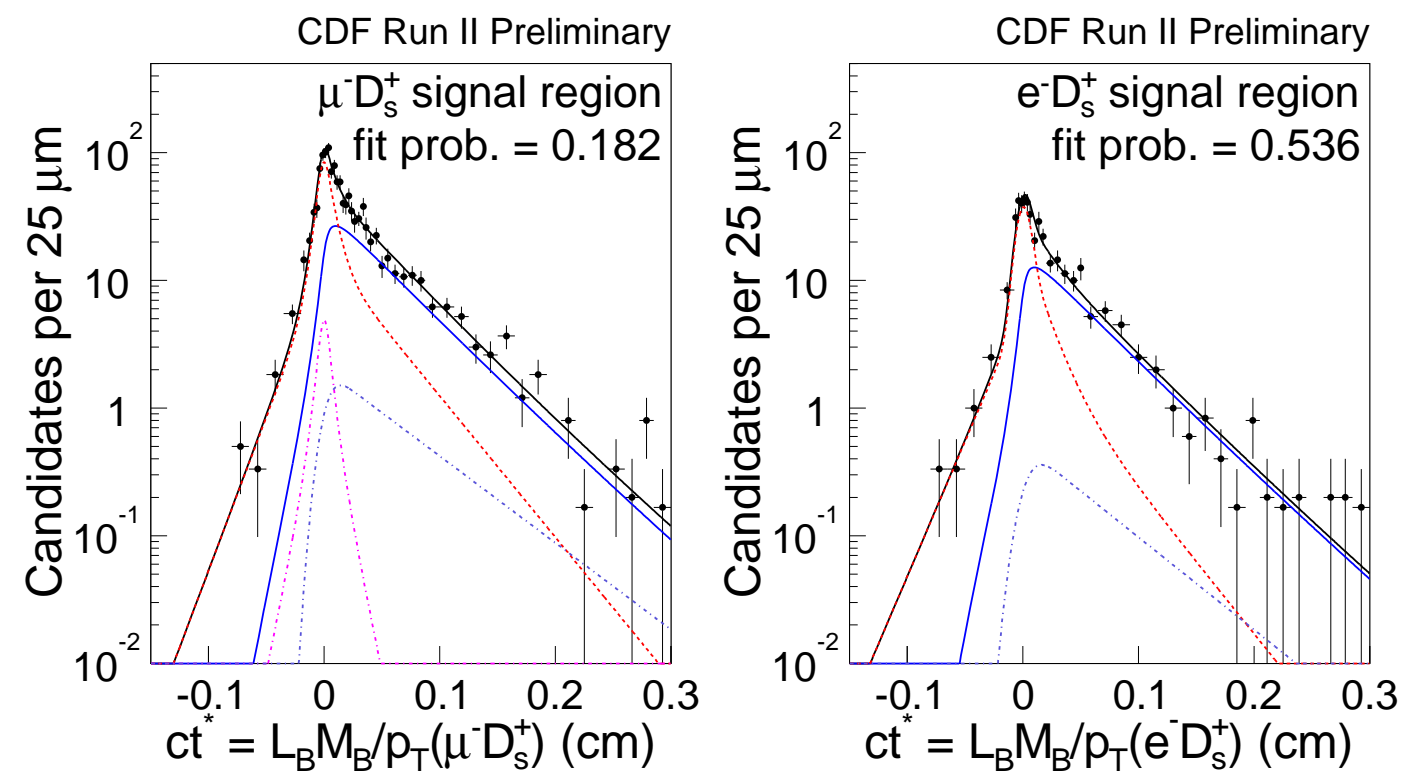

Figure 5.7: The $c t^{*}$ distributions and lifetime fit results. Left plot is for the muon, and right plot is for the electron dataset. Overlaid curves are the fit functions. Blue curves show the semileptonic $\bar{B}_{s}^{0}$ signal components, red curves are the combinatorial background function. Dashed green and blue curves show the charm and bottom background components, respectively. 


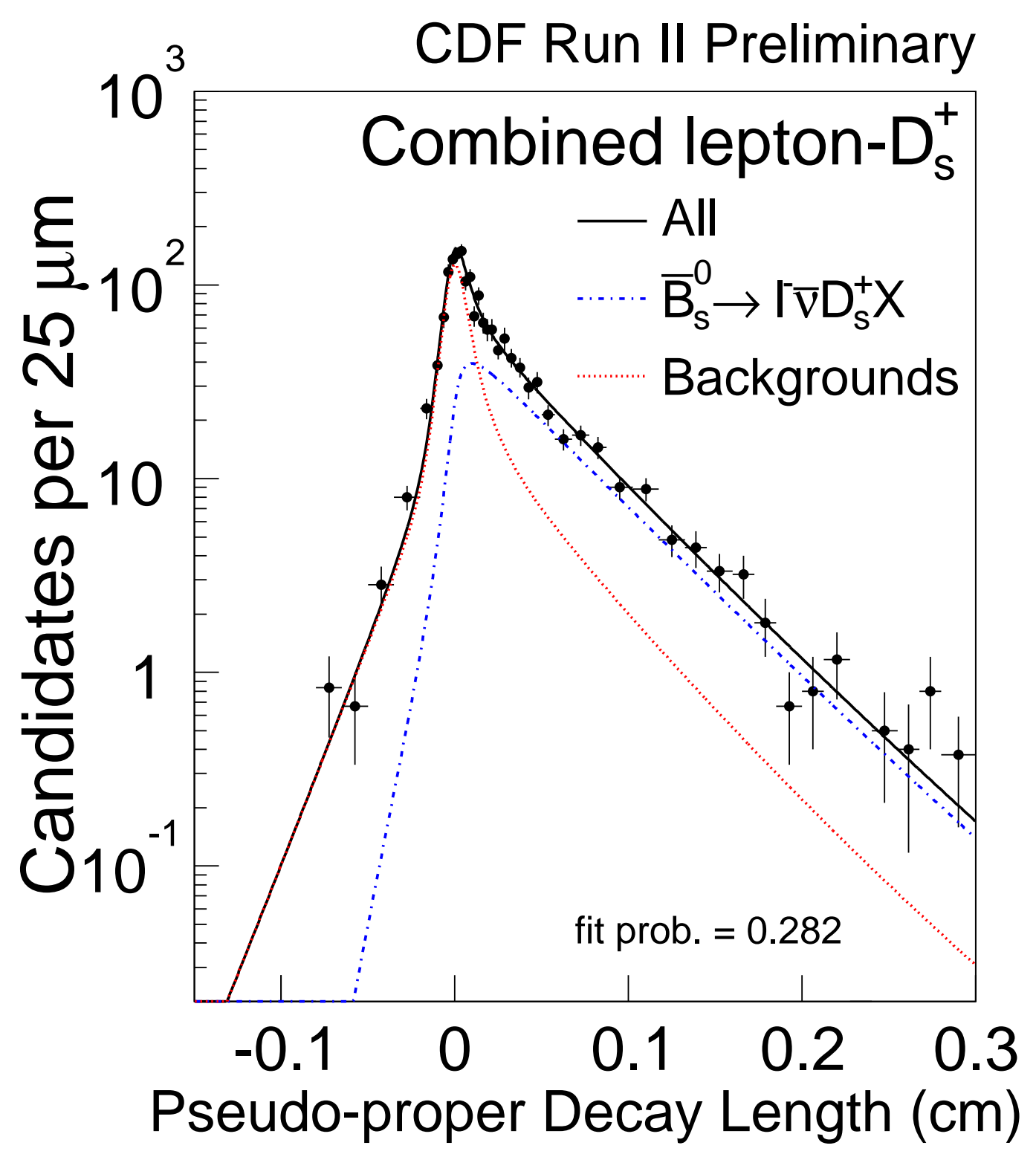

Figure 5.8: The lifetime fit result for the muon and electron combined dataset. Red curve shows the sum of all the backgrounds (combinatorial and physics backgrounds). 


\subsection{Systematic uncertainties}

In this Section we discuss various sources of systematic uncertainties in the $B_{s}^{0}$ meson lifetime measurement, and their effects on the final answer.

As described earlier, the only free parameter in the fit is the $B_{s}^{0}$ meson lifetime $c \tau\left(B_{s}^{0}\right)$, and all the others, such as the background fraction and its shape, are fixed to their pre-determined values. We estimate their effect as follows. We change the value of a parameter to another value, corresponding to a one-sigma uncertainty in the parameter, fix it at that value, and perform the lifetime fit. We interpret the observed change in the lifetime as the systematic uncertainty from this source (parameter). We repeat this procedure for all parameters, one by one, and add the individual uncertainties in quadrature to obtain the total systematic uncertainty.

\subsubsection{Physics background}

\section{Prompt charm background}

The main source of the systematics uncertainties lies in the treatment of the physics background, in particular the prompt charm background. Although we have measured their amount in the real data with a method that is mostly model-independent, it is subject to a statistical uncertainty arising from the finite statistics of the real data. Also, its shape is somewhat model-dependent, because we do not directly measure the origin of the "lepton", either prompt or from the other charm hadron, that is associated with the prompt $D_{s}^{+}$meson.

We estimate their effects on the $B_{s}^{0}$ meson lifetime in the way we described at the beginning of this Section. The lifetime numbers obtained under different amounts of the background is listed in Table 5.8.

\section{Bottom background}

As discussed previously, the main process of the bottom background is the decays of non-strange $B$ mesons to two charm hadrons,

$$
B^{+} / B^{0} \rightarrow D_{s}^{+} \bar{D} X
$$

The inclusive branching fraction of the non-strange $B$ mesons to a final state involving a $D_{s}^{+}$meson has been measured with reasonable precision:

$$
\mathcal{B}\left(\bar{B} \rightarrow D_{s}^{ \pm} X\right)=(10.5 \pm 2.6) \% .
$$

However, we are interested in their contribution relative to the $B_{s}^{0}$ meson signal, and there a considerable systematic uncertainty arises from the $b$-quark 
fragmentation fraction. In the Monte Carlo calculation of this background process, we used the fraction $f_{s} / f_{u}=0.3\left(f_{u}=f_{d}\right)$, which is compatible with what PDG quotes, $f_{s} / f_{u}=0.27 \pm 0.03[1]$. However, the PDG value assumes identical $b$-quark hadronization in $p \bar{p}$ collisions (Tevatron) and $Z^{0}$ decay (LEP), which may or may not be true.

We can measure, or at least check, this quantity by comparing the yields of the $D_{s}^{+}$meson to the $D^{+}$meson, because the former should come from the $\bar{B}_{s}^{0}$ meson decays while the latter from non-strange $\bar{B}$ mesons. We have already observed the $D^{+}$meson signal, in the same final state $\phi \pi^{+}$.

The mass distributions are shown in Figure 3.7. We count the numbers of $D_{s}^{+}$and $D^{+}$signals in the $M\left(K^{+} K^{-} \pi^{+}\right)$distribution, and we find ${ }^{2}$

$$
\begin{aligned}
\frac{N\left(D^{+}\right)}{N\left(D_{s}^{+}\right)} & =\frac{191.3 \pm 28.8}{800.6 \pm 40.9} \\
& =0.239 \pm 0.038
\end{aligned}
$$

The quantity is related with the fragmentation fractions and the branching fractions of various decay modes by

$$
\frac{N\left(D^{+}\right)}{N\left(D_{s}^{+}\right)}=\frac{2 f_{u}}{f_{s}} \frac{\mathcal{B}\left(\bar{B} \rightarrow \ell^{-} \bar{\nu} D^{+} X\right) \cdot \mathcal{B}\left(D^{+} \rightarrow \phi \pi^{+}\right) \cdot \mathcal{B}\left(\phi \rightarrow K^{+} K^{-}\right)}{\left.\mathcal{B}\left(\ell^{-}\right) \bar{\nu} D_{s}^{+} X\right) \cdot \mathcal{B}\left(D_{s}^{+} \rightarrow \phi \pi^{+}\right) \cdot \mathcal{B}\left(\phi \rightarrow K^{+} K^{-}\right)}
$$

Here we assumed $f_{u}=f_{d}$, and the efficiencies for $D^{+}$and $D_{s}^{+}$reconstruction are the same. We also use the following branching fractions from PDG [1] :

$$
\begin{aligned}
\mathcal{B}\left(\bar{B} \rightarrow \ell^{-} \bar{\nu} D^{+} X\right) & =(2.8 \pm 0.9) \times 10^{-2} \\
\mathcal{B}\left(\bar{B}_{s}^{0} \rightarrow \ell^{-} \bar{\nu} D_{s}^{+} X\right) & =(7.9 \pm 2.4) \times 10^{-2} \\
\mathcal{B}\left(D^{+} \rightarrow \phi \pi^{+}\right) \cdot \mathcal{B}\left(\phi \rightarrow K^{+} K^{-}\right) & =(3.1 \pm 0.3) \times 10^{-3} \\
\mathcal{B}\left(D_{s}^{+} \rightarrow \phi \pi^{+}\right) & =(3.6 \pm 0.9) \times 10^{-2} \\
\mathcal{B}\left(\phi \rightarrow K^{+} K^{-}\right) & =(49.1 \pm 0.6) \times 10^{-2}
\end{aligned}
$$

From the branching fractions and the measured ratio of $N\left(D^{+}\right) / N\left(D_{s}^{+}\right)$, we find

$$
f_{s} / f_{u}=0.52 \pm 0.28 \text {. }
$$

The result is consistent with the number used in the Monte Carlo generator $\left(f_{s} / f_{u}=0.3\right)$. A larger $f_{s}$ value means a smaller background from non-strange $B$ meson decays. But to be conservative, We consider the range $0.2<f_{s} / f_{u}<$

\footnotetext{
${ }^{2}$ These are the numbers from the muon sample. We should have used the numbers for the combined electron and muon sample, $285.1 \pm 34.9$ and $1154.4 \pm 49.0$, which fortunately make little difference.
} 
0.52 to estimate the bottom background fraction $f_{b}$. The corresponding values of $f_{b}$ are:

$$
f_{b}= \begin{cases}9.2 \sim 3.7 \% & \text { for } \mu^{-} D_{s}^{+} \text {sample } \\ 4.9 \sim 2.0 \% & \text { for } e^{-} D_{s}^{+} \text {sample }\end{cases}
$$

With these numbers the lifetime fits are performed again, and we assign the observed change as a systematic uncertainty.

To estimate the uncertainty due to the shape of the bottom background, we consider the differences among the three subprocesses that we considered. We change the shape $\mathcal{F}_{b}$ to that of only the $B^{-} / \bar{B}^{0} \rightarrow \bar{D} D_{s}^{+}$process, or to that of the $B_{s} \rightarrow \tau^{-} \bar{\nu} D_{s}^{+} X$ process. They are shown with the colored histograms in Figure 5.6.

We assign the observed changes in the lifetime as the systematic uncertainty from the physics background treatment.

\subsubsection{Missing momentum correction}

The missing momentum correction, namely the $K$-factor distribution, is also subject to some uncertainty. We consider four factors affecting the $K$-factor distribution. They are the $p_{T}(B)$ spectrum, the $B$ meson decay model, the electron identification cuts, and the $D_{s}^{* *+}$ fraction in semileptonic decays of the $\bar{B}_{s}^{0}$ meson.

The $B$ meson transverse momentum spectrum is closely related with the momenta of the decay particles. Since we are triggering on a lepton at a fixed threshold, and the $B$ meson spectrum is falling steeply as a function of $p_{T}$, the events surviving the kinematic cuts tend to come from $B$ mesons with smaller $p_{T}$ than with very large $p_{T}$. As mentioned in Section 4.1.1, we have modified the original $p_{T}(B)$ spectrum in the Monte Carlo generator with a correction factor $p_{T}(B)^{\alpha}$, where the parameter $\alpha$ is measured to be -0.732 to match the inclusive $B$ hadron spectrum measured in the Run-II $B \rightarrow J / \psi X$ events. To estimate systematic effects, we consider the values of $\alpha$ of 0.0 and -1.4640 .

The second item, the $B$ meson decay model, affects the decay kinematics and thus the momentum ratio $K$. The EvtGen generator uses the ISGW2 model for the semileptonic decays of the $B$ mesons. We change the $B$ decay generator to QQ[58], which uses the ISGW model, and calculate the $K$ factor distributions. The two sets of distributions are shown in Figure 5.9. The lifetime fits are repeated with the $K$ factors estimated using QQ.

The next item concerns the effect of electron identification cuts. As shown in the Figure 5.1, there is a small difference in the distributions of the momentum ratio $K$ between the muon and electron samples. Since the electron identification cuts prefer events with a large opening angle between the lepton and the other decay products of the $B$ meson, it results in a harder distribution 
of $K$. On the other hand, the muon $K$ factor distribution is pretty insensitive. We find an almost identical $K$ distribution if we use the parametric simulation. To account for possible incompleteness in simulating electron cuts, we assign a systematic uncertainty that corresponds to a shift in the lifetime when we replace the $K$ distribution for the electron sample with that for the muon sample.

The last item related to the momentum correction is the fraction of the $D_{s}^{* *+}$ mesons produced in the semileptonic decays of the $\bar{B}_{s}^{0}$ meson. As can be seen in Figure 5.10, the $K$ distribution is softer for the decay $\bar{B}_{s}^{0} \rightarrow \ell^{-} \bar{\nu} D_{s}^{* *+}$. It is because of the larger $D^{* *}$ meson masses, and of the additional pion(s) that is present in the final states. We change $f^{* *}$ in the range $0.36 \pm 0.12$, the same range considered for the non-strange $B$ mesons. and calculate the $K$ factor distributions with the new $f^{* *}$, and repeat the lifetime fit.

The changes in the lifetime value from these sources are assigned as the systematic uncertainties.

\subsubsection{Other sources of systematic uncertainties}

The amount of the $D_{s}^{+}$meson signal is subject to a statistical uncertainty. We change $f_{\text {sig }}$ from the central value of the measurement to the values corresponding to $\pm 1 \sigma$.

For the vertex determination resolution, we change the scale factor $s$ to 1.3 and to 1.8, for the muon and electron datasets simultaneously. With the new value the lifetime fits are repeated. We assign the observed changes in the lifetime as a systematic uncertainty.

We have applied a loose cut on the $D_{s}^{+}$decay length, $L_{x y}>0$. To evaluate the effect of the cut, we use a signal Monte Carlo sample with full detector simulation. We fit the sample with and without the $L_{x y}>0$ cut. The difference in the fit results is interpreted as the systematic uncertainty.

The presence of combinatorial background events under the $D_{s}^{+}$meson mass peaks are taken into account by including the term in the likelihood function for the background component. Its shape is estimated using events on the mass sideband, thus reducing model dependence. However, they are determined with finite event statistics, and thus are subject to statistical uncertainty. Also, the functional shape we used to describe the $c t^{*}$ distribution of the combinatorial background may not be fully adequate. Finally, the sideband events may not fully represent the events under the mass peak. We estimate these effects by performing the lifetime fit using the signal and sideband samples simultaneously, with common combinatorial background parameters. The lifetime value changes very little, only $0.2 \mu \mathrm{m}$, but the statistical uncertainty increases from $\pm 16.6 \mu \mathrm{m}$ to $\pm 17.4 \mu \mathrm{m}$. The difference in quadrature, $5.2 \mu \mathrm{m}$, reflects the 
statistical uncertainty in the background shape. Therefore, we add this as a systematic uncertainty.

Finally, we assign a systematic uncertainty of $2 \mu \mathrm{m}$ due to possible residual misalignment of the silicon detector.

The uncertainties from all sources are added in quadrature, and are listed in Table 5.9.

We note that some of the quantities contributing to systematic uncertainties are measured or determined using real data, and their limited statistical precision are the source of uncertainties. Therefore, they are expected to be reduced as the signal statistics increase. 


\begin{tabular}{|c|c|c|c|c|}
\hline $\begin{array}{l}\text { Source and } \\
\text { Parameter }\end{array}$ & $\begin{array}{c}\text { Default } \\
\text { value }\end{array}$ & $\begin{array}{c}\text { Changed } \\
\text { to }\end{array}$ & $\begin{array}{c}c \tau\left(\bar{B}_{s}^{0}\right) \\
(\mu \mathrm{m})\end{array}$ & $\begin{array}{l}\text { Shift } \\
(\mu \mathrm{m})\end{array}$ \\
\hline No change & & & $414.0 \pm 16.6$ & \\
\hline \multirow[t]{2}{*}{$f_{c}$ for $\mu$} & $3.6 \%$ & $0 \%$ & $407.2 \pm 16.2$ & -6.8 \\
\hline & & $8.1 \%$ & $422.4 \pm 17.1$ & +8.4 \\
\hline$f_{c}$ for $e$ & $0 \%$ & $7.1 \%$ & $420.2 \pm 16.9$ & +6.2 \\
\hline \multirow{2}{*}{$\mathcal{F}_{c}$ for $\mu, e$} & $\mathcal{F}_{c}^{\text {zero }}$ & $\mathcal{F}_{c}^{\text {non-zero }}, c \bar{c} \rightarrow \ell^{-} D_{s}^{+}$ & $412.5 \pm 16.6$ & -1.5 \\
\hline & & $\mathcal{F}_{c}^{\text {non-zero }}, c \bar{c} \rightarrow h^{-} D_{s}^{+}$ & $413.2 \pm 16.6$ & -0.8 \\
\hline \multirow[t]{2}{*}{$f_{b}$ for $\mu, e$} & $6.3 \%(\mu), 3.4 \%(e)$ & $3.7 \%(\mu), 2.0 \%(e)$ & $417.1 \pm 16.2$ & +3.1 \\
\hline & & $9.2 \%(\mu), 4.9 \%(e)$ & $410.6 \pm 17.0$ & -3.6 \\
\hline \multirow[t]{2}{*}{$\mathcal{F}_{b}$ for $\mu, e$} & Total $b$ BG & $B \rightarrow \bar{D} D_{s}^{+}$only & $411.1 \pm 16.6$ & -2.9 \\
\hline & & $\bar{B}_{s}^{0} \rightarrow \tau \bar{\nu} D_{s}^{+}$only & $408.9 \pm 16.6$ & -5.1 \\
\hline \multirow[t]{2}{*}{$\alpha$} & -0.732 & 0.0 & $409.1 \pm 16.4$ & -4.9 \\
\hline & & -1.464 & $418.6 \pm 16.7$ & +4.6 \\
\hline MC generator & EvtGen & QQ & $419.0 \pm 16.7$ & +5.0 \\
\hline$K$ factor for $e$ & electron $\mathrm{MC}$ & muon $\mathrm{MC}$ & $412.9 \pm 16.5$ & -1.1 \\
\hline \multirow[t]{2}{*}{$f^{* *}$} & 0.36 & 0.24 & $415.5 \pm 16.6$ & +1.5 \\
\hline & & 0.48 & $412.2 \pm 16.5$ & -1.8 \\
\hline \multirow[t]{2}{*}{$f_{\text {sig }}$ for $\mu$} & 0.503 & $-1 \sigma$ & $417.6 \pm 16.9$ & +3.6 \\
\hline & & $+1 \sigma$ & $410.6 \pm 16.3$ & -3.4 \\
\hline \multirow[t]{2}{*}{$f_{\text {sig }}$ for $e$} & 0.503 & $-1 \sigma$ & $417.0 \pm 16.8$ & +3.0 \\
\hline & & $+1 \sigma$ & $411.1 \pm 16.4$ & -2.9 \\
\hline \multirow[t]{2}{*}{ Scale factor $s(\mu, e)$} & $1.59(\mu), 1.56(e)$ & 1.3 & $418.0 \pm 16.7$ & +4.0 \\
\hline & & 1.8 & $411.1 \pm 16.5$ & -2.9 \\
\hline Comb. BG par. & fix & simultaneous fit & $(414.2) \pm 17.4$ & \\
\hline
\end{tabular}

Table 5.8: Summary of fit results performed for evaluation of systematic uncertainties. Symbols $\mu$ and $e$ denote the muon and electron data sets. If they both appear in a single row, the parameter is considered correlated between the two data sets and is changed simultaneously for the two data sets. Otherwise it is considered independent. 


\begin{tabular}{lc}
\hline \hline Source & $\begin{array}{c}\text { Contribution to } \\
c \tau\left(\bar{B}_{s}^{0}\right)(\mu \mathrm{m})\end{array}$ \\
\hline Prompt charm background & \\
Prompt charm fraction $\left(f_{c}\right)$ & ${ }_{-6.8}^{+0.4}$ \\
Prompt charm shape $\left(\mathcal{F}_{c}\right)$ & ${ }_{-1.5}^{+0.0}$ \\
Bottom background & \\
Bottom background fraction $\left(f_{b}\right)$ & \pm 3.6 \\
Bottom background shape $\left(\mathcal{F}_{b}\right)$ & ${ }_{-5.1}^{+0.0}$ \\
Missing momentum correction & \\
$p_{T}(B)$ spectrum & \pm 4.9 \\
$B$ decay model & ${ }_{-0.0}^{+5.0}$ \\
Electron cuts & ${ }_{-1.1}^{+0.0}$ \\
$D_{s}^{* *+}$ fraction $\left(f^{* *}\right)$ & \pm 1.8 \\
Signal fraction $\left(f_{\text {sig }}\right)$ & \pm 4.7 \\
Resolution scale factor & \pm 4.0 \\
Decay length cut & ${ }_{-2.3}^{+0.0}$ \\
Combinatorial background shape & \pm 5.2 \\
Detector alignment & \pm 2.0 \\
\hline Total & ${ }_{-13.8}^{+15.6}$ \\
\hline \hline
\end{tabular}

Table 5.9: Summary of systematic uncertainties in the $\bar{B}_{s}^{0}$ meson lifetime measurement. 

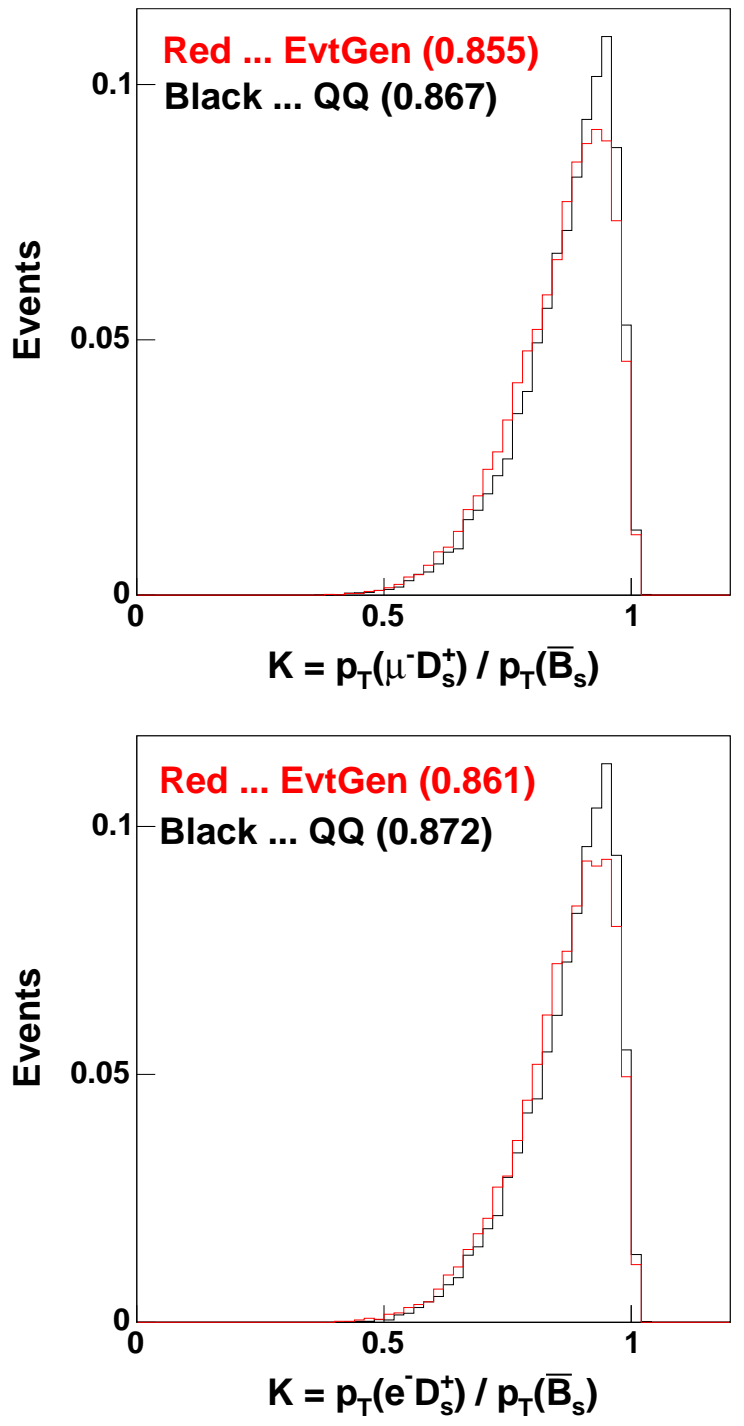

Figure 5.9: Comparison of the $K$ factor distributions with different generators (EvtGen and QQ), for the muon and electron datasets. 

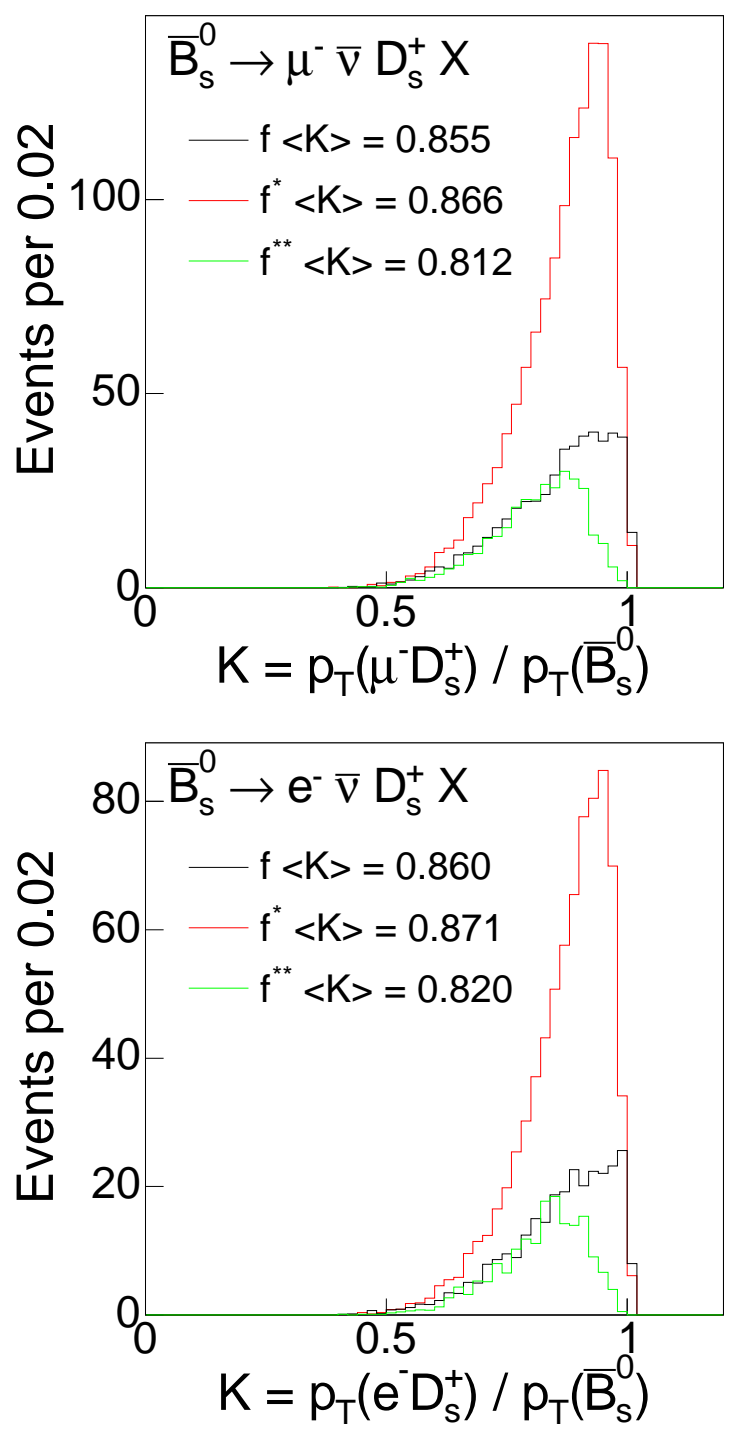

Figure 5.10: Comparison of the distributions of the momentum ratio $K$ for different semileptonic decay modes, $\bar{B}_{s}^{0} \rightarrow \ell^{-} \bar{\nu} D_{s}^{+}, \rightarrow \ell^{-} \bar{\nu} D_{s}^{*+}$ and $\rightarrow \ell^{-} \bar{\nu} D_{s}^{* *+}$. 


\subsection{Ratio of the $\bar{B}_{s}^{0}$ and $\bar{B}^{0}$ meson lifetimes}

To compare the measured lifetime with the HQE prediction, it is best to take the ratio of the $\bar{B}_{s}^{0}$ and $\bar{B}^{0}$ meson lifetimes. Here we do so by comparing the lifetimes of $\bar{B}_{s}^{0}$ and $\bar{B}^{0}$ meson measured in this analysis:

$$
\begin{aligned}
& c \tau\left(\bar{B}_{s}^{0}\right)=414.0 \pm 16.6 \mu \mathrm{m} \\
& c \tau\left(\bar{B}^{0}\right)=441.5 \pm 10.9 \mu \mathrm{m}
\end{aligned}
$$

The quoted uncertainties are statistical only. From these numbers we find the ratio of the lifetimes to be

$$
\tau\left(\bar{B}_{s}^{0}\right) / \tau\left(\bar{B}^{0}\right)=0.938 \pm 0.044 .
$$

Since some of the systematic uncertainties are common to the two measurements and are correlated, we need to re-evaluate them again. Sometimes we need to move the parameters in the same directions together. They are given in Table 5.10.

Although most of the systematics uncertainties are already explained in the Section 4.8 and 5.6, we add some words on a systematic effect from the $p_{T}(B)$ spectrum. At the Monte Carlo generation we have used identical $p_{T}(B)$ spectra for the $B^{-} / \bar{B}^{0}$ and $\bar{B}_{s}^{0}$ mesons. However the $p_{T}$ spectrum of the $\bar{B}_{s}^{0}$ meson is not well measured. Therefore, we examine the following two extreme cases for the $p_{T}$ spectra of the $B$ mesons.

- $\alpha=0.0$ for $B^{-} / \bar{B}^{0}, \quad \alpha=-1.464$ for $\bar{B}_{s}^{0}$

- $\alpha=-1.464$ for $B^{-} / \bar{B}^{0}, \quad \alpha=0.0$ for $\bar{B}_{s}^{0}$

The uncertainties from all sources are added in quadrature. The total systematic uncertainty in the ratio $\tau\left(\bar{B}_{s}^{0}\right) / \tau\left(\bar{B}^{0}\right)$ amounts to about \pm 0.05 . 


\begin{tabular}{lc}
\hline \hline Source & $\begin{array}{c}\text { Contribution to } \\
\tau\left(\bar{B}_{s}^{0}\right) / \tau\left(\bar{B}^{0}\right)\end{array}$ \\
\hline Prompt charm background & \\
Prompt charm fraction $\left(f_{c}\right)$ & ${ }_{-0.020}^{+0.025}$ \\
Prompt charm shape $\left(\mathcal{F}_{c}\right)$ & ${ }_{-0.012}^{+0.011}$ \\
Bottom background & ${ }_{-0.008}^{+0.009}$ \\
Bottom background fraction $\left(f_{b}\right)$ & ${ }_{-0.012}^{+0.003}$ \\
Bottom background shape $\left(\mathcal{F}_{b}\right)$ & \\
Sample composition $\left(\right.$ affects only $\left.\tau\left(\bar{B}^{0}\right)\right)$ & ${ }_{-0.023}^{+0.018}$ \\
$D^{* *}$ composition $\left(P_{V}\right)$ & ${ }_{-0.002}^{+0.000}$ \\
$\pi_{s}^{+}$reconstruction & \\
Missing momentum correction & ${ }_{-0.016}^{+0.022}$ \\
$p_{T}(B)$ spectrum & - \\
Electron cuts & \pm 0.012 \\
$B$ decay model & ${ }_{-0.011}^{+0.017}$ \\
$D_{s}^{* *+}$ fraction $\left(f^{* *}\right)$ & \pm 0.011 \\
Signal fraction $\left(f_{\text {sig }}\right)$ & \pm 0.004 \\
Resolution scale factor & - \\
Decay length cut & \pm 0.012 \\
Combinatorial background shape & - \\
Detector alignment & ${ }_{-0.046}^{+0.049}$ \\
\hline Total & \\
\hline \hline
\end{tabular}

Table 5.10: Summary of systematic uncertainties in the $\bar{B}_{s}^{0} / \bar{B}^{0}$ lifetime ratio. 


\subsection{A non-zero $\Delta \Gamma_{s} / \Gamma_{s}$ value}

In the $B_{s}^{0} \bar{B}_{s}^{0}$ system, the two flavor eigenstates are expected to mix with each other and result in the new states $B_{s S}^{0}$ and $B_{s L}^{0}$, which should have different masses and lifetimes. In particular, the width difference is predicted to be sizable, of order $10 \%$ in $\Delta \Gamma_{s} / \Gamma_{s}$. Also, there is an experimental indication that the width difference is indeed large and detectable, such as the CDF analysis of $B_{s}^{0} \rightarrow J / \psi \phi$ decays [69].

In flavor specific final states, such as the semileptonic decay, the timedependence of the decay probability is written as a sum of the two lifetime components $[70]$ :

$$
P(t)=\frac{1}{\tau_{L}+\tau_{S}}\left[e^{-t / \tau_{L}}+e^{-t / \tau_{S}}\right],
$$

where $\tau_{S}$ and $\tau_{L}$ are the lifetimes of the short-lived $\left(B_{s S}^{0}\right)$ and long-lived $\left(B_{s L}^{0}\right)$ meson states. The mean value of the probability density function $P(t)$ above becomes

$$
\begin{aligned}
\langle\tau\rangle & \equiv \int_{0}^{\infty} t P(t) d t \\
& =\frac{1}{\tau_{L}+\tau_{S}}\left(\tau_{L}^{2}+\tau_{S}^{2}\right) \\
& =\frac{1}{\Gamma_{s}} \frac{\Gamma_{s}^{2}+\left(\Delta \Gamma_{s} / 2\right)^{2}}{\Gamma_{s}^{2}-\left(\Delta \Gamma_{s} / 2\right)^{2}},
\end{aligned}
$$

where $\Gamma_{s} \equiv\left(\Gamma_{s S}+\Gamma_{s L}\right) / 2$ is the average decay width, $\Delta \Gamma_{s} \equiv \Gamma_{s S}-\Gamma_{s L}$ is the width difference, and $\Gamma_{s S, L} \equiv \Gamma_{s} \pm \Delta \Gamma_{s} / 2=1 / \tau_{S, L}$. Note that

$$
\begin{aligned}
\langle\tau\rangle & >1 / \Gamma_{s} \\
& \neq 1 / \Gamma_{s} .
\end{aligned}
$$

In this analysis we have thus far assumed that the width difference is negligible, and used a single exponential function for the lifetime fits. If we fit the distributions with single exponential fits, but if $\Delta \Gamma_{s}$ is not negligible, we may expect to obtain [71]

$$
\tau_{\text {single }} \simeq\langle\tau\rangle
$$

where $\langle\tau\rangle$ is defined above.

Alternatively, we could fit for the average width $\Gamma_{s}$ by assuming a value of $\Delta \Gamma_{s} / \Gamma_{s}$. We consider $\Delta \Gamma_{s} / \Gamma_{s}=0.37_{-0.20}^{+0.17}$, which is quoted in the 2005 web update version of PDG. For $\Delta \Gamma_{s} / \Gamma_{s}=0.37$, we find

$$
c / \Gamma_{s}=386.7 \pm 16.0 \mu \mathrm{m},
$$

where the uncertainty is statistical only. From this value and $\Delta \Gamma_{s} / \Gamma_{s}$, we calculate $\tau_{L}$ and $\tau_{S}$. Table 5.11 summarizes these numbers, including other values of $\Delta \Gamma_{s} / \Gamma_{s}$ we have assumed. 


\begin{tabular}{cccc}
\hline \hline$\Delta \Gamma_{s} / \Gamma_{s}$ & $c \tau_{S}(\mu \mathrm{m})$ & $c \tau_{L}(\mu \mathrm{m})$ & $c / \Gamma_{s}(\mu \mathrm{m})$ \\
\hline 0.17 & $376.1 \pm 15.2$ & $446.0 \pm 18.0$ & $408.1 \pm 16.5$ \\
0.37 & $326.3 \pm 13.5$ & $474.4 \pm 19.6$ & $386.7 \pm 16.0$ \\
0.54 & $281.7 \pm 11.9$ & $490.1 \pm 20.7$ & $357.8 \pm 15.1$ \\
\hline \hline
\end{tabular}

Table 5.11: Results of the two exponential fits with fixed $\Delta \Gamma_{s} / \Gamma_{s}$ values $\left(\Delta \Gamma_{s}=\right.$ $\left.0.37_{-0.20}^{+0.17}[1]\right)$. Quoted uncertainties are statistical only.

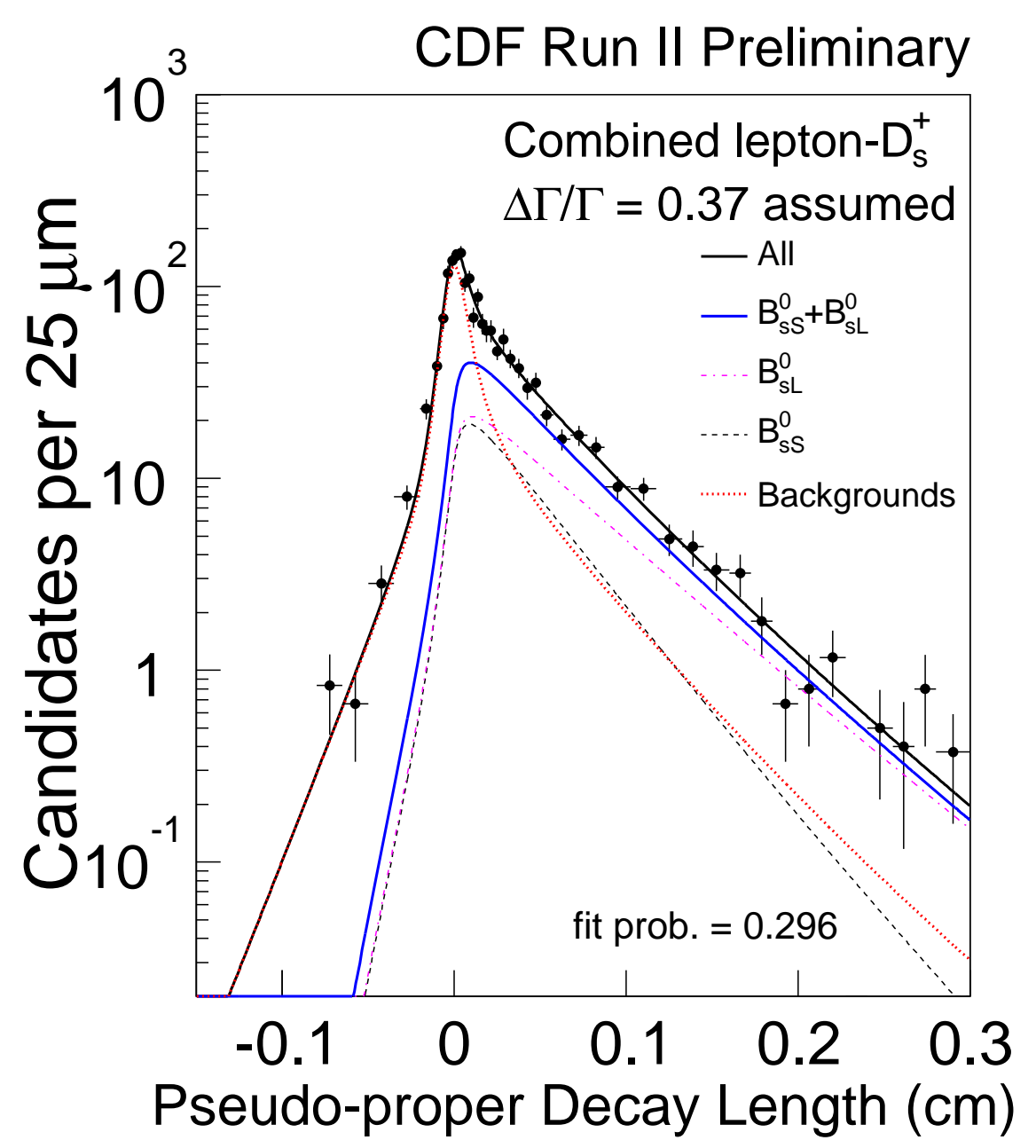

Figure 5.11: Result of the two exponential fits with $\Delta \Gamma_{s} / \Gamma_{s}=0.37$. 



\section{Chapter 6}

\section{Results and conclusions}

We have measured the lifetimes of the $B^{-}, \bar{B}^{0}$ and $\bar{B}_{s}^{0}$ mesons using the partially reconstructed semileptonic decays. The lifetimes are measured to be

$$
\begin{aligned}
c \tau\left(B^{-}\right) & =495.6 \pm 8.6_{-9.4}^{+10.0} \mu \mathrm{m} \\
c \tau\left(\bar{B}^{0}\right) & =441.5 \pm 10.9 \pm 16.3 \mu \mathrm{m} \\
c \tau\left(\bar{B}_{s}^{0}\right) & =414.0 \pm 16.6_{-13.8}^{+15.6} \mu \mathrm{m}
\end{aligned}
$$

or

$$
\begin{aligned}
\tau\left(B^{-}\right) & =1.653 \pm 0.029{ }_{-0.031}^{+0.033} \mathrm{ps} \\
\tau\left(\bar{B}^{0}\right) & =1.473 \pm 0.036 \pm 0.054 \mathrm{ps} \\
\tau\left(\bar{B}_{s}^{0}\right) & =1.381 \pm 0.055_{-0.046}^{+0.052} \mathrm{ps}
\end{aligned}
$$

And we find the ratio of the lifetimes to be

$$
\begin{aligned}
\tau\left(B^{-}\right) / \tau\left(\bar{B}^{0}\right) & =1.123 \pm 0.040_{-0.039}^{+0.041} \\
\tau\left(\bar{B}_{s}^{0}\right) / \tau\left(\bar{B}^{0}\right) & =0.938 \pm 0.044_{-0.046}^{+0.049}
\end{aligned}
$$

where the first and second uncertainties are statistical and systematic, respectively.

These results are consistent with the current world averages [1][39]. The ratio of $\tau\left(B^{-}\right)$and $\tau\left(\bar{B}^{0}\right)$ is slightly deviated from one, and the ratio of $\tau\left(\bar{B}_{s}^{0}\right)$ and $\tau\left(\bar{B}^{0}\right)$ is consistent with unity, which are consistent with a theoretical prediction [6]. Figure 6.1-6.5 show a comparison of results from other experiments [39] and our results.

If we assume a non-zero value of $\Delta \Gamma_{s} / \Gamma_{s}$, we get the following results :

$$
\begin{aligned}
\Delta \Gamma_{s} / \Gamma_{s} & =0.37 \text { (assumed) } \\
1 / \Gamma_{s} & =1.290 \pm 0.053 \mathrm{ps},
\end{aligned}
$$


which correspond to

$$
\begin{aligned}
& 1 / \Gamma_{s S}=1.088 \pm 0.045 \mathrm{ps} \\
& 1 / \Gamma_{s L}=1.582 \pm 0.654 \mathrm{ps}
\end{aligned}
$$

where the uncertainties are statistical only. 


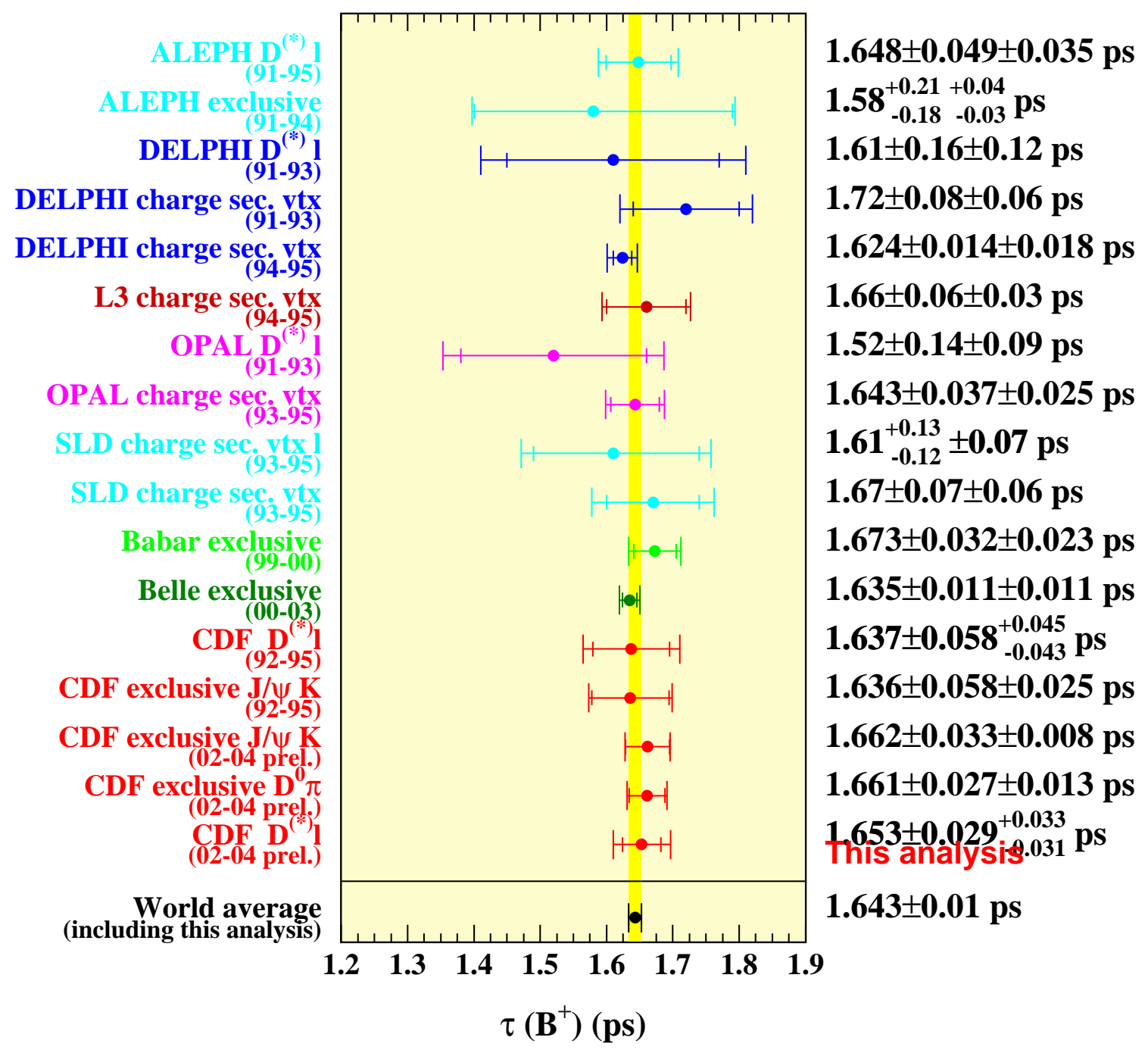

Figure 6.1: A comparison of $\tau\left(B^{+}\right)$measured by other experiments and this analysis. The result of this analysis is included in the world average. 


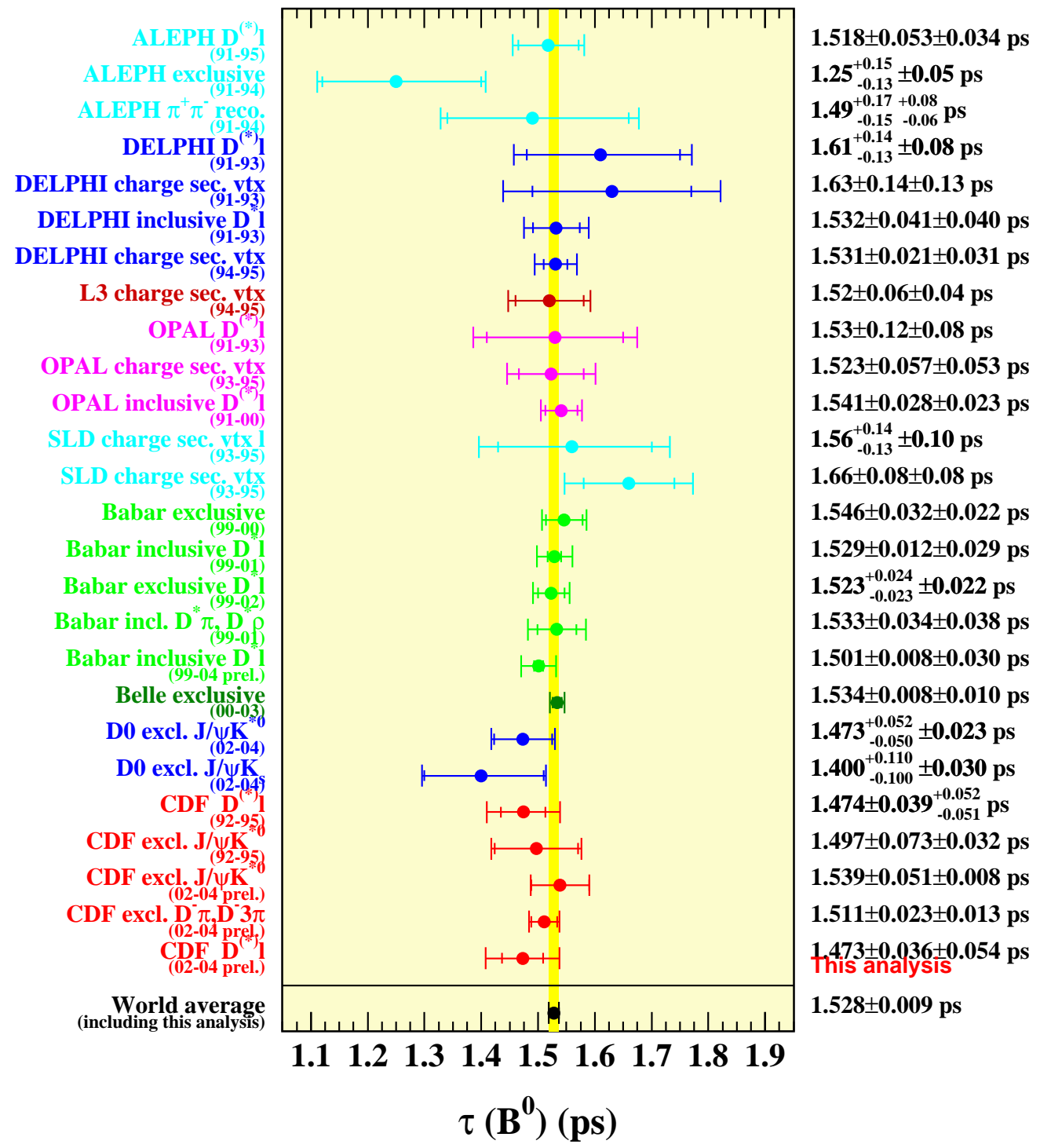

Figure 6.2: A comparison of $\tau\left(B^{0}\right)$ measured by other experiments and this analysis. The result of this analysis is included in the world average. 


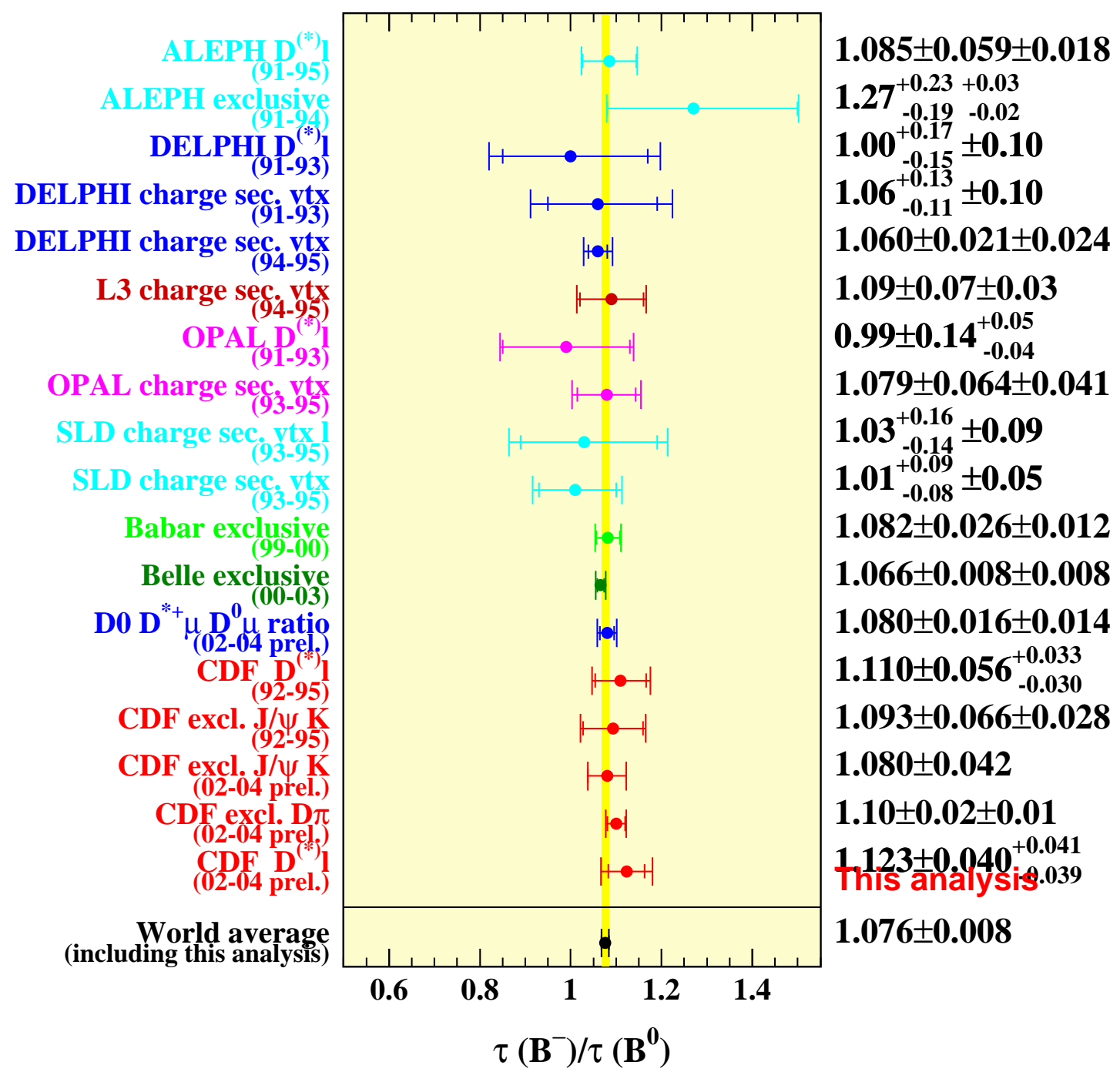

Figure 6.3: A comparison of $B^{0} / B^{+}$lifetime ratio with other measurements and this analysis. The result of this analysis is included in the world average. 


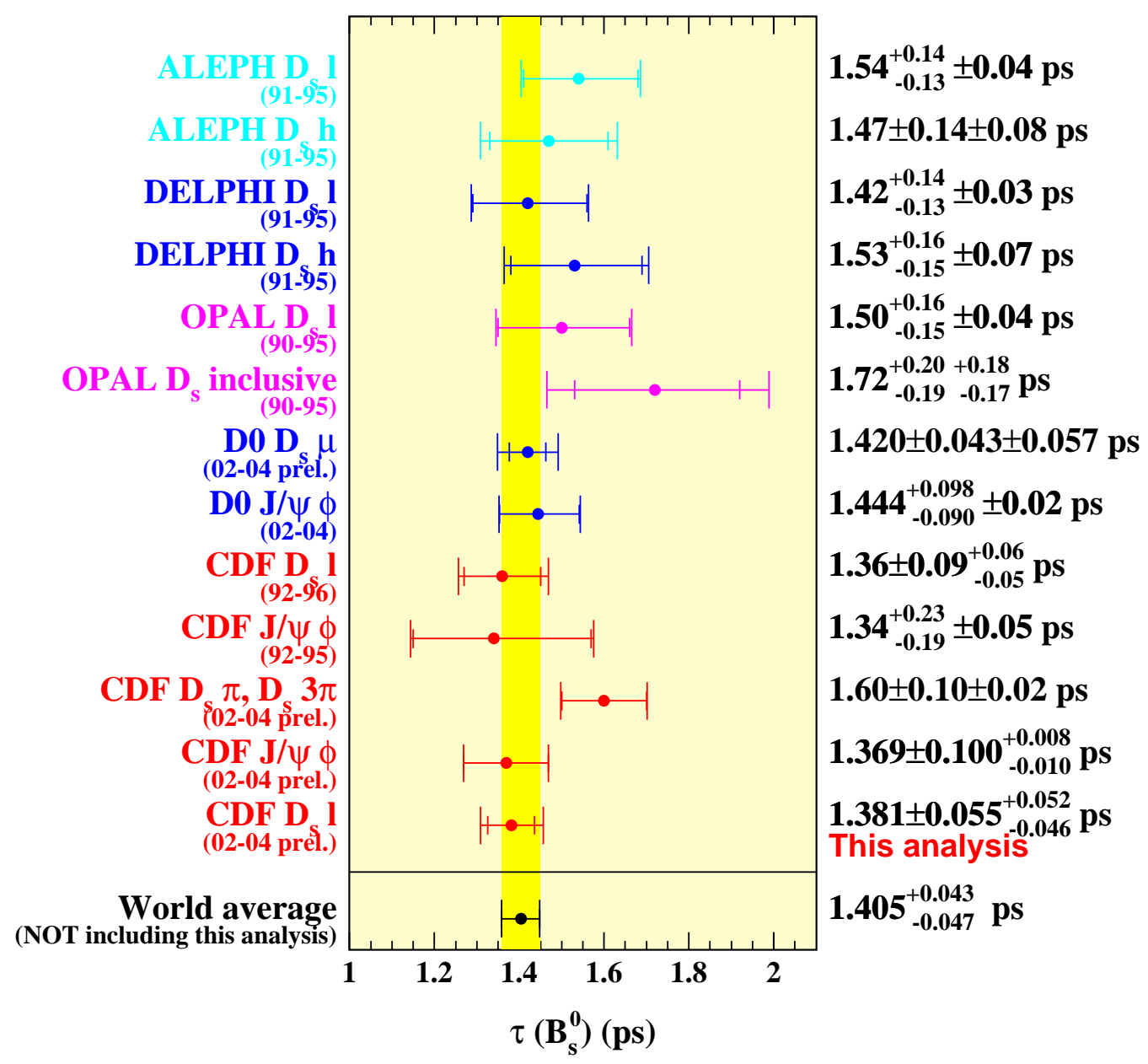

Figure 6.4: A comparison of $\tau\left(B_{s}^{0}\right)$ measured by other experiments and this analysis. The result of this analysis is not included in the world average. 


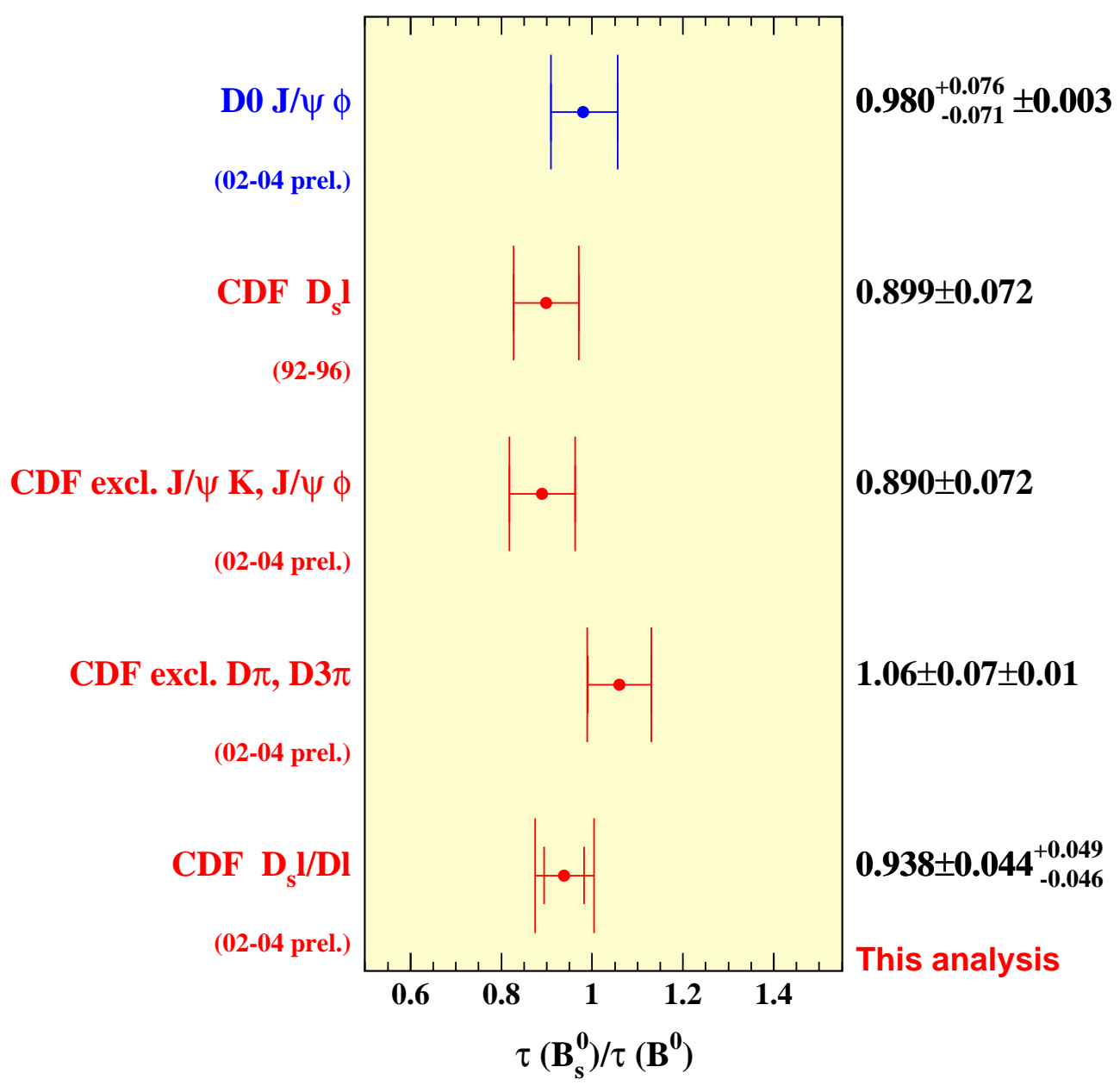

Figure 6.5: Measurements of the ratio of the $\bar{B}_{s}^{0}$ and $\bar{B}^{0}$ meson lifetimes from other experiments and this analysis. Only those measurements which quote the ratio explicitly are listed. 



\section{Appendix A}

\section{Probability density function formulae}

\section{A.1 Signal probability density function}

$$
\begin{aligned}
& \mathcal{F}_{B}\left(x, \sigma_{x} ; c \tau, s\right) \\
= & \int_{0}^{\infty} d K H(K) \int_{-\infty}^{\infty} d y \theta(x-y) \frac{K}{c \tau} \exp \left\{-\frac{K}{c \tau}(x-y)\right\} \frac{1}{\sqrt{2 \pi} s \sigma_{x}} \exp \left(-\frac{y^{2}}{2 s^{2} \sigma_{x}^{2}}\right) \\
= & \int_{0}^{\infty} d K H(K) \int_{-\infty}^{x} d y \frac{K}{c \tau} \exp \left\{-\frac{K}{c \tau}(x-y)\right\} \frac{1}{\sqrt{2 \pi} s \sigma_{x}} \exp \left(-\frac{y^{2}}{2 s^{2} \sigma_{x}^{2}}\right) \\
= & \int_{0}^{\infty} d K H(K) \frac{K}{c \tau} \exp \left(-\frac{K x}{c \tau}+\frac{K^{2} s^{2} \sigma_{x}^{2}}{2 c^{2} \tau^{2}}\right) \frac{1}{\sqrt{2 \pi} s \sigma_{x}} \int_{-\infty}^{x} d y \exp \left\{-\frac{1}{2}\left(\frac{y}{s \sigma_{x}}-\frac{K s \sigma_{x}}{c \tau}\right)^{2}\right\} \\
= & \int_{0}^{\infty} d K H(K) \frac{K}{c \tau} \exp \left(-\frac{K x}{c \tau}+\frac{K^{2} s^{2} \sigma_{x}^{2}}{2 c^{2} \tau^{2}}\right) \text { Freq }\left(\frac{x}{s \sigma_{x}}-\frac{K s \sigma_{x}}{c \tau}\right)
\end{aligned}
$$

where $\theta(x)$ is a step function,

$$
\theta(x)= \begin{cases}1 & \text { for } x \geq 0 \\ 0 & \text { for } x<0\end{cases}
$$

and Freq( $\mathrm{z})$ is an error function defined as follows.

$$
\operatorname{Freq}(z)=\frac{1}{\sqrt{2 \pi}} \int_{-\infty}^{z} d t e^{-\frac{t^{2}}{2}}
$$

Since the $K$ factor distribution $H(K)$ is given as a normalized histogram, the integral over $K$ is replaced with a finite sum.

$$
\begin{aligned}
\int_{0}^{\infty} d K H(K) & \rightarrow \sum_{i} H_{i} w_{i} \\
K & \rightarrow K_{i}
\end{aligned}
$$


where $H_{i}, w_{i}$ and $K_{i}$ denote the content, bin width, and the value of $K$ in $i$ bin in the $K$ factor histogram. The sum is taken over all the bins in the $K$ factor histogram. Then $\mathcal{F}_{B}$ is expressed to be

$$
\mathcal{F}_{B}\left(x, \sigma_{x} ; c \tau, s\right)=\sum_{i} H_{i} w_{i} \frac{K_{i}}{c \tau} \exp \left(-\frac{K_{i} x}{c \tau}+\frac{K_{i}^{2} s^{2} \sigma_{x}^{2}}{2 c^{2} \tau^{2}}\right) \text { Freq }\left(\frac{x}{s \sigma_{x}}-\frac{K_{i} s \sigma_{x}}{c \tau}\right)
$$

\section{A.2 Combinatorial background modeling func- tion}

$$
\begin{aligned}
& F_{\mathrm{BG}}\left(x ; \sigma, f_{1+}, \lambda_{1+}, f_{2+}, \lambda_{2+}, f_{1-}, \lambda_{1-}, f_{2-}, \lambda_{2-}\right) \\
= & \left(1-f_{1+}-f_{2+}-f_{1-}-f_{2-}\right) \frac{1}{\sqrt{2 \pi} \sigma} \exp \left\{-\frac{x^{2}}{2 \sigma^{2}}\right\} \\
& +f_{1+} T\left(x ; \lambda_{1+}, \sigma\right)+f_{2+} T\left(x ; \lambda_{2+}, \sigma\right)+f_{1-} T\left(-x ; \lambda_{1-}, \sigma\right)+f_{2-} T\left(-x ; \lambda_{2-}, \sigma\right)
\end{aligned}
$$

where $T(x)$ is a normalized exponential smeared by Gaussian,

$$
\begin{aligned}
T(x ; \lambda, \sigma) & =\int_{-\infty}^{\infty} d y \theta(x-y) \frac{1}{\lambda} \exp \left\{-\frac{1}{\lambda}(x-y)\right\} \frac{1}{\sqrt{2 \pi} \sigma} \exp \left(-\frac{y^{2}}{2 \sigma^{2}}\right) \\
& =\frac{1}{\lambda} \exp \left(-\frac{x}{\lambda}+\frac{\sigma^{2}}{2 \lambda^{2}}\right) \text { Freq }\left(\frac{x}{\sigma}-\frac{\sigma}{\lambda}\right)
\end{aligned}
$$

\section{A.3 Function for prompt enhanced sample}

$$
\begin{aligned}
& F_{\text {prompt }}\left(x, \sigma_{x} ; s, f_{+}, \lambda_{+}, f_{-}, \lambda_{-}\right) \\
= & \left(1-f_{+}-f_{-}\right) \frac{1}{\sqrt{2 \pi} \sigma_{x}} \exp \left(-\frac{x^{2}}{2 s^{2} \sigma_{x}^{2}}\right) \\
+ & f_{+} T\left(x, \sigma_{x} ; \lambda_{+}, s\right)+f_{-} T\left(-x, \sigma_{x} ; \lambda_{-}, s\right)
\end{aligned}
$$

where $T\left(x, \sigma_{x} ; \lambda, s\right)$ is same function defined in A.2, but takes $x$ and $\sigma_{x}$ as event-by-event input values. Fit parameters are $\lambda$ and resolution scale factor $s$.

$$
T\left(x, \sigma_{x} ; \lambda, s\right)=\frac{1}{\lambda} \exp \left(-\frac{x}{\lambda}+\frac{s^{2} \sigma_{x}^{2}}{2 \lambda^{2}}\right) \text { Freq }\left(\frac{x}{s \sigma_{x}}-\frac{s \sigma_{x}}{\lambda}\right)
$$




\section{Appendix B}

\section{Check of lifetime fitter using toy Monte Carlo samples}

The lifetime fitter is checked using toy Monte Carlo samples. According to decay exponential distributions including $\mathrm{K}$ factor and resolution smearing, toy Monte Carlo samples are created. We make 1000 trials in total. In each trial 10000 of $B^{-}$and 3000 of $\bar{B}^{0}$ events are generated. The resolution $\sigma_{c t^{*}}$ is generated to reproduce the data distribution. The resolution scale factor is set to be 1.5. Input lifetimes are $c \tau\left(B^{-}\right)=500 \mu \mathrm{m}, c \tau\left(\bar{B}^{0}\right)=460 \mu \mathrm{m}$. Sample compositions are set to $g_{-}=0.8$ for $D^{0}$ sample, and $g_{-}=0.15$ for $D^{*+}$ sample. Backgrounds are also generated in association with the $D^{0}$ and $D^{*+}$ signal, with the signal fractions being similar to the real muon dataset. In each trial toy Monte Carlo sample is fitted, and we look at the pull distributions of the fit results. Figure B.1 shows the pull distributions for $B^{-}$and $\bar{B}^{0}$ lifetimes, and their ratio. The mean fit uncertainties are 10.0 and $12.7 \mu \mathrm{m}$ for $B^{-}$and $\bar{B}^{0}$ lifetimes respectively, and 0.044 for the lifetime ratio. The pull distributions are centered at zero within uncertainty, and width is almost 1 . These results show that the fitter returns right answers. 

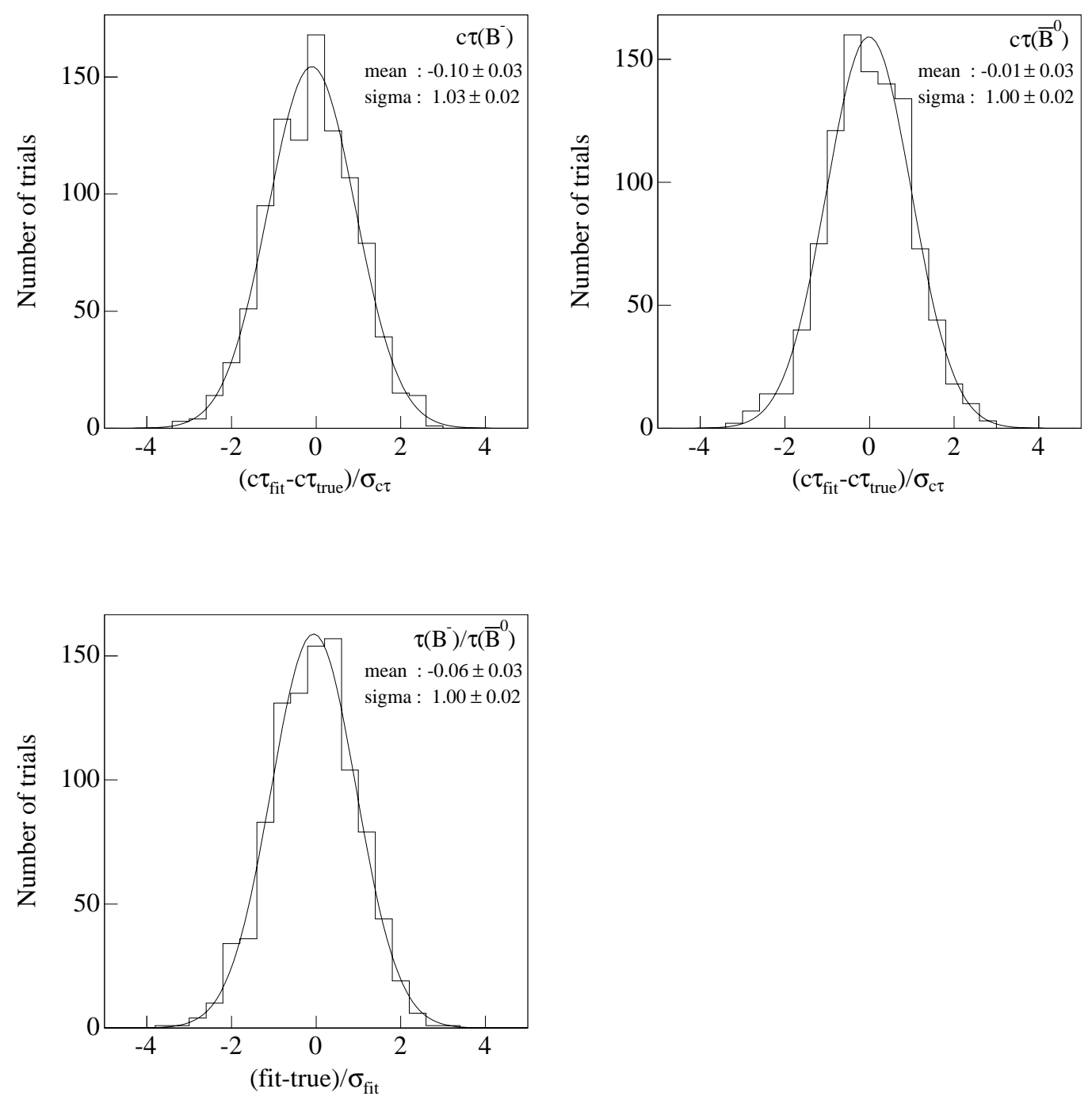

Figure B.1: Pull distributions to check lifetime fitter using toy Monte Carlo samples. 


\section{Appendix C}

\section{Semi-analytic form of $c t_{2}$ distribution for secondary charm mesons}

Suppose that a charm meson is produced by a decay of a $\bar{B}$ meson,

$$
\bar{B} \rightarrow D X,
$$

and that we measure the decay vertex of the charm meson and its decay length $L_{x y}$ with respect to the production point of the $\bar{B}$ meson, the primary vertex. The length $L_{x y}$ receives contributions from both the $B$ meson lifetime and the charm meson lifetime :

$$
\begin{aligned}
L_{x y} & =L_{B}+L_{D} \\
& =c t_{B}(\beta \gamma)_{B}+c t_{D}(\beta \gamma)_{D} .
\end{aligned}
$$

The variable $c t_{2}$ is defined by Eq. (5.2),

$$
c t_{2} \equiv L_{x y} \frac{m_{D}}{p_{T}^{D}}=L_{x y} /(\beta \gamma)_{D}
$$

and then we have

$$
\begin{aligned}
c t_{2} & =c t_{B} \frac{(\beta \gamma)_{B}}{(\beta \gamma)_{D}}+c t_{D} \\
& \equiv c t_{B} R+c t_{D}
\end{aligned}
$$

with

$$
R \equiv(\beta \gamma)_{B} /(\beta \gamma)_{D}
$$


If the $D$ meson is produced promptly, the first term vanishes and $c t_{2}$ reduces to a single exponential decay of the $D$ meson.

Now we want to express the functional form of the variable $c t_{2}$. The two terms both follow exponential functions. And the distribution of the variable $c t_{2}$ is given by the convolution of the two exponential functions. The integration can be performed analytically, and we find that, for a fixed $R$,

$$
\mathcal{F}\left(c t_{2}\right)=\frac{1}{c \tau_{B} R-c \tau_{D}}\left[\exp \left(-\frac{c t_{2}}{c \tau_{B} R}\right)-\exp \left(-\frac{c t_{2}}{c \tau_{D}}\right)\right] .
$$

In reality, the ratio $R$ has a finite spread, and we need to take the smearing into account,

$$
\mathcal{F}\left(c t_{2}\right)=\int d R \frac{H(R)}{c \tau_{B} R-c \tau_{D}}\left[\exp \left(-\frac{c t_{2}}{c \tau_{B} R}\right)-\exp \left(-\frac{c t_{2}}{c \tau_{D}}\right)\right],
$$

just as we do for the smearing with the momentum ratio $K$.

A typical distribution is shown in Figure C.1, along with one for promptly produced charm mesons.

However, an approximation is made when we derived these expressions. It is implicit in Eq. (C.1), which is valid when the opening angle between the directions of the $B$ and $D$ mesons is zero. It turns out that it is a pretty accurate approximation. However, we choose to use the distributions obtained using Monte Carlo events for completeness. 


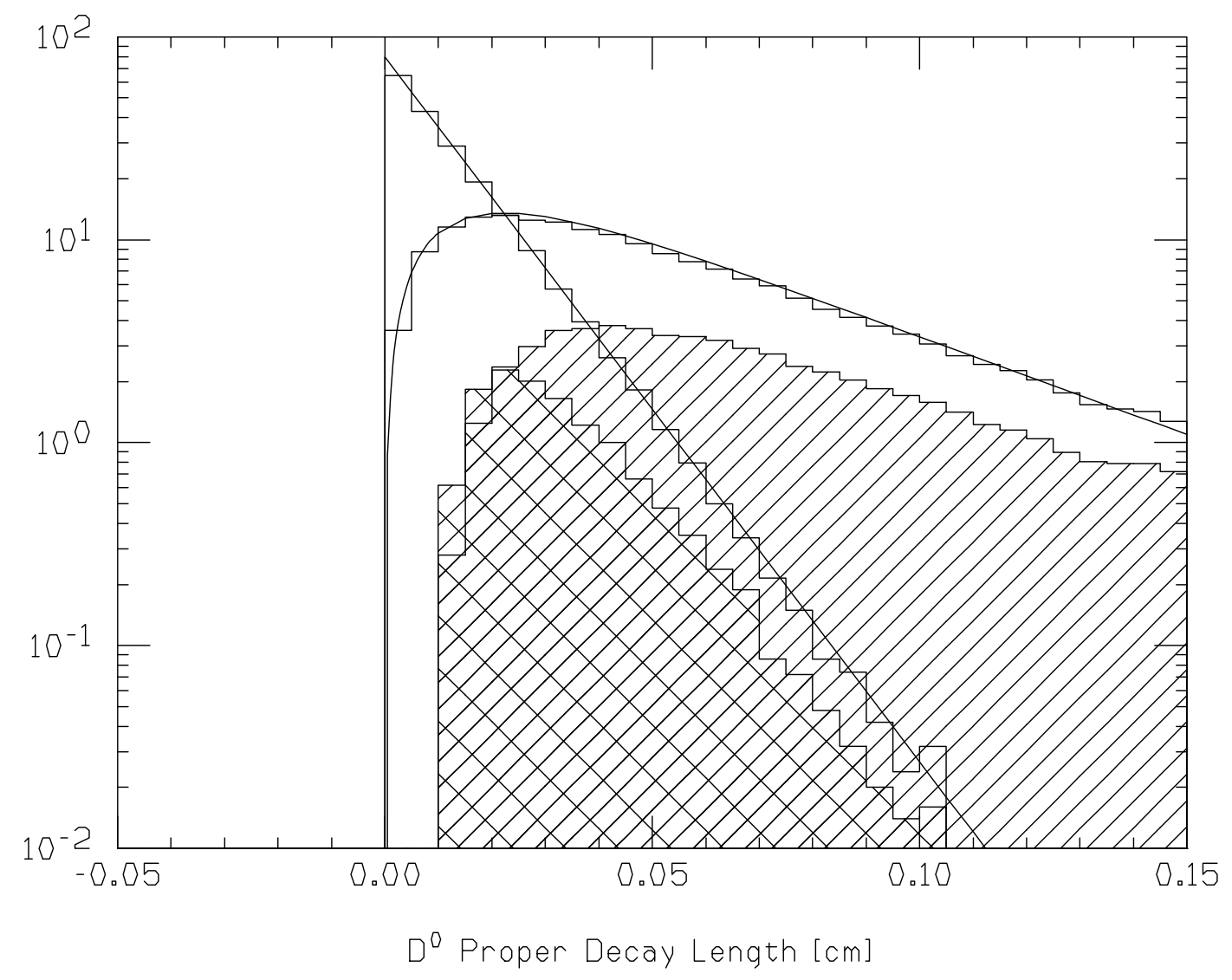

Figure C.1: Distributions of variable $c_{2}$ for prompt and secondary charm mesons (curves). Results of toy Monte Carlo experiments are overlaid as histograms. 



\section{Bibliography}

[1] Particle Data Group, Phys. Lett. B 592 (2004).

[2] M. Kobayashi and T. Maskawa, Prog. Theor. Phys., 49 (1973) 652.

[3] CDF public note 7649 (2005), http://www-cdf fnal.gov/physics/new/bottom/050330.blessed-bc-jpsimu/.

[4] CDF public note 7438 (2005), http://www-cdf.fnal.gov/physics/new/bottom/041118.blessed-bc-mass/.

[5] G. Buchalla, CERN-TH/2002018 (2002).

[6] I. I. Bigi et al., page 132 in "B decays" second edition, World Scientific, Singapore (1994).

[7] CDF Collaboration, F. Abe et al., Phys. Rev. D 58, 092002 (1998).

[8] CDF Collaboration, F. Abe et al., Phys. Rev. D 65, 092009 (2003).

[9] K. Abe et al., Phys. Rev. Lett. 79 (1997) 590.

[10] R. Barate et al., Phys. Lett. B 492 (2000) 275.

[11] D. Buskulic et al., Z, Ogts, C 71 (1996) 31.

[12] P. Abreu et al., Z. Phys. C 68 (1995) 13.

[13] W. Adam et al., Z. Phys. C 68 (1995) 363.

[14] J. Abdallah et al., Eur. Phys. J. C 33 (2004) 307.

[15] M. Acciari et al., Phys. Lett. B 438 (1998) 417.

[16] R. Akers et al., Z. Phys. C. 67 (1995) 379.

[17] G. Abbiendi et al., Eur. Phys. J. C 12 (2000) 609.

[18] B. Aubert et al., Phys. Rev. Lett. 87 (2001) 201803. 
[19] B. Aubert et al., Phys. Rev. Lett. 89 (2002) 011802, erratumibid. 89 (2002) 169903.

[20] B. Aubert et al., Phys. Rev. D 67 (2003) 072002.

[21] B. Aubert et al., Phys. Rev. D 67 (2003) 091101.

[22] B. Aubert et al., hep-ex/0408039 (2004).

[23] K. Abe et al., Phys. Rev. D 71 (2005) 072003.

[24] CDF public note 7409 (2004), http://www-cdf.fnal.gov/physics/new/bottom/040428.blessed-lft2/.

[25] CDF public note 7514 (2005) (analysis performed in this thesis), http://www-cdf .fnal.gov/physics/new/bottom/050224.blessed-bsemi-life/.

[26] CDF public note 7386 (2005), http://www-cdf.fnal.gov/physics/new/bottom/050303.blessed-bhadlife/.

[27] V. M. Abazov et al., Phys. Rev. Lett. 94 (2005) 042001.

[28] V. M. Abazov et al., Phys. Rev. Lett. 94 (2005) 102001.

[29] V. M. Abazov et al., hep-ex/0410052 (2004).

[30] D. Buskulic et al., Phys. Lett. B 377 (1996) 205.

[31] D. Buskulic et al., Eur. Phys. J. C 4 (1998) 367.

[32] P. abreu et al., Eur. Phys. J. C 16 (2000) 555.

[33] P. abreu et al., Eur. Phys. J. C 18 (2000) 229.

[34] K. Ackerstaff et al., Phys. Lett. B 426 (1998) 161.

[35] K. Ackerstaff et al., Eur. Phys. J. C 2 (1998) 407.

[36] F. Abe et al., Phys. Rev. D 59 (1999) 032004.

[37] F. Abe et al., Phys. Rev. D 57 (1998) 5382.

[38] V. M. Abazov et al., D $\emptyset$ note 4729-CONF (2005).

[39] Heavy Flavor Averaging Group, hep-ex/0505100 (2005).

[40] K. Anikeev et al., hep-ph/0201071 (2002). 
[41] E. J. N. Wilson, "An Introduction to Particle Accelerators", Oxford University Press (2001).

[42] Tevatron Run II Handbook, http://www-bd.fnal.gov/lug/runII_handbook/.

[43] V. Shiltsev, FERMILAB-Conf-04/123-AD (2004).

[44] The CDF II Collaboration, FERMILAB-Pub-96/390-E (1996).

[45] The CDF Collaboration, Nucl. Instr. Meth., A271 (1988) 387

[46] M. Sciveres et al., Nucl. Instr. Meth., A435 (1999) 58.

[47] A. Sill et al., Nucl. Instr. Meth., A447 (2000) 1.

[48] R. Blair et al., CDF internal note 760.

[49] T. Kamon et al., Nucl. Instr. Meth., A213 (1983) 261.

[50] T. Balka et al., Nucl. Instr. Meth., A267 (1988) 272.

[51] G. Bauer et al., CDF internal note 4623 (1998).

[52] A. Mukherjee, CDF internal note 5490 (1995).

[53] P. Gatti, CDF internal note 5561 (2000).

[54] S. Menzemer, CDF internal note 5968 (2003).

[55] M. Gold, CDF internal note 913 (1989).

[56] J. Marriner, CDF internal note 1996 (1993).

[57] K. Anikeev et al., CDF internal note 5092 (1999).

[58] CDF internal note 2724 (1999), and http://www. lns . cornell.edu/public/CLEO/soft/QQ/.

[59] D. Lange et al., Nucl. Instr. Meth., A462 (2001) 152.

[60] "CDF Detector Simulation", http://www-cdf.fnal.gov/cdfsim/cdfsim_main.html.

[61] D.Acosta et al., FERMILAB-Pub-040440-E (2004).

[62] N. Isgur, D. Scora, B. Grinstein, and M. B. Wise, Phys. Rev. D 39799 (1989) . 
[63] P. Nason, S. Dawson and R. K. Ellis, Nucl. Phys. B 327 (1989) 49.

[64] D. Scora and N.Isgur, Phys. Rev. D 522783 (1995).

[65] C. Chen et al., CDF internal note 6314 (2003).

[66] CDF Bs Mixing Working Group, CDF internal note 7500 (2005).

[67] T. Sjostrand et al., hep-ph/0108264 (2001).

[68] F. Ukegawa, A. B. Wicklund, CDF internal note 1516 (1991).

[69] D. Acosta et al., Phys. Rev. Lett. 94101803 (2005).

[70] K. Hartkorn, H.-G. Moser, Eur. Phys. J. C 8, 381-383 (1999).

[71] F. Ukegawa, CDF internal note 7756 (2005). 